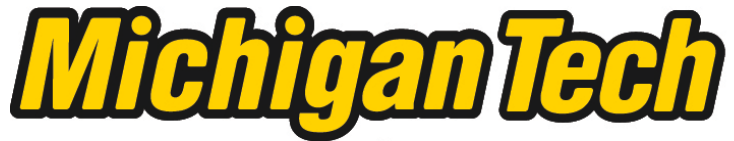 \\ Michigan Technological University Create the Future Digital Commons @ Michigan Tech
}

Dissertations, Master's Theses and Master's Reports - Open

Dissertations, Master's Theses and Master's

Reports

2014

\section{QUANTUM CORRELATIONS OF LIGHTS IN MACROSCOPIC ENVIRONMENTS}

Yong Meng Sua

Michigan Technological University

Follow this and additional works at: https://digitalcommons.mtu.edu/etds

Part of the Optics Commons, and the Quantum Physics Commons Copyright 2014 Yong Meng Sua

\section{Recommended Citation}

Sua, Yong Meng, "QUANTUM CORRELATIONS OF LIGHTS IN MACROSCOPIC ENVIRONMENTS", Dissertation, Michigan Technological University, 2014.

https://doi.org/10.37099/mtu.dc.etds/769

Follow this and additional works at: https://digitalcommons.mtu.edu/etds

Part of the Optics Commons, and the Quantum Physics Commons 


\title{
QUANTUM CORRELATIONS OF LIGHTS IN MACROSCOPIC ENVIRONMENTS
}

\author{
By
}

Yong Meng Sua

\begin{abstract}
A DISSERTATION
Submitted in partial fulfillment of the requirements for the degree of DOCTOR OF PHILOSOPHY

In Engineering Physics
\end{abstract}

MICHIGAN TECHNOLOGICAL UNIVERSITY

2014

C 2014 Yong Meng Sua 

This dissertation has been approved in partial fulfillment of the requirements for the Degree of DOCTOR OF PHILOSOPHY in Engineering Physics

Department of Physics

Dissertation Co-Advisor: $\quad$ Dr. Kim Fook Lee

Dissertation Co-Advisor: Dr. Jacek Borysow

Committee Member: $\quad$ Dr. Miguel Levy

Committee Member: Dr. Ranjit Pati

Committee Member: Dr. Durdu Gurney

Department Chair: Dr. Ravindra Pandey 

To My Beloved Parents 



\section{Table of Contents}

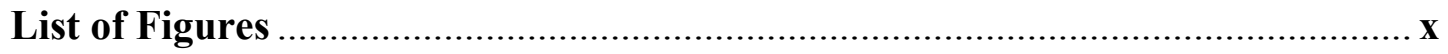

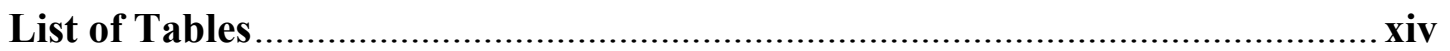

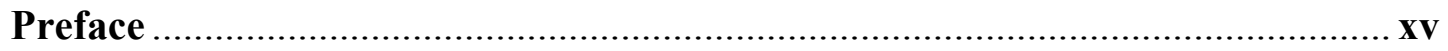

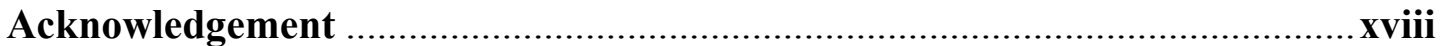

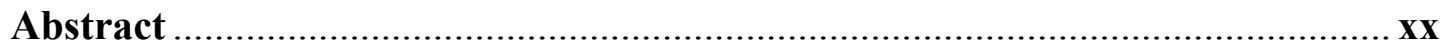

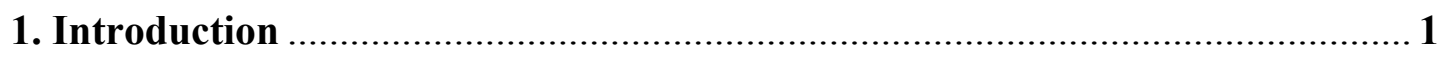

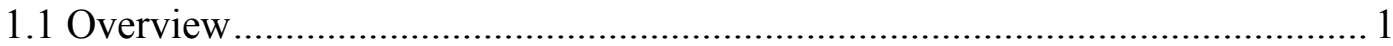

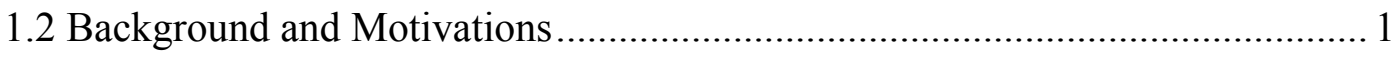

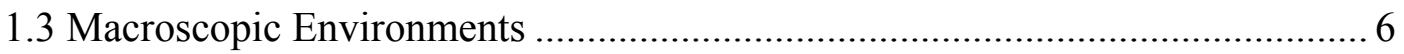

1.4 Quantum Superposition and Entanglement ..................................................... 7

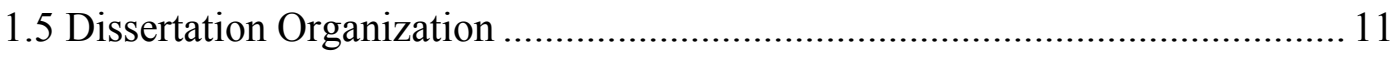

2. Macroscopic Mechanical Correlations of Two Mirrors ……………….............13

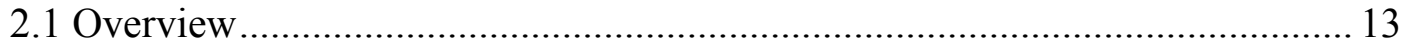

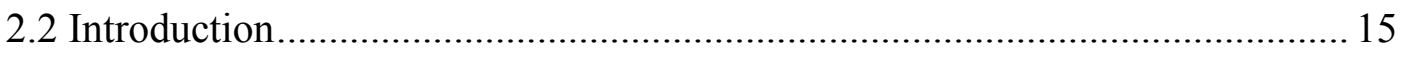

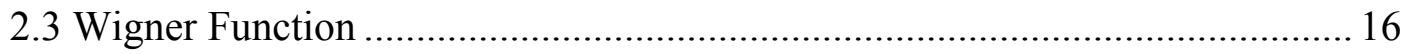

2.4 Single Photons Spatial Compass State............................................................. 19

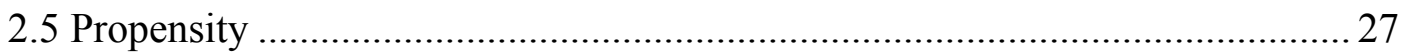

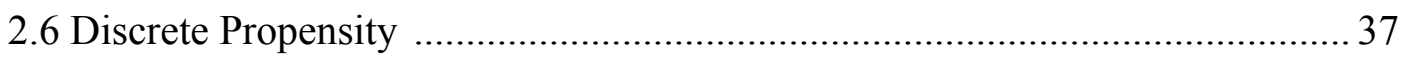

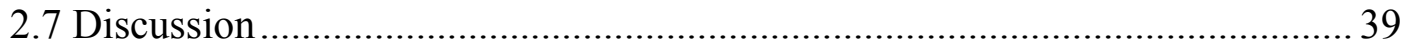

3. Intrinsic Quantum Correlation of Weak Coherent States ................................ 43

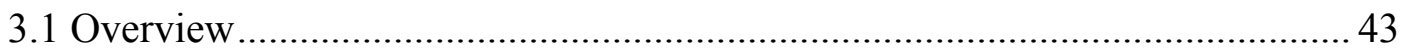

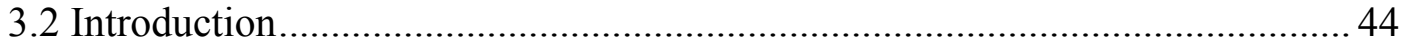

3.3 Weak Coherent States Bipartite Correlations ................................................. 46

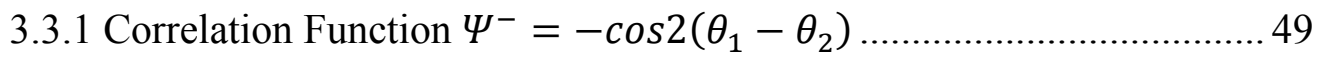

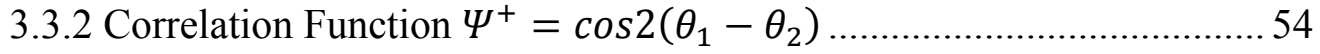

3.3.1 Correlation Function $\phi^{-}=-\cos 2\left(\theta_{1}+\theta_{2}\right) \ldots \ldots \ldots \ldots \ldots \ldots \ldots \ldots \ldots \ldots \ldots \ldots \ldots . . . . .56$

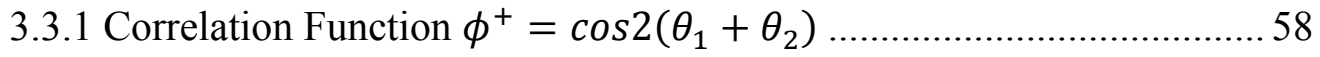

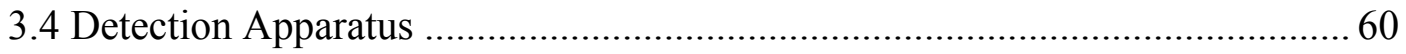


3.4.1 Photodetectors and Transimpedance Amplifier ................................6 60

3.4.2 Oscilloscope and Spectrum Analyzer.............................................. 62

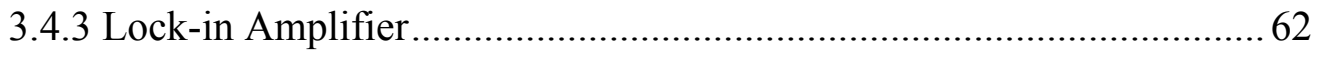

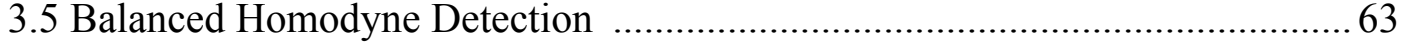

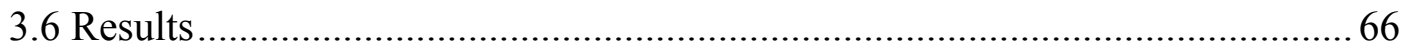

3.6.1 Weak Coherent States Interference and Correlations.......................... 66

3.6.2 Weak Coherent States Bipartite Correlations (CSBC) ........................ 70

3.6.3 Bit correlations measurement ........................................................ 72

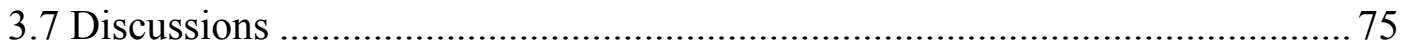

4. Intrinsic Quantum Correlation of Weak Coherent States............................. 79

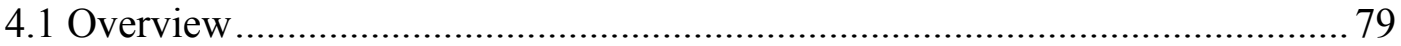

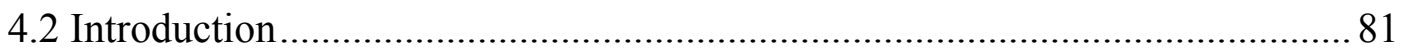

4.2.1 Heralded Single Photon Source ...................................................... 81

4.2.2 Entangled Photon Source.............................................................. 82

4.2.3 Fiber based Correlated and Entangled Photon Source ........................ 83

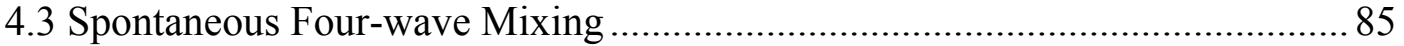

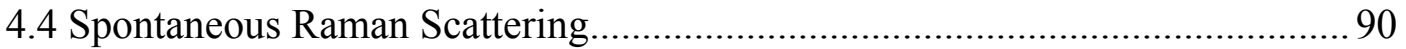

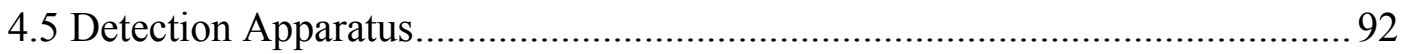

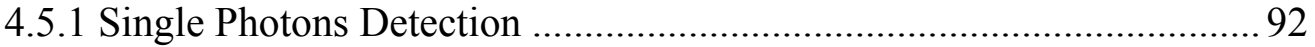

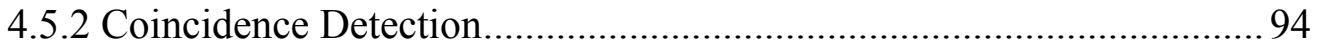

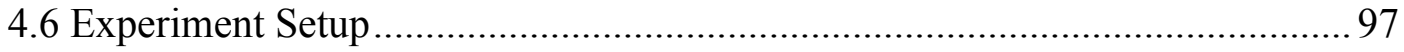

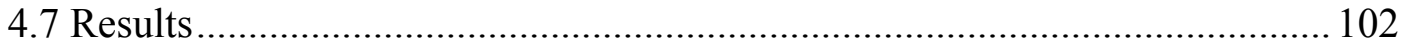

4.7.1 Single Photons Count ...................................................................... 102

4.7.2 Characterization of Correlated Photon Source ................................... 105

4.7.3 Characterization of Entangled Photon Source................................... 109

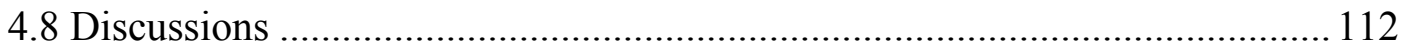

5. Photon pairs Propagate Through Multiple Scattering Media...................... 117

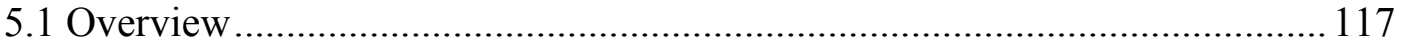

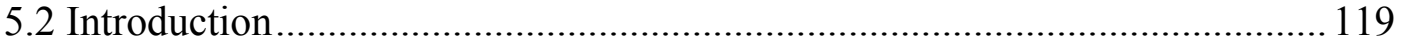


5.3 Evolution of Annihilation Operator ..................................................... 121

5.4 Joint Probability of Two-photon Detection ................................................. 125

5.4.1 Polarization-correlated Photon Pair.................................................... 125

5.4.2 Polarization-correlated Photon Pair................................................. 127

5.5 Multiple Scattering Random Media............................................................. 129

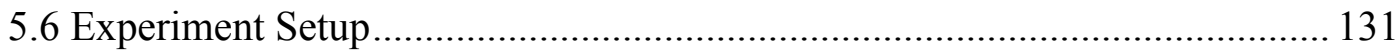

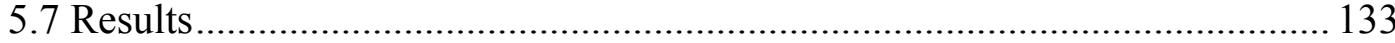

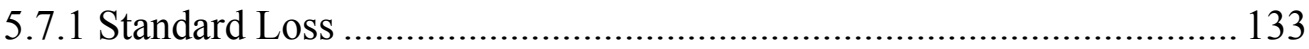

5.7.2 Multiple Scattering Random Media ............................................. 137

5.7.3 Influence of Raman photons in Fiber Source ................................... 141

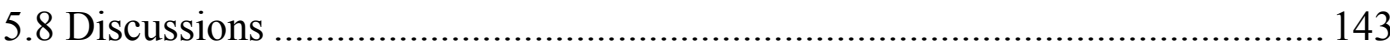

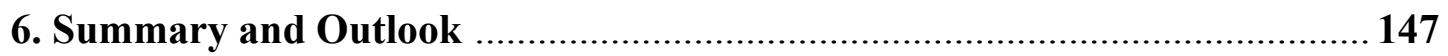

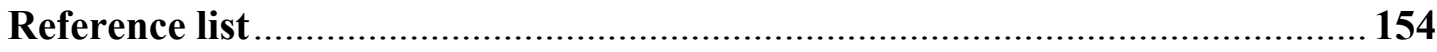

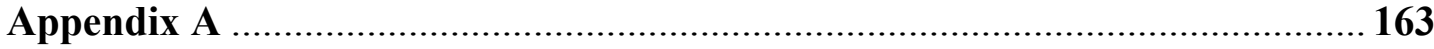

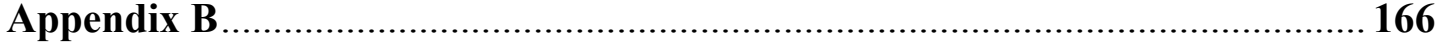

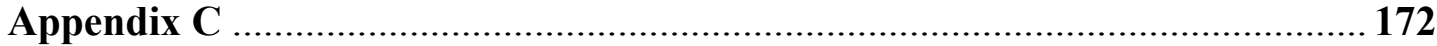

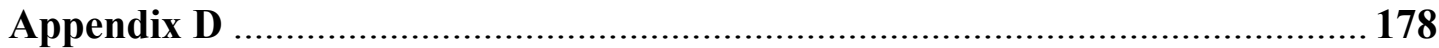

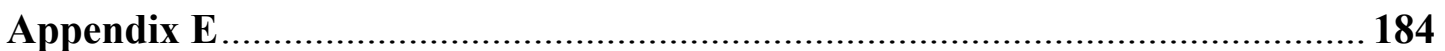

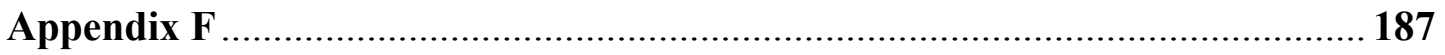

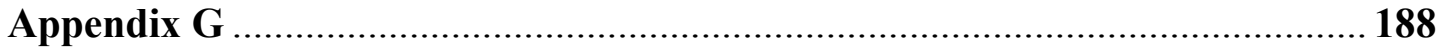




\section{List of Figures}

Figure 2.1. Gaussian Wigner function: (Left) 2 dimensional contour plot; (Right) 3dimensional contour plot.

Figure 2.2. The proposed experimental setup for measuring the propensity of two spatial compass states and spatial correlations of two mirrors. ( $\mathrm{SMF}=$ single mode fiber, $\mathrm{BS}=$ beam splitter, $\mathrm{L}=$ lense, $\mathrm{M}=$ mirror, $\mathrm{SPD}=$ single photon detector) 20

Figure 2.3. The phase space plots of a spatial compass state with realistic parameters: $\sigma_{a}=100 \mu \mathrm{m}, \mathrm{a}=1.0 \mathrm{~cm}, \mathrm{f}=5.0 \mathrm{~cm}$, and $\lambda=632 \mathrm{~nm} . \mathcal{W}_{1,2}(x, p)^{t t}$, (b) $\mathcal{W}_{1,2}(x, p)^{r r}$ 25

Figure 2.4. The phase space plots of a spatial compass state with realistic parameters: $\sigma_{a}=100 \mu \mathrm{m}, \mathrm{a}=1.0 \mathrm{~cm}, \mathrm{f}=5.0 \mathrm{~cm}$, and $\lambda=632 \mathrm{~nm}$. (a) 2 dimension plot of chessboard pattern, (b) 3 dimension plot of chessboard pattern .. 26

Figure 2.5. Phase space distribution of the four corner fringes between the two lumps of $\mathcal{W}(x, p)_{t t}$, and $\mathcal{W}(x, p)_{r r}$

Figure 2.6. The geometrical dynamic picture of the imaging system for achieving the EPR position correlations in measuring the propensity. 30

Figure 2.7. The geometrical dynamic picture of the imaging system for achieving the EPR momentum correlations in measuring the propensity

Figure 2.8. (a) The 2D (b) 3D chessboard patterns of the propensity in position $d_{x}$ and momentum $d_{p}$ (c) The 3D plots of the center spot. 34

Figure 2.9. (a) $3 \mathrm{D}$ propensity plots of the random-numbers position $d_{x}$ and momentum $d_{p}$. (b) Side view of random-numbers propensity plots .......35

Figure 2.10. The plot of $(\Delta X)^{2}(\Delta P)^{2}$ as a function of $a$ and $D_{p}$, showing the criterion for the EPR entanglement in the region $<1$

Figure 2.11. Chessboard pattern of discretized propensity $\mathcal{P}_{b}(m, n) \sim \mid \cos (m \pi)+$ $\left.\cos (n \pi)\right|^{2}$ in random number $m$ and $n$

Figure 3.1. Experiment setup for demonstration of Correlation function $\Psi^{-}=$ $-\cos 2\left(\theta_{1}-\theta_{2}\right)$ with weak coherent states. BS (beam splitter); PBS (polarization beam splitter); HWP and QWP (half- and quarter-wave plates); D (photodiode)

Figure 3.2. Experiment setup used for demonstration of Correlation function $\Psi^{+}=$ $\cos 2\left(\theta_{1}-\theta_{2}\right)$

Figure 3.3. Experiment setup used for demonstration of Correlation function $\phi^{+}=$ $\cos 2\left(\theta_{1}-\theta_{2}\right)$

Figure 3.4. Experiment setup used for the demonstration of Correlation function $\phi^{-}=\cos 2\left(\theta_{1}-\theta_{2}\right)$

Figure 3.5. Schematic diagram of balanced detection and transimpedance amplifier circuit

Figure 3.6. Balanced Homodyne detection. 
Figure 3.7. Frequency spectrum of balanced homodyne detector indicating the electronics noise level without any light and the shot noise level with the presence of the local oscillator field

Figure 3.8. (a) Shot noise (blue solid line) of the weak LO field (1 mW) plus coherent state $(0.03 \mathrm{~mW})$ and electronic noise level (red dots); the corresponding beat signals for $\theta_{1}=\theta_{2}$ at detector $\mathrm{A}(\mathrm{b})$ and detector $\mathrm{B}$ (c), and their multiplied signal (d)

Figure 3.9. (a) Shot noises (blue solid line) of the weak LO field $(0.001 \mathrm{~mW})$ plus weak coherent state $(0.001 \mathrm{~mW})$ and electronic noise level (red dots); the corresponding beat signals for $\theta_{1}=\theta_{2}$ at detector A (b) and detector B (c), and their multiplied signal (d)

Figure 3.10. The experimental observation of the correlation functions i) $\Psi^{-}=$ $-\cos 2\left(\theta_{1}-\theta_{2}\right)$ for the case $\theta_{1}=\theta_{2}$. The observed random noise beat signal in (a) detector $A$, (b) detector $B$. (c) Multiplied beat signals. ii) $\Psi^{-}=\cos 2\left(\theta_{1}-\theta_{2}\right)$ for the case $\theta_{1}=\theta_{2}$. The observed random noise beat signal in (d) detector A, (e) detector B, (f) Multiplied beat signals. 69

Figure 3.11. Experimental measurement of Bi-partite correlation functions (a) $-\cos 2\left(\theta_{1}-\theta_{2}\right),(b)-\cos 2\left(\theta_{1}+\theta_{2}\right),(\mathrm{c}) \cos 2\left(\theta_{1}-\theta_{2}\right),(d) \cos 2\left(\theta_{1}+\right.$ $\left.\theta_{2}\right)$ 71

Figure 3.12. a) Single period of interference signal measured at observer A (red solid line) compared to b) piezoelectric driving voltage (blue dashed line), which is used as reference phase in the lock-in amplifier 72

Figure 3.13. Experiment setup for demonstration of the bit generation and

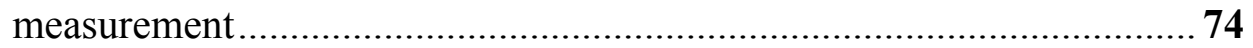

Figure 3.14. Bit correlation of two weak light fields a) $-\cos 2\left(\theta_{1}-\theta_{2}\right)$, (b) $-\cos 2\left(\theta_{1}+\theta_{2}\right)$, (c) $\cos 2\left(\theta_{1}-\theta_{2}\right)$, (d) $\cos 2\left(\theta_{1}+\theta_{2}\right) \ldots \ldots \ldots \ldots \ldots . . .74$

Figure 4.1. Illustration of degenerate Spontaneous four wave mixing process where two photons at $\omega_{1}$ are annihilated and two photons at $\omega_{3}$ and $\omega_{4}$ are created....

Figure 4.2. Energy level diagram of Stokes and anti-Stokes spontaneous Raman scattering 90

Figure 4.3. Illustration of electrical gate pulses precisely coincides with incoming single photon pulses for the single photon detection.....

Figure 4.4. Plot of quantum efficiency versus the dark count probability of single photon detector at different biased voltages above the breakdown voltage.

Figure 4.5. A typical histogram acquired by the multichannel scaler. The time bin with highest counts is the Coincidence count, and the rest of the bins are each accidental coincidence count. 95

Figure 4.6. Diagram shows timing correlation of coincidence count and accidentalcoincidence count from the pulse sequences in APD1 for idler photon and APD2 for signal photon. 96 
Figure 4.7. Schematic Layout of single photon detection and coincidence detection system

Figure 4.8. Layout of experiment setup. FC (fiber-to-free space collimators); PBS (polarization beam splitter); HWP and QWP (half- and quarter-wave plates); DWDM (dense wavelength division multiplexer); APD (Avalanche photodiode) ........................................................................100

Figure 4.9. Transmission spectrum of cascaded DWDM filters for signal (1547.4 nm) and idler channel $(1560.6 \mathrm{~nm})$..... 101

Figure 4.10. A graph showing single photon counts per pulse of Signal (Blue dot) and Idler (Black square) photon as a function of number of pump photon per pulse when the HNLF is in room temperature $(300 \mathrm{~K})$ 103

Figure 4.11. A graph showing single photon counts (per pulse) of Signal (Blue dot) and Idler (Black square) photon as a function of number of pump photon per pulse when the HNLF is cooled to $77 \mathrm{~K}$ by immersing in liquid nitrogen 104

Figure 4.12. A plot of total coincidence counts (Blue dot) and accidental coincidence counts (Red diamond) as a function of number of pump photon per pulse with the HNLF in room temperature $(300 \mathrm{~K})$ 106

Figure 4.13. A plot of total coincidence counts (Blue dot) and accidental coincidence counts (Red diamond) as a function of number of pump photon per pulse with the HNLF in $77 \mathrm{~K}$ 106

Figure 4.14. Coincidence to accidental coincidence ratio (CAR) versus number of pump photon per pulse with HNLF at $300 \mathrm{~K}$ (Blue dot) and $77 \mathrm{~K}$ (Red triangle)

108

Figure 4.15. Two-photon interference fringes with HNLF at $300 \mathrm{~K}$ with (i) $\theta_{1}=0^{\circ}$ (Blue dot) and (ii) $\theta_{1}=-45^{\circ}$ (Red dot). The solid lines are the theoretical curve fitting..

110

Figure 4.16. Two-photon interference fringes with HNLF at $77 \mathrm{~K}$ (i) $\theta_{1}=0^{\circ}$ (Blue dot) and (ii) $\theta_{1}=-45^{\circ}$ (Red dot). The solid lines are the theoretical curve fitting

Figure 5.1. A simple model for one photon of polarization-correlated/-entangled photon-pair propagating through a random medium, where annihilation operator $\hat{a}_{i}$ of the idler photon goes through coherent $\left(\hat{\mathrm{a}}_{\mathrm{o}}\right)$ and incoherent $(\hat{c})$ part of the scattering process in random medium ..........122

Figure 5.2. Experiment setup for measuring CAR and two-photon interference of the signal photon in a normal channel and the idler photon experiencing multiple scattering events. FC (fiber-to-free space collimators); PBS (polarization beam splitter); HWP and QWP (half- and quarter-wave plates); DWDM (dense wavelength division multiplexer); APD (Avalanche photodiode) 132

Figure 5.3. The Coincidence to accidental coincidence ratio (CAR) versus pump power with different attenuations. (Green square $=1 \mathrm{~dB})$, (Blue diamond $=3 \mathrm{~dB})$ and $($ Red dot $=5 \mathrm{~dB})$. 
Figure 5.4. The measured CAR estimates visibility (Blue box) and maximum CAR (Solid circle) as a function of standard loss....

Figure 5.5. Two-photon interference fringes as a function of analyzer relative angle for the standard loss of $5 \mathrm{~dB}$ with HNLF at $300 \mathrm{~K}$. (Blue dot) and (ii) $\theta_{1}=$ $-45^{\circ}$ (Red dot). The solid lines are the theoretical curve fitting

Figure 5.6. The measured two-photon interference visibility $\mathcal{V}_{\text {ent }}$ (Red box) and predicted visibility $\mathcal{V}_{\text {cor }}$ (Solid circle) versus standard losses.....

Figure 5.7. The Coincidence to accidental coincidence ratio (CAR) of correlated photon pair versus pump power for different scattering mean free path. (Black circle, $\ell=0.010 \mathrm{~m})($ Green diamond, $\ell=0.010 \mathrm{~m}$ ), (Blue dot $\ell$ $=0.004 \mathrm{~m})$ and $(\operatorname{Red}$ box $\ell=0.004 \mathrm{~m})$ 138

Figure 5.8. The predicted visibility for correlated photon pair, $\mathcal{V}_{c o r}$ as a function of scattering mean free path 139

Figure 5.9. The $\mathcal{V}_{\text {ent }}($ Red dot) versus scattering mean free path 140

Figure 5.10. Two-photon interference fringes (Blue square) as a function of relative polarization angle, the dotted red line are curve fitting. Coincidence counts was accumulated for 68 seconds 140

Figure 5.11. The $\mathcal{V}_{\text {ent }}$ (Blue square) and $\mathcal{V}_{\text {cor }}($ Red dot) versus mean free path, the solid lines are fitting curves for $\mathcal{V}_{\text {ent }}$ and $\mathcal{V}_{\text {cor }}$. The dashed line is the visibility measured with $3 \mathrm{~dB}$ standard loss. 


\section{List of Tables}

Table 4.1 Violation of Bell's inequality for entangled state $\left|H_{i} V_{s}\right\rangle-\left|V_{i} H_{s}\right\rangle \ldots \ldots \ldots \ldots \ldots \ldots \ldots \ldots . . . . . . . .112$

Table 5.1 Properties of the random medium samples..........................................131

Table 5.2 Summary of the results for the scattering random media .......................146 


\section{Preface}

This dissertation is based on my $\mathrm{PhD}$ (Engineering Physics) research work conducted at Depament of Physics, Michigan Technological University during the period August 2009-December 2013. This preface explains my contribution in the work of each chapter that makes up the body of this Dissertation. This Dissertation includes texts, figures and data published in the following 3 peer-reviewed journal articles and another article which is currently in peer-review process.

1. Yong Meng Sua, Erin Scanlon, Travis Beaulieu, Viktor Bollen, and Kim Fook Lee, "Intrinsic quantum correlations of weak coherent states for quantum communication," Phys. Rev. A 83, 030302(R) (2011).

2. Yong Meng Sua, and Kim Fook Lee, "Macroscopic mechanical correlations using single-photon spatial compass state and operational Wigner function," Phys. Rev. A 85, 062113 (2012).

3. Yong Meng Sua, John Malowicki, Masaaki Hirano, and Kim Fook Lee, "Generation of high purity entangled photon-pair in a short highly nonlinear fiber," Optics Letters, 38, 73-75 (2013).

4. Yong Meng Sua, John Malowicki, and Kim Fook Lee, "Quantum Correlation and Interference of Fiber based Telecom-band Photon-Pairs in Multiple Scattering Media," (Under review) 
I (Yong Meng Sua) have coauthored all the articles listed above I have obtained the necessary copyright permission from publishers (see Appendix G) to reproduce text, figures and data from the above listed journal articles in the present dissertation titled:

"QUANTUM CORRELATIONS OF LIGHTS IN MACROSCOPIC ENVIRONMENTS"

In the first publication in "Physical Review A" (Chapter 3), we demonstrated bipartite correlations of two weak coherent states over a distance of $10 \mathrm{~km}$ which can be used as a supplemental resource to the existence QKD protocols such as coherent state DPS-QKD and decoy state BB84 protocol. I designed and demonstrated this experiment in our laboratory. The data analysis was carried out by me and my advisor, Dr. Kim Fook Lee. Three former undergraduate students from department of Physics which are Erin Scanlon, Travis Beaulieu and Viktor Bollen contributed in data acquisition and instrumentation.

In the second article published in "Physical Review A" (Chapter 2), we proposed a measurement scheme for observing quantum correlations and entanglement in the spatial properties of two macroscopic mirrors. We showed that realistic motions of measuring the propensity in the imaging system can extract the Einstein-Podolsky-Rosen correlations of two mirrors. I carried out the mathematical derivation and calculation. Dr. Kim Fook Lee and me analyzed the calculation results and proposed the measurement scheme.

In the third work published in "Optics Letter" (Chapter 4), we generated correlated and entangled photon pairs at telecom wavelengths through four-wave mixing in a $10 \mathrm{~m}$ highly nonlinear fiber. The $10 \mathrm{~m}$ highly nonlinear fiber was xvi 
fabricated and characterized by our collaborator Mr. Massaki Hirano from Sumitomo Electric, Japan. I designed and conducted the experiment. I designed and built single photon detection and coincidence detection system. The data analysis was carried out together by me and Dr. Kim Fook Lee with technical input of Mr. John Malowicki from Air Force research Laboratory.

In the last work (Chapter 5) which is currently in peer-review process, we demonstrated that both standard losses and multiple scattering in transmission channel are detrimental to the quantum correlation of photon-pair. We also found that Raman noise photons in fiber source will enhance the depolarization effect in multiple scattering. This is extension of our work published in "Optics Letter", addressing the issue in long distance quantum key distribution. I designed and performed the experiment with helpful discussion from Mr John Malowicki. Data analysis was carried out together by me and Dr. Kim Fook Lee. Subsequently, we proposed a model to explain our discoveries in this work.

Chapter 1 is the introduction to this Dissertation; Chapter 6 summarizes the important findings of this Dissertation and provides suggestions for the future work. 


\section{Acknowledgements}

Over the past five years, there are many people who I owe thanks for their help and support. First and foremost, I owe a great deal to my advisor, Dr. Kim Fook Lee, for his outstanding guidance and support. He taught me virtually all of my optics skills and shown me the beauty of quantum optics. I also had the privilege of working with Dr. Jacek Borysow, who helped with the final phases of my graduate study and provided invaluable advices for my dissertation and oral defense. I'm thankful for his great mentorship and for teaching me the beautiful Raman spectroscopy experiment. I'm also grateful to have Dr. Miguel Levy, Dr. Ranjit Pati and Dr. Durdu Guney as my advisory committee member.

Over the past years I have had many favors from other research groups in Physics Department. I'm thankful to Dr. Miguel Levy who lent me the tunable laser source and many other optical components. I'm also grateful to Dr. Claudio Mazzoleni who lent me so many optical and electronic instruments. Not to forget Dr. Kelken Chang, who borrowed us the delay generator from the Cloud Physics Laboratory, I would not be able to detect a photon pair without it.

I'm particularly indebted to Department Chair of Physics, Dr. Ravindra Pandey who is ever supportive to all Physics graduate students. I appreciate the assistance and support from all superb staffs in Physics Department throughout my graduate career as well.

I had the pleasure to work with many highly motivated and bright students, from all of whom I have benefited a lot. Viktor Bollen has been very helpful in 
instrumentation for our lab and never hesitates to share his expertise in electronics and Labview programming with me. I'm thankful to Paul Rojas for his effort in the developing the single photon detector. I'm also enjoying working with Rachel Blaser, Erin Scanlon and Travis Beaulieu in our lab in lovely summer of 2010. I wish them well in their career.

In addition, I'm fortunate to have genuine friendship from the fellow graduate students in Physics Department. Especially, I will always remember many interesting and inspiring conversations with Kamal Dhungana, Boyi Hao, and Abhilash Kantamneni about physics and everything else. I wish them well in their graduate study and future career. May the Force be with you!

Most of all, I want to thank my loving parents, brother and sisters, who have always encouraged me throughout my graduate career. This thesis is dedicated to them. 


\begin{abstract}
This dissertation presents a detailed study in exploring quantum correlations of lights in macroscopic environments. We have explored quantum correlations of single photons, weak coherent states, and polarization-correlated/polarizationentangled photons in macroscopic environments. These included macroscopic mirrors, macroscopic photon number, spatially separated observers, noisy photons source and propagation medium with loss or disturbances.
\end{abstract}

We proposed a measurement scheme for observing quantum correlations and entanglement in the spatial properties of two macroscopic mirrors using single photons spatial compass state. We explored the phase space distribution features of spatial compass states, such as chessboard pattern by using the Wigner function. The displacement and tilt correlations of the two mirrors were manifested through the propensities of the compass states. This technique can be used to extract EinsteinPodolsky-Rosen correlations (EPR) of the two mirrors. We then formulated the discrete-like property of the propensity $\mathcal{P}_{b}(m, n)$, which can be used to explore environmental perturbed quantum jumps of the EPR correlations in phase space. With single photons spatial compass state, the variances in position and momentum are much smaller than standard quantum limit when using a Gaussian TEMo0 beam.

We observed intrinsic quantum correlations of weak coherent states between two parties through balanced homodyne detection. Our scheme can be used as a supplement to decoy-state BB84 protocol and differential phase-shift QKD protocol. We prepared four types of bipartite correlations $\pm \cos 2\left(\theta_{1} \pm \theta_{2}\right)$ that shared between 
two parties. We also demonstrated bits correlations between two parties separated by $10 \mathrm{~km}$ optical fiber. The bits information will be protected by the large quantum phase fluctuation of weak coherent states, adding another physical layer of security to these protocols for quantum key distribution.

Using $10 \mathrm{~m}$ of highly nonlinear fiber (HNLF) at $77 \mathrm{~K}$, we observed coincidence to accidental-coincidence ratio of $130 \pm 5$ for correlated photon-pair and Two-Photon Interference visibility $>98 \%$ entangled photon-pair. We also verified the non-local behavior of polarization-entangled photon pair by violating Clauser-HorneShimony-Holt Bell's inequality by more than 12 standard deviations. With the HNLF at $300 \mathrm{~K}(77 \mathrm{~K})$, photon-pair production rate about factor 3(2) higher than a $300 \mathrm{~m}$ dispersion-shifted fiber is observed. Then, we studied quantum correlation and interference of photon-pairs; with one photon of the photon-pair experiencing multiple scattering in a random medium. We observed that depolarization noise photon in multiple scattering degrading the purity of photon-pair, and the existence of Raman noise photon in a photon-pair source will contribute to the depolarization affect. We found that quantum correlation of polarization-entangled photon-pair is better preserved than polarization-correlated photon-pair as one photon of the photon-pair scattered through a random medium. Our findings showed that high purity polarization-entangled photon-pair is better candidate for long distance quantum key distribution. 



\section{Chapter 1}

\section{Introduction}

\subsection{Overview}

First and foremost, I shall embark on my dissertation by a brief introduction on Quantum Information Science and describe the motivations of this work. In following section, I will discuss on the definition of the macroscopic environments from history point of view and how we define it in our work. Then, I will proceed by introducing the fundamental features of quantum mechanics, which are quantum superposition and entanglement. The nonlocal behavior of the entanglement was once the controversial prediction of quantum mechanics that strongly opposed by Einstein. Finally, I will outline the organization of this dissertation.

\subsection{Background and Motivations}

Quantum Information Science (QIS) is an up-and-coming field that exploits the quantum superposition and entanglement by using quantum objects such as atoms, molecules, electrons, photons and phonons [1-7]. Successful implementation of QIS 
has the potential in offering revolutionary applications beyond the capability of classical devices.

In classical treatment of information theory, the fundamental unit of information is a single bit which can have two possible discrete values either 0 or 1 . A single bit can be realized by any two levels physical system such as two distinct voltage or current levels generated by a circuit, two polarization states and etc. In analogues to the classical counterpart, the fundamental unit of quantum information is quantum bit or so called "qubit". A qubit can be implemented by using two levels quantum system such that its state is prepared in superposition of two quantum states. For instance, the state of qubit can be expressed as $|\psi\rangle=\alpha|0\rangle+\beta|1\rangle$, where the probability amplitude $\alpha$ and $\beta$ are the complex numbers that related as $|\alpha|^{2}+|\beta|^{2}=$ 1. The key feature of quantum system is that information can be encoded in superposition of 0 and 1 states. This is in contrast with the classical systems where information must be encoded in one of the two possible states.

In general, major applications of Quantum Information Science are branched into Quantum Computing and Quantum Communication. Quantum Computing is devoted to enhance the computational power by employing quantum phenomena. Several quantum computational algorithms has been indentified to have great advantage over classical computing in certain tasks such as Grover's algorithm in searching in a unsorted database and Shor's algorithm in Fourier transform operation and factoring large number $[\underline{8}, \underline{9}]$. Quantum Communication is an application that involved the transfer of quantum states or information between two distant parties. 
The best known examples in Quantum Communication include Quantum Key Distribution (QKD) and Quantum teleportation [10-14]. Quantum Communication incorporates uncertainty principle, quantum non-cloning theorem and non-locality to allow the detection of eavesdropper, thus provides unconditionally secured communication [15].

Photons are considerably the most promising candidate for the applications of QIS. As photons interact weakly with their environment and relatively robust against environmental disturbance, their quantum mechanical effects such as quantum nonlocality can be well preserved for real world application. Various applications in QIS have been demonstrated by using quantum states of light ranging from quantum imaging and metrology to quantum computing and communication; scaling from single photons to millions of photons $[\underline{12}, \underline{13}, \underline{16-21]}$.

Quantum correlations of photons are often threatened by its deficiencies in preparation, propagation and detection. The preservation of quantum correlations of photons in macroscopic environments will be the key for practical realization of quantum information science. This dessertation is motivated to understand the quantum correlations of photons in macroscopic environments, mainly in concerned with its application in quantum metrology and quantum communications. Nonetheless, the concepts and knowledge developed in this work may have applications in broader context of quantum information processing such as quantum imaging and microscopy with non-classical light $[\underline{22}, \underline{23}]$. 
In quantum metrology, quantum phenomena of light such as entanglement and squeezing are used to enhance the optical phase measurement by suppressing the uncertainty measured physical parameters. The application of quantum metrology can be further extended in surpassing the shot-noise-limited sensitivity for weak force measurements and graviton detection $[\underline{24}, \underline{25}]$. In optomechanics, non-classical light is used in developing sensitive position and momentum detection via macroscopic mechanical correlations measurement [26]. However, the main obstacle for quantum metrology is the difficulty of the experimental realization due to stringent requirement in isolation from environmental noise and delicate control of experiment. Thus, investigating quantum correlation of photons in macroscopic environments is important in exploring the possibility of extracting the quantum correlations of macroscopic object and also the development of quantum devices to achieve unprecedented precision optical phase measurement.

Explosive growth of quantum information science is the main driver of the demand on single photon and entangled photon sources [27]. Quantum states of the single photon in different degree of freedom such as polarization, momentum, energy can be used to encode the qubit for quantum information processing. For quantum key distribution protocols such BB84 and Ekert91, single photon and entangled photon sources are particularly critical to ensure the unconditional security of communication $[\underline{13}, \underline{28}]$. Direct generation of single photon and entangled photon sources in optical fiber is a rather attractive option due to its inherent compatibility with existing fiber optics technologies for practical application. Nevertheless, the 
emergence of differential phase-shift quantum key distribution (DPS-QKD) and decoy-states BB84 protocols relaxing the requirement of single photon source $[\underline{14}$, 29]. As DPS-QKD and decoy-states protocols can be realized by using macroscopic number of photons. Hence investigating bipartite correlations of weak coherent states with the manipulation of linear optics can contribute to the exploration of supplemental resource for realization of DPS-QKD and decoy state BB84 protocols.

Global scale unconditionally secured quantum communication is one of the ultimate goals in quantum information science. Two options are available to achieve long distance quantum communication with current technology; one is through the optical fiber network and another is through free space channel. Even though long distance quantum key distribution in optical fiber and free space channel has been demonstrated $[\underline{30}, \underline{31}]$, the practicality of global scale quantum communication is still in doubt. For free space channel, major limitations are atmospheric scattering, turbulence and propagation losses [32, $\underline{33}]$. Likewise, linear propagation and connection loss with current optical fiber technology limiting the fiber network distance for quantum communication. Hence, quantum correlations of different photons propagate over long distance and lossy environments are great interest from the perspective long distance quantum communication.

In the following section, I will explain how the macroscopic environments are defined in this dessertation. 


\subsection{Macroscopic environments}

According to orthodox interpretation of quantum mechanics- the Copenhagen Interpretation, quantum mechanical description of a large systems will be similar to corresponding its classical description [34]. This is one of the main features of the Copenhagen Interpretation; which appears to be the dividing line between classical (macroscopic) physics and quantum (microscopic) physics. Namely, in microscopic environments events are probabilistic while in the macroscopic environments events appear to be deterministic.

The definition of the macroscopic environments can be extended over a range of physical quantities such as mass, size(volume), spatial separation(length), temperature and number of particles. Well defined criterion to sharply distinguish between macroscopic and macroscopic worlds seems to be an elusive goal. Identification of the macroscopic with the classical physics; and microscopic with the quantum physics has been commonly accepted [35].

However, as a result of advancement in experimental physics, this proposition in defining the boundary of microscopic and macroscopic worlds is proven to be inappropriate and under much scrutiny. Macroscopic objects that are typically welldescribed by classical physics can demonstrate quantum behavior provided it is well isolated from their ambient environment [36]. Recently, much progress has been achieved in demonstrating quantum behavior in macroscopic environment despite its challenging nature. In gravitational wave detection, resonant bar detector that weighs 
few hundred kilograms behaves similarly to a quantum mechanical oscillator $[\underline{37}, \underline{38}]$. In optomechanics, experiments for the demonstration of quantum superposition of two macroscopic masses such as mirrors have been proposed [39]. The mechanical motions of mass have been observed as close to pure quantum states by employing the quantum correlations between the probe light and macroscopic mass [40]. In quantum optics, squeezed states that do not have the classical corresponding counterpart can be prepared with macroscopic numbers of photons [41]. Recently, quantum teleportation has been realized with a new record of 143 kilometers in distance, paving the way for long distance or even global scale quantum communication $[\underline{42}, \underline{43}]$.

Since macroscopic systems are not necessarily classical and it is almost impossible to draw a definite boundary line between macroscopic and microscopic. Hence, macroscopic environment in this work is defined as the environments that cause disappearance of the quantum correlation of a physical system rather than defined on an absolute scale. The macroscopic environments that we explore in this dissertation include noise photon in the light source, macroscopic photons number, spatial separation of the photons, macroscopic mirror, loss and dynamics of propagation channel, intrinsic noise in detection and so on.

\subsection{Quantum Superposition and Entanglement}

Principle of Superposition is a fundamental property of physic which also lay down the cornerstone for peculiar quantum phenomena. Specifically, provided that the 
wave functions $\left|\psi_{1}\right\rangle$ and $\left|\psi_{2}\right\rangle$ both satisfy the Schrödinger equation for a physical system, then the linear superposition of the wave functions given as $|\psi\rangle=\alpha_{1}\left|\psi_{1}\right\rangle+$ $\alpha_{2}\left|\psi_{2}\right\rangle$ also satisfies the Schrödinger equation. The complex numbers $\alpha_{1}$ and $\alpha_{2}$ are the probability amplitude of the wave functions. Quantum Superposition can be further generalized to say that the physical states of a quantum system can be described by superposition of more than one wave function instead of a single wave function. The superposition of the wave functions is written as $|\psi\rangle=\sum_{i} \alpha_{i}\left|\psi_{i}\right\rangle$, where the probability of finding the system in particular stats $\left|\psi_{i}\right\rangle$ is $\left|\alpha_{i}\right|^{2}$. Quantum Superposition evidences to probabilistic nature of quantum mechanics. In addition, it also holds the key for quantum information science as fundamental unit of quantum information is solely based on the superposition of quantum states.

As a result of quantum superposition, entanglement is the most intriguing and counterintuitive concept in quantum mechanics. A two-particle system with wave function that cannot be factorized into a product state of individual wave functions is the simplest form of entangled states. Considering a physical system emitting a correlated pair of photons with the probability of having the individual photon in horizontal $(\mathrm{H})$ or vertical $(\mathrm{V})$ polarization states is $50 \%$ each and the occurrence is random. The entangled states of this system can be written as

$$
\begin{aligned}
& \left|\psi^{ \pm}\right\rangle=\frac{1}{\sqrt{2}}\left(\left|H_{1} H_{2}\right\rangle \pm\left|V_{1} V_{2}\right\rangle\right) \\
& \left|\phi^{ \pm}\right\rangle=\frac{1}{\sqrt{2}}\left(\left|H_{1} V_{2}\right\rangle \pm\left|V_{1} H_{2}\right\rangle\right)
\end{aligned}
$$


These are four correlated and anti-correlated Bell's states with the subscripts referring to each individual photon of photon pair.

Assuming that the system is in entangled state $\left|\psi^{+}\right\rangle$, when photon 1 is detected horizontal polarization states, $H_{1}$ the wave function of entangled state collapsed into the product state $\left|H_{1} H_{2}\right\rangle$. Therefore the photon 2 must be in the horizontal polarization states, $H_{2}$ even if no measurement is made, and vice versa if photon 1 in vertical polarization states. Similar, same explanation can be applied to all other entangled states $\left|\psi^{-}\right\rangle,\left|\phi^{+}\right\rangle$and $\left|\phi^{-}\right\rangle$. This implies that even though polarization measurement results of photon 1 is random and unpredictable; they allow us to predict the results of polarization measurement of photon 2 with absolute accuracy. Surely, quantum entanglement is not limited to two particles system. For instance, Greenberger-Horne-Zeilinger state which is a polarization entangled state involving more than 2 photons that proposed and demonstrated by Zeilinger's group [44-46]. In fact, it is predicted that multi-particle entanglement to offer richer nonclassical correlations and access to more intriguing applications [47]. In addition, entanglement with different degree of freedom such as momentum entanglement, time-energy entanglement, and polarization entanglement or entanglements with multi degrees of freedom such as Hyper-entanglement have been demonstrated [4851].

The term entanglement (Verschränkung in German) was first introduced by Schrödinger when he uses the famous "Schrödinger's cat" to illustrate entangled states, which was mystifying during that time. This thought experiment pointed out 
that phenomenon of entanglement clearly contradicting the common belief as a cat cannot be dead or alive at the same time. Schrödinger did made a good point with this example as this perplexing paradox rarely observed in macroscopic world, because quantum systems interact with the noisy macroscopic environment and lose their correlations. The concept of entanglement was not accepted plainly by physicists. Its debate can be traced back to as early as 1935 when Einstein, Podolsky, and Rosen published a paper on EPR paradox [52]. The EPR trio illustrated a quantum system that consists of two spatially separated particles where the position/momentum measurement of either particle would instantly determine the position/momentum measurement outcome of the other particle. They saw that the instantaneous influence of one particle on another one as a superluminal effect which is forbidden by special relativity and called it as "spooky action at a distance". As a result, they went on to claim that the Copenhagen interpretation on quantum theory is incomplete and "local hidden variables" are required to fully describe physical state of a system [52]. Several months later, Bohr reply to EPR paper by arguing that EPR treatment of two-particles quantum system is inappropriate as they assumption on locality means that an act of measurement on particle 1 (which is a part of that particular quantum system) will not disturb the quantum system [53]. Then, Bohr went further to insist that quantum mechanics is complete.

In 1964, John Bell derived the famous Bell's theorem and formulated Bell's inequality [54]. According to Bell's theorem, if the correlation of two particles can be described by "local hidden variables" then the Bell's inequality is always obeyed. In 
contrast, Bell's inequality would be violated if Quantum mechanics prevails and its interpretation on non-locality of EPR paradox is true. Bell's theorem is significant as it shows that "local hidden variables" cannot reproduce the prediction by Quantum mechanics on about strongly correlated two-particle system. The emergence of the Bell's theorem established the benchmark to experimentally test the existence of “local hidden variables". Indeed, Bell's work brings hopes in concluding EinsteinBohr philosophical exchange on quantum theory and verifies the concept of quantum entanglement. In 1969, John Clauser and his co-worker proposed an experiment to test a more generalized "local hidden variables" theory with a modified Bell's inequality (which is much known as $\mathrm{CHSH}$ inequality) by considering actual experimentation system [55]. Since then, all experimental results astonishingly pointed to the triumph of Quantum mechanics [ㄷ-58].

\subsection{Dissertation Organization}

Most of the materials presented in this dissertation have been published in journal article or is in the peer review process for publication. The presentation style, contents and figures in this manuscript in each chapter are often extracted from a published or in-review paper. Further elaboration and proper modifications are done to ensure the consistency of presentation style, notation and references so that the dissertation can be read fluently.

This dissertation is organized as follows: In chapter 2, we present a measurement scheme for observing quantum correlations and entanglement in the 
spatial properties of two macroscopic mirrors by employing single spatial compass states through direct propensity measurement. Chapter 3 , we demonstrated that intrinsic quantum correlations of weak coherent states are observed between two parties over a distance of $10 \mathrm{~km}$ transmission fiber through a novel detection scheme. For chapter 4, we generate polarization correlated and entangled photon pair at telecom wavelength through spontaneous four-wave mixing process in a short $10 \mathrm{~m}$ of highly nonlinear fiber. Following in chapter 5, we present a study on quantum correlation and interference of fiber based photon-pairs with one photon experiencing standard loss or multiple scattering in a random medium. Finally, the summary and outlook of this work will be rolled out in chapter 6 . 


\section{Chapter 2}

\section{Macroscopic Mechanical Correlations of Two Mirrors ${ }^{1}$}

\subsection{Overview}

In this chapter, we propose a measurement scheme for observing quantum correlations and entanglement in spatial properties of two macroscopic mirrors using single photons spatial compass states.

First, we begin with a short introduction on some previous studies on the macroscopic mechanical correlations and quantum decoherence by using nonclassical states such as quantum superposition of coherent states, entanglement and squeezed states of light. In addition, we will discuss the Wodkiewicz's idea of measuring two non-commuting variables of quantum states with propensity. Direct measurement of propensity or operational probability density distribution can be employed to study the mechanical correlations of the two mirrors [두].

\footnotetext{
${ }^{1}$ The material contained in this chapter was previously published in Physical Review A.
} 
Then, we discuss the properties of the Wigner phase space distribution about negativity of Wigner function in certain regions of the phase space distribution. The wave-particle duality characteristic of the Wigner function allows us to characterize spatial compass states that are generated from the superposition of single Gaussian transverse electromagnetic (TEM) 00 mode of single photons in phase space distribution.

An experiment setup with realistic parameters is proposed for the generation of spatial compass states. Two spatial versions of compass states are generated by using single Gaussian mode of single photons in a simple interferometer. The detail of proposed experiment will be discussed thoroughly and the phase space distribution features of spatial compass states such as chessboard pattern will be illustrated by using Wigner function.

In next section, we will discuss the concept of propensity $\mathcal{P}_{b}\left(d_{x}, d_{p}\right)$ and how to carry out measurement of propensity in our proposed experiment. In addition, we will reveal the relation between the Wigner function and propensity. Then, we follow up with explanations on the dynamical process of propensity and the realization Einstein-Podolsky-Rosen (EPR) correlation in two mirrors' experimental coordinates. The displacement and tilt correlations of the two mirrors are manifested by single photons and projection measurements through a measuring device which measures the propensity of the compass states. The technique can extract mechanical correlations of the two mirrors and lock them into the EPR correlation by the means of maintaining maximum propensity. Furthermore, we calculated the variances in 
position and momentum of the proposed imaging system and visualize the EPR entanglement region. The criteria for EPR entanglement of these mirrors can also verified by sub-Planck structures in the propensity.

We then formulate the discrete-like property of the propensity $\mathcal{P}_{b}(m, n)$. The discrete phase-space spot in $\mathcal{P}_{b}(m, n)$ can be used to explore environmental perturbed quantum jumps of the EPR correlations in phase space, hence, enables discrete phase-space quantum computing and information processing.

\subsection{Introduction}

Entanglement between macroscopic oscillators has drawn much attention recently because its physics can help us to study decoherence in quantum systems and enhance robustness in quantum metrology [60-62]. The classical and quantum decoherence associated with environmental perturbation on a quantum system can be determined using Wigner function through its sub-Planck phase-space structure. Zurek has proposed a compass state based on superpositions of coherent states to explore the orthogonality between perturbed and unperturbed quantum states [63]. The idea is then further pursued for achieving Heisenberg-limited sensitivity in weak force measurements by motional degree of freedom of a trapped ion [25, $\underline{64}]$. Mechanical correlations for macroscopic object such as cooled mirror and membrane have been observed in the area of optomechanics for developing sensitive position and momentum measurements [65-68]. In quantum optics, entanglement and squeezing are often the quantum tools that used to surpass the standard quantum limit 
(SQL) via quantum nondemolition measurements and the backaction-evasion technique of measuring devices [69-71].

The concept of propensity was first proposed by Wodkiewicz for measuring two non-commuting observables of a quantum state simultaneously with realistic motions of a measuring device [72]. The propensity or operational probability density distribution is the convolution (overlapping) of the Wigner function of the detected state and the Wigner function of the filtering state provided by the measuring device. If the detected and the filtering states are the identical quantum state, then the propensity will provide the information of orthogonality between the quantum state and its phase-space-disturbed quantum state. The propensity is the generalized function of the two-particle Wigner function wherein there are no correlations between the particles, i.e., by means of the product of two single particle Wigner functions [73]. With the help of the spatial compass state, single photons, and the dynamical process of measuring propensity, our phase-space imaging system can project the interference terms (chessboard pattern) of the product Wigner function through direct measurement. This leading to the observation of spatial EinsteinPodolsky-Rosen (EPR) correlations of two mirrors in contrast to the case considered by O'Connell and Walls [73].

\subsection{Wigner Function}

Given that spatial compass state is proposed to observe the spatial EPR correlations of two mirrors, one would like to visualize and characterize its properties. In this 
work, we explore the phase space properties of the spatial compass state by using the Wigner function.

Wigner function is a quasiprobability distribution function in phase space (position and momentum) that was originally introduced by Eugene Wigner in 1932, where quantum corrections to classical statistical mechanics were of interest [74]. To date, Wigner function founds plenty of applications in both classical and quantum optics [75-78]. The Wigner distribution $\mathcal{W}(x, p)$ for a one dimensional spatial wave function $\psi(x)$ is defined as:

$$
\mathcal{W}(x, p)=\frac{1}{2 \pi} \int_{-\infty}^{\infty} d \varepsilon e^{-i \varepsilon p} \psi^{*}\left(x+\frac{\varepsilon}{2}\right) \psi\left(x-\frac{\varepsilon}{2}\right)
$$

Here $x$ indicates position and $p$ indicates momentum in phase space.

In classical mechanics, state of a physical system can be characterized by its phase space trajectory and the possible states of the physical system are uniquely represented in the phase diagram. Given an ensemble of similarly prepared physical system, the probability of finding a physical system in a particular state (position and momentum pair) is described by using classical phase space density function. Naturally one might consider Wigner function $\mathcal{W}(x, p)$ to have similar characteristics as classical phase space density function. However, in contrast to classical probability density function, the Wigner function may exhibits negative values at certain domains and thus cannot be interpreted as a probability function. In fact, only the Gaussian Wigner function illustrates positive definite distribution as shown in Fig. 2.1 [구]. 

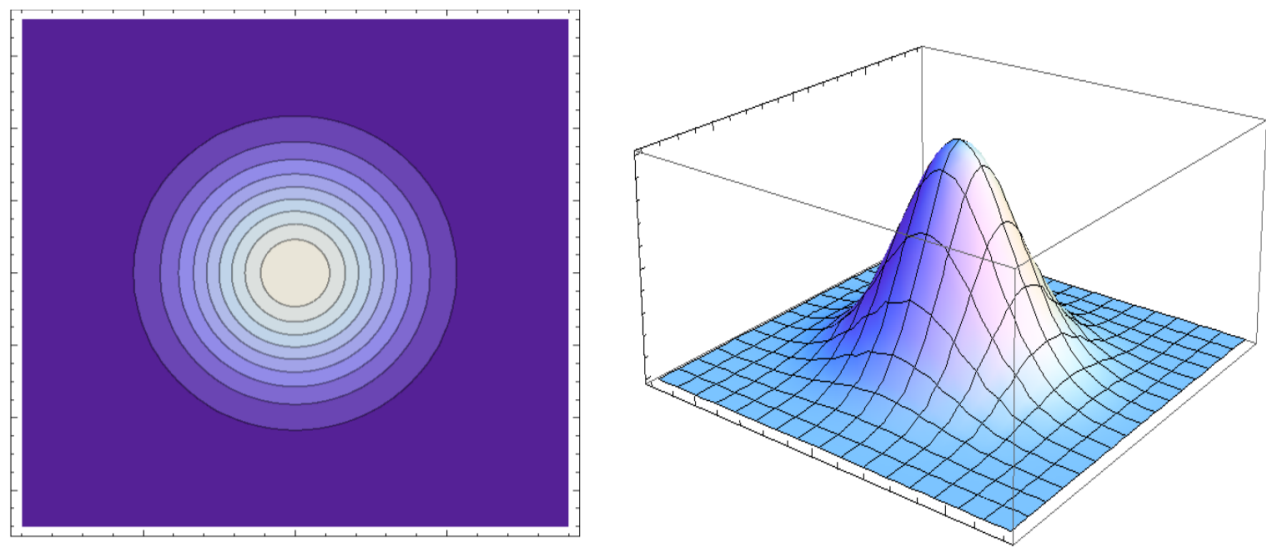

Figure 2.1. Gaussian Wigner function: (Left) 2 dimensional contour plot; (Right) 3dimensional contour plot.

The distinctive feature of negativity in Wigner function stirred up considerable interest and was exploited by physicists to explore the nonclassical properties of light such as entanglement and squeezing [80-82]. However, the negativity in Wigner function is not an exclusive signature of the non classical property of light. In fact, negative values in the Wigner function of the classical field has been demonstrated; simulating the quantum properties of light in its own right [76]. The negative value of the Wigner function for the classical field represents destructive phase space interference in terms of classical waves optics, in analogy to nonclassical properties of light [르.

The wave-particle duality characteristic of the Wigner function is described in its definition in Eq. 2.1. The particle-like behavior of the Wigner function arises from position and momentum domain in its definition, which characterize the particle characteristics of the light by the means of geometrical ray optics. On the other hand, the wave-like behavior of the Wigner function originates from the spatial wave 
function $\psi(x)$ in its definition which is a solution of wave equation. The wave-like feature of Wigner function lays down the foundation for us to explore the properties of spatial compass state in this work. The spatial compass state is constructed from the coherent interference structures from a pair of transverse electromagnetic (TEM) $)_{00}$ mode of single photons in phase space. Furthermore, the wave-particle duality characteristic of the Wigner function provides complete information on the properties of wave functions and related mutual coherence function [ㅁ3]. The interesting wave-particle duality features of Wigner function has also been used to study coherence and propagation of light in different propagation media [ㄴ-86].

\subsection{Single Photons Spatial Compass state}

In this work, we propose to generate two spatially separated spatial compass states, $\odot_{1}$ and $\bigodot_{2}$ using a single Gaussian transverse electromagnetic $(\mathrm{TEM})_{00}$ mode of single photons through a simple interferometer. These spatial compass states are displaced and tilted by classical and quantum spatial fluctuations of two independent mirrors. Then, the spatial compass states are directed into an imaging system which performs projection measurements on spatial compass states with a single-photon detector. The proposed experimental setup is shown in Fig.2.2. 


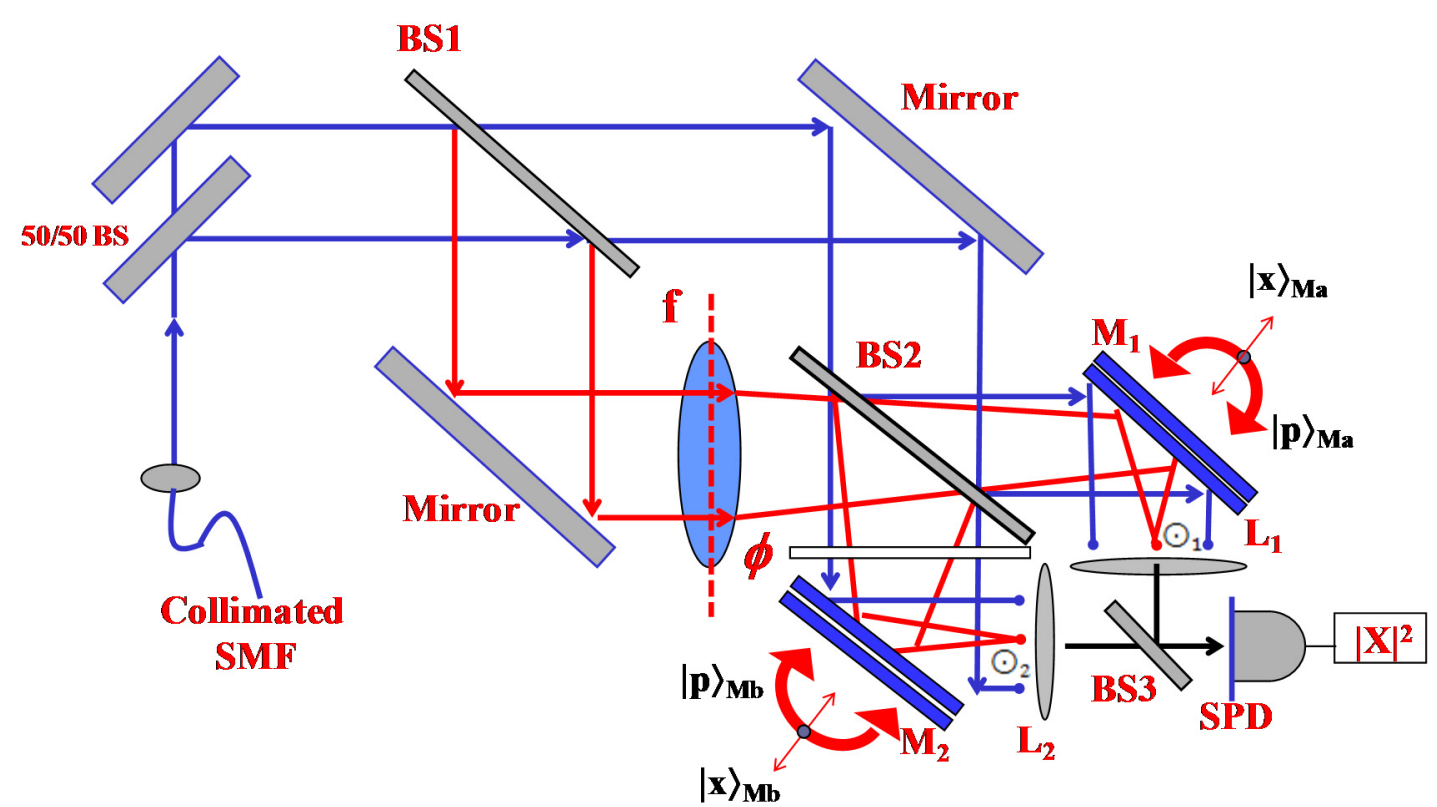

Figure 2.2. The proposed experimental setup for measuring the propensity of two spatial compass states and spatial correlations of two mirrors. ( $\mathrm{SMF}=$ single mode fiber, $\mathrm{BS}=$ beam splitter, $\mathrm{L}=$ lense, $\mathrm{M}=$ mirror, $\mathrm{SPD}=$ single photon detector)

A well defined and collimated single Gaussian TEM00 beam of single photons is obtained from a collimated single-mode fiber (SMF). The spatial wave function $\psi(x)$ of the TEM 00 beam is given as,

$$
\psi(x) \propto \exp \left[-\frac{x^{2}}{2 \sigma_{a}^{2}}\right]
$$

where $\sigma_{a}$ is the spatial width of the SMF. Then, the TEM00 beam is split into two beams with the distance between them about $2 a$ creating two spatially separated TEM 00 beam with wave function,

$$
\psi(x) \propto \exp \left[-\frac{(x+a)^{2}}{2 \sigma_{a}^{2}}\right]+\exp \left[-\frac{(x-a)^{2}}{2 \sigma_{a}^{2}}\right]
$$


The wave function $\psi(x)$ is split into two components by using a 50-50 beam splitter (BS1). The reflected wave function is denoted as $\psi^{r}(x)$ and the transmitted wave function is denoted as $\psi^{t}(x)$. Then, the reflected wave function at $\operatorname{BS} 1 \psi^{r}(x)$ is Fourier transformed to

$$
\psi^{r}(p) \propto \exp \left[-\frac{p^{2} \sigma_{a}^{2}}{2}\right] \cos (p a)
$$

in the Fourier plane through a lens with a focal length of $f$. The derivation of this Fourier transform is given in Appendix A. $\psi^{r}(p)$ is then combined with the transmitted wave function $\psi^{t}(x)$ through another $50-50$ beam splitter (BS2), creating the superposition

$$
\bigodot_{1} \propto \psi_{1}^{t}(x)+\psi_{1}^{r}(p)
$$

which is called the spatial compass state at output 1 of BS2. We generate two spatially separated spatial compass sates, $\bigodot_{1}$ and $\odot_{2}$, at each output of the beam splitter (BS2) as shown in Fig. 2.2.

The second compass state at output 2 of BS2 is given as

$$
\bigodot_{2} \propto \psi_{2}^{t}(x)+\exp [i \phi] \psi_{2}^{r}(p)
$$

where the relative phase $\phi$ is provided by a linear phase shifter to compensate the phase difference between $\psi_{2}^{t}(x)$ and $\psi_{2}^{r}(p)$. Ideally, we can generate the spatial compass states, $\bigodot_{1}$ and $\bigodot_{2}$ to be exactly identical. 
The wave function $\psi_{1,2}^{r}(p)$ in the Fourier plane can be rewritten in position coordinates as

$$
\psi_{1,2}^{r}(x) \propto \exp \left[-\frac{x^{2}}{2 \sigma_{b}^{2}}\right] \cos \left(x D_{p}\right)
$$

where we have used the substitutions of $p=k \theta=\frac{k x}{f}, D_{p}=\frac{k a}{f}$, and $\sigma_{b}^{2}=\frac{f^{2}}{2 k^{2} \sigma_{a}^{2}} \cdot \theta$ is the incident angle of the photons with respect to optical axis and $k=\frac{2 \pi}{\lambda}$ is the wave vector. With the help of the phase shifter to set $\phi=0$, the spatial compass states $\bigodot_{1}$ and $\bigodot_{2}$ can be written in position coordinates in a identical form as

$$
\begin{aligned}
\odot_{1,2} & \propto \psi_{1,2}^{t}(x)+\psi_{1,2}^{r}(x) \\
& \propto \exp \left[-\frac{(x+a)^{2}}{2 \sigma_{a}^{2}}\right]+\exp \left[-\frac{(x-a)^{2}}{2 \sigma_{a}^{2}}\right]+\exp \left[\frac{x^{2}}{2 \sigma_{b}^{2}}\right] \cos \left(x D_{p}\right) .
\end{aligned}
$$

We explore the spatial compass states by using the Wigner function as given by

$$
\mathcal{W}(x, p)_{1,2} \propto \int_{-\infty}^{\infty} d \varepsilon e^{-i \varepsilon p} \bigodot_{1,2}{ }^{*}\left(x+\frac{\varepsilon}{2}\right) \odot_{1,2}\left(x-\frac{\varepsilon}{2}\right)
$$

By substituting the $\bigodot_{1}$ in Eq. 2.8 to Eq. 2.9, we obtain the Wigner function is such a way that,

$$
\begin{aligned}
\mathcal{W}(x, p)_{1,2} \propto & \int_{-\infty}^{\infty} d \varepsilon e^{-i \varepsilon p} \psi_{1,2}^{* t}\left(x+\frac{\varepsilon}{2}\right) \psi_{1,2}^{t}\left(x-\frac{\varepsilon}{2}\right) \\
& +\int_{-\infty}^{\infty} d \varepsilon e^{-i \varepsilon p} \psi_{1,2}^{* r}\left(x+\frac{\varepsilon}{2}\right) \psi_{1,2}^{r}\left(x-\frac{\varepsilon}{2}\right) \\
& +\int_{-\infty}^{\infty} d \varepsilon e^{-i \varepsilon p} \psi_{1,2}^{* t}\left(x+\frac{\varepsilon}{2}\right) \psi_{1,2}^{r}\left(x-\frac{\varepsilon}{2}\right)
\end{aligned}
$$




$$
+\int_{-\infty}^{\infty} d \varepsilon e^{-i \varepsilon p} \psi_{1,2}^{* r}\left(x+\frac{\varepsilon}{2}\right) \psi_{1,2}^{t}\left(x-\frac{\varepsilon}{2}\right)
$$

The Wigner functions for the generated spatial compass states are shown in Fig. 2.4. Spatial Wigner function that we obtain has almost similar structure as the compass state in the coherent state representation proposed by Zurek [63]. The chessboard pattern of the Wigner function is composed from the interference of components $\psi_{1,2}^{* t}\left(x+\frac{\varepsilon}{2}\right) \psi_{1,2}^{t}\left(x-\frac{\varepsilon}{2}\right)$ and $\psi_{1,2}^{* r}\left(x+\frac{\varepsilon}{2}\right) \psi_{1,2}^{r}\left(x-\frac{\varepsilon}{2}\right)$ of the compass states. The Wigner functions of these two components are given by

$$
\begin{aligned}
\mathcal{W}_{1,2}(x, p)^{t t} & \propto \int_{-\infty}^{\infty} d \varepsilon e^{-i \varepsilon p} \psi_{1,2}^{* t}\left(x+\frac{\varepsilon}{2}\right)+\psi_{1,2}^{t}\left(x-\frac{\varepsilon}{2}\right) \\
& \propto \exp \left[-\frac{(x+a)^{2}}{\sigma_{a}^{2}}-p^{2} \sigma_{a}^{2}\right]+\exp \left[-\frac{(x-a)^{2}}{\sigma_{a}^{2}}-p^{2} \sigma_{a}^{2}\right] \\
& +2 \exp \left[-\frac{x^{2}}{\sigma_{a}^{2}}-p^{2} \sigma_{a}^{2}\right] \cos (2 p a)
\end{aligned}
$$

and

$$
\begin{gathered}
\mathcal{W}_{1,2}(x, p)^{r r} \propto \int_{-\infty}^{\infty} d \varepsilon e^{-i \varepsilon p} \psi_{1,2}^{* r}\left(x+\frac{\varepsilon}{2}\right)+\psi_{1,2}^{r}\left(x-\frac{\varepsilon}{2}\right) \\
\propto \exp \left[-\frac{(x)^{2}}{\sigma_{b}^{2}}-\left(p+D_{p}\right)^{2} \sigma_{b}^{2}\right]+\exp \left[-\frac{(x)^{2}}{\sigma_{b}^{2}}-\left(p-D_{p}\right)^{2} \sigma_{b}^{2}\right] \\
+2 \exp \left[-\frac{(x)^{2}}{\sigma_{b}^{2}}-p^{2} \sigma_{b}^{2}\right] \cos \left(2 x D_{p}\right)
\end{gathered}
$$


respectively. The cosine terms in Eq. 2.11 and Eq. 2.12 indicating interference of the spatial wave functions due to the wave properties of single photons. Plots of $\mathcal{W}_{1,2}(x, p)^{t t}$ and $\mathcal{W}_{1,2}(x, p)^{r r}$ are depicted in Fig. 2.3 (a) and (b).

The summation of the interference terms $\exp \left[-\frac{x^{2}}{\sigma_{a}^{2}}-p^{2} \sigma_{a}^{2}\right] \cos (2 p a)$ and $\exp \left[-\frac{(x)^{2}}{\sigma_{b}^{2}}-p^{2} \sigma_{b}^{2}\right] \cos \left(2 x D_{p}\right)$ will produce the chessboard pattern in the Wigner function of compass states as shown in Fig. 2.4. Spatial frequencies of both interference terms are proportional to $a$ and $D_{p}$ respectively. Thus, the distance $a$ in $\mathcal{W}(x, p)_{t t}$ will determine the sensitivity for measuring the momentum or tilt of the mirrors while the parameter $D_{p}$ in $\mathcal{W}(x, p)_{r r}$ determine the sensitivity for measuring the position of the mirrors. Note that the position and momentum coordinates of the chessboard pattern in the Wigner function do not directly correspond to real physical spatial coordinates of the proposed imaging system.

In addition to chessboard pattern which is the primary interest in this work. The remaining two components, $\psi_{1,2}^{* t}\left(x+\frac{\varepsilon}{2}\right)+\psi_{1,2}^{r}\left(x-\frac{\varepsilon}{2}\right)$ and $\psi_{1,2}^{* r}\left(x+\frac{\varepsilon}{2}\right)+$ $\psi_{1,2}^{t}\left(x-\frac{\varepsilon}{2}\right)$ contribute to the four corner fringes that exist between two lumps of $\mathcal{W}(x, p)_{a a}$ and $\mathcal{W}(x, p)_{b b}$ as depicted in Fig.2.5. These corner fringes are due to the superposition of spatial wave functions in a similar way as the interference structure of two spatial wave functions as seen in Fig. 2.3. Derivation on the Wigner distribution of Spatial Compass state is given in Appendix C. 
(a)
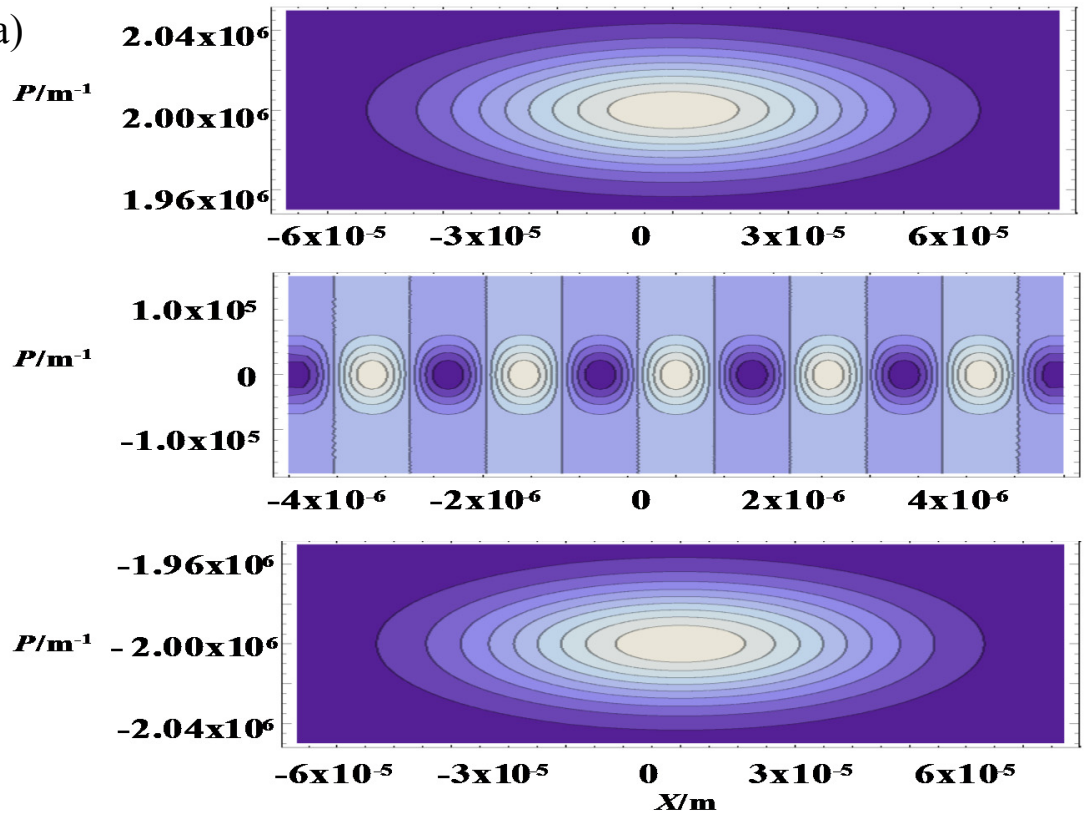

(b)
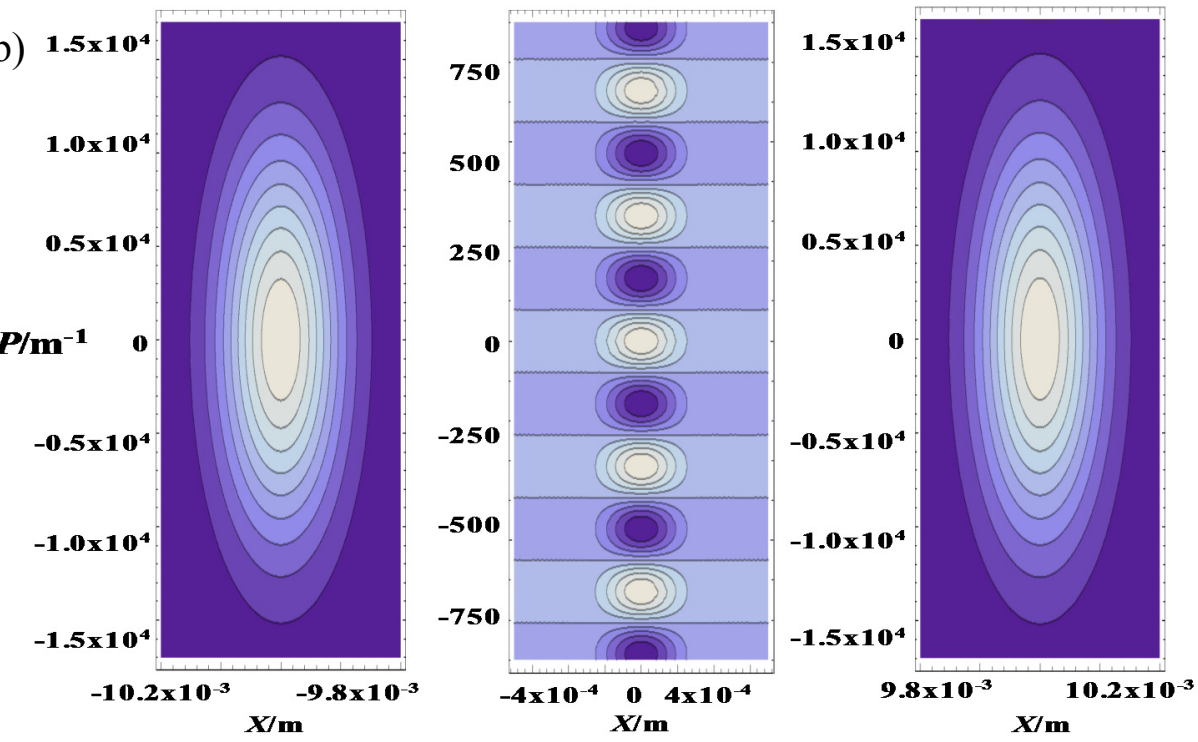

Figure 2.3. The phase space plots of a spatial compass state with realistic parameters: $\sigma_{a}=100 \mu \mathrm{m}, \mathrm{a}=1.0 \mathrm{~cm}, \mathrm{f}=5.0 \mathrm{~cm}$, and $\lambda=632 \mathrm{~nm} . \mathcal{W}_{1,2}(x, p)^{t t},(\mathrm{~b}) \mathcal{W}_{1,2}(x, p)^{r r}$ 


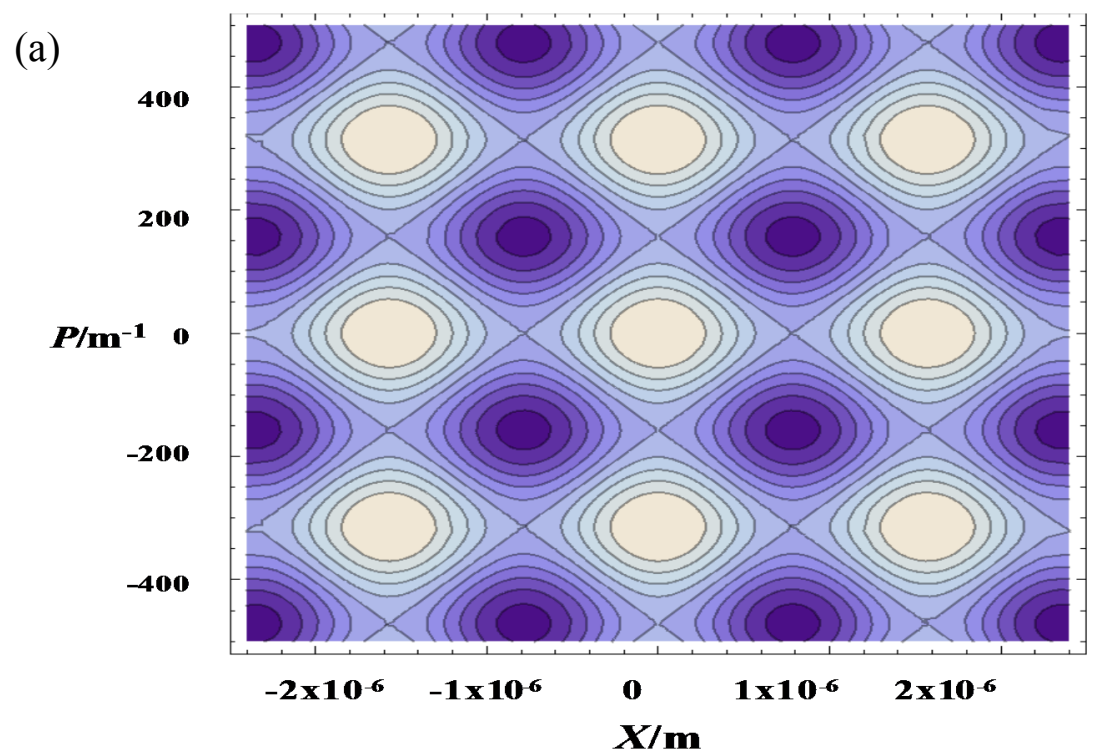

(b)

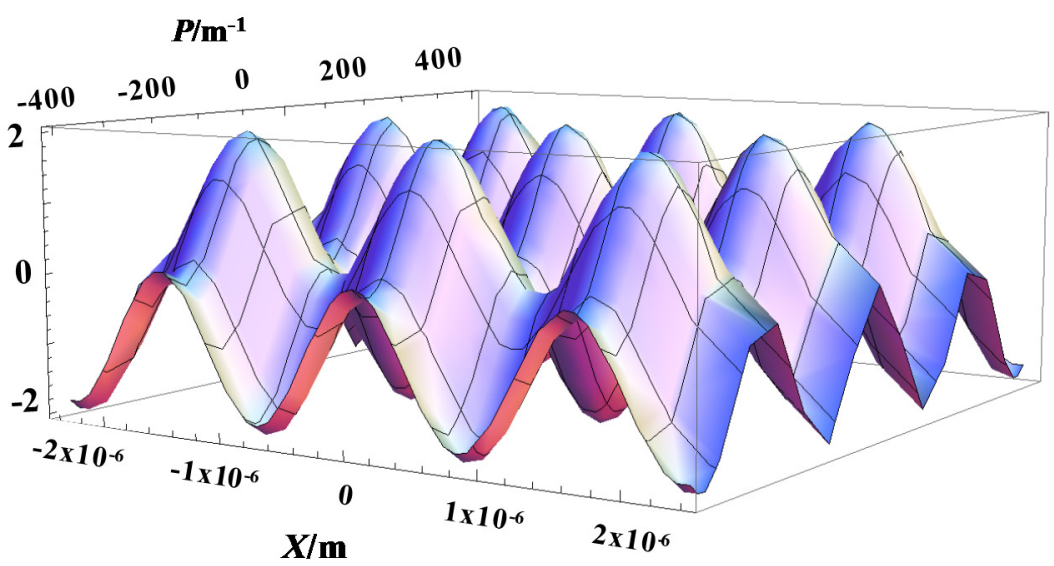

Figure 2.4. The phase space plots of a spatial compass state with realistic parameters: $\sigma_{a}=100 \mu \mathrm{m}, \mathrm{a}=1.0 \mathrm{~cm}, \mathrm{f}=5.0 \mathrm{~cm}$, and $\lambda=632 \mathrm{~nm}$. (a) 2 dimension plot of chessboard pattern, (b) 3 dimension plot of chessboard pattern 

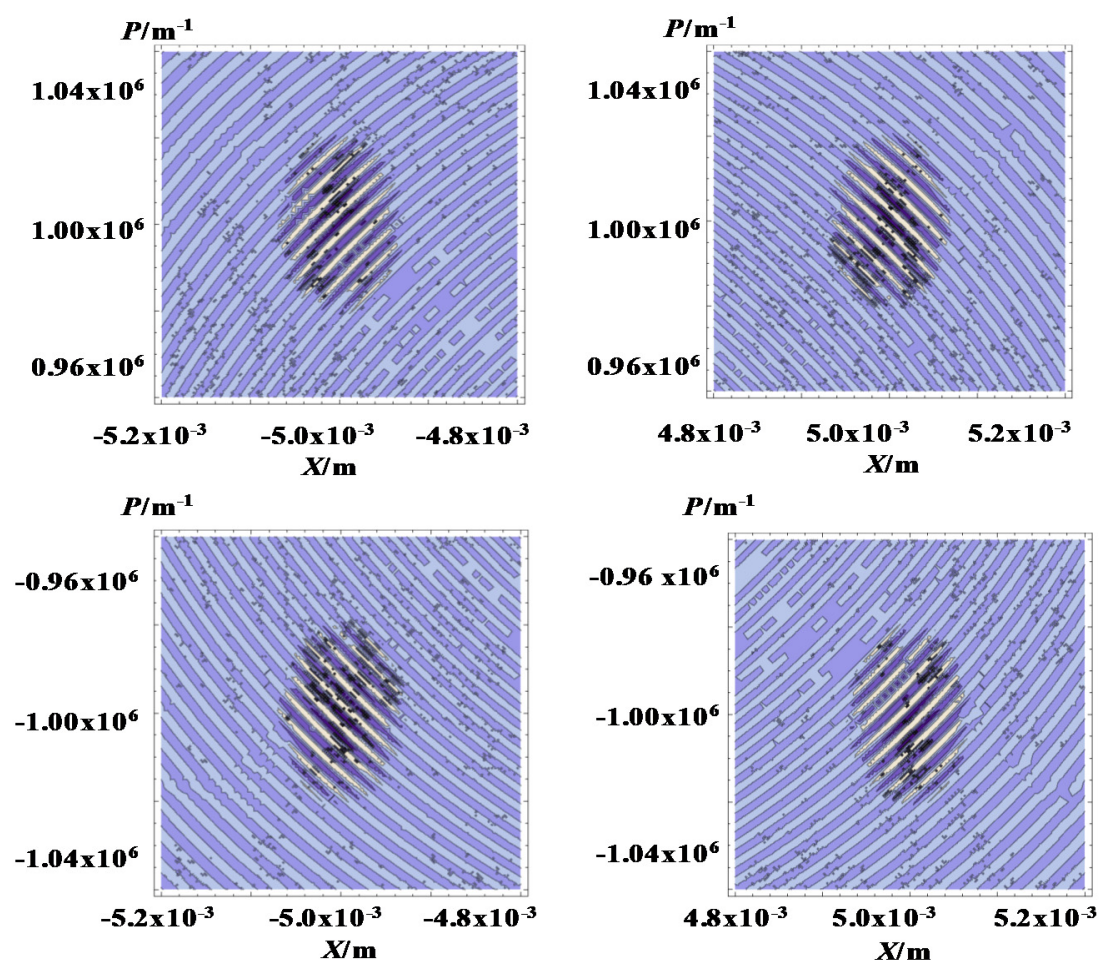

Figure 2.5. Phase space distribution of the four corner fringes between the two lumps of $\mathcal{W}(x, p)_{t t}$, and $\mathcal{W}(x, p)_{r r}$

\subsection{Propensity}

Since we use single photons for generating the spatial compass states, the Wigner distribution corresponding to probability for the photon to be at the center of the chessboard is low, and hence, the probability of detecting the photon at that spot is extremely low. To enhance the probability of finding the photon at the center of the chessboard, we use an imaging system $\left(L_{1}, L_{2}\right)$ for collecting the entire $\bigodot_{1,2}$ beam or single photons into a single-photon detector while selectively projecting the position and momentum of the center spot into the detector. The imaging system has been used to measure a smoothed Wigner function and a true Wigner function including the sub-Planck structure of an optical beam $[\underline{76}, \underline{87]}$. 
As shown in Fig.2.2, each spatial compass state passes through the lenses $\left(L_{1}, L_{2}\right)$ and obtains the quadrature-phase term $\exp \left[i \frac{k x^{2}}{2 f}\right]$. Then, the spatial compass states will be collected on the single-photon detector. The detector measures the interference of these spatial compass states as a function of the relative displacement $d_{x}$ and momentum or tilt $\delta p=\frac{k d_{p}}{f}$, where $d_{p}$ is the displacement associated with the tilt or the displacement of the lenses The interference signal as a function of $d_{x}$ and $d_{p}$ is given by

$$
V_{B}\left(d_{x}, d_{p}\right) \propto \frac{k}{f} \exp \left(i \frac{\mathrm{k} d_{p}^{2}}{2 f}\right) \int_{-\infty}^{\infty} d x^{\prime} \exp \left(i \frac{\mathrm{k} x^{\prime} d_{p}}{f}\right) \odot_{2}{ }^{*}\left(x^{\prime}-d_{x}\right) \odot_{1}\left(x^{\prime}\right)
$$

The relative displacement and momentum or tilt can be caused by random spatial fluctuations of the mirrors at temperature $T$ that can be controlled or locked by moving lenses and mirrors. Applying the squarer $X^{2}$ after the single-photon detector, the squared modulus interference signal (propensity) is given by

$$
\begin{aligned}
\left|V_{B}\left(d_{x}, d_{p}\right)\right|^{2} & =\mathcal{P}_{b}\left(d_{x}, d_{p}\right) \\
& \propto \int_{-\infty}^{\infty} d x d p \mathcal{W}\left(x-d_{x}, p+\frac{k d_{p}}{f}\right)_{\odot_{2}} \mathcal{W}(x, p)_{\odot_{1}}
\end{aligned}
$$

Eq. 2.14 essentially lays out the relation between the Wigner function and realistic phase-space probability distribution, which can be obtained directly from the measurement of $\left|V_{B}\left(d_{x}, d_{p}\right)\right|^{2}$. The value of propensity at $\mathcal{P}_{b}\left(d_{x}, d_{p}\right)$ is obtained by displacing the origin of $\mathcal{W}_{\odot_{2}}$ to that particular point $\left(d_{x}, d_{p}\right)$, multiplying by $\mathcal{W}_{\odot_{1}}$ and then integrating over both $x$ and $p$. This means that the propensity is a positive 
definite phase-space probability distribution that measures the overlap (convolution) of $\mathcal{W}_{\odot_{2}}$ and $\mathcal{W}_{\odot_{1}}$. Detail derivation of the relation between the Wigner function and $V_{B}\left(d_{x}, d_{p}\right)$ in Eq. 2.13 and Eq. 2.14 is given in Appendix B.

In accordance with concept of propensity that proposed by Wodkiewicz, the detected state $\odot_{1}$ is associated with mirror 1 , and the filtering state $\odot_{2}$ associated with mirror $2\left[\underline{72}, \underline{88]}\right.$. The filtering state $\odot_{2}$ has the functionality of resolving the position and momentum of the detected state $\bigodot_{1}$. Through the chessboard pattern, the filtering state $\odot_{2}$ can provide sub-Planck phase-space resolution to map out the detected state $\odot_{1}$ [25]. Direct measurement of the propensity provides the information on the orthogonality of the perturbed and unperturbed spatial compass states. By using the imaging system, we can project the center of the chessboard for the maximum beat of $\mathcal{P}_{b}\left(d_{x}, d_{p}\right)$ at around $d_{x} \sim 0$ and $d_{p} \sim 0$. The spatial coordinates of the chessboard pattern in the propensity exactly correspond to the coordinate system of the imaging system. To illustrate the dynamical process of the propensity extracting the EPR correlations of two mirrors, we discuss the convolution process in Eq. 2.14 by using the physical transformations

$$
\begin{aligned}
& x_{b}=x-d_{x}=x_{a}-d_{x} \\
& p_{b}=p+\frac{k d_{p}}{f}=p_{a}+\frac{k d_{p}}{f}
\end{aligned}
$$

in the imaging system. The system is initially at the common zero position and momentum, $\bigodot_{1}$ is projected to $x_{a}=0$ and $p_{a}=0$, and similarly, $\bigodot_{2}$ is projected to 
$x_{b}=0$ and $p_{b}=0$. If mirror 1(2) moves to the position $x_{a}^{\prime}=0-d_{x}=a$, mirror 2(1) must move to $x_{b}^{\prime}=0-d_{x}=-a$ in order to maintain the maximum beat signal $\mathcal{P}_{b}\left(d_{x}, d_{p}\right)$ as shown in Fig. 2.6 so that $x_{a}^{\prime}+x_{b}^{\prime}=0$ is fulfilled. This is due to the fact that the system has to make sure the detected state overlaps with the filtering state in the detector plane.

As for the momentum, if mirror 2(1) is tilted at the angle $p_{a}^{\prime}=0+k \theta$ mirror 1(2) must tilt to $p_{b}^{\prime}=0+k \theta=0+\frac{k d_{p}}{f}$, or the lens $\mathrm{L}_{1}\left(\mathrm{~L}_{2}\right)$ must move up $d_{p}$ in order to maintain the maximum beat $\mathcal{P}_{b}\left(d_{x}, d_{p}\right)$ as shown in Fig.2.7 so that $p_{a}^{\prime}-$ $p_{b}^{\prime}=0$ is fulfilled.

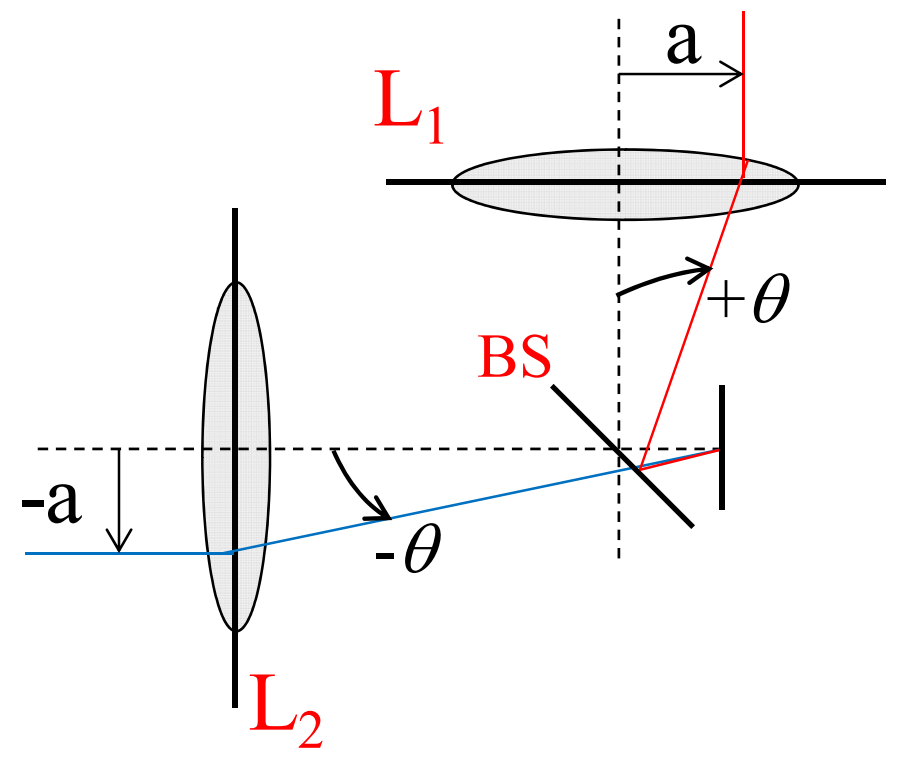

Figure 2.6. The geometrical dynamic picture of the imaging system for achieving the EPR position correlations in measuring the propensity 


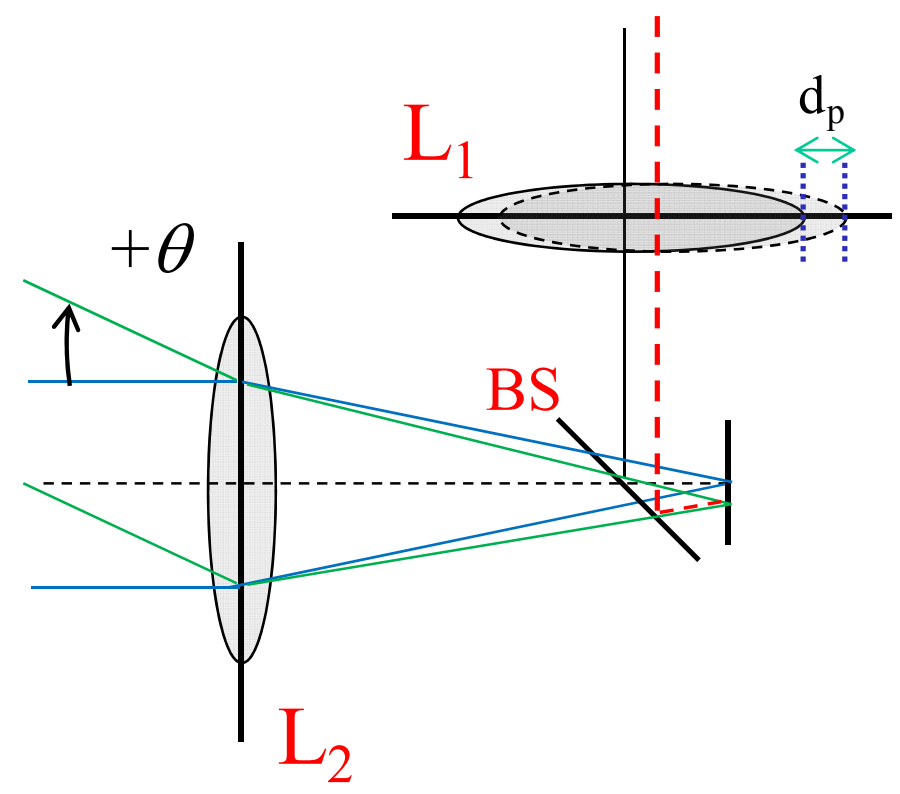

Figure 2.7. The geometrical dynamic picture of the imaging system for achieving the EPR momentum correlations in measuring the propensity.

We denote $X=x_{a}^{\prime}+x_{b}^{\prime}$ and $P=p_{a}^{\prime}-p_{b}^{\prime}$ for the realization of the EPR correlations in the experimental coordinate. The relations of experimental coordinates $x$ and $p$ are formulated from the geometrical dynamic picture of the imaging system that explained before. In real practice, the variances of position $(\Delta X)^{2}$ and momentum $(\Delta P)^{2}$ of the EPR correlations are not zero. These variances can be obtained later through the evaluation of $\mathcal{P}_{b}\left(d_{x}, d_{p}\right)$, which is given by

$$
\begin{aligned}
\mathcal{P}_{b}\left(d_{x}, d_{p}\right) & \propto\left|V_{B}\left(d_{x}, d_{p}\right)\right|^{2} \\
& \propto \mid \exp \left[\frac{1}{4 \sigma_{b}^{2}}\left(d_{x}^{2}-\delta_{p}^{2} \sigma_{b}^{4}-2 i d_{x} \delta_{p} \sigma_{b}^{2}\right)\right] \cos \left(d_{x} D_{p}\right) \\
& +\left.\exp \left[\frac{1}{4 \sigma_{a}^{2}}\left(d_{x}^{2}-\delta_{p}^{2} \sigma_{a}^{4}-2 i d_{x} \delta_{p} \sigma_{a}^{2}\right)\right] \cos \left(a \delta_{p}\right)\right|^{2}
\end{aligned}
$$




$$
\begin{gathered}
\sim\left|\cos \left(d_{x} D_{p}\right)+\cos \left(a \delta_{p}\right)\right|^{2} \\
\sim\left|\cos \left(\left(x_{a}^{\prime}+x_{b}^{\prime}\right) D_{p}\right)+\cos \left(\left(p_{a}^{\prime}-p_{b}^{\prime}\right) a\right)\right|^{2}
\end{gathered}
$$

where we assume a very small displacement or tilt $\left(d_{x} \sim 0\right.$ and $\left.\delta_{p} \sim 0\right)$. The derivation of $\mathcal{P}_{b}\left(d_{x}, d_{p}\right)$ is given in Appendix D.

Through the geometrical dynamic picture of the variables $X=x_{a}^{\prime}+x_{b}^{\prime}$ and $P=p_{a}^{\prime}-p_{b}^{\prime}$ as shown in Fig. 2.6 and Fig. 2.7, we obtain $\mathcal{P}_{b}\left(d_{x}, d_{p}\right)$ in term of $X$ and $P$ given by

$$
\mathcal{P}_{b}\left(d_{x}, d_{p}\right) \sim\left|\cos \left(X D_{p}\right)+\cos (P a)\right|^{2}
$$

Since we can perform direct measurements of the propensity, the system can be used to observe EPR correlations of two independent mirrors in a random manner or to lock mirror 1(2) in the EPR correlation with mirror 2(1). To lock the mirror into EPR correlation one of the mirrors has to be cooled down to isolate it from environmental disturbance and work as the reference mirror. We plot $\mathcal{P}_{b}$ as a function of $d_{x}$ and $\delta_{p}$ at the region of the chessboard near the center spot as shown in Fig. 2.8(a) and a three-dimensional (3D) plot of the center spot as shown in Fig.2.8(b). From Eq. (19), the displacement $d_{x}$ with a magnitude of $\frac{2 \pi}{D_{p}}$ and tilt $\delta_{p}$ with a magnitude of $\frac{2 \pi}{a}$ will cause the system decoherence. We plot the 3 dimensional $\mathcal{P}_{b}\left(d_{x}, d_{p}\right)$ as shown in Fig. 2.8(b) for the center spot with the assumption that $d_{x}$ and $\delta_{p}$ are random variables, simulating the fact that the mirrors 
are independent and subjected to a high-temperature environment. As expected, the random peaks in the $\mathcal{P}_{b}$ plot indicate that the mirrors did exhibit EPR correlations in a random manner. In other words, $\mathcal{P}_{b}$ can be used to extract the EPR correlations of two mirrors or physical systems that associated with them.

In a similar fashion, $\mathcal{P}_{b}$ can be used to lock the mirrors so that they do not fall outside the coherence regions. The existence of other spots around the center spot in the chessboard provides the possibility of quantum jumps in the phase space of the mirrors. To make sure the EPR correlations of mirrors 1 and 2 are quantum correlated, the propensity $\mathcal{P}_{b}\left(d_{x}, d_{p}\right)$ must fall inside the regions in which their variances in position and momentum satisfy the EPR criterion for entanglement [89].

From Eq. 2.20, we calculate the variance for position as

$$
\begin{aligned}
(\Delta X)^{2} & =\left\langle X^{2}\right\rangle-\langle X\rangle^{2} \\
& =\frac{1}{2}\left(\frac{1}{2 D_{p}^{2}}+\frac{\pi^{2}}{3 D_{p}^{2}}\right)
\end{aligned}
$$

and variance for momentum as

$$
\begin{aligned}
(\Delta P)^{2} & =\left\langle P^{2}\right\rangle-\langle P\rangle^{2} \\
& =\frac{1}{2}\left(\frac{1}{2 a^{2}}+\frac{\pi^{2}}{3 a^{2}}\right)
\end{aligned}
$$

The calculation of $(\Delta X)^{2}$ and $(\Delta P)^{2}$ are given in Appendix E. 

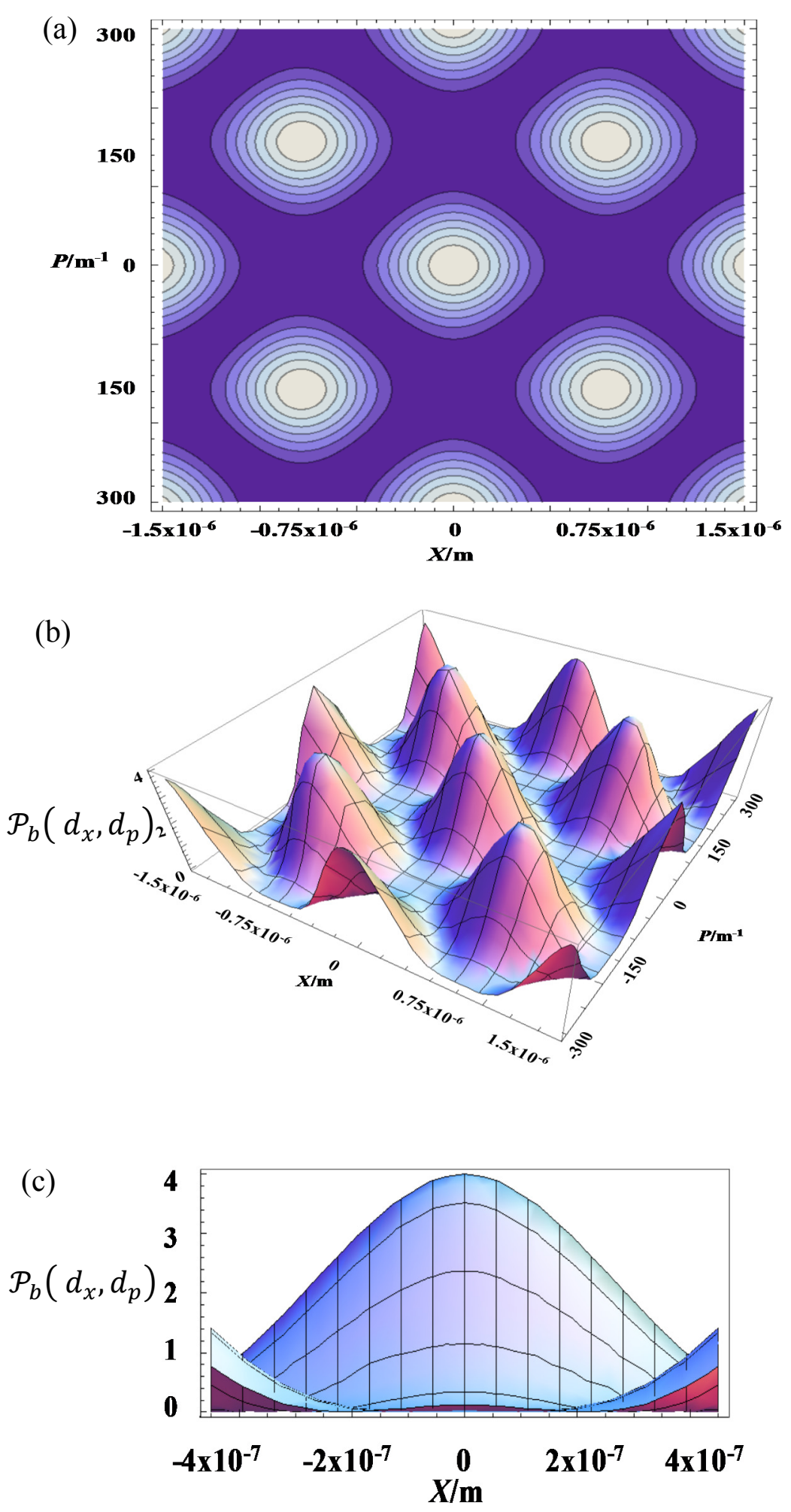

Figure 2.8. (a) The 2D (b) 3D chessboard patterns of the propensity in position $d_{x}$ and momentum $d_{p}(\mathrm{c})$ The 3D plots of the center spot 


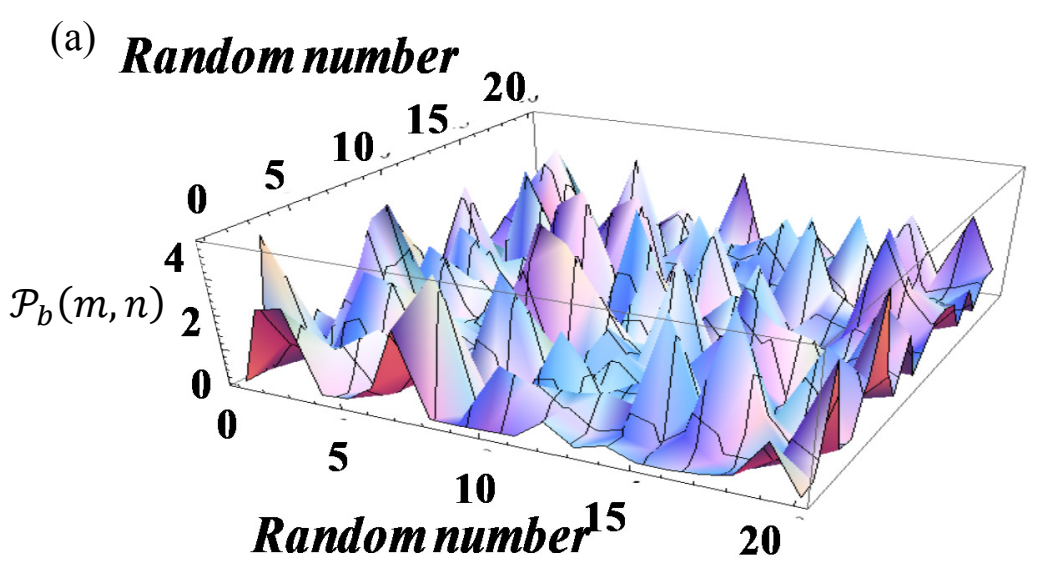

(b)

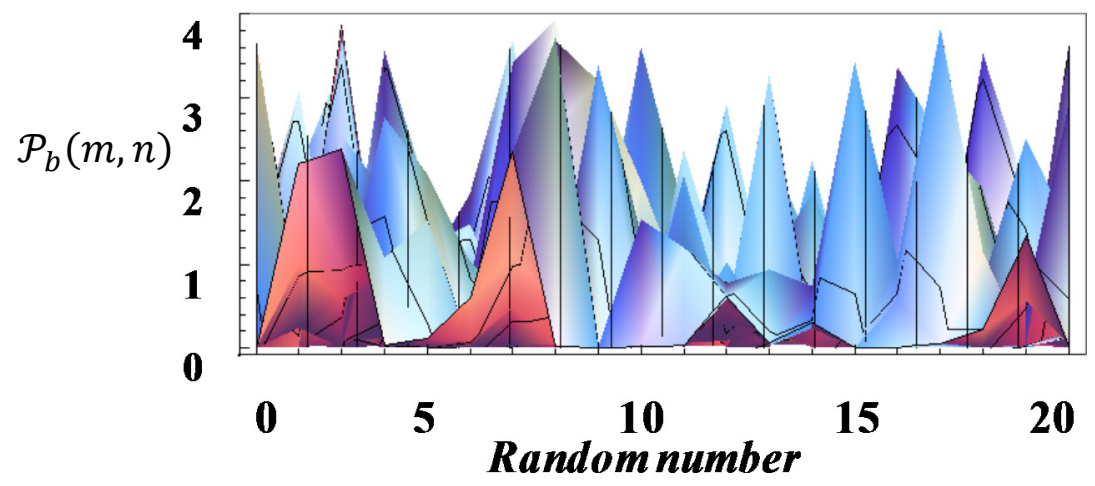

Figure 2.9. (a) $3 \mathrm{D}$ propensity plots of the random-numbers position $d_{x}$ and momentum $d_{p}$. (b) Side view of random-numbers propensity plots.

In addition, we plot the product of $(\Delta X)^{2}(\Delta P)^{2}$ as a function of $D_{p}$ and $a$ as shown in Fig. 2.10. We indicate the set of $\left(D_{p}, a\right)$ that satisfies the criterion for EPR entanglement, $(\Delta X)^{2}(\Delta P)^{2} \leq 1\left[\underline{89}\right.$ ]. For a beam waist of $\sigma_{a}=100 \mu \mathrm{m}$ and wavelength of $\lambda=632 \mathrm{~nm}$, a distance of $a=1 \mathrm{~cm}$, and a focal length of $f=5 \mathrm{~cm}$, we obtain $\Delta X=1.5 \mathrm{~nm}$ and $\Delta P=4 \times 10^{3} / \mathrm{m}$ or $=4 \times 10^{-3} \mathrm{rad}$. The standard quantum limits of measuring displacement and momentum or tilt of a Gaussian 
$\mathrm{TEM}_{00}$ beam that proposed by Delaubert are $d_{S Q L}=\frac{\sigma_{a}}{2 \sqrt{N}}$ and $p_{S Q L}=\frac{1}{\sigma_{a} \sqrt{N}}$, respectively, where $N$ is the number of photons [90]. To achieve $d_{S Q L}=\Delta X$, the required number of photons to arrive on a detector is about $N=10^{9}$. For single photons $N=10^{9}, \Delta X$ of our imaging system is about a $3 \times 10^{4}$ factor better sensitivity than $d_{S Q L}$ while $\triangle P$ has about a 2.4 factor better sensitivity than $p_{S Q L}$.

By using $\mathcal{P}_{b}\left(d_{x}, d_{p}\right)$ as the EPR pointer and the locking region of $\Delta X=$ $1.5 \mathrm{~nm}$ equal to $x_{r m s}=\sqrt{\frac{k_{B} T}{m \omega^{2}}}$ for an object at thermal equilibrium [91]. We can lock or detect two mirrors at room temperature $(300 \mathrm{~K})$ with individual masses of $5 \times$ $10^{-13} \mathrm{~kg}$ at a mechanical frequency of $\sim 10 \mathrm{kHz}$. In real practice, we can cool mirror 2 to the vibration ground state as a reference for locking mirror 1 in a hot environment through $\mathcal{P}_{b}\left(d_{x}, d_{p}\right)$ such that the product of the variances $V\left(x_{h o t}+\right.$ $\left.x_{\text {cold }}\right)$ and $V\left(p_{\text {hot }}-p_{\text {cold }}\right)$ falls within the criterion of EPR entanglement.

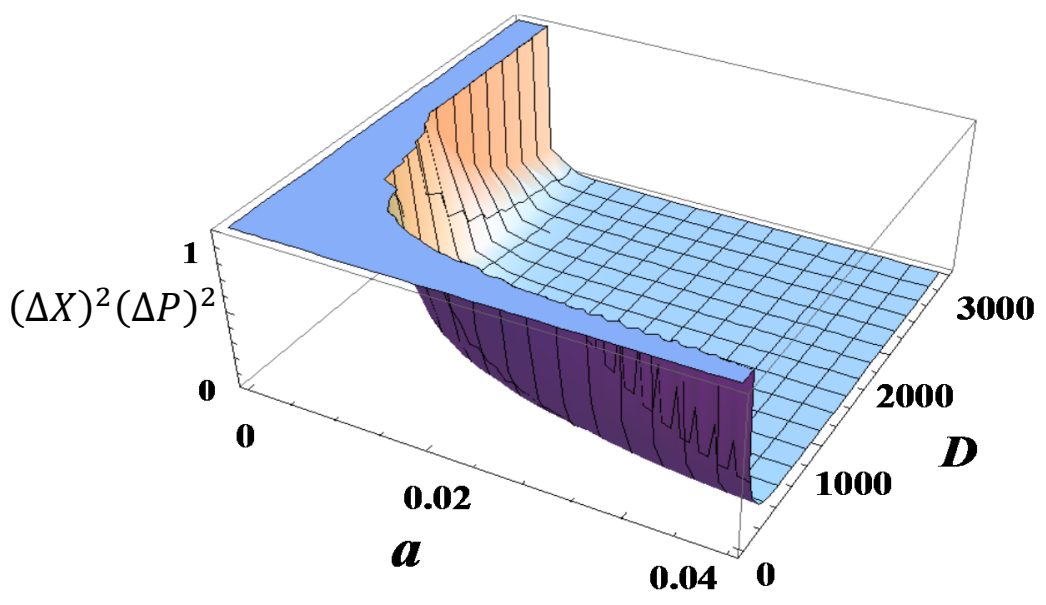

Figure 2.10. The plot of $(\Delta X)^{2}(\Delta P)^{2}$ as a function of $a$ and $D_{p}$, showing the criterion for the EPR entanglement in the region $<1$. 


\subsection{Discrete Propensity}

We can discretize the propensity $\mathcal{P}_{b}\left(d_{x}, d_{p}\right)$ by substituting $m X^{\prime}=d_{x}^{(m)}$ and $n P^{\prime}=$ $\delta_{p}^{(n)}$ where $m, n=0,1,2,3 \ldots, N-1$ are the integers, for $X^{\prime}=\frac{2 \pi}{D_{p}} \quad$ and $P^{\prime}=\frac{2 \pi}{a}$ respectively. Now the propensity $\mathcal{P}_{b}\left(d_{x}, d_{p}\right)$ can be written in a function of $m$ and $n$ as

$$
\mathcal{P}_{b}\left(d_{x}, d_{p}\right) \rightarrow \mathcal{P}_{b}(m, n) \sim|\cos (m \pi)+\cos (n \pi)|^{2}
$$

In Fig. 2.8(a), $X^{\prime}$ is the distance spacing between three spots (left, center, and right), and $P^{\prime}$ is the distance spacing between the bottom, center, and top spots. The product of $X^{\prime} P^{\prime}=\frac{2 \pi}{a} \frac{2 \pi}{D_{p}}=\frac{(2 \pi)^{2}}{A}=\alpha$ corresponds to the sub-Planck area in phase space that covers one EPR correlation spot in the propensity of $\mathcal{P}_{b}$. This area $\alpha$ can be treated as the phase-space action and the sensitivity of a quantum system to perturbations $[\underline{25}, \underline{64}]$. Since we have many of these correlation spots, the perturbation from the environment on one of the mirrors may not destroy the quantum correlations between the mirrors but instead jump into other spots. For each set of $(m, n)$, we have a pair of EPR correlations such that the variances of the position $\left(\Delta X^{(m)}\right)^{2}=\left(\Delta\left[x_{a}^{(m)}+x_{b}^{(m)}\right]\right)^{2}$ and the momentum $\left(\Delta P^{(n)}\right)^{2}=$ $\left(\Delta\left[p_{a}^{(n)}-p_{b}^{(n)}\right]\right)^{2}$ fulfill the EPR entanglement. 
We can form the finite-dimensional Hilbert space of the propensity $\mathcal{P}_{b}$ by introducing base of $N$ orthogonal normalized vectors $\left|d_{x}^{(m)}\right\rangle$ and $\left|d_{p}^{(n)}\right\rangle$. These bases pair are discrete Fourier transforms of each other such that $[\underline{92}, \underline{93}]$

$$
\left|d_{x}^{(m)}\right\rangle=\frac{1}{N} \sum_{n=0}^{N-1} \exp \left(-i X^{\prime} P^{\prime} m n\right)\left|d_{p}^{(n)}\right\rangle
$$

and

$$
\left|d_{p}^{(n)}\right\rangle=\frac{1}{N} \sum_{n=0}^{N-1} \exp \left(i X^{\prime} P^{\prime} m n\right)\left|d_{x}^{(m)}\right\rangle
$$

We can convert $X^{\prime} P^{\prime}=\frac{2 \pi}{N}$ by dividing the area $A$ (classical action) by $2 \pi$ to obtain $=$ $\frac{A}{2 \pi}$. Then the discrete form of the propensity can be established as

$$
\mathcal{P}_{b}(m, n)=\sum_{k, l} \mathcal{W}_{\odot_{2}}(k-m, l-n) \mathcal{W}_{\odot_{1}}(k, l)
$$

for exploring the macroscopic mechanical correlations. The discrete phase-space spot in $\mathcal{P}_{b}(m, n)$ can be used to explore environmental perturbed quantum jumps of the EPR correlations as was predicted in stochastic random telegraph jumps between two individual spins [94]. 


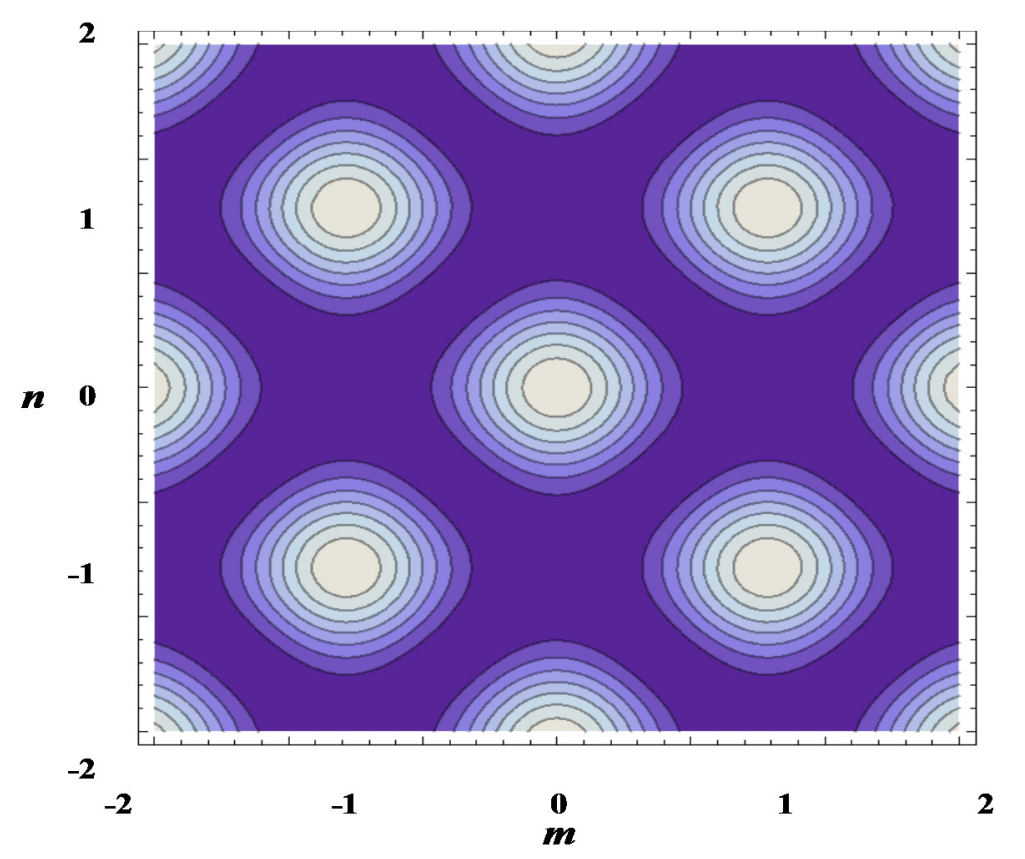

Figure 2.11. Chessboard pattern of discretized propensity $\mathcal{P}_{b}(m, n) \sim \mid \cos (m \pi)+$ $\left.\cos (n \pi)\right|^{2}$ in random number $m$ and $n$.

\subsection{Discussions}

The spatial compass states can be displaced and tilted by classical and quantum spatial fluctuations of two independent mirrors. After these mirrors, the spatial compass states were directed into an imaging system which performs projection measurements on them with a single-photon detector. By detecting single photons, we created the entanglement of these two mirrors in terms of the position states $\left|x_{1}, x_{2}, x_{3}, \ldots\right\rangle_{a}+\left|x_{1^{\prime}}, x_{2^{\prime}}, x_{3^{\prime}}, \ldots\right\rangle_{b} \quad$ or momentum states $\left|p, p_{2}, p_{3}, \ldots\right\rangle_{a}+$ $\left|p_{1^{\prime}}, p_{2^{\prime}}, p_{3^{\prime}}, \ldots\right\rangle_{b}$ as we described in the geometrical dynamic process of two mirrors. Here, the product states of the position (translation) and momentum (tilt or angle) of 
these mirrors are attributed to the chessboard pattern of the spatial compass states, which are then realized through the discrete properties of the propensity.

The chessboard pattern of the spatial compass states reflects the spatial properties of a mirror. We can treat a mirror as if it is composed of $\mathrm{N}$ quantum mirrors with quantized position $\left(x_{i}, i=1,2,3, \ldots N\right)$ and momentum $\left(p_{i}, i=\right.$ $1,2,3, \ldots N)$. We then performed a direct projection measurement on the chessboard pattern of two spatial compass states via the propensity, i.e. $\mathcal{P}_{B} \propto \iint d x d p W_{\odot_{2}}(x-$ $\left.x_{0}, p-p_{0}\right) W_{\odot_{1}}(x, p)$, The spatial EPR correlations of these mirrors were realized through the dynamical transformation, i.e., $\left(x_{b}=x-x_{0}=x_{a}-x_{0}, p_{b}=p+p_{0}=\right.$ $\left.p_{a}+p_{0}\right)$, in the process of measuring the propensity.

We could also extract the EPR correlations of these mirrors in terms of position and momentum (tilt) coordinates. If two independent mirrors were subjected to random spatial fluctuations, the appearance of the random maximum peak of $\mathcal{P}_{B}$ is the indication that the two mirrors were EPR correlated. In addition, the imaging system can lock these mirrors in the EPR correlations through the interference of the compass states. To justify that these macroscopic mechanical correlations are quantum in nature, the criteria for EPR entanglement of the two mirrors were then obtained through the sub-Planck structure of the propensity.

The proposed optical phase-space imaging system based on spatial compass states and single photons can be used for observing macroscopic mechanical quantum correlations of two mirrors in terms of position and momentum (angle or tilt) 
coordinates. The realistic motions of measuring the propensity in the imaging system can extract the EPR correlations of two mirrors. Since the propensity $\mathcal{P}_{b}$ can be discretized and formulated in finite-dimensional Hilbert space, direct detection of the discrete propensity is a quantum measurement that observes the quantum jumps of spatial EPR correlations of two mirrors and, hence, enables discrete phase-space quantum computing and information processing [95-97]. 



\section{Chapter 3}

\section{Intrinsic Quantum Correlation of Weak Coherent States ${ }^{2}$}

\subsection{Overview}

In this chapter, we demonstrated a proof of principle experiment in utilizing intrinsic quantum correlations of weak coherent states for quantum communication.

First of all we present a brief review on the development of the quantum communication. We highlight the challenges in the implementation of both discrete and continuous quantum variables based quantum communication. Followed by a discussion on the various quantum key generation schemes using weak coherent states.

In next section, we will discuss on how to implement 4 bipartite correlation functions between two distant observers by using two orthogonal light fields with a balanced detection scheme. After that we go through briefly on detection apparatus that utilized in our experiment such as photo detector and transimpedance amplifier,

\footnotetext{
2 The material contained in this chapter was previously published in Physical Review A.
} 
electrical spectrum analyzer, digital oscilloscope and lock-in amplifier. Then, we will describe in detail about the balanced homodyne detector that employed as detection scheme for the weak coherent states. Balanced homodyne detector is crucial in obtaining bipartite correlations of weak coherent states.

In the following section, we will present the experiment setups to generate four types of bipartite correlations function between two distant observers by two weak coherent states. In addition, we will walk through the derivation in obtaining all four bipartite correlations functions $\Psi^{-}, \Psi^{+}, \phi^{-}$and $\phi^{+}$. We reveal that information of the interference signal will be protected by the large quantum phase noise fluctuation of weak coherent states.

In the experiment results section, first we present both two weak coherent states interference that obtained by each observers by using balanced homodyne detector. Then we obtain the bipartite correlations between of two observers by multiplying the interference signal of both observers. Also, experimental measurements of all four bipartite correlations functions are obtained in this section. As for the use of quantum communication between two distant observers, we establish the bipartite correlations and demonstrate bits correlations measurement of each bipartite correlation at detectors A and B by using lock-in amplifier.

\subsection{Introduction}

Quantum entanglement and superposition are foundations for the emerging field of quantum communication and information processing. These two fundamental 
features of quantum mechanics have made quantum key distribution unconditionally secure compared to communication based on classical key distribution [15].

Currently, implementation of an optical quantum communication is mainly based on discrete and continuous quantum variables. They are usually generated through optical nonlinear interaction processes via second and third order susceptibilities $[\underline{58}, \underline{98}]$ of the media. Discrete-variable qubit based implementations using polarization and time-bin entanglement are difficult to obtain unconditional security $[99,100]$. Sustained optical data-rate are limited because of post-selection technique with low probability of success in a low efficiency single photon detector at telecom-band [101]. In addition, an entanglement-based key generation such as Ekert's protocol is hard to implement in a real-world optical fiber network because bipartite correlations of entangled photon-pairs are sensitive to loss and decoherence [13].

Continuous-variable implementations using quadrature entanglement and polarization squeezing could have achieved high efficiency and high optical data-rate because of available high speed and efficient homodyne detection techniques [102, 103]. However, the quality of quadrature entanglement is very sensitive to loss, which makes it difficult to implement entanglement based quantum protocols over long distance. Continuous-variable protocols, that do not rely on entanglement, for instance, coherent-state based quantum communication, are perfect for long distance optical communication [104, $\underline{105}]$. 
Quantum key distribution using weak coherent states, such as coherent state differential phase-shift quantum key distribution (DPS-QKD) and decoy-states Bennett-Brassard 1984 (BB84) protocols, have been proven to be unconditionally secure against a photon-number splitting attacks (PNS) $[\underline{14}, \underline{29}, \underline{106}]$. The DPS-QKD uses intrinsic correlations between the relative phase shifts $\{0, \pi\}$ of two consecutive pulses to achieve unconditional security between two parties by constructing equivalent states for the entanglement-based protocol [106]. The decoy state quantum key distribution uses intrinsic correlations between the relative mean photon numbers of two sets of weak coherent states to detect the PNS attack in BB84 protocol [107]. Meanwhile, an alternatives Y00 protocol uses intrinsic correlations between phase and mean photon number fluctuations of weak coherent states to provide cryptographic service of data encryption between two parties. Intrinsic quantum correlations of coherent states can be prepared, measured, and shared between two parties for quantum cryptography [108]. We propose here a scheme based on weak coherent states for generating intrinsic bipartite correlations as a supplemental resource to the existence protocols such as coherent state DPS-QKD and decoy state BB84 protocol.

\subsection{Weak Coherent states Bipartite Correlations}

A proof-of-principle experimental setup to demonstrate the bipartite correlations of two weak coherent states is shown in Fig.3.1. A continuous wave laser at telecom band wavelength $(1534 \mathrm{~nm})$ is used to provide two orthogonally polarized weak coherent states $|\alpha\rangle$ and $\left|\beta_{L O}\right\rangle$. We use a piezoelectric transducer (PZT) to ramp the 
phase $\phi_{\beta}$ of the $\left|\beta_{L O}\right\rangle$ weak coherent states which the weak local oscillator (LO) beam. We use a $50 / 50$ beam splitter to optically mix the vertically polarized weak coherent state $|\alpha\rangle$ and horizontally polarized weak coherent state $\left|\beta_{L O}\right\rangle$. The beam 1 from the output port 1 of the beam splitter is a superposition of the vertically and horizontally polarized weak coherent states $|\alpha\rangle$ and $\left|\beta_{L O}\right\rangle$, similarly for beam 2 from output port 2 of the beam splitter. We couple beam 2 into the $10 \mathrm{~km}$ long transmission fiber. A quarter-wave plate and a half-wave plate are used at the output of the transmission fiber to compensate the birefringence. The transformation matrix for quarter-wave plate and half-wave plate that were used in our experiment are given as

$$
\mathbf{T}_{\mathrm{QWP} \pm 45^{\circ}}=\frac{1}{\sqrt{2}}\left[\begin{array}{cc}
1 & \mp i \\
\mp i & 1
\end{array}\right]
$$

and

$$
\mathbf{T}_{\mathrm{HWP}^{\circ}}=\left[\begin{array}{cc}
-i & 0 \\
0 & i
\end{array}\right] .
$$

The input field operators $\hat{a}$ and $\hat{b}$ at the beam splitter are the annihilation operators for the weak coherent state $|\alpha\rangle=\left|\alpha e^{i \phi_{\alpha}}\right\rangle$ and $\left|\beta_{L O}\right\rangle=\left|\beta_{L O} e^{i \phi_{\beta}}\right\rangle$, respectively. The the output field operators in beam 1 at the beam splitter splitter are

$$
\hat{c}_{1}=\frac{1}{\sqrt{2}}(\hat{a} \mathbf{x}+i \hat{b} \mathbf{y})=\frac{1}{\sqrt{2}}\left(\hat{a}\left[\begin{array}{l}
\mathbf{1} \\
\mathbf{0}
\end{array}\right]+i \hat{b}\left[\begin{array}{l}
\mathbf{0} \\
\mathbf{1}
\end{array}\right]\right)
$$

and 


$$
\hat{d}_{1}=\frac{1}{\sqrt{2}}(i \hat{a} \mathbf{x}+\hat{b} \mathbf{y})=\frac{1}{\sqrt{2}}\left(i \hat{a}\left[\begin{array}{l}
\mathbf{1} \\
\mathbf{0}
\end{array}\right]+\hat{b}\left[\begin{array}{l}
\mathbf{0} \\
\mathbf{1}
\end{array}\right]\right)
$$

in beam 2. Where, $\mathbf{x}$ and $\mathbf{y}$ indicate horizontal and vertical axis in conjunction to the polarization of the weak coherent states.

We are able to establish four types of bipartite correlations with this experiment setup by using linear phase shifters on either beam 1 or beam 2 . In the context of quantum communication, we keep setup for beam 2 unchanged and vary the linear phase shifter of beam 1 locally to change the coherent state bipartite correlation function shared between two observers.

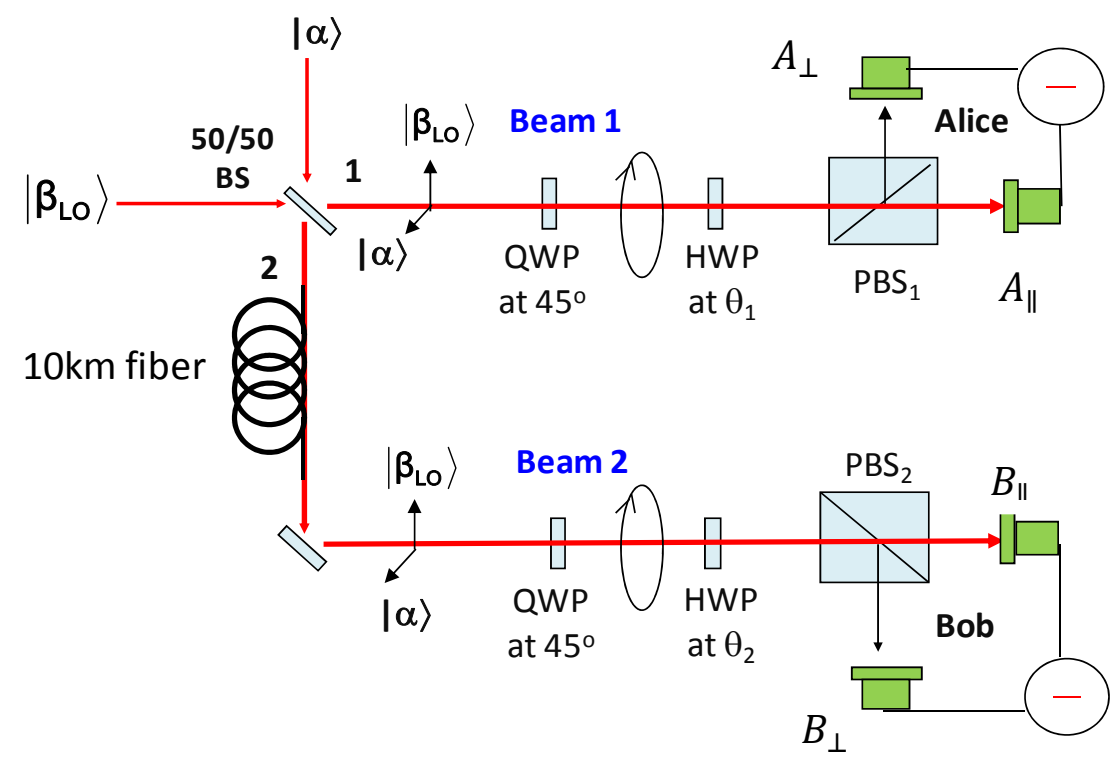

Figure 3.1. Experiment setup for demonstration of Correlation function $\Psi^{-}=$ $-\cos 2\left(\theta_{1}-\theta_{2}\right)$ with weak coherent states. BS (beam splitter); PBS (polarization beam splitter); HWP and QWP (half- and quarter-wave plates); D (photodiode). 


\subsubsection{Correlation function $\boldsymbol{\Psi}^{-}=-\cos 2\left(\boldsymbol{\theta}_{1}-\boldsymbol{\theta}_{2}\right)$}

A quarter-wave plate was inserted at $45^{\circ}$ into beam 1 to transform the linearly polarized states to circularly polarized states. The quarter wave plate transforms the field operators $\hat{c}_{1}$ into $\hat{c}_{1}^{\prime}$ as,

$$
\begin{aligned}
\hat{c}_{1} \rightarrow \hat{c}_{1}^{\prime}= & \frac{1}{\sqrt{2}}\left[\begin{array}{cc}
1 & -i \\
-i & 1
\end{array}\right] \frac{1}{\sqrt{2}}\left(\hat{a}\left[\begin{array}{l}
\mathbf{1} \\
\mathbf{0}
\end{array}\right]+i \hat{b}\left[\begin{array}{l}
\mathbf{0} \\
\mathbf{1}
\end{array}\right]\right) \\
& =\frac{1}{2}\{(\hat{a}+\hat{b}) \mathbf{x}+i(\hat{b}-\hat{a}) \mathbf{y}\} .
\end{aligned}
$$

Field operator $\hat{c}_{1}^{\prime}$ is a homogeneous superposition of left circularly polarized and right circularly polarized weak coherent states. Similarly, field operators $\hat{d}_{1}$ in beam 2 will be transformed by a QWP at $45^{\circ}$ as,

$$
\begin{aligned}
\hat{d}_{1} \rightarrow \hat{d}_{1}^{\prime} & =\frac{1}{\sqrt{2}}\left[\begin{array}{cc}
1 & -i \\
-i & 1
\end{array}\right] \frac{1}{\sqrt{2}}\left(i \hat{a}\left[\begin{array}{l}
\mathbf{1} \\
\mathbf{0}
\end{array}\right]+\hat{b}\left[\begin{array}{l}
\mathbf{0} \\
\mathbf{1}
\end{array}\right]\right) \\
& =\frac{1}{2}\{i(\hat{a}-\hat{b}) \mathbf{x}+(\hat{a}+\hat{b}) \mathbf{y}\} .
\end{aligned}
$$

A polarization analyzer that consists of a half-wave plate (HWP1) and a polarization beam splitter cube (PBS1) was inserted in beam 1 . The unit vector that associated with the polarization analyzer is given as:

$$
\hat{e}_{1}=\cos \theta_{1} \mathbf{x}+\sin \theta_{1} \mathbf{y}
$$

Now the field operator for the transmitted component of PBS1 can be written as 


$$
\begin{aligned}
\hat{c}_{1}^{\prime} \rightarrow \hat{c}_{1}^{\prime \prime}=\hat{c}_{1}^{\prime} \cdot \hat{e}_{1} & =\frac{1}{2}\{(\hat{a}+\hat{b}) \mathbf{x}+i(\hat{b}-\hat{a}) \mathbf{y}\} \cdot\left(\cos \theta_{1} \mathbf{x}+\sin \theta_{1} \mathbf{y}\right) . \\
\hat{c}_{1}^{\prime \prime} & =\frac{1}{2}\left[\hat{a}\left(\cos \theta_{1}-i \sin \theta_{1}\right)+\hat{b}\left(\cos \theta_{1}+i \sin \theta_{1}\right)\right] .
\end{aligned}
$$

Similarly for beam 2, field operator for the transmitted component at PBS2 is given as

$$
\begin{gathered}
\hat{d}_{2}^{\prime} \rightarrow \hat{d}_{2}^{\prime \prime}=\hat{d}_{2}^{\prime} \cdot \hat{e}_{2} \\
\hat{d}_{2}^{\prime \prime}=\frac{1}{2}\left[\hat{a}\left(i \cos \theta_{2}+\sin \theta_{2}\right)+\hat{b}\left(\sin \theta_{2}-i \cos \theta_{2}\right)\right] .
\end{gathered}
$$

Where $\hat{e}_{2}=\cos \theta_{2} \mathbf{x}+\sin \theta_{2} \mathbf{y}$ and $\theta_{2}$ is projection angle of the polarization analyzer at beam 2. The photon number operator of the transmitted component at PBS 1 can be written as

$$
\begin{aligned}
\hat{c}_{1}^{\prime \prime \dagger} \hat{c}_{1}^{\prime \prime}= & \frac{1}{2}\left[\hat{a}^{\dagger}\left(\cos \theta_{1}+i \sin \theta_{1}\right)+\hat{b}^{\dagger}\left(\cos \theta_{1}-i \sin \theta_{1}\right)\right] \\
& \times \frac{1}{2}\left[\hat{a}\left(\cos \theta_{1}-i \sin \theta_{1}\right)+\hat{b}\left(\cos \theta_{1}+i \sin \theta_{1}\right)\right] \\
= & \frac{1}{4}\left[\hat{a}^{\dagger} \hat{a}+\hat{b}^{\dagger} \hat{b}+\hat{a}^{\dagger} \hat{b} e^{i 2 \theta_{1}}+\hat{b}^{\dagger} \hat{a} e^{-i 2 \theta_{1}}\right] .
\end{aligned}
$$

Photon number operator of the transmitted component at PBS 2 becomes

$$
\begin{aligned}
\hat{d}_{2}^{\prime \prime}{ }^{\dagger} \hat{d}_{2}^{\prime \prime}=\frac{1}{2}[\hat{a}( & \left.\left.-i \cos \theta_{2}+\sin \theta_{2}\right)+\hat{b}\left(\sin \theta_{2}+i \cos \theta_{2}\right)\right] \\
& \times \frac{1}{2}\left[\hat{a}\left(i \cos \theta_{2}+\sin \theta_{2}\right)+\hat{b}\left(\sin \theta_{2}-i \cos \theta_{2}\right)\right] \\
= & \frac{1}{4}\left[\hat{a}^{\dagger} \hat{a}+\hat{b}^{\dagger} \hat{b}-\hat{a}^{\dagger} \hat{b} e^{i 2 \theta_{2}}-\hat{b}^{\dagger} \hat{a} e^{-i 2 \theta_{2}}\right] .
\end{aligned}
$$


Considering the weak coherent state $|\alpha\rangle=\left|\alpha e^{i \phi_{\alpha}}\right\rangle$ and weak LO field $\left|\beta_{L O}\right\rangle=\left|\beta e^{i \phi_{\beta}}\right\rangle$, the beat intensities of transmitted component at PBS1 and PBS2 that were measured by detectors $A_{\|}$and $B_{\|}$can be expressed as

$$
\begin{aligned}
& I_{A_{\|}}\left(\theta_{1 \|}\right)=\left\langle\alpha, \beta\left|\hat{c}_{1}^{\prime \prime \dagger} \hat{c}_{1}^{\prime \prime}\right| \alpha, \beta\right\rangle \\
= & \eta_{A}\left\{|\alpha|^{2}+|\beta|^{2}+|\alpha||\beta| \cos \left(2 \theta_{1}+\phi_{\beta}-\phi_{\alpha}\right)\right\}
\end{aligned}
$$

and

$$
\begin{aligned}
I_{B_{\|}}\left(\theta_{2 \|}\right) & =\left\langle\alpha, \beta\left|\hat{d}_{2}^{\prime \prime}{ }^{\dagger} \hat{d}_{2}^{\prime \prime}\right| \alpha, \beta\right\rangle \\
& =\eta_{B}\left\{|\alpha|^{2}+|\beta|^{2}-|\alpha||\beta| \cos \left(2 \theta_{2}+\phi_{\beta}-\phi_{\alpha}\right)\right\} .
\end{aligned}
$$

$\eta_{A}$ and $\eta_{B}$ are the photocurrent conversion efficiency (Optical power $\rightarrow$ electric current) for detection electronics of $\operatorname{detector} \mathrm{A}$ and B. The first two terms $|\alpha|^{2}+$ $|\beta|^{2}$ are intensities of the two weak coherent states and the last term $|\alpha||\beta| \cos \left(2 \theta_{1}+\phi_{\beta}-\phi_{\alpha}\right)$ is the interference term consisting of polarization angle $\theta_{1}$, the phases of $\operatorname{LO}\left(\phi_{\beta}\right)$, and the weak coherent state $\left(\phi_{\alpha}\right)$. The beat intensity $I_{A_{\|}}\left(\theta_{1}\right)$ is anticorrelated to $I_{B_{\|}}\left(\theta_{2}\right)$ because of the $\pi$-phase shift induced by the $50-50$ beam splitter.

The reflected component measured of PBS1 at $\theta_{1 \perp}=\theta_{1 \|}+90^{\circ}$ by detector $A_{\perp}$ is given as 


$$
\begin{aligned}
& I_{A_{\perp}}\left(\theta_{1 \perp}\right)=\left\langle\alpha, \beta\left|\hat{c}_{1}^{\prime \prime} \hat{c}_{1}^{\prime \prime}\right| \alpha, \beta\right\rangle \\
& =\eta_{A}\left\{|\alpha|^{2}+|\beta|^{2}+|\alpha||\beta| \cos \left(2\left(\theta_{1}+90^{\circ}\right)+\phi_{\beta}-\phi_{\alpha}\right)\right\} \\
& \quad=\eta_{A}\left\{|\alpha|^{2}+|\beta|^{2}-|\alpha||\beta| \cos \left(2 \theta_{1}+\phi_{\beta}-\phi_{\alpha}\right)\right\} .
\end{aligned}
$$

While the reflected component that measured of PBS2 at $\theta_{2 \perp}=\theta_{2 \|}+90^{\circ}$ detector $B_{\perp}$ is given as

$$
\begin{aligned}
I_{B_{\perp}}\left(\theta_{2 \perp}\right)=\left\langle\alpha, \beta\left|\hat{d}_{2}^{\prime \prime} \hat{d}_{2}^{\prime \prime}\right| \alpha, \beta\right\rangle & \\
& =\eta_{B}\left\{|\alpha|^{2}+|\beta|^{2}-|\alpha||\beta| \cos \left(2\left(\theta_{2}+90\right)+\phi_{\beta}-\phi_{\alpha}\right)\right\} \\
& =\eta_{B}\left\{|\alpha|^{2}+|\beta|^{2}+|\alpha||\beta| \cos \left(2 \theta_{2}+\phi_{\beta}-\phi_{\alpha}\right)\right\}
\end{aligned}
$$

The balanced homodyne detectors A and B measured beat intensities that were,

$$
\begin{aligned}
& A_{B H D}=I_{A_{\|}}\left(\theta_{1 \|}\right)-I_{A_{\perp}}\left(\theta_{1 \perp}\right)=2 \eta_{A}|\alpha||\beta| \cos \left(2 \theta_{1}+\phi_{\beta}-\phi_{\alpha}\right), \\
& B_{B H D}=I_{B_{\|}}\left(\theta_{1 \|}\right)-I_{B_{\perp}}\left(\theta_{1 \perp}\right)=-2 \eta_{A}|\alpha||\beta| \cos \left(2 \theta_{2}+\phi_{\beta}-\phi_{\alpha}\right) .
\end{aligned}
$$

The individual intensities of the two coherent states $|\alpha|^{2}+|\beta|^{2}$ were subtracted. Information $2 \theta_{1}+\phi_{\beta}$ and $2 \theta_{2}+\phi_{\beta}$ are protected by quantum phase noise $\phi_{\alpha}$ with phase fluctuation of $\Delta \phi_{\alpha}=\frac{1}{\Delta n}$. The low mean photon number fluctuation $\Delta n$ of weak coherent state naturally induces large phase fluctuation.

The balanced homodyne beat intensities measured by detectors A and B were multiplied to obtain 


$$
\begin{aligned}
A_{B H D} \cdot B_{B H D} & =2 \eta_{A}|\alpha||\beta| \cos \left(2 \theta_{1}+\phi_{\beta}-\phi_{\alpha}\right) \cdot-2 \eta_{B}|\alpha||\beta| \cos \left(2 \theta_{2}+\phi_{\beta}-\phi_{\alpha}\right) \\
= & -4 \eta_{A} \eta_{B}\left\{|\alpha|^{2}|\beta|^{2} \cos 2\left(\theta_{1}-\theta_{2}\right)+\cos 2\left(\theta_{2}+\theta_{1}+\phi_{\beta}-\phi_{\alpha}\right)\right\} .
\end{aligned}
$$

The multiplied balanced-homodyne beat intensities $A_{B H D} \cdot B_{B H D}$ did not provide bipartite correlation functions directly. However, by taking the mean value of these multiplied beat intensities, the last term is averaged to zero due to the slowly varying local oscillator phase $\phi_{\beta}$ from $\{0 \rightarrow 2 \pi\}$.

Hence, we obtained the expectation value of two detectors or coherent state bipartite correlation function as:

$$
\begin{aligned}
\left\langle A_{B H D} \cdot B_{B H D}\right\rangle & =-4 \eta_{A} \eta_{B}|\alpha|^{2}|\beta|^{2} \cos 2\left(\theta_{1}-\theta_{2}\right) \\
& \propto-\cos 2\left(\theta_{1}-\theta_{2}\right) .
\end{aligned}
$$

Note that, the quantum phase noise $\phi_{\alpha}$ cannot randomly provide phase shift from $\{0 \rightarrow 2 \pi\}$. Therefore, bipartite correlation function $-\cos 2\left(\theta_{1}-\theta_{2}\right)$ is protected by the term $\cos 2\left(\theta_{2}+\theta_{1}+\phi_{\beta}-\phi_{\alpha}\right)$, which was averaged to zero. In practice, the product of mean photon numbers $|\alpha|^{2}|\beta|^{2}$ can be obtained by setting the correlation function to its maximum obtainable value, that is, $\theta_{1}=\theta_{2}$. The raw data of the multiplied beat intensities are then normalized with the product of $4 \eta_{A} \eta_{B}|\alpha|^{2}|\beta|^{2} \cos 2\left(\theta_{1}-\theta_{2}\right)$ to obtain correlation function $-\cos 2\left(\theta_{1}-\theta_{2}\right)$. 


\subsubsection{Correlation function $\Psi^{+}=\cos 2\left(\theta_{1}-\theta_{2}\right)$}

In order to prepare the second correlation function $\Psi^{+}=\cos 2\left(\theta_{1}-\theta_{2}\right)$, we insert a HWP at $0^{\circ}$ into beam 1 as shown in Fig.3.2. The field operators $\hat{c}_{1}$ before the QWP will be transformed into,

$$
\begin{aligned}
\hat{c}_{1} \rightarrow \hat{c}_{H W P} & =\left[\begin{array}{cc}
-i & 0 \\
0 & i
\end{array}\right] \frac{1}{\sqrt{2}}\left(\hat{a}\left[\begin{array}{l}
\mathbf{1} \\
\mathbf{0}
\end{array}\right]+i \hat{b}\left[\begin{array}{l}
\mathbf{0} \\
\mathbf{1}
\end{array}\right]\right) \\
& =\frac{1}{\sqrt{2}}\left(\hat{a}\left[\begin{array}{c}
-\boldsymbol{i} \\
\mathbf{0}
\end{array}\right]+i \hat{b}\left[\begin{array}{c}
\mathbf{0} \\
\mathbf{- 1}
\end{array}\right]\right) .
\end{aligned}
$$

After the QWP the field operators $\hat{c}_{1}$ will be transformed into,

$$
\begin{aligned}
\hat{c}_{H W P} \rightarrow \hat{c}_{1}^{\prime}= & \frac{1}{\sqrt{2}}\left[\begin{array}{cc}
1 & -i \\
-i & 1
\end{array}\right] \frac{1}{\sqrt{2}}\left(\hat{a}\left[\begin{array}{c}
-\boldsymbol{i} \\
\mathbf{0}
\end{array}\right]+i \hat{b}\left[\begin{array}{c}
\mathbf{0} \\
-\mathbf{1}
\end{array}\right]\right) \\
& =\frac{1}{2}\{(-i \hat{a}+\widehat{\imath} \hat{b}) \mathbf{x}+(-\hat{b}-\hat{a}) \mathbf{y}\} .
\end{aligned}
$$

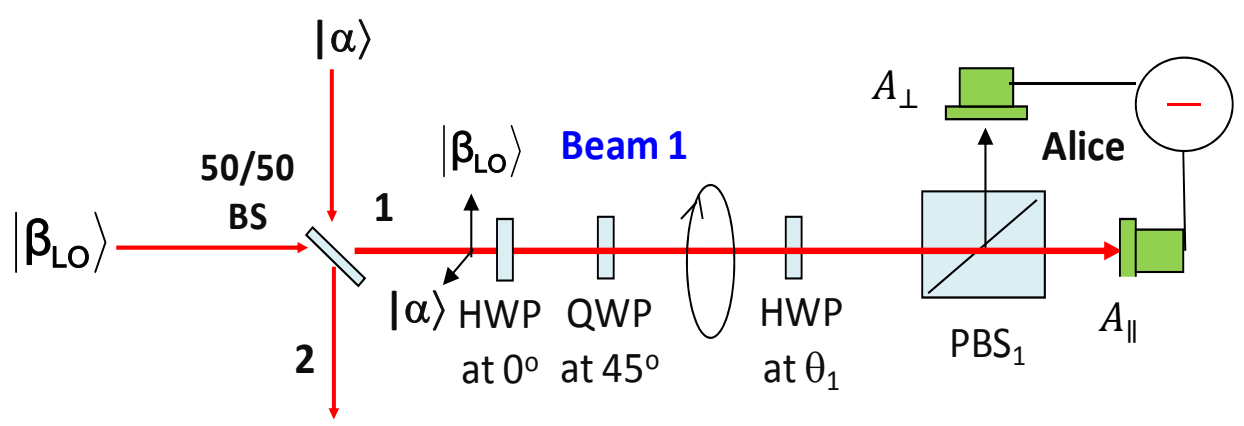

Figure 3.2. Experiment setup used for demonstration of Correlation function $\Psi^{+}=$ $\cos 2\left(\theta_{1}-\theta_{2}\right)$

After the polarization analyzer, the field operator for the transmitted component of PBS1 is given as: 


$$
\begin{aligned}
\hat{c}_{1}^{\prime} \rightarrow \hat{c}_{1}^{\prime \prime}=\hat{c}_{1}^{\prime} \cdot \hat{e}_{1}= & \frac{1}{2}\{(-i \hat{a}+\widehat{\imath b}) \mathbf{x}+(-\hat{b}-\hat{a}) \mathbf{y}\} \cdot\left(\cos \theta_{1} \mathbf{x}+\sin \theta_{1} \mathbf{y}\right) \\
\hat{c}_{1}^{\prime \prime} & =\frac{1}{2}\left[\hat{a}\left(-i \cos \theta_{1}-\sin \theta_{1}\right)+\hat{b}\left(i \cos \theta_{1}-\sin \theta_{1}\right)\right] .
\end{aligned}
$$

The photon number operator of the transmitted component of at PBS 1 can be written as

$$
\begin{aligned}
\hat{c}_{1}^{\prime \prime} \hat{c}_{1}^{\prime \prime}=\frac{1}{2}\left[\hat{a}^{\dagger}\left(\cos \theta_{1}+i \sin \theta_{1}\right)+\hat{b}^{\dagger}\left(\cos \theta_{1}-i \sin \theta_{1}\right)\right] \\
\quad \times \frac{1}{2}\left[\hat{a}\left(\cos \theta_{1}-i \sin \theta_{1}\right)+\hat{b}\left(\cos \theta_{1}+i \sin \theta_{1}\right)\right] \\
=\frac{1}{4}\left[\hat{a}^{\dagger} \hat{a}+\hat{b}^{\dagger} \hat{b}-\hat{a}^{\dagger} \hat{b} e^{i 2 \theta_{1}}-\hat{b}^{\dagger} \hat{a} e^{-i 2 \theta_{1}}\right] .
\end{aligned}
$$

Detector $A_{\|}$measured the beat intensity of the transmitted component at PBS1 as:

$$
\begin{gathered}
I_{A_{\|}}\left(\theta_{1 \|}\right)=\left\langle\alpha, \beta\left|\hat{c}_{1}^{\prime \prime} \hat{c}_{1}^{\prime \prime}\right| \alpha, \beta\right\rangle \\
=\eta_{A}\left\{|\alpha|^{2}+|\beta|^{2}-|\alpha||\beta| \cos \left(2 \theta_{1}+\phi_{\beta}-\phi_{\alpha}\right)\right\} .
\end{gathered}
$$

The reflected component where $\theta_{1 \perp}=\theta_{1 \|}+90^{\circ}$ at PBS1 and were measured by detector $A_{\perp}$ can be written as

$$
\begin{aligned}
I_{A_{\perp}}\left(\theta_{1 \perp}\right) & =\left\langle\alpha, \beta\left|\hat{c}_{1}^{\prime \prime} \hat{c}_{1}^{\prime \prime}\right| \alpha, \beta\right\rangle \\
& =\eta_{A}\left\{|\alpha|^{2}+|\beta|^{2}+|\alpha||\beta| \cos \left(2\left(\theta_{1}+90^{\circ}\right)+\phi_{\beta}-\phi_{\alpha}\right)\right\} \\
& =\eta_{A}\left\{|\alpha|^{2}+|\beta|^{2}+|\alpha||\beta| \cos \left(2 \theta_{1}+\phi_{\beta}-\phi_{\alpha}\right)\right\}
\end{aligned}
$$

The balanced homodyne detector A measured beat intensity as 


$$
A_{B H D}=I_{A_{\|}}\left(\theta_{1 \|}\right)-I_{A_{\perp}}\left(\theta_{1 \perp}\right)=2 \eta_{A}|\alpha||\beta| \cos \left(2 \theta_{1}+\phi_{\beta}-\phi_{\alpha}\right)
$$

Since the $B_{B H D}$ remained unchanged for the beam 2 as shown in Fig. 3.1, we obtained the second bipartite correlation function by multiply the balanced homodyne beat intensities of detectors $\mathrm{A}$ and $\mathrm{B}$ as,

$$
\begin{aligned}
\left\langle A_{B H D} \cdot B_{B H D}\right\rangle & =4 \eta_{A} \eta_{B}|\alpha|^{2}|\beta|^{2} \cos 2\left(\theta_{1}-\theta_{2}\right) \\
& \propto \cos 2\left(\theta_{1}-\theta_{2}\right)
\end{aligned}
$$

\subsubsection{Correlation function $\phi^{-}=-\cos 2\left(\theta_{1}+\theta_{2}\right)$}

Third bipartite correlation function $\phi^{+}$can be obtained by using the same setup for bipartite correlation function $\Psi^{+}$with now the QWP is rotated at $-45^{\circ}$ as shown in Fig. 3.3.

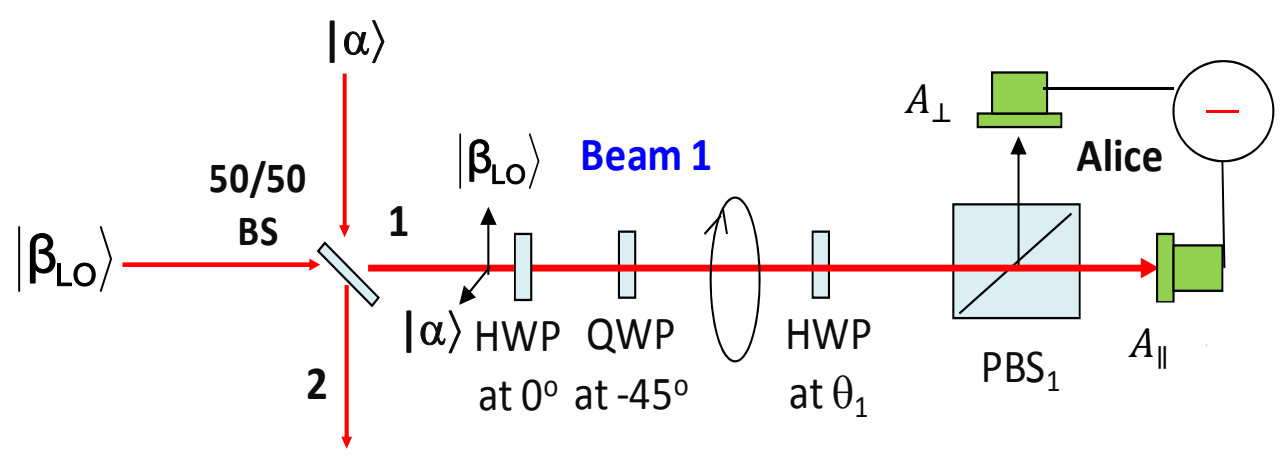

Figure 3.3. Experiment setup used for demonstration of Correlation function $\phi^{+}=$ $\cos 2\left(\theta_{1}-\theta_{2}\right)$

After the QWP the field operators $\hat{c}_{1}$ will be transformed as, 


$$
\begin{aligned}
\hat{c}_{H W P} \rightarrow \hat{c}_{1}^{\prime} & =\frac{1}{\sqrt{2}}\left[\begin{array}{ll}
1 & i \\
i & 1
\end{array}\right] \frac{1}{\sqrt{2}}\left(\hat{a}\left[\begin{array}{c}
-\boldsymbol{i} \\
\mathbf{0}
\end{array}\right]+\hat{b}\left[\begin{array}{c}
\mathbf{0} \\
-\mathbf{1}
\end{array}\right]\right) \\
& =\frac{1}{2}\{i(-\hat{a}-\hat{b}) \mathbf{x}+(\hat{a}-\hat{b}) \mathbf{y}\} .
\end{aligned}
$$

After the polarization analyzer, the field operator for the transmitted component of PBS1 becomes

$$
\begin{array}{r}
\hat{c}_{1}^{\prime} \rightarrow \hat{c}_{1}^{\prime \prime}=\hat{c}_{1}^{\prime} \cdot \hat{e}_{1}=\frac{1}{2}\{i(-\hat{a}-\hat{b}) \mathbf{x}+(\hat{a}-\hat{b}) \mathbf{y}\} \cdot\left(\cos \theta_{1} \mathbf{x}+\sin \theta_{1} \mathbf{y}\right) \\
\hat{c}_{1}^{\prime \prime}=\frac{1}{2}\left[\hat{a}\left(-i \cos \theta_{1}+\sin \theta_{1}\right)+\hat{b}\left(-i \cos \theta_{1}-\sin \theta_{1}\right)\right]
\end{array}
$$

Photon number operator of the transmitted component of at PBS1 is written as

$$
\begin{aligned}
\hat{c}_{1}^{\prime \prime}{ }^{\dagger} \hat{c}_{1}^{\prime \prime}= & \frac{1}{2}\left[\hat{a}\left(-i \cos \theta_{1}+\sin \theta_{1}\right)+\hat{b}\left(-i \cos \theta_{1}-\sin \theta_{1}\right)\right] \\
& \times \frac{1}{2}\left[\hat{a}\left(i \cos \theta_{1}+\sin \theta_{1}\right)+\hat{b}\left(i \cos \theta_{1}-\sin \theta_{1}\right)\right] \\
= & \frac{1}{4}\left[\hat{a}^{\dagger} \hat{a}+\hat{b}^{\dagger} \hat{b}+\hat{a}^{\dagger} \hat{b} e^{-i 2 \theta_{1}}+\hat{b}^{\dagger} \hat{a} e^{i 2 \theta_{1}}\right]
\end{aligned}
$$

Detectors $A_{\|}$and $A_{\perp}$ measured the beat intensities of the transmitted and reflected components at PBS1

$$
\begin{aligned}
I_{A_{\|}}\left(\theta_{1 \|}\right) & =\left\langle\alpha, \beta\left|\hat{c}_{1}^{\prime \prime} \hat{c}_{1}^{\prime \prime}\right| \alpha, \beta\right\rangle \\
& =\eta_{A}\left\{|\alpha|^{2}+|\beta|^{2}+|\alpha||\beta| \cos \left(2 \theta_{1}-\phi_{\beta}+\phi_{\alpha}\right)\right\}
\end{aligned}
$$

and 


$$
\begin{aligned}
I_{A_{\perp}}\left(\theta_{1 \perp}\right) & =\left\langle\alpha, \beta\left|\hat{c}_{1}^{\prime \prime \dagger} \hat{c}_{1}^{\prime \prime}\right| \alpha, \beta\right\rangle \\
& =\eta_{A}\left\{|\alpha|^{2}+|\beta|^{2}-|\alpha||\beta| \cos \left(2 \theta_{1}-\phi_{\beta}+\phi_{\alpha}\right)\right\} .
\end{aligned}
$$

The balanced homodyne detector A measured beat intensity

$$
A_{B H D}=I_{A_{\|}}\left(\theta_{1 \|}\right)-I_{A_{\perp}}\left(\theta_{1 \perp}\right)=2 \eta_{A}|\alpha||\beta| \cos \left(2 \theta_{1}-\phi_{\beta}+\phi_{\alpha}\right) .
$$

Now, multiplying the balanced homodyne beat intensities of detectors A and B as,

$$
\begin{aligned}
\left\langle A_{B H D} \cdot B_{B H D}\right\rangle & =4 \eta_{A} \eta_{B}|\alpha|^{2}|\beta|^{2} \cos 2\left(\theta_{1}+\theta_{2}\right) \\
\propto & -\cos 2\left(\theta_{1}+\theta_{2}\right) .
\end{aligned}
$$

Which is third bipartite correlation function $\phi^{-}=-\cos 2\left(\theta_{1}+\theta_{2}\right)$.

\subsubsection{Correlation function $\phi^{+}=\cos 2\left(\theta_{1}+\theta_{2}\right)$}

The last bipartite correlation function $\phi^{+}$can be obtained by simply removing the HWP at $0^{\circ}$ in front of the QWP is depicted in the Fig.3.4.

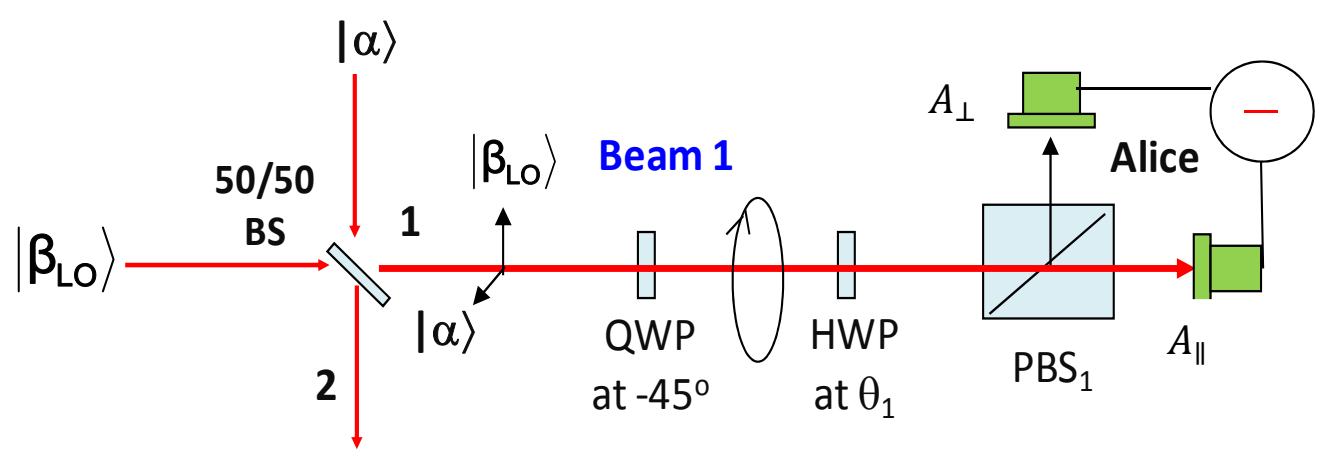

Figure 3.4. Experiment setup used for the demonstration of Correlation function $\phi^{-}=\cos 2\left(\theta_{1}-\theta_{2}\right)$ 
After the QWP the field operators $\hat{c}_{1}$ will be transformed as,

$$
\begin{aligned}
\hat{c}_{1} \rightarrow \hat{c}_{1}^{\prime}= & \frac{1}{\sqrt{2}}\left[\begin{array}{ll}
1 & i \\
i & 1
\end{array}\right] \frac{1}{\sqrt{2}}\left(\hat{a}\left[\begin{array}{l}
\mathbf{1} \\
\mathbf{0}
\end{array}\right]+i \hat{b}\left[\begin{array}{l}
\mathbf{0} \\
\mathbf{1}
\end{array}\right]\right) \\
& =\frac{1}{2}\{(\hat{a}-\hat{b}) \mathbf{x}+i(\hat{a}+\hat{b}) \mathbf{y}\} .
\end{aligned}
$$

After the polarization analyzer, the field operator for the transmitted component of PBS1 is transformed into

$$
\begin{aligned}
\hat{c}_{1}^{\prime} \rightarrow \hat{c}_{1}^{\prime \prime}=\hat{c}_{1}^{\prime} \cdot \hat{e}_{1}= & \frac{1}{2}\{(\hat{a}-\hat{b}) \mathbf{x}+i(\hat{a}+\hat{b}) \mathbf{y}\} \cdot\left(\cos \theta_{1} \mathbf{x}+\sin \theta_{1} \mathbf{y}\right) \\
\hat{c}_{1}^{\prime \prime} & =\frac{1}{2}\left[\hat{a}\left(\cos \theta_{1}+i \sin \theta_{1}\right)+\hat{b}\left(-\cos \theta_{1}+i \sin \theta_{1}\right)\right] .
\end{aligned}
$$

The photon number operator of the transmitted component at PBS1 becomes

$$
\begin{aligned}
\hat{c}_{1}^{\prime \prime \dagger} \hat{c}_{1}^{\prime \prime}= & \frac{1}{2}\left[\hat{a}\left(\cos \theta_{1}+i \sin \theta_{1}\right)+\hat{b}\left(-\cos \theta_{1}+i \sin \theta_{1}\right)\right] \\
& \times \frac{1}{2}\left[\hat{a}\left(\cos \theta_{1}-i \sin \theta_{1}\right)+\hat{b}\left(-\cos \theta_{1}-i \sin \theta_{1}\right)\right] \\
= & \frac{1}{4}\left[\hat{a}^{\dagger} \hat{a}+\hat{b}^{\dagger} \hat{b}-\hat{a}^{\dagger} \hat{b} e^{-i 2 \theta_{1}}-\hat{b}^{\dagger} \hat{a} e^{i 2 \theta_{1}}\right]
\end{aligned}
$$

Detectors $A_{\|}$and $A_{\perp}$ measure the beat intensities of transmitted and reflected components at PBS1 as

$$
I_{A_{\|}}\left(\theta_{1 \|}\right)=\left\langle\alpha, \beta\left|\hat{c}_{1}^{\prime \prime \dagger} \hat{c}_{1}^{\prime \prime}\right| \alpha, \beta\right\rangle
$$




$$
=\eta_{A}\left\{|\alpha|^{2}+|\beta|^{2}-|\alpha||\beta| \cos \left(2 \theta_{1}-\phi_{\beta}+\phi_{\alpha}\right)\right\},
$$

and

$$
\begin{aligned}
I_{A_{\perp}}\left(\theta_{1 \perp}\right) & =\left\langle\alpha, \beta\left|\hat{c}_{1}^{\prime \dagger} \hat{c}_{1}^{\prime \prime}\right| \alpha, \beta\right\rangle \\
& =\eta_{A}\left\{|\alpha|^{2}+|\beta|^{2}+|\alpha||\beta| \cos \left(2 \theta_{1}-\phi_{\beta}+\phi_{\alpha}\right)\right\} .
\end{aligned}
$$

Then, the balanced homodyne detectors A measured beat intensity as

$$
A_{B H D}=I_{A_{\|}}\left(\theta_{1 \|}\right)-I_{A_{\perp}}\left(\theta_{1 \perp}\right)=-2 \eta_{A}|\alpha||\beta| \cos \left(2 \theta_{1}-\phi_{\beta}+\phi_{\alpha}\right)
$$

Now, multiplying the balanced homodyne beat intensities of detectors A and B we obtained fourth bipartite correlation function $\phi^{-}$as:

$$
\begin{aligned}
A_{B H D} \cdot B_{B H D}= & -2 \eta_{A}|\alpha||\beta| \cos \left(2 \theta_{1}-\phi_{\beta}+\phi_{\alpha}\right) \cdot-2 \eta_{B}|\alpha||\beta| \cos \left(2 \theta_{2}+\phi_{\beta}-\phi_{\alpha}\right) \\
\left\langle A_{B H D} \cdot B_{B H D}\right\rangle & =4 \eta_{A} \eta_{B}|\alpha|^{2}|\beta|^{2} \cos 2\left(\theta_{1}+\theta_{2}\right) \\
& \propto \cos 2\left(\theta_{1}+\theta_{2}\right)
\end{aligned}
$$

\subsection{Detection apparatus}

\subsubsection{Photodetectors and Transimpedance amplifier}

The optical weak coherent states is detected and converted into photocurrent by using two p-i-n photodiodes with active area diameter of $0.5 \mathrm{~mm}$. Both photodiodes (D1 and D2) are connected in balanced detection setup. These p-i-n photodiodes were 
chosen due to their advantages in low intrinsic dark current (12 nA), small intrinsic capacitance $(35 \mathrm{pF})$, high linearity at low optical power, and excellent responsivity $(0.95 \mathrm{~A} / \mathrm{W})$ at wavelength, near $1.55 \mu \mathrm{m}$ region. The photodiodes are each reversebiased by $3 \mathrm{~V}$ batteries in order to improve their response time. The photodiodes are fitted through an aluminum casing and mounted on the circuit board together with other electronic components. Then, the subtracted photocurrent was be fed to a transimpedance amplifier and converted into voltage as shown in Fig. 3.5.

We employed a low noise operational amplifier to build the transimpedance amplifier circuit, which was used to convert the photocurrent into voltage. Fig. 3.5 shows our circuit design using a $2 \mathrm{pF}$ feedback capacitance and a $15 \mathrm{k} \Omega$ transimpedance gain which is estimated to produce the $3 \mathrm{db}$ gain and a bandwidth of about $20 \mathrm{Mhz}$. The $2 \mathrm{pF}$ capacitance also helped to eliminate the resonance noise and stabilize the circuit by balancing the photodiode's intrinsic capacitance. The output DC voltage fluctuation of the circuit is minimized by connecting the $50 \Omega$ resistor between ground and non- inverting input.

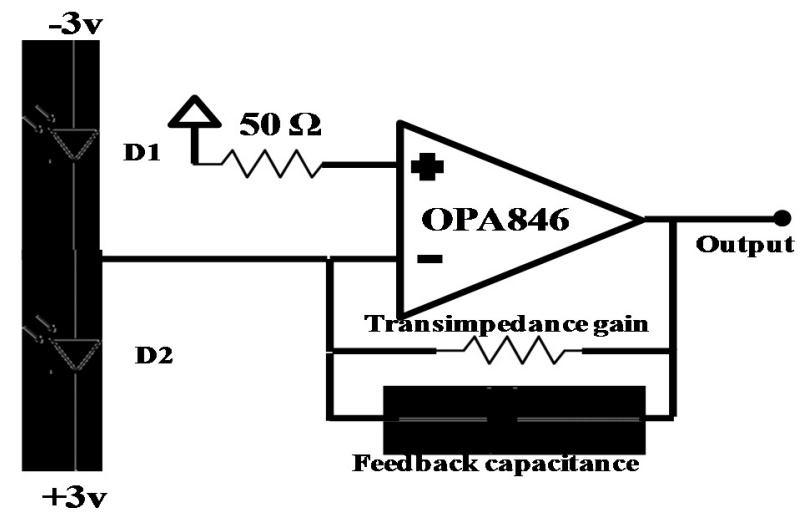

Figure 3.5. Schematic diagram of balanced detection and transimpedance amplifier circuit 


\subsubsection{Oscilloscope and Spectrum analyzer}

The transimpedance amplifier output is then fed to a digital oscilloscope $(500 \mathrm{MHz}$ bandwidth) for the data acquisition. The data acquisition systems on the outputs from both observers in the experiment were synchronized and triggered by the same source. This was crucial to measurement the correlation of both observers. The electronic noise level and shot noise of the optical field in frequency spectrum was measured by spectrum analyzer ( $2 \mathrm{GHz}$ bandwidth). From electronic noise level and shot noise measurements, we can determine the minimum optical power for weak coherent states' and weak LO field. This will ensure information of weak coherent states' correlations was concealed by the electronic noise and quantum phase noise.

\subsubsection{Lock in amplifier}

An analog lock-in amplifier (dual phase) was used for the implementation of the bit correlation between two observers. The transimpedance amplifier output was fed directly into lock-in amplifier. The lock-in amplifier was referenced from the driver of the piezoelectric transducer that modulated the local oscillator field. The same reference signal will be used for all bit correlation measurements. 


\subsection{Balanced Homodyne detection}

Balanced homodyne detector was utilized in the detection scheme for the weak coherent light fields for optical communication. The term homodyne means that local oscillator is derived from the same source as the signal before the modulating process.

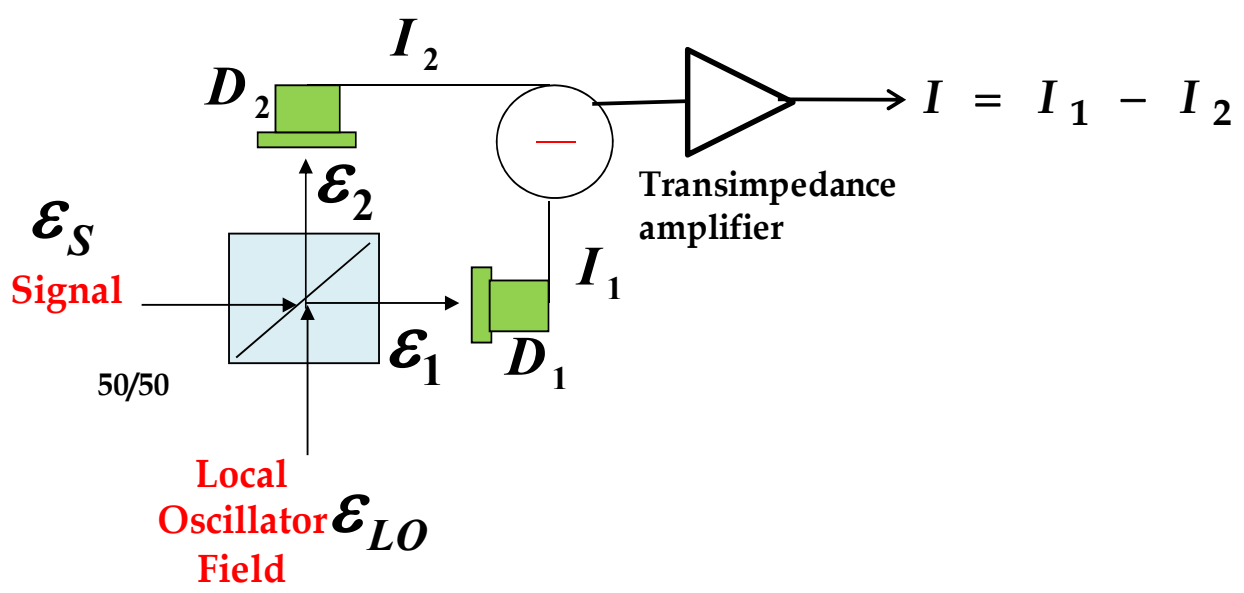

Figure 3.6. Balanced Homodyne detection.

Balanced homodyne detector consists of a 50/50 beam splitter, two photo detectors, a local oscillator field and a transimpendance amplifier. It has two input ports. The signal field was sent into one of the input port, while local oscillator field was sent through another port. The signal and LO fields were optically mixed at the beam splitter. Normally, the local oscillator field can be derived from the same laser source as signal field, having the same frequency and a well-defined phase with respect to the signal field. Mixing the local oscillator field and signal field allowed to enhance the signal field detection. Superimposed local oscillator field and weak coherent states were detected by photodiodes $\mathrm{D}_{1}$ and $\mathrm{D}_{2}$. The photodiodes are 
connected together in such a way that the output is equals to the difference of the photocurrents generated by photodiodes $D_{1}$ and $D_{2}$. The emerging output fields $\varepsilon_{1}$ and $\varepsilon_{2}$ are the superposition of signal and local oscillator field:

$$
\begin{gathered}
\varepsilon_{1}=\frac{1}{\sqrt{2}}\left(\varepsilon_{L O}+\varepsilon_{\text {signal }}\right) \\
\varepsilon_{2}=\frac{1}{\sqrt{2}}\left(\varepsilon_{L O}-\varepsilon_{\text {signal }}\right)
\end{gathered}
$$

where $\varepsilon_{L O}$ and $\varepsilon_{\text {signal }}$ are the local oscillator and signal field respectively. Photocurrents produced by the output fields $\varepsilon_{1}$ and $\varepsilon_{2}$ were given as

$$
\begin{gathered}
I_{1}=\left|\varepsilon_{1}\right|^{2}=\varepsilon_{1} \varepsilon_{1}{ }^{*} \\
I_{2}=\left|\varepsilon_{2}\right|^{2}=\varepsilon_{2} \varepsilon_{2}{ }^{*} .
\end{gathered}
$$

Hence, the output of the balanced homodyne detector is given as,

$$
I_{2}-I_{1}=2 \varepsilon_{S} \varepsilon_{L O}
$$

The signal and local oscillator fields were derived from the same laser source with relative phase $\phi$. By considering only the real part of the signal and local oscillator fields, it can be written as,

$$
\begin{aligned}
\varepsilon_{S} & =A_{\varepsilon_{S}} \cos (\omega t) \\
\varepsilon_{L O} & =A_{\varepsilon_{L O}} \cos (\omega t+\phi)
\end{aligned}
$$


Where $A_{\varepsilon_{S}}$ and $A_{\varepsilon_{L O}}$ are the amplitude for signal and local oscillator fields, $\omega$ is optical frequency, $\phi$ is relative phase between the fields. Hence the output of the balanced homodyne detector is given by

$$
I_{1}-I_{2}=\left|A_{\varepsilon_{S}}\right|\left|A_{\varepsilon_{L O}}\right|\{\cos (\phi)+\cos (2 \omega t+\phi)\} .
$$

The second term in the Eq.(47) is the fast varying term beyond the detection bandwidth of the oscilloscope. Therefore, the output of the balanced homodyne detector is phase dependence, which is given by,

$$
I_{1}-I_{2} \propto\left|A_{\varepsilon_{S}}\right|\left|A_{\varepsilon_{L O}}\right| \cos (\phi) .
$$

One of the main advantages of using balanced homodyne detector is the higher signal to noise ratio compared to a single detector. For example, intensity fluctuations of the laser would affect the measurement by a single detector. Contrary, any changes in intensity will be canceled by the subtraction of the photocurrent with an ideal balanced homodyne detector.

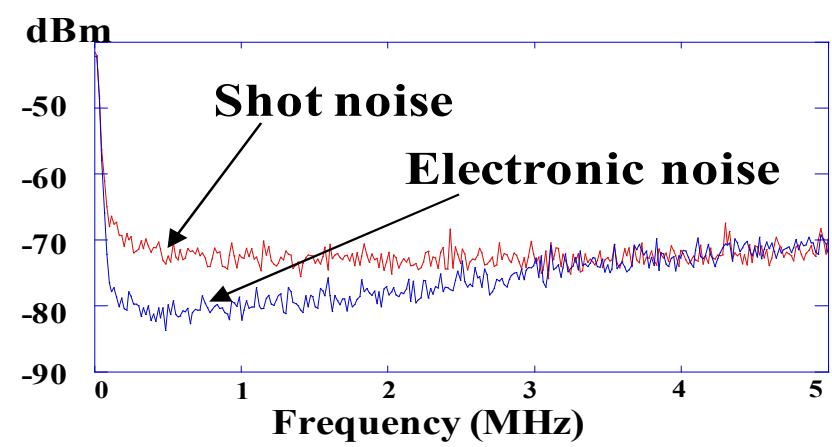

Figure 3.7. Frequency spectrum of balanced homodyne detector indicating the electronics noise level without any light and the shot noise level with the presence of the local oscillator field. 
Due to the Poissonian statistics of the coherent light, polarization dependence of the beam splitter and random splitting process in the 50/50 beam splitter; fluctuations in intensity cannot be completely removed. Therefore, even with the presence of only local oscillator field, the balanced homodyne detector will have a shot noise level above the electronics noise level as depict in Fig.3.7, limiting the signal to noise ratio.

\subsection{Results}

\subsubsection{Weak Coherent States Interference}

To verify the above analysis and measurement method for weak coherent states, we first perform the experiment by using a strong LO field and a weak coherent state with average power of $1 \mathrm{~mW}$ and $0.03 \mathrm{~mW}$, respectively. Fig.3.8 (a) shows the spectrum of the shot noise of the strong LO field and weak coherent state, and the electronic noise of our detection system. In this experiment, the relative angle between Alice's and Bob's analyzers was equals to zero. The beat signal intensities at detectors A and B are shown in Fig.3.8 (b) and Fig.3.8 (c). The discontinuities of the beat signals are due to the ramping of the PZT.

With these large mean photon fluxes, the interference signals are stable as predicted by the coherent states with large mean photon number. The product of the beat intensities is shown in Fig.3.8 (d), which is negative in averaged. This indicates single-field interferences at detectors A and B are anticorrelated. In the experiment, the beat intensities at detectors A and B are recorded in computers were recorded by 
computer connected to the digital oscilloscope. The data acquisition of detectors A and B was synchronized and triggered at the same time in order to obtain the meaningful correlation from the multiplied signals. Next, the laser was attenuated to obtain weak LO field and weak coherent state with average power of $0.001 \mathrm{~mW}$ each. Fig. 3.9 (a) depicts the spectrum of the shot noise levels of the weak LO field and coherent state, and the electronic noise of our detection system. All the average optical powers reported in this work are measured just before the PBS1 (PBS2). Fig. 3.9 (a) shows that the shot noise of weak LO field. It falls almost on the same level as electronic noise spectrum. We observed that the beat intensities at detectors A and B as shown in Fig. 3.9. (b) and Fig. 3.9. (c) with the interference signals hidden or protected by the noises of the signal. These include shot noise of the LO field, quantum phase noise $\phi_{\alpha}$ of the weak coherent state, and electronic noises as predicted by Eqs. 3.16 and 3.17. Quantum phase noise $\phi_{\alpha}$ is due photon number fluctuation of weak coherent state.

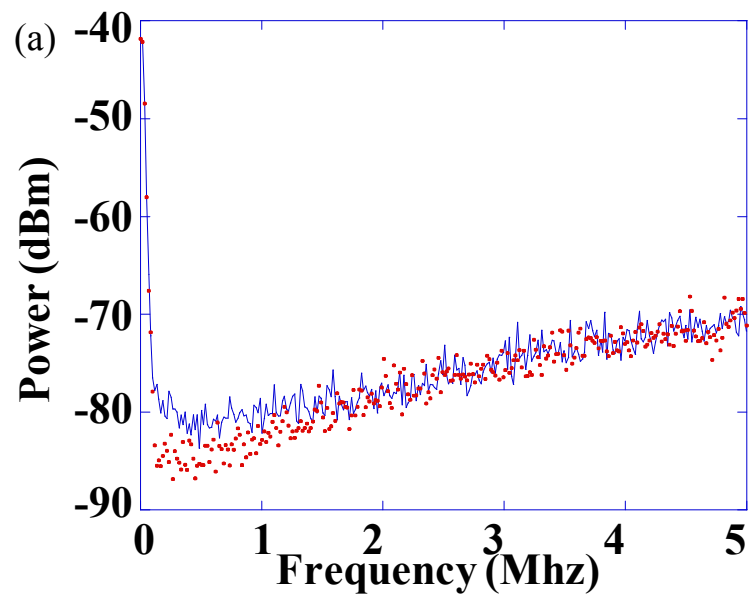

(b)
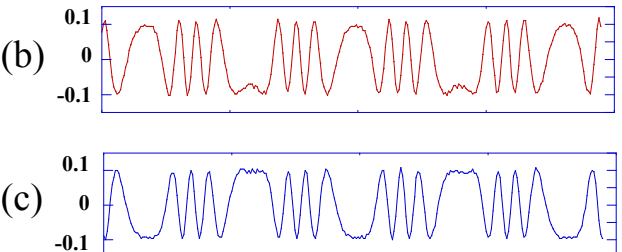

(d)

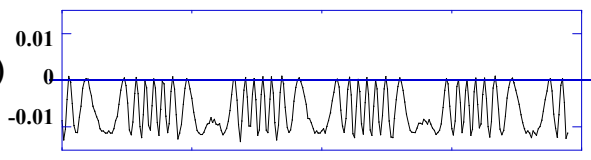

Time/ms

Figure 3.8. (a) Shot noise (blue solid line) of the weak LO field (1 $\mathrm{mW})$ plus coherent state $(0.03 \mathrm{~mW})$ and electronic noise level (red dots); the corresponding 
beat signals for $\theta_{1}=\theta_{2}$ at detector A (b) and detector B (c), and their multiplied signal (d)

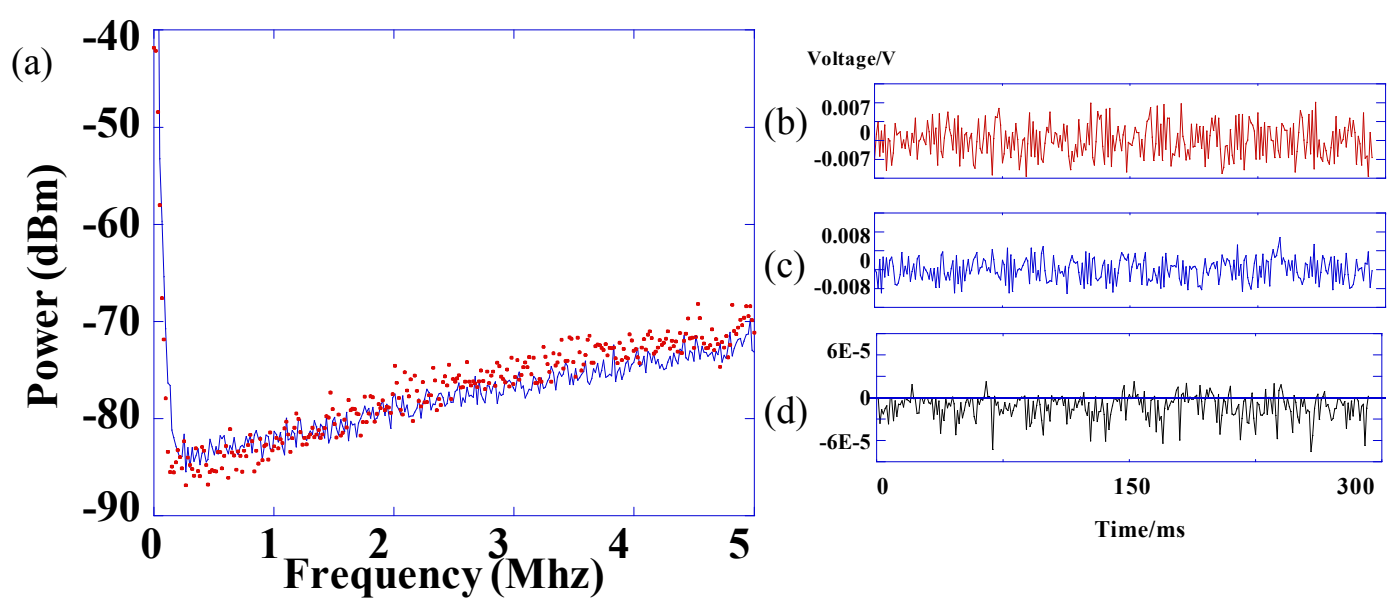

Figure 3.9 (a) Shot noises (blue solid line) of the weak LO field $(0.001 \mathrm{~mW})$ plus weak coherent state $(0.001 \mathrm{~mW})$ and electronic noise level (red dots); the corresponding beat signals for $\theta_{1}=\theta_{2}$ at detector A (b) and detector B (c), and their multiplied signal (d)

The raw data are multiplied together and shown in Fig. 3.9(d). Fig. 3.9(d) shows that the multiplied signal is are anticorrelated. This is predicted by correlation function $-\cos 2\left(\theta_{1}-\theta_{2}\right)=-1$ when the relative angle is set to $\theta_{1}-\theta_{2}=0$.

The multiplied beat intensity consists of two parts: coherent and noise interferences. The coherent interference part contains the term $-4 \eta_{A} \eta_{B}\left\{|\alpha|^{2}|\beta|^{2} \cos 2\left(\theta_{1}-\theta_{2}\right)\right\}$. Noise interference part contains the term $4 \eta_{A} \eta_{B}\left\{\cos 2\left(\theta_{2}+\theta_{1}+\phi_{\beta}-\phi_{\alpha}\right)\right\}$ which is averaged to zero because of the periodic of the LO phase, $\phi_{\beta}=\{0 \rightarrow 2 \pi\}$. The multiplied beat intensity is protected by the quantum phase noise $\phi_{\alpha}[\underline{108]}$. Electronic noise in our measurement created statistical errors in the mean-value measurement of the coherent part of the multiplied signal. 
(a)

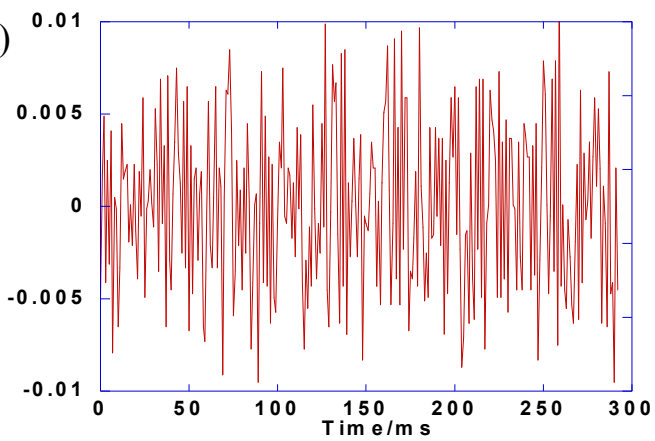

(b)
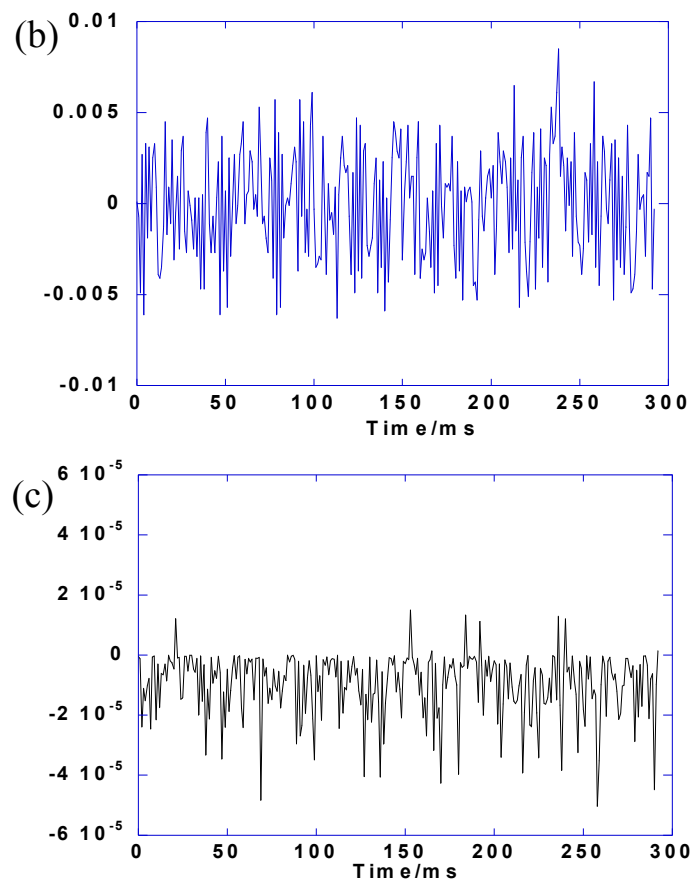

(d)

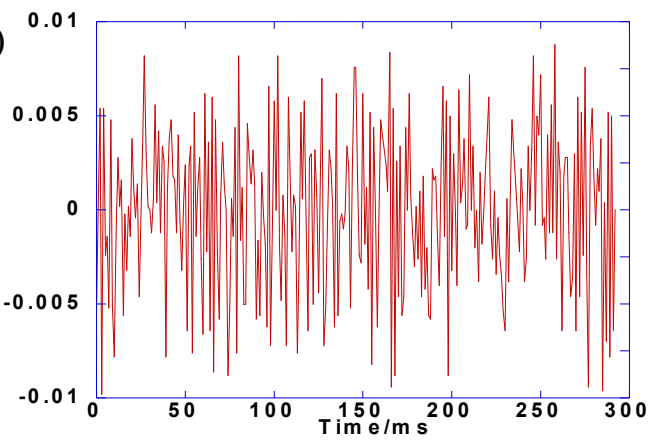

(e)

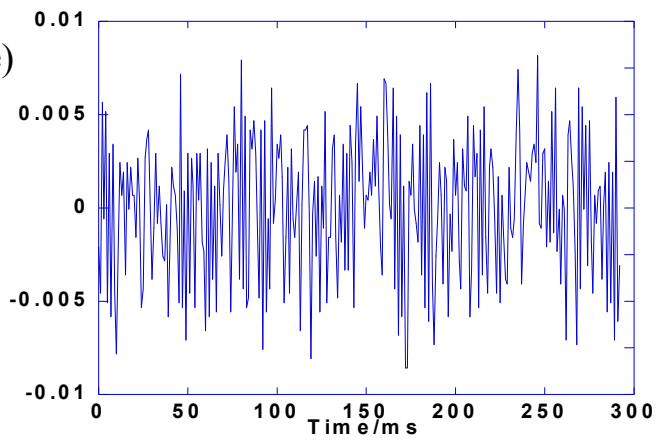

(f)

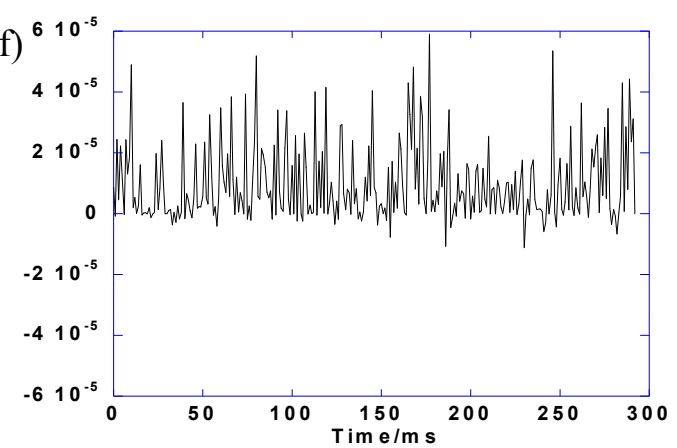

Figure 3.10. The experimental observation of the correlation functions i) $\Psi^{-}=$ $-\cos 2\left(\theta_{1}-\theta_{2}\right)$ for the case $\theta_{1}=\theta_{2}$. The observed random noise beat signal in (a) detector A, (b) detector B. (c) Multiplied beat signals. ii) $\Psi^{-}=\cos 2\left(\theta_{1}-\theta_{2}\right)$ for the case $\theta_{1}=\theta_{2}$. The observed random noise beat signal in (d) detector $\mathrm{A}$, (e) detector $\mathrm{B}$, (f) Multiplied beat signals

As an illustration of our experimental observation for the correlation function $\Psi^{-}=-\cos 2\left(\theta_{1}-\theta_{2}\right)$, we take a single measurement of the anti-correlated beat intensities at detectors $\mathrm{A}$ and $\mathrm{B}$ for $\theta_{1}=\theta_{2}$ as shown in Fig. 3.10.(a) and Fig. 3.10.(b) respectively. The mean value of beat intensities at detectors A and B are 
zero as expected. The multiplied anti-correlated signal is shown in Fig. 3.10.(c) and has the maximum obtainable mean value in the experiment, which is a negative value. The positive spikes, observed in the multiplied signal are possibly induced by the additional noise from the ambient environment or detection apparatus, which will affect the bipartite correlation. For the case of correlation function $\Psi^{+}=$ $\cos 2\left(\theta_{1}-\theta_{2}\right)$ and $\theta_{1}=\theta_{2}$, the beat intensities in detectors A and B are shown in Fig. 3.10. (d) and Fig. 3.10. (e) respectively. For this case, the multiplied signal is shown in Fig. 3.10. (f), where its mean value is positive.

\subsubsection{Weak Coherent states Bipartite correlations (CSBC)}

By using the experiment setup as decribed in section 3.3, we are able to generate 4 types of bipartite correlation, given as

$$
\begin{aligned}
& \Psi^{-}=-\cos 2\left(\theta_{1}-\theta_{2}\right) \\
& \Psi^{+}=\cos 2\left(\theta_{1}-\theta_{2}\right) \\
& \phi^{-}=-\cos 2\left(\theta_{1}+\theta_{2}\right) \\
& \phi^{+}=\cos 2\left(\theta_{1}+\theta_{2}\right)
\end{aligned}
$$

To verify the analysis discussed in section 2 , we performed systematic analysis of the proposed experiment. We used a piezoelectric transducer (PZT) to modulate the phase of a weak light field. Then, all 4 types of correlation function were obtained by manipulation of experiment setup as discussed in previous section. We normalized each correlation function $\Psi^{-}=-\cos 2\left(\theta_{1}-\theta_{2}\right)$ to its maximum obtainable value, when $\theta_{1}=\theta_{2}$. Fig.3.11 shows the normalized correlation function 
$\pm \cos 2\left(\theta_{1} \pm \theta_{2}\right)$ as a function of the relative projection angle of the analyzer $\mathrm{A}$ and B. The blue line is the predicted theoretical value while the red circle with the error bar is the experimental data. For each data point, we took ten data points of the multiplied signal and obtain the average mean value. Each measurement was obtained by setting the polarization angle of the analyzer A unchanged and varies the polarization angle of analyzer B. The error bar is mainly due to the electronic noise and temperature dependence of polarization optics. The deviation of experimental data from theoretical prediction is mainly due to the resolution of the rotation angle of the polarization analyzer and imperfection of polarization optics.
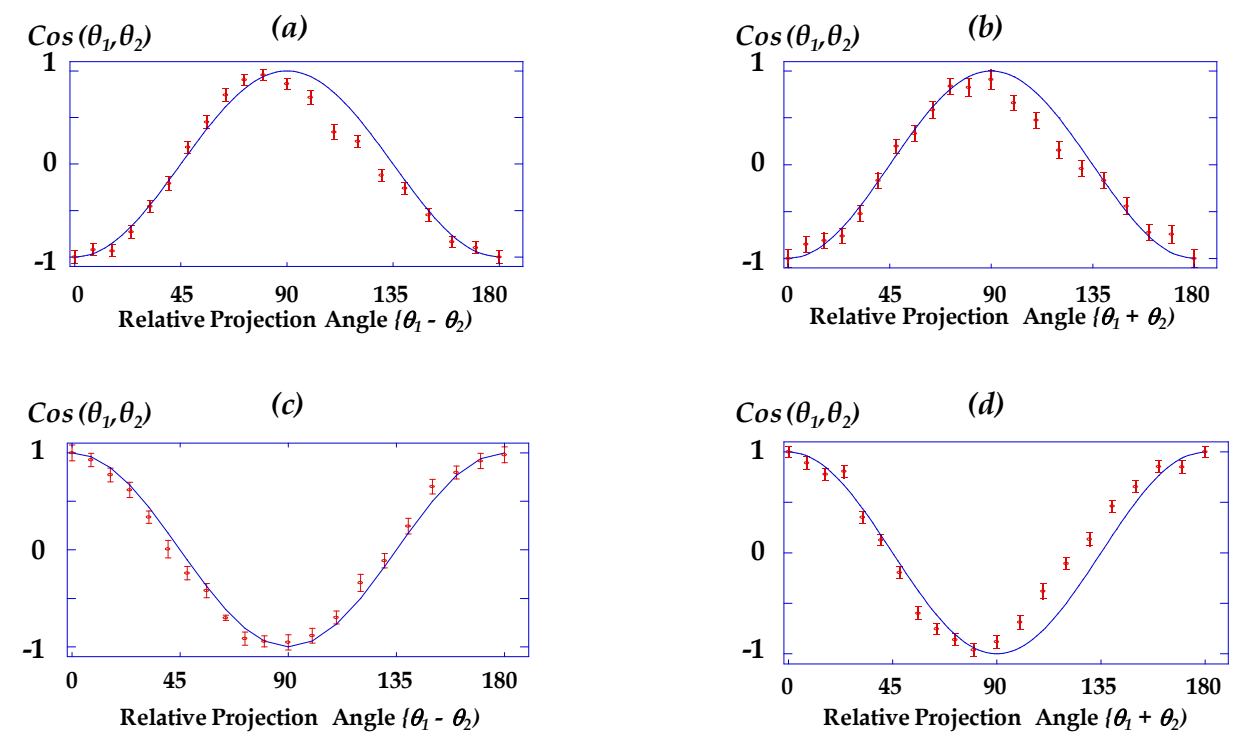

Figure 3.11. Experimental measurement of Bi-partite correlation functions (a) $-\cos 2\left(\theta_{1}-\theta_{2}\right),(\mathrm{b})-\cos 2\left(\theta_{1}+\theta_{2}\right),(\mathrm{c}) \cos 2\left(\theta_{1}-\theta_{2}\right),(\mathrm{d}) \cos 2\left(\theta_{1}+\theta_{2}\right)$ 


\subsubsection{Bit correlations measurement}

After we establish or choose one of the coherent state bipartite correlation functions between observer A and B, we measured bits correlations between them. To perform this measurement for the established correlation function of $\Psi^{-}=-\cos 2\left(\theta_{1}-\theta_{2}\right)$, we modulated the piezoelectric transducer to modulate the phase of the local oscillator field at $0.03 \mathrm{~mW}$ to obtain one period of interference signal as shown in Fig.3.12 (a).
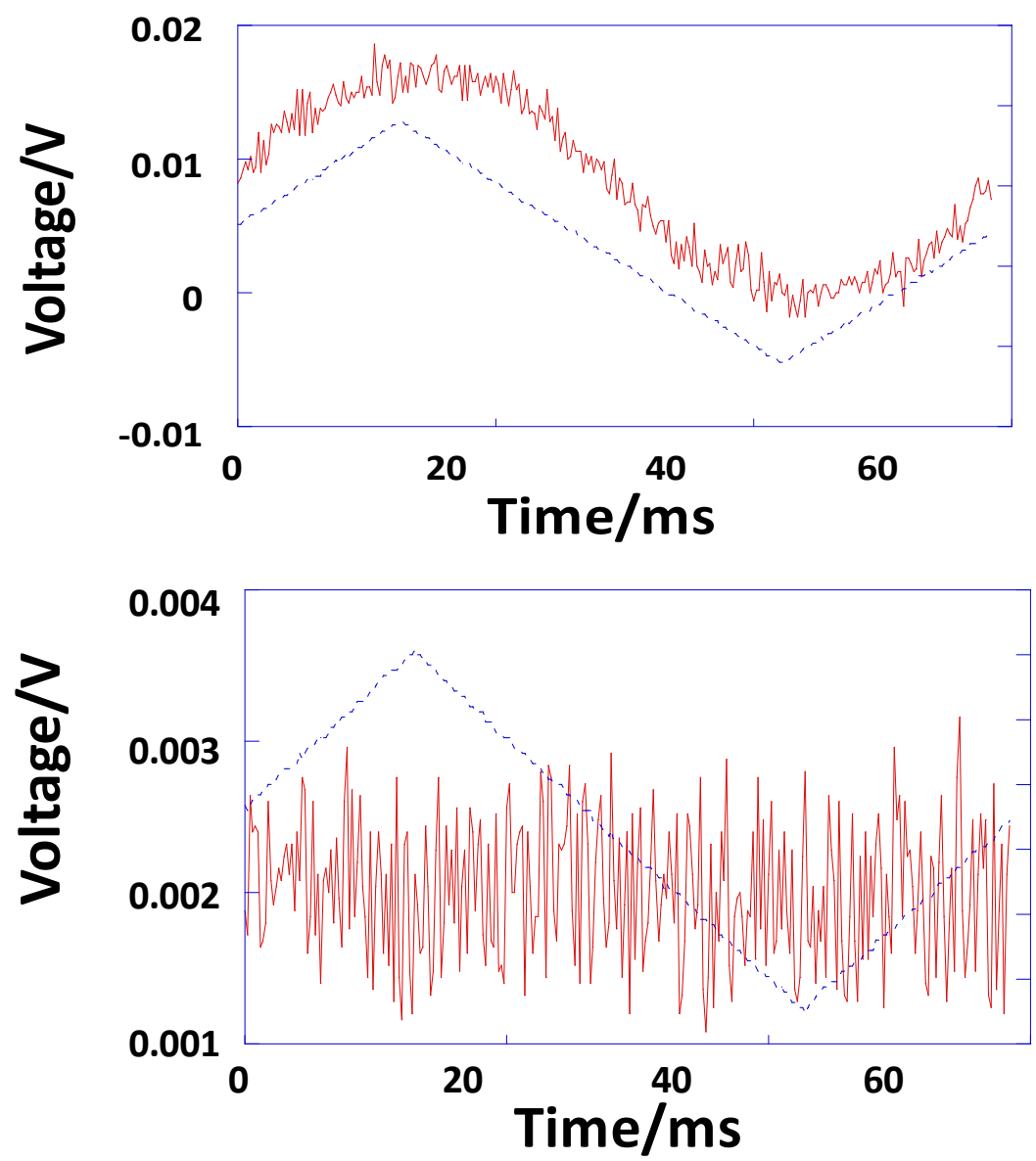

Figure 3.12. a) Single period of interference signal measured at observer A (red solid line) compared to b) piezoelectric driving voltage (blue dashed line), which is used as reference phase in the lock-in amplifier. 
From this interference signal we can determine the reference signal for the lock-in amplifier to measure bits correlations between observer A and B. Then, we reduced the average power of the weak LO field and weak coherent state to 0.00013 $\mathrm{mW}$. As shown in Fig 3.12 (b), the interference signal and phase information is protected by random noise and was explained in previous section.

For the bits correlation measurement, the output of the balanced homodyne beat intensity at detector A is directly connected to a lock-in amplifier. Fig. 8 depicts the experimental setup for bit measurement for observer A and B. To perform this measurement for the established correlation function of $-\cos 2\left(\theta_{1}-\theta_{2}\right)$, we measured quadrature phases of weak coherent state with the step size of $n \pi / 2(n=$ integer). The results are shown in Fig. 4(a) (blue line). Using the same lock-in reference phase in the lock-in amplifier, we measure the quadrature phases of weak coherent state at detector B as shown in Fig. 3.14(a) (dashed red line). In analogous to correlation function of $-\cos 2\left(\theta_{1}-\theta_{2}\right)$, detector $\mathrm{A}$ and $\mathrm{B}$ is anticorrelated as they have opposite bit correlation at the same LO phase as depicted in Fig. 4(a). The positive (negative) quadrature signal is encoded as keys/bits ' 1 ' (' 0 ').

By using the same lock-in reference signal, we established four types of bipartite correlation functions and performed bits correlations for each shared correlation function between two parties. The other three types of correlation functions $-\cos 2\left(\theta_{1}+\theta_{2}\right), \cos 2\left(\theta_{1}+\theta_{2}\right)$, and $\cos 2\left(\theta_{1}-\theta_{2}\right)$ as shown in Figs. 3.14(b), (c), and (d), respectively. 


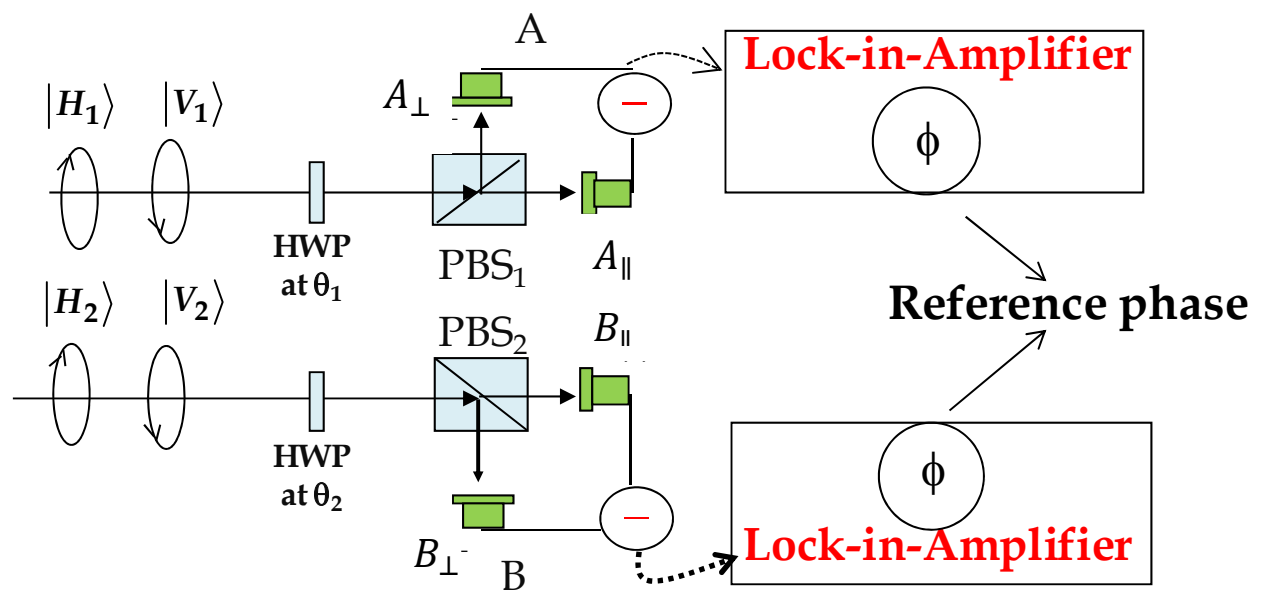

Figure 3.13. Experiment setup for demonstration of the bit generation and measurement
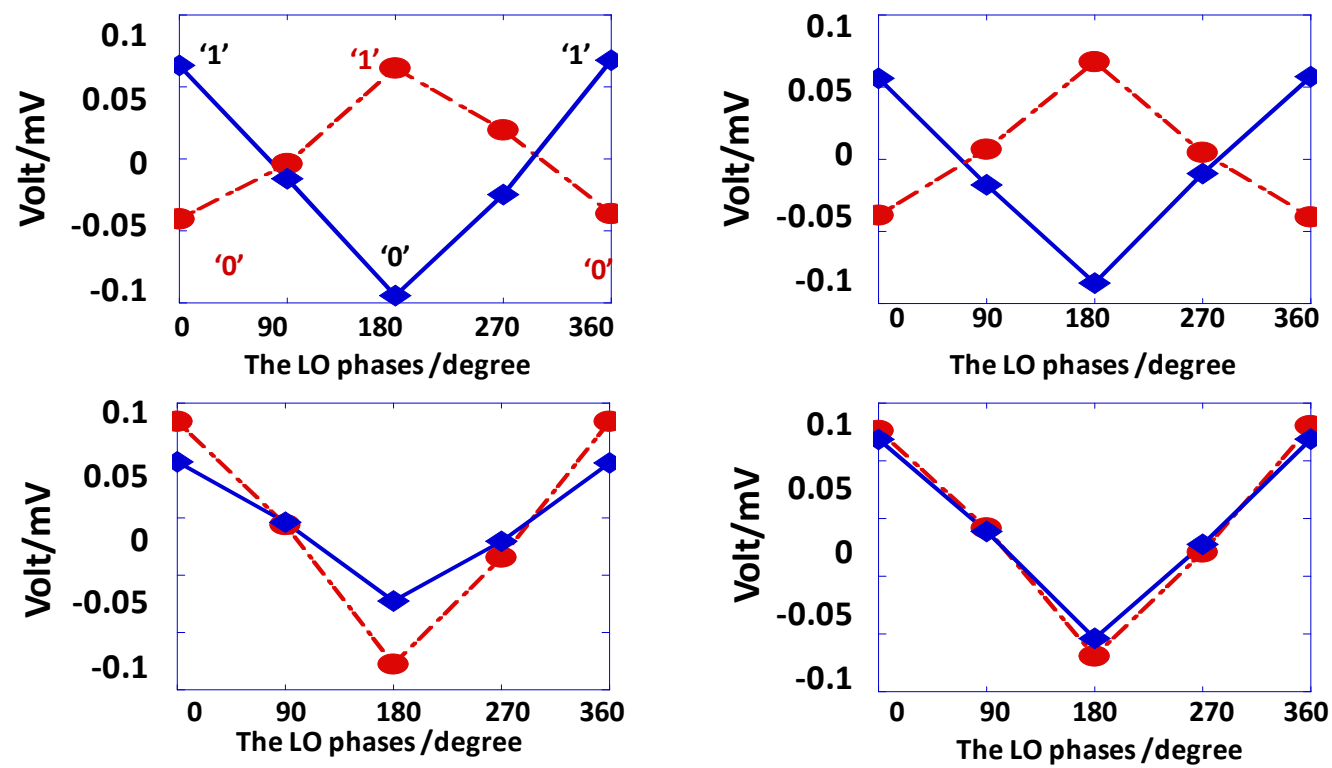

Figure 3.14. Bit correlation of two weak light fields a) $-\cos 2\left(\theta_{1}-\theta_{2}\right)$, (b) $-\cos 2\left(\theta_{1}+\theta_{2}\right)$, (c) $\cos 2\left(\theta_{1}-\theta_{2}\right)$, (d) $\cos 2\left(\theta_{1}+\theta_{2}\right)$ 


\subsection{Discussions}

In this work, we employ a weak local oscillator field in a coherent state to extract intrinsic correlations of weak coherent states between two parties using a balanced homodyne measurement. Briefly, we first prepare a weak coherent state using a highly attenuated laser at telecom wavelength. The weak coherent state is split by a 50-50 beam splitter and sent to Alice and Bob, each has a balanced homodyne detection scheme for measuring his or her coherent state with a weak local oscillator field.

We employ nonlinearity of the post measurement method by multiply two single-field interferences from an individual balanced homodyne measurement. Then, the mean value of the multiplied signal provides raw the correlations of weak coherent states. We normalize the raw data with the mean photon numbers of a weak coherent state and LO field to obtain the coherent state bipartite correlation function. As explained in experiment setup, four types of correlation functions $\pm \cos 2\left(\theta_{1} \pm\right.$ $\theta_{2}$ ) can be prepared by using linear optics devices in Alice or Bob alone, where $\theta_{1}$ and $\theta_{2}$ are the projection angles of the analyzers at Alice and Bob. In the context of quantum communication, this means that Alice can keep her copy of the coherent state and send another copy to Bob. By locally changing the relative phases between her coherent state and weak local oscillator field, her acts will change the correlation functions shared with Bob. Apparently, stability and accurate control of phase and polarization turned out to be the main challenge for the practical implementation 
quantum communication by weak coherent states. The polarization state of the light is not preserved in the typical transmission fiber. Dynamic control of the state of polarization of the light is critical to ensure the reliability the proposed optical communication scheme. Each dynamic polarization controller is bulky and expensive, severely limits the practicality of our scheme [110].

Once we establish one of the four correlation functions between Alice and Bob over a distance of $10 \mathrm{~km}$ through a transmission fiber, we change the phases of the weak local oscillator field $\{0, \pi\}$ for implementing bits correlations between them. For reliable measurement of the encoded signal, both phase and polarization of the weak light field must be stable. Phase locking is another challenging obstacle as well. Phase locking is required between the two orthogonal weak light fields that used to implement the bit correlation between two observers. Without the phase locking, quadrature phases measurement performed by lock-in amplifier is meaningless. Therefore, optical phase-locked loops must be employed for the phase locking of two weak light fields. However, for high data rate optical communication, the delays allowed in the phased-locked loop are so small that phase locking becomes an enormous challenge $[\underline{111}, \underline{112}]$

As a supplement to the DPS-QKD, the phase of the weak LO field can be randomly modulated as $\{0, \pi\}$ at certain frequency. Then the bit-key correlations can be realized based on the established CSBC shared by both parties. Since the established CSBC is normalized with the product of mean photon numbers $\left.|\alpha|^{2} \beta\right|^{2}$, a photon number splitting attack can be detected by adding a weak LO beam in the 
decoy-state BB84 protocol to check the CSBC shared between two partites. Intrinsic correlations of the coherent light field have been utilized to implement entanglement, Grover search algorithm, and quantum lithography through different well-designed interference measurement methods [113]. Intrinsic correlations of coherent states do not exhibit nonlocality as a two-photon source. The realization of the intrinsic quantum correlation of a weak coherent state by using the measurement method is a first step toward linear-optics quantum computing with weak light fields and singlephoton source. 



\section{Chapter 4}

\section{Quantum Correlation and Entanglement ${ }^{3}$}

\subsection{Overview}

In this chapter, we discuss the generation of polarization-correlated and polarizationentangled photon pair at telecom wavelength via spontaneous four-waves mixing (SFWM) using a $10 \mathrm{~m}$ long highly nonlinear fiber (HNLF).

We first present an introduction on the development of heralded single photon sources and entangled photon sources along with brief discussion on the merits and drawbacks of different sources. We will review the origin and properties of SFWM as a third order parametric process in optical fiber. The nonlinearity the optical fiber that leads to the parametric process will be discussed, followed by the mechanism of creating a photon pair through the SFWM process. In addition, we will discuss the phase matching condition which is critical for the occurrence of SFWM and explain how to achieve optimum phase matching with the HNLF in our experiment. Next, we explore the origin of spontaneous Raman scattering (SRS) that competing with

\footnotetext{
${ }^{3}$ The material contained in this chapter was previously published in Optics Letters.
} 
SFWM in optical fiber and discuss the methods to minimize and suppress SRS process.

After that, we proceed to give detail description of the single photon detection and coincidence detection system that we built for this work. Single photon detection and coincidence detection is indispensable in characterizing correlated and entangled photon source. We provide a detail illustration on how to precisely detect a single photon by the means of single photon detection and a photon pair by the means of coincidence detection. We also define the coincidence count and accidentalcoincidence count which are important to determine the purity of fiber based photon pair source. Then, we move on to present our experimental setup for generation of correlated and polarization-entangled photon pair. The properties of the HNLF and optical components of the experimental setup will be given in details.

In the experimental results section, first we present the result of single photon count measurement of signal and idler photon. For characterization of correlated photon source, we plot the coincidence count and accidental count as a function of pump photon per pulse with the HNLF at $300 \mathrm{~K}$ and $77 \mathrm{~K}$. Followed by coincidence to accidental-coincidence ratio (CAR) to obtain the optimum pump power for highest CAR value, which is at $7 \times 10^{7}$ photon per pulse ( $430 \mu \mathrm{W}$ in average power) for both temperatures. For characterization of polarization-entangled photon source we measure two-photon interference (TPI) and obtained the visibility of TPI at $300 \mathrm{~K}$ and $77 \mathrm{~K}$. We present the proof for non-locality behavior of polarization-entangled photon pair generated with HNLF by measuring the Clauser-Horne-Shimony-Holt 
(CHSH) Bell's inequality violation of $>12(\approx 5)$ standard deviation at $77 \mathrm{~K}(300 \mathrm{~K})$, respectively.

\subsection{Introduction}

\subsubsection{Heralded Single Photon source}

Over last few decades the developments of single photon source and entangled photon source have experienced explosive growth due to the enormous demand for quantum communication and potential application in quantum information science. For quantum key distribution (QKD), single photon ensures secure communication as eavesdropping on single photon is impossible without exposing the presence of eavesdropper to other [15]. Additionally, single photons are excellent candidates as quantum bits in optical quantum computing [6]. In the case of quantum metrology, elimination of classical noise at single photon level promises noise reduction and precision enhancement of physical measurement [114].

The easiest way for single photon generation is by attenuating a laser pulse to single photon level. However, this method is fundamentally limited due to the Poissonian nature of photon number in the laser beam, where probability of having multiple photons is significant even though the laser is attenuated to average of 1 photon per pulse. In vice versa, when the multi photons effect is suppressed by using a weaker single photon pulse with average photon number of «1, the probability of getting single photon will be extremely low. 
Another approach to produce single photon is by using single quantum emitter as trigger for single photon source through the single radiative transition. However, single quantum emitters such as trapped single atom, molecule, quantum dots, and Nitrogen Vacancy Centers in Diamond diamond require highly complex experimental setup, formidable fabrication technique or cryogenics condition in order to produce single photons [115-117].

Hence, another method of producing single photons by probabilistic generation of correlated photon pair is called into place. This method also known as heralded single photon source as detecting one photon of a photon pair assures the presence of the other photon. To date, generation of photon pair by $\chi^{(2)}$ parametric down conversion in non-linear birefringent crystals and periodically poled lithium niobate (PPLN) waveguides [무, 118]; and $\chi^{(3)}$ spontaneous four-wave mixing (SFWM) in various type of optical fibers, silicon nanowires, chalcogenide $\mathrm{As}_{2} \mathrm{~S}_{3}$ waveguide have been demonstrated [119-121].

\subsubsection{Entangled Photon source}

Generation of probabilistic correlated photon pair lay down the foundation for the creation of entanglement. Ideally, entangled states can be created by combining two correlated photon pair sources. For example, polarization entanglement can be created by combining two indistinguishable, orthogonally polarized correlated photon pair source. The photon pair sources are constructed in such a way that when one photon of a photon pair is detected, the observer unable to predict the 
polarization of the photons but only convinced that the photon pair must be copolarized or anti-polarized.

Initial efforts using two photon emission of cascaded atomic calcium to produce entangled pairs of photons was successfully demonstrated [122]. Amid the success of producing entangled photon pair, it is conceded that many drawbacks exist in these systems. These include complex experiment setup, inconsistent and broad emission angle which severely limit the detection efficiency of the photon pair [123]. Consequently, a vastly improved method of optical parametric down conversion through $\chi^{(2)}$ nonlinearity in crystal is used to convert a pump photon into a pair of photon $[\underline{50}, \underline{58}]$. Although the photon pair production rate using optical parametric down conversion is much higher, stringent phase matching condition affects the photons emission angle and results in multimode emission of photon pair. Thus, necessarily reduces the collection efficiency and limits its application in single mode preferred quantum information processing such as quantum metrology [124].

\subsubsection{Fiber based Correlated and Entangled Photon source}

Correlated and entangled photon-pair sources are essential for implementation of quantum cryptography and quantum key distribution. Particularly, correlated and entangled photon-pair at telecom wavelengths are coincide with the low-loss transmission window $(1.3 \mu \mathrm{m}$ and $1.5 \mu \mathrm{m})$ of the optical fiber. Therefore, have the potential in realizing the global scale QKD through currently available optical fiber networks. 
Direct generation of correlated and entangled photon-pair in optical fiber attracted great interest due to its better spatial mode definition and inherent compatibility with existing fiber optics technologies for long distance transmission, storage and processing. Correlated and entangled photon-pair generation in dispersion-shifted fiber (DSF), and highly nonlinear microstructure fiber (HNMSF) are realized using spontaneous four-waves mixing (SFWM) through $\chi^{(3)}$ nonlinearity $[120, \underline{125}]$. HNMSF has the advantage of high nonlinearity $(\gamma \approx 100 \mathrm{~W} / \mathrm{km})$ due to its much smaller core size, therefore required shorter interaction length, where $\gamma$ is third order nonlinear coefficient of a medium. However, smaller core size, asymmetric mode profile and inhomogeneous microstructure limit its compatibility with single mode fiber. In contrast, DSF is compatible with standard optical fiber, even though has much lower nonlinearity $(\gamma \approx 2 \mathrm{~W} / \mathrm{km})$. Therefore, several hundred meters of interaction length is needed for photon pair generation in DSF.

In this work, we generate correlated and entangled photon-pair using a short dispersion shifted, highly nonlinear fiber (HNLF), which has advantage of high nonlinearity and yet highly compatible with the standard optical fiber. Hence, HNLF could be an outstanding candidate for fiber based correlated and entangled photon pair source at telecom wavelengths. 


\subsection{Spontaneous Four-wave Mixing}

In this section, we review the origin and mechanism of spontaneous four-wave mixing (SFWM) in optical fiber to present a clear physical picture on this phenomenon.

SFWM process in optical fiber is a parametric process involving nonlinear interaction of four optical waves due to the third-order order susceptibility of a material [126]. Parametric process originates from the nonlinear response of bound electrons in the interaction medium to an applied optical field. Nonlinear polarization of the bound electrons that induced by the applied optical field is dependent to the nonlinear susceptibilities of the material. In optical fiber, second order susceptibility $\chi^{(2)}$ vanishes due to the isotropic nature of silica glass [127]. In contrast, third order susceptibility $\chi^{(3)}$ or Kerr nonlinearity is the dominant nonlinearity that leads to thirdorder parametric processes in optical fiber including SFWM [128].

When an intense electromagnetic field propagating in an optical fiber, the induced polarization can be described by using wave equation derived from of Maxwell's equations, given as:

$$
\nabla^{2} \mathbf{E}(\mathbf{r}, \mathrm{t})-\frac{1}{\mathrm{c}^{2}} \frac{\delta \mathbf{E}}{\delta \mathrm{t}^{2}}=\mu_{0} \frac{\delta \mathbf{P}_{\mathbf{L}}}{\delta \mathrm{t}^{2}}+\mu_{0} \frac{\delta \mathbf{P}_{\mathrm{NL}}}{\delta \mathrm{t}^{2}}
$$

Where $\mathbf{E}(\mathbf{r}, \mathbf{t})$ is the electromagnetic field, $\mu_{0}$ is the linear vacuum permeability, and $\mathrm{c}$ is the speed of light in vacuum. The induced linear polarization is given by,

$$
\mathbf{P}_{\mathrm{L}}=\varepsilon_{0} \chi^{(1)} \cdot \mathbf{E}(\mathbf{r}, \mathrm{t}) .
$$


The induced third-order nonlinear polarization is given by,

$$
\mathbf{P}_{\mathbf{N L}}=\varepsilon_{0} \chi^{(3)} \cdot \mathbf{E}(\mathbf{r}, \mathrm{t}) \mathbf{E}(\mathbf{r}, \mathrm{t}) \mathbf{E}(\mathbf{r}, \mathrm{t}),
$$

with $\varepsilon_{0}$ is the permittivity in vacuum.

Consider four electromagnetic fields, with optical frequencies $\omega_{1}, \omega_{2}, \omega_{3}$, and $\omega_{4}$ that are copolarized in x-axis involved in the SFWM process, the total electric field propagating in the optical fiber is given as,

$$
\mathbf{E}(\mathbf{r}, \mathrm{t})=\frac{1}{2} \hat{\mathrm{x}} \sum_{j=1}^{4} E_{j} \exp \left[i\left(k_{j} z-\omega_{j} t\right)\right]+c . c,
$$

where the $k_{j}=\frac{n_{j} \omega_{j}}{c}, n_{j}$ is the refractive index of the propagating medium at frequency $\omega_{j}$ for the $j$-th electromagnetic field $E_{j}$ and $c . c$ stands for complex conjugate.

Using Eq. 4.4, the induced third-order nonlinear polarization vector can be expressed as

$$
\mathbf{P}_{\mathrm{NL}}=\frac{1}{2} \hat{\mathrm{x}} \sum_{j=1}^{4} P_{j} \exp \left[i\left(k_{j} z-\omega_{j} t\right)\right]+c . c .
$$

Where $P_{j}$ is the nonlinear polarization induced by electromagnetic field $E_{j}$. For instance, the third-order nonlinear polarization for the electromagnetic field $\omega_{4}$, can be written as,

$$
\begin{aligned}
P_{4} & =\frac{3 \varepsilon_{0}}{4} \chi^{(3)}\left\{\left[\left|\mathrm{E}_{4}\right|^{2}+2\left(\left|\mathrm{E}_{1}\right|^{2}+\left|\mathrm{E}_{2}\right|^{2}+\left|\mathrm{E}_{3}\right|^{2}\right)\right] \mathrm{E}_{4}+2 \mathrm{E}_{1} \mathrm{E}_{2} \mathrm{E}_{3} \exp \left(i \theta_{+}\right)+\right. \\
& \left.2 \mathrm{E}_{1} \mathrm{E}_{2} \mathrm{E}_{3} \exp \left(i \theta_{-}\right)\right\}
\end{aligned}
$$


where

$$
\begin{aligned}
& \theta_{-}=\left[k_{1}+k_{2}+k_{3}-k_{4}\right] z-\left[\omega_{1}+\omega_{2}+\omega_{3}-\omega_{4}\right] t \\
& \theta_{+}=\left[k_{1}+k_{2}-k_{3}-k_{4}\right] z-\left[\omega_{1}+\omega_{2}-\omega_{3}-\omega_{4}\right] t .
\end{aligned}
$$

The term proportional to $\mathrm{E}_{4}$ in Eq. 4.6 is corresponding to other nonlinear processes such as self-phase modulation, and cross-phase modulation [126, 129]. The term containing $\theta_{-}$is responsible for the third harmonic generation or frequency conversion. The term involving $\theta_{+}$is responsible for SFWM.

Effective parametric coupling of SFWM process requires $\theta_{+}=0$ for all $P_{j}$ for $j=1-4$, where $\theta_{+}$is identical for all $P_{j}$. Accordingly, effective SFWM takes place if the following conditions are satisfied

$$
\begin{gathered}
k_{1}+k_{2}=k_{3}+k_{4}, \\
\omega_{1}+\omega_{2}=\omega_{3}+\omega_{4} .
\end{gathered}
$$

Eq. 4.9 is the matching of the wave vectors, which is the well-known phase matching condition, while Eq. (4.10) reflects the requirement of specific choice of frequencies in SFWM process, which is the energy conservation requirement.

In quantum-mechanical term, Eq. (4.10) means that two photons at $\omega_{1}$ and $\omega_{2}$ are annihilated and two photons at $\omega_{3}$ and $\omega_{4}$ are created in SFWM process. The output photon at higher frequency $\left(\omega_{4}\right)$ is signal photon and output photon at lower frequency $\left(\omega_{3}\right)$ is idler photon. Eq. 4.10 can be fulfilled by careful selection of the frequencies $\omega_{3}$ and $\omega_{4}$. In the case of degenerate SFWM where $\omega_{1}=\omega_{2}$, two 
photons at $\omega_{1}$ will be annihilated and creates two photons located symmetrically in frequency space at $\omega_{3}$ and $\omega_{4}$. The frequency shift $\Omega$ is given by

$$
\Omega=\omega_{1}-\omega_{3}=\omega_{4}-\omega_{1}
$$

as illustrated in Fig. 4.1.

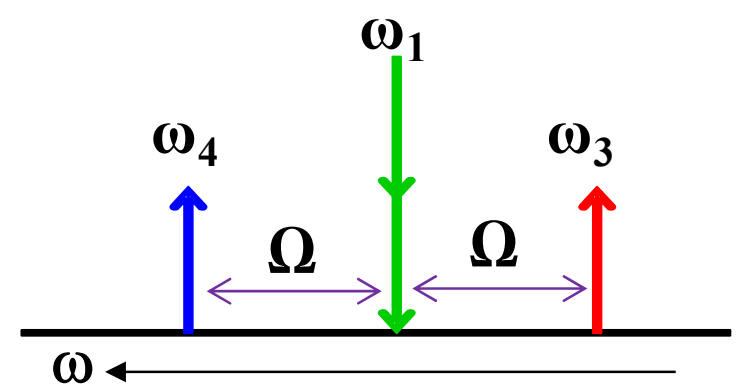

Figure 4.1. Illustration of degenerate Spontaneous four wave mixing process where two photons at $\omega_{1}$ are annihilated and two photons at $\omega_{3}$ and $\omega_{4}$ are created.

The optimum phase matching condition in Eq. 4.9 can be rearranged and written as $\Delta k=0$, where the net wavevector mismatch $\Delta k$ is given by

$$
\Delta k=k_{3}+k_{4}-k_{1}-k_{2}
$$

The net wavevector mismatch consists of the phase mismatch resulting from the summation of material dispersion $\Delta k_{M}$, waveguide dispersion $\Delta k_{W}$, and the nonlinear effects $\Delta k_{N L}[\underline{126}]$,

$$
\Delta k=\Delta k_{M}+\Delta k_{W}+\Delta k_{N L}
$$

Where, the three contributions in Eq. (4.13) are given as [126], 


$$
\begin{aligned}
& \Delta k_{M}=n_{3} \omega_{3}+n_{4} \omega_{4}-2 n_{1} \omega_{1}, \\
& \Delta k_{W}=\left[\Delta n_{3} \omega_{3}+\Delta n_{4} \omega_{4}-\left(\Delta n_{1}+\Delta n_{2}\right) \omega_{1}\right] / c, \\
& \Delta k_{N L}=\gamma\left(P_{1}+P_{2}\right) .
\end{aligned}
$$

$\gamma$ is the nonlinear coefficient of propagating medium and $\Delta n_{j}$ is the change in the refractive index due to waveguiding. One of these three contributions should be negative in order to achieve the phase matching condition $\Delta k=0$.

In the single mode fiber, the contribution of the $\Delta k_{W}$ to the net wavevector mismatch, $\Delta k$ is negligible as $\Delta n_{j}$ is similar for all waves in SFWM process. $\Delta k_{N L}$ in Eq. 4.13 is always positive as $\gamma, P_{1}$, and $P_{2}\left(P_{1}=P_{2}\right.$ for partially degenerate SFWM ) are all positive. The negative dispersion is attained from the material dispersion $\Delta k_{M}$ by using the pump wavelength larger than the zero dispersion wavelength of the fiber where $\lambda_{1}=\lambda_{2}>\lambda_{D}$ [126]. Ideally, the pump wavelength is selected in vicinity to the $\lambda_{D}$ so that the magnitude of the $\Delta k_{M}$ is very small and can be compensated by adjusting the pump power $P_{1}$ and $P_{2}$ to achieve optimized phase matching $\Delta k=0$.

In next section, we discuss the undesirable Spontaneous Raman Scattering, which is the competing phenomenon to SFWM. 


\subsection{Spontaneous Raman scattering}

Spontaneous Raman Scattering (SRS) occurs simultaneously with SFWM process in HLNF as the pump photons for the SFWM will also work as pump photons for SRS [126]. In SRS process, an incident photon propagating in a medium scattered by a molecule to produce a lower or higher energy photon, with the molecule makes transition between vibration states. In contrast to the SFWM process, the energy of the photon involved in SRS is not conserved as it gains or losses energy in the scattering process. The photon can be shifted to higher frequency (energy) $\omega_{a s}$ as anti-Stokes Raman photon or shifted to lower frequency (energy) $\omega_{s}$ as Stokes Raman photon given as

$$
\begin{aligned}
& \omega_{s}=\omega_{o}-\Omega \\
& \omega_{a s}=\omega_{o}+\Omega
\end{aligned}
$$

where $\Omega$ is the frequency shift which is dependent on the molecule vibrational states. The energy diagram of SRS is shown in Fig. 4.2.

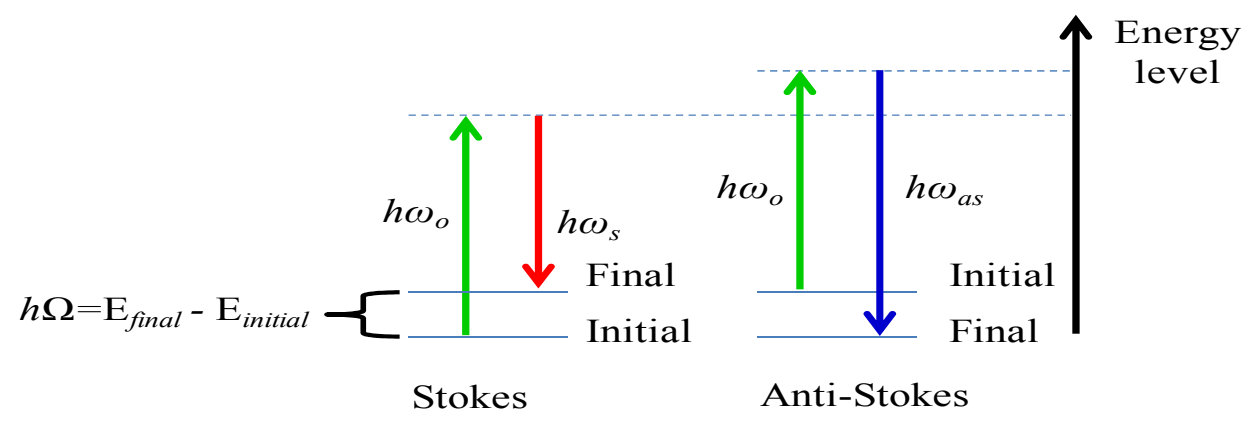

Figure 4.2. Energy level diagram of Stokes and anti-Stokes spontaneous Raman scattering 
Raman spectrum of silica fiber can be coincides with the signal and idler channels of photon-pairs generated by SFWM [130]. The presence of Stokes and anti-Stokes Raman photons induce undesirable accidental coincidences and deteriorate the correlation characteristics of the photon-pair source. As the Raman photons threaten the purity of fiber based photon-pair source, considerable efforts have been devoted to reduce the Raman photons. One of the approaches is using microstructure fiber to generate photon-pair widely separated in wavelength beyond the Raman scattering spectrum [125]. However, this option is less appealing as the photon-pair generated by this method is not in the low-loss transmission bandwidth of optical fiber. Another feasible option to reduce the Raman photon is by selecting the detection bands of signal and idler photon-pair closely to the pump wavelength where the Raman scattering is less probable [130]. Moreover, the Raman photon can be suppressed by reducing the temperature of the fiber [131]. Stokes and anti-Stokes Raman photons are proportional to thermal population factors $n_{t h}+1$ and $n_{\text {th }}$ [132]. The thermal population factor is given as,

$$
n_{t h}=\frac{1}{\exp \left(\frac{h c \Delta \lambda}{k_{B} T \lambda^{2}}\right)-1}
$$

where $\Delta \lambda$ is the detuning from the pump wavelength, $\lambda$ is the pump wavelength, $T$ is the HNLF's absolute temperature in Kelvin, $c$ is speed of light, $h$ is Planck's constant, and $k_{B}$ is Boltzmann's constant. Given that $\Delta \lambda=6.5 \mathrm{~nm}$, theoretical calculation of thermal population factor for Stokes (anti-Stokes) Raman photons is 
about $8.2(7.2)$ at $300 \mathrm{~K}$ and $2.5(1.5)$ at $77 \mathrm{~K}$. Hence, Raman photons are expected to be reduced as we cool the fiber from $300 \mathrm{~K}$ to $77 \mathrm{~K}$ in our experiment.

\subsection{Detection Apparatus}

\subsubsection{Single Photon detection}

Single photon detection is crucial in this work. Without appropriate single photon detectors, we will not be able to characterize the photon pair source. Signal and idler photon-pairs are detected by fiber coupled $\mathrm{InGaAs} / \mathrm{InP}$ avalanche photodiodes operated in gated Geiger mode at room temperature. The APDs were reversed biased with $64 \mathrm{~V}$ DC voltage and gated by $1 \mathrm{~ns}$ full width half maximum (FWHM) gate pulses with $10 \mathrm{~V}$ in amplitude at the rate of $726 \mathrm{kHz}$. The gate pulses were triggered and 1/64 frequency-divided from the mode-locked pump laser pulses. For the detection of single photons, gated photon counter was synchronized by electrical gate pulses that coincide with incoming single photons. The timing of gate pulses for each APD was independently adjusted by a multi-channels digital delay generator and coincides with the arrival of the signal and idler photons at the APDs as illustrated in Fig. 4.3. 


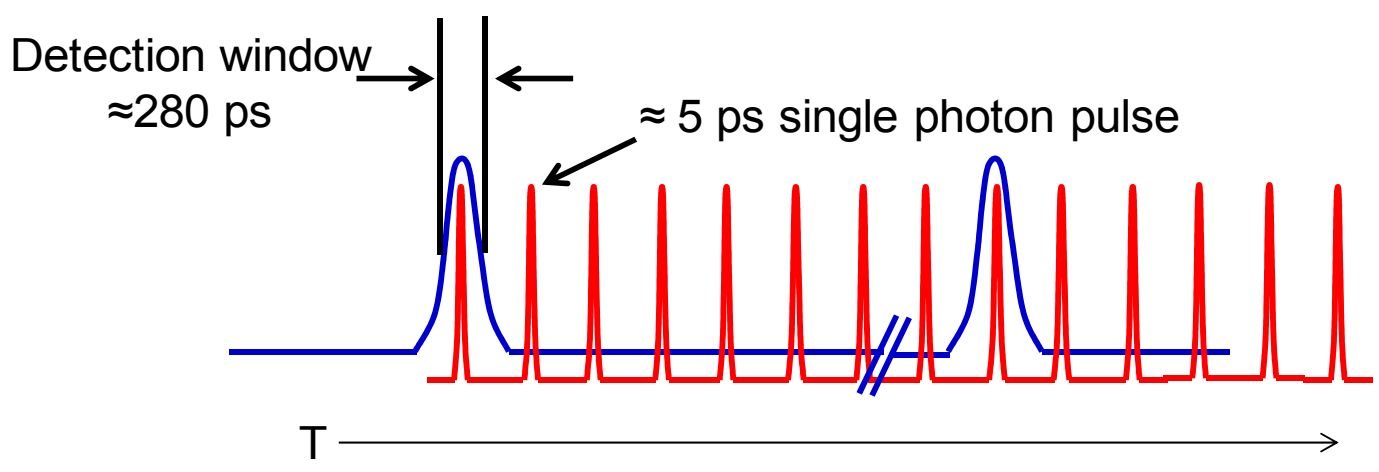

Figure 4.3. Illustration of electrical gate pulses precisely coincides with incoming single photon pulses for the single photon detection.

In Fig. 4.4, we show a plot of the quantum efficiency versus the dark count probability for the two single photon detectors that we built. Dark count probability is defined as the probability of the single photon detector to register a count when no photon arrived at the detector.

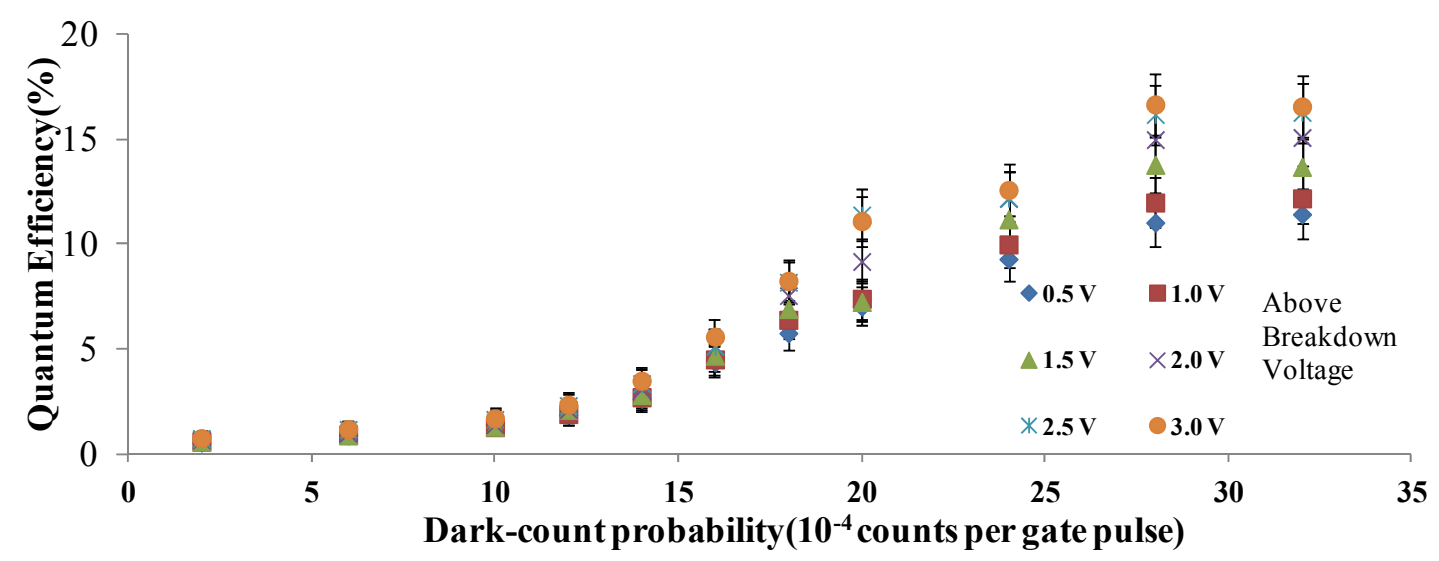

Figure 4.4. Plot of quantum efficiency versus the dark count probability of single photon detector at different biased voltages above the breakdown voltage. 
For the single photon detector to operate with sufficient stability in optimum condition; the quantum efficiency, dark counts probability, and FWHM detection window of APD1 (APD2) were about 10.3\% (9.8\%), 2.5 $\times 10^{-3}\left(2.2 \times 10^{-3}\right)$ and $280 \mathrm{ps}$ (250 ps). The dual channel gated photon counter was used to register single photon count from each APD separately. The electrical pulses from the output of the APDs were fed to the photon counter, where its gate timing system was triggered by the same 1/64 down counter circuit. The photon counter always received the electrical pulses from the APDs. The discriminator were set at a level to to distinguish the "avalanche pulse" (single photon detected) and "no avalanche pulse" (no photon detected). A single photon count was registered by the photon counter with the detection of an "avalanche pulse" on each APDs. Both single photon detectors and photon counter were triggered by the same pump laser used to generate photon pair, thus eliminating the possible phase jittering between optical pulse and detection system.

\subsubsection{Coincidence detection}

Every single photon count registered in each channel (A and B) of the photon counter independently triggered an electrical pulse which was transmitted to the multichannel scaler for the coincidence detection. The electrical pulse from one of the channel (A) in photon counter was used as trigger input and electrical pulse from channel (B) was used for signal input for the scaler. A delay pulse generator was used to delay the electrical pulse of channel (B). The time delay was set to match the rise time of the 
detection circuit in the scaler after a trigger. At each trigger, the scaler registered signal pulses at different arrival time for a preset total time bin and accumulated a histogram of detection events over an integration time. A typical histogram obtained in our experiment is depicted in Fig. 4.5.

The coincidence count recorded from the same gated time interval can be distinguished as the time bin with the highest number of recorded count. The rest of the time bins are accidental coincidence counts which arise from the coincidence detection between adjacent pulses.

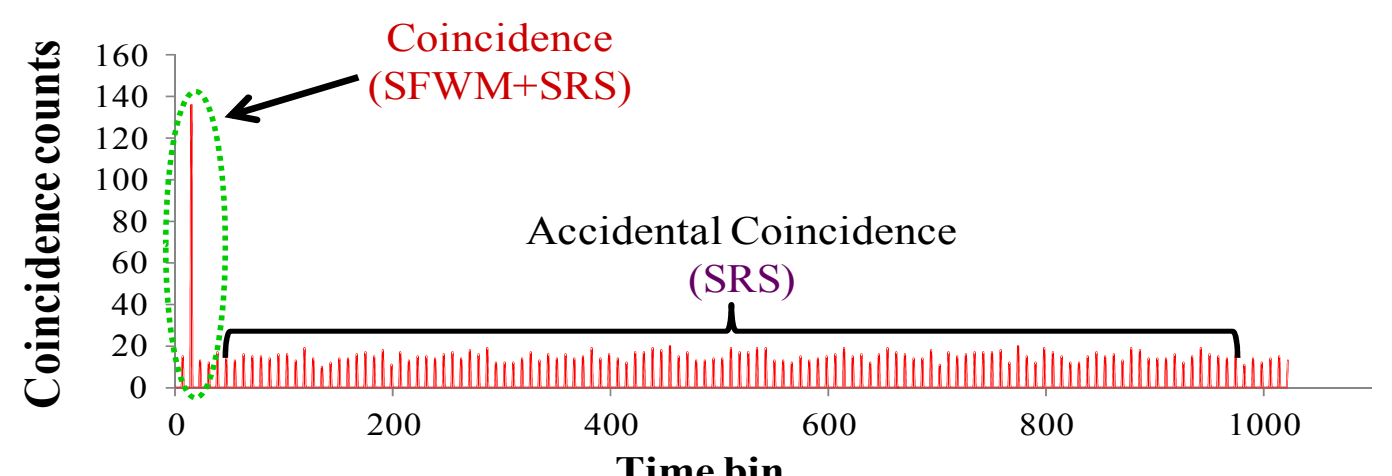

Figure 4.5. A typical histogram acquired by the multichannel scaler. The time bin with highest counts is the Coincidence count, and the rest of the bins are each accidental coincidence count.

A coincidence count was recorded when both APDs detected a photon at the same gated time interval, while an accidental coincidence count was recorded when both APDs detected a photon at the adjacent gated time interval as illustrated in Fig. 4.6. Correlated photon-pair from SFWM, un-correlated noise photon from Raman 
scattering and detector dark count all contributed to the coincidence count. Accidental coincidence count was attributed to un-correlated noise photon from Raman scattering and detector dark count. Information of both coincidence count and accidental coincidence count were indispensable for characterization the purity of the correlated photon source in our experiment.

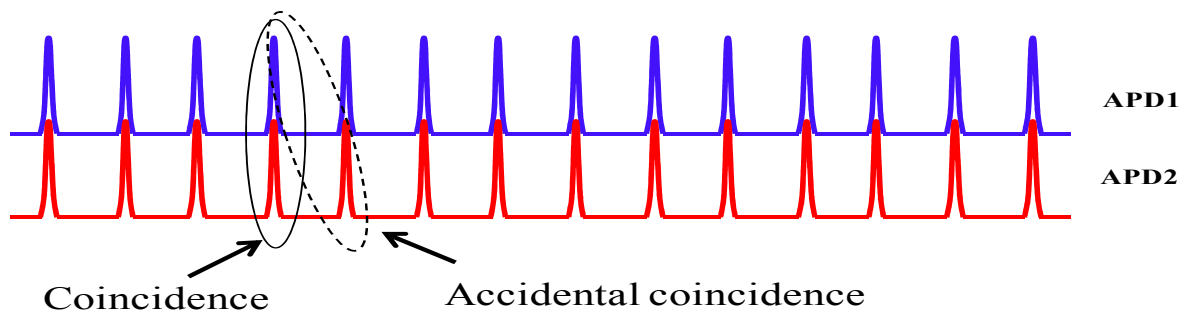

Figure 4.6. Diagram shows timing correlation of coincidence count and accidentalcoincidence count from the pulse sequences in APD1 for idler photon and APD2 for signal photon.

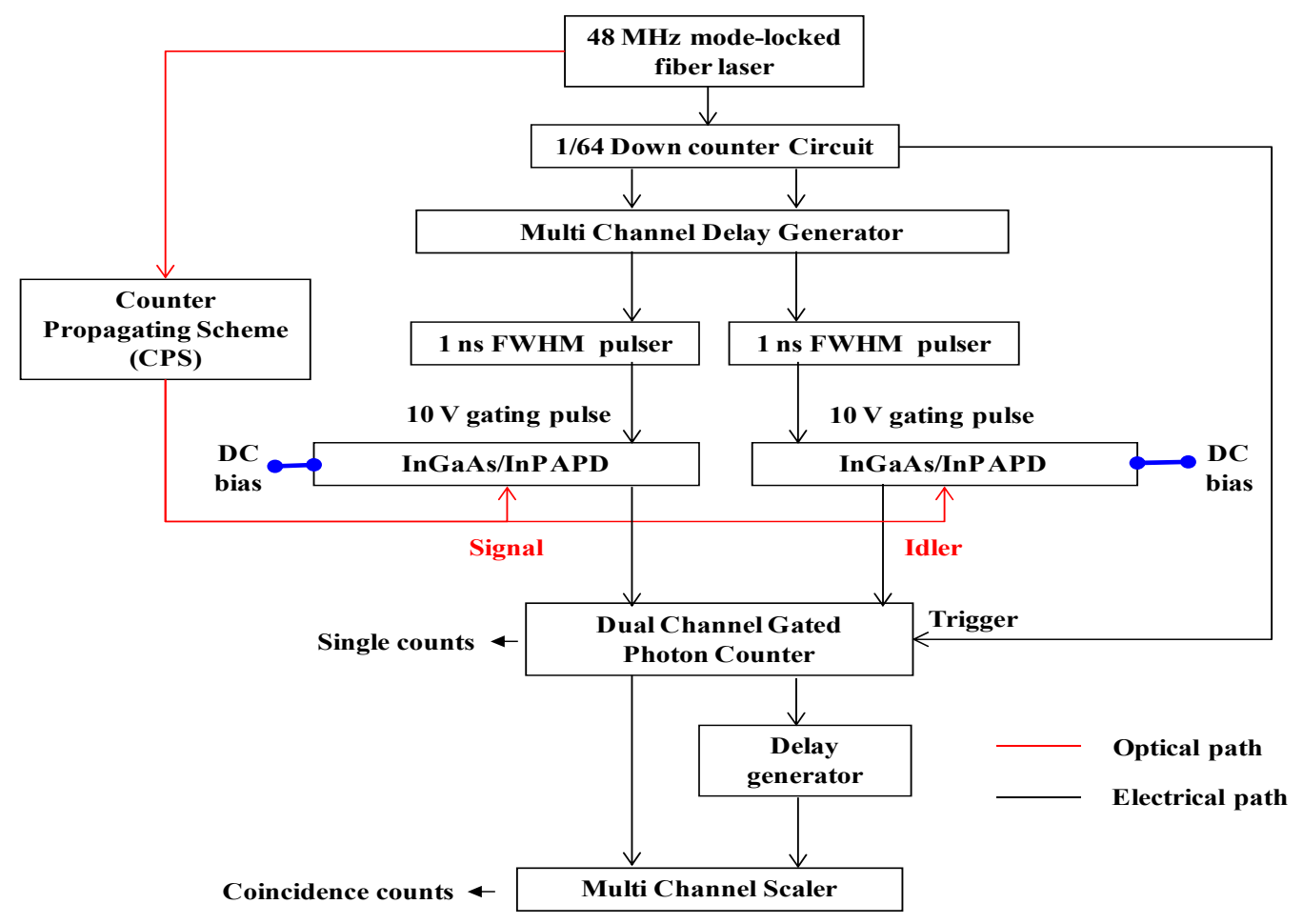

Figure 4.7. Schematic Layout of single photon detection and coincidence detection system 
A complete schematic layout of single photon detection and coincidence detection system that was setup in this work is shown in Fig. 4.7.

\subsection{Experiment Setup}

We use a $10 \mathrm{~m}$ long HNLF, fabricated by Sumitomo as a nonlinear interaction medium for the photon pair generation [133]. The HNLF with the core diameter of $4 \mu \mathrm{m}$ was fusion spliced to a single mode fiber for a measured total loss of less than $1 \mathrm{~dB}$. It has excellent compatibility with standard transmission fiber. The HNLF's zero dispersion wavelength was engineered at $1554 \mathrm{~nm}$, and has a high nonlinear coefficient $\gamma$ of $30 \mathrm{~W} / \mathrm{km}$, attenuation of $0.9 \mathrm{~dB} / \mathrm{km}$, and effective area of $8.5 \mu \mathrm{m}^{2}$. The $\gamma$ value of this HNLF is significantly higher than both the of conventional nonlinear fiber $(\approx 10 \mathrm{~W} / \mathrm{km})$ and dispersion-shifted fiber $(\approx 2 \mathrm{~W} / \mathrm{km})[130]$. The characteristic data sheet of the HNLF is attached in Appendix F.

The pump pulse at $1554.1 \mathrm{~nm}(\mathrm{FWHM}$ spectral width $\approx 0.8 \mathrm{~nm}$, pulse duration $\approx 5$ ps and repetition rate of $46.5 \mathrm{MHz}$ ) was spectrally carved out from a mode-locked femtosecond fiber laser by using a Dense Wavelength Division Multiplexer (DWDM) filter with $1 \mathrm{~nm}$ bandwidth and was further amplified using an Erbium-doped-fiber-amplifier. The amplified spontaneous emission from the Erbium-doped-fiber-amplifier was suppressed by utilizing two cascaded DWDM filters. The suppression of the amplified spontaneous emission is critical for our experiment. The residual spontaneous emission of EDFA that coincides with signal-idler wavelengths will add to the accidental coincidence count and prohibit accurate characterization of the photon-pair source. The amplified pump pulses were 
launched via fiber-to-free space collimators and go through a quarter wave plate (QWP) and a half wave plate (HWP) to compensate changes of polarization states of pump pulses caused by the fiber birefringence. The combination of the HWP, QWP and the PBS1 produced a horizontally polarized pump pulse. Both the correlated photon pair and entangled photon pair could be generated using the same experimental setup by manipulating the polarization angle of the of linearly polarized pump pulses.

For the generation of correlated photon-pair, the HWP1 in front of the PBS2 was set at $0^{\circ}$ to maintain pump pulse in horizontal polarization. Horizontally polarized pump pulses propagated through the HNLF in clockwise direction, and emerged at the output port of the PBS2 along with the probabilistically generated co-polarized signal and idler photon-pairs .

For the generation of the polarization entangled photon, HWP1 was oriented at $22.5^{\circ}$ to project pump pulse at $45^{\circ}$ polarization angle. After passing through the PBS2, the pump pulse was divided equally into horizontally and vertically polarized components. Both pump pulses propagated through the HNLF in clockwise and counterclockwise directions, respectively. This configuration for generation polarization entangled state is also known as counter propagating scheme (CPS). Each component of the polarized pump pulse probabilistically generated its own signal-idler photon pair $\left(H_{s} H_{i}\right.$ and $\left.V_{s} V_{i}\right)$ respectively. The photon pairs were created via SFWM process and emerged from the same output port of the PBS2. The PBS2 is the principal component in CPS as it allows the two orthogonally polarized pump pulses to propagate in a common path. The signal-idler photon pairs superimposed at the output to generate Bell's (polarization entangled) state $\left|\Psi^{+}\right\rangle=$ $\frac{1}{\sqrt{2}}\left[\left|H_{i} H_{s}\right\rangle+e^{-2 i \phi_{p}}\left|V_{i} V_{S}\right\rangle\right]$, where $\phi_{p}$ is the relative phase between the horizontally and 
vertically polarized pump pulses. In addition, half of the un-polarized Raman noise photons were blocked from exiting the output of the PBS, hence improve the purity of the entangled photon pair. The common path nature of CPS and low polarization dispersion $(-0.1 \mathrm{ps} / \mathrm{nm}-$ $\mathrm{km}$ ) in a $10 \mathrm{~m}$ HNLF established the timing indistinguishability, and phase stability between the horizontally and vertically polarized signal-idler photon pairs at the output of the PBS. In addition to $\left|\Psi^{+}\right\rangle=\frac{1}{\sqrt{2}}\left[\left|H_{i} H_{S}\right\rangle+\left|V_{i} V_{S}\right\rangle\right]$, CPS was employed to produce all other Bell states. Bell state $\left|\Psi^{-}\right\rangle=\frac{1}{\sqrt{2}}\left[\left|H_{i} H_{S}\right\rangle-\left|V_{i} V_{S}\right\rangle\right]$ was achieved by inserting a QWP at $0^{\circ}$ after the HWP1 to adjust the relative phase between two pump pulses to $\phi_{p}=90^{\circ}$. While Bell states $\left|\Phi^{+}\right\rangle=\frac{1}{\sqrt{2}}\left[\left|H_{i} V_{S}\right\rangle+\left|V_{i} H_{s}\right\rangle\right]$ and $\left|\Phi^{-}\right\rangle=\frac{1}{\sqrt{2}}\left[\left|H_{i} V_{s}\right\rangle-\left|V_{i} H_{s}\right\rangle\right]$ were obtained by adding a HWP at $45^{\circ}$ in signal channel of the configurations for the aforementioned $\Psi^{ \pm}$Bell states.

The quarter wave plate and half wave plate in the CPS were used to compensate for the birefringence induced polarization changes of signal-idler. In order to measure the signal-idler photon-pair, remaining pump photons must be blocked to prevent them from reaching APDs. Since only about 0.1 photon is scattered by a typical 5-ps-duration pump pulse that contains approximately $10^{8}$ photons, a pump photon rejection ratio in excess of $100 \mathrm{~dB}$ is required. Hence, DWDM filters with $1 \mathrm{~nm}$ FWHM bandwidth at $1560.6 \mathrm{~nm}$ and $1547.7 \mathrm{~nm}$ were used to separate the signal and idler photons with $6.5 \mathrm{~nm}$ detuning from the pump wavelength. The DWDM filters were used to suppress the pump pulse with isolation of more than $110 \mathrm{~dB}[\underline{130}, \underline{134}]$. 


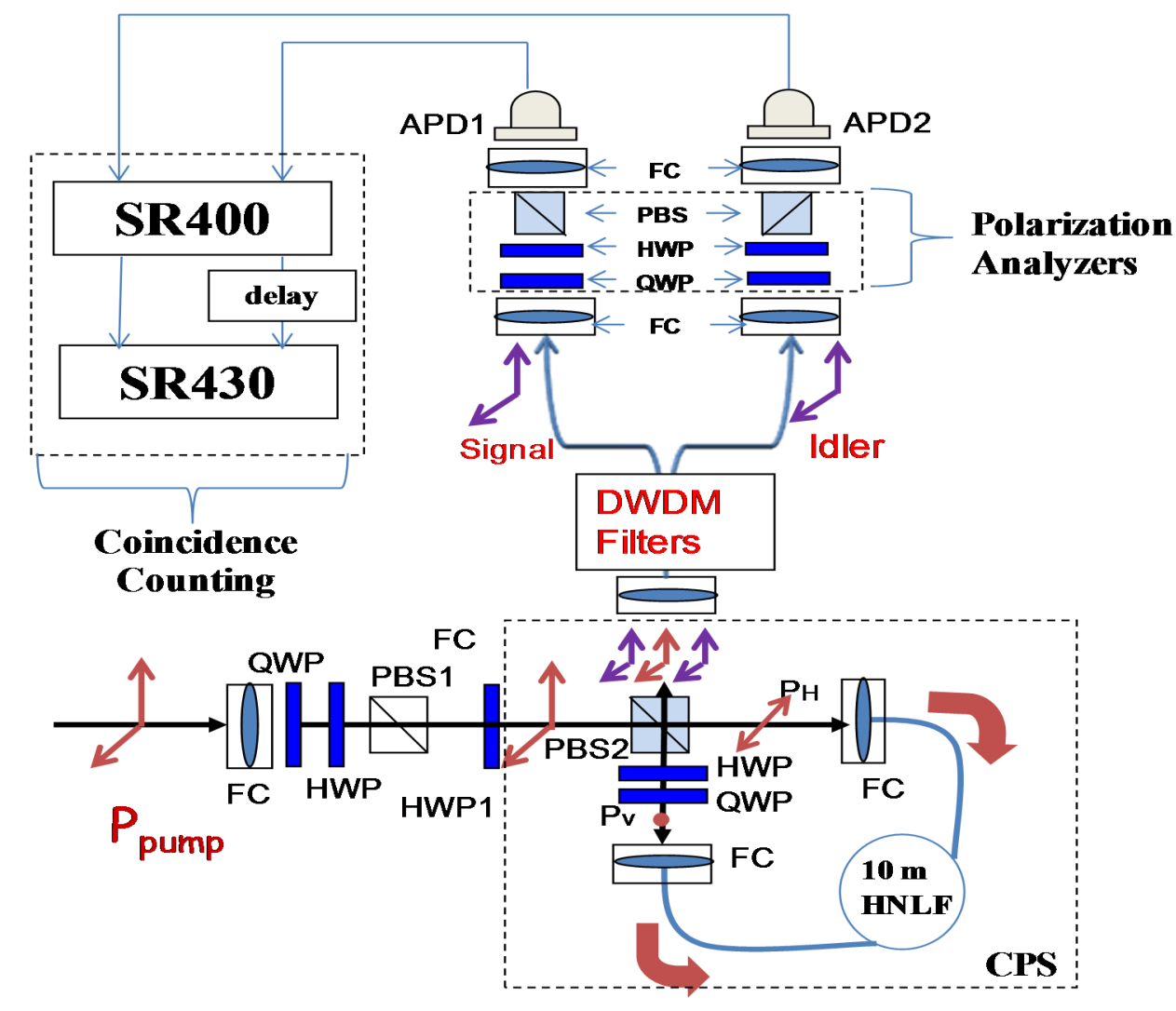

Figure 4.8. Layout of experiment setup. FC (fiber-to-free space collimators); PBS (polarization beam splitter); HWP and QWP (half- and quarter-wave plates); DWDM (dense wavelength division multiplexer); APD (Avalanche photodiode).

The transmission spectrum of the signal and idler photon is shown in Fig. 4.9. The rejection ratio of about $100 \mathrm{~dB}$ was obtained with the cascaded DWDM filter. The total insertion losses of the cascaded DWDM filters for signal and idler were measured to be about $0.9 \mathrm{~dB}$. The selection of signal and idler wavelengths at small detuning $(6.5 \mathrm{~nm})$ from the pump wavelength was chosen to minimize the copolarized Raman scattering photons. However, the selection of signal and idler wavelengths too close to pump wavelength was not permitted. This is due to the 
broadening of pump pulses, which commenced from the self-phase modulation as its traveled along the HNLF [129].

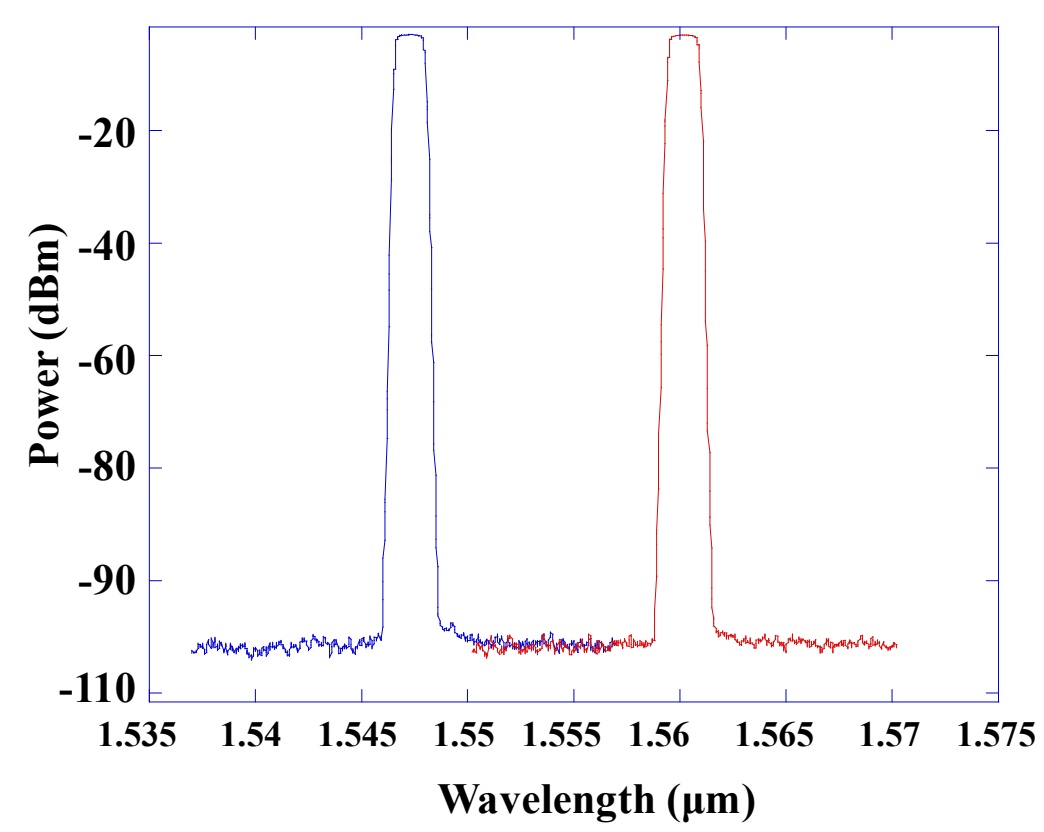

Figure 4.9. Transmission spectrum of cascaded DWDM filters for signal (1547.4 nm) and idler channel $(1560.6 \mathrm{~nm})$

The outputs photons from the signal and idler channels were guided through the polarization analyzers consisting of a quarter-wave plate, a half-wave plate and a polarizing beam splitter. Note that the polarizing beam splitter in the polarization analyzers further suppressed the remained cross-polarized scattered Raman photons. However, the copolarized Raman photons still passed through polarizing beam splitter and reached the detectors. Signal and idler photons were detected by single photon detection and coincidence detection system that was described in previous section. Total detection efficiencies of signal and idler photon were about $4.99 \%$ and $4.87 \%$ respectively. The detection efficiencies included propagation losses of optical components in CPS and 
polarization analyzer, splicing losses of the HNLF and the APDs quantum efficiencies. We will present the experimental results for the characterization of correlated and entangled photon source using HNLF next.

\subsection{Results}

\subsubsection{Single Photons Count}

We generated correlated photon-pair and measured the single photon count per pulse for both signal $(1547.7 \mathrm{~nm})$ and idler $(1560.6 \mathrm{~nm})$ channel as a function of number of pump photon per pulse at two temperatures. With the highly non-linear fiber in room temperature $(300 \mathrm{~K})$ and when it is cooled to liquid nitrogen temperature $(77 \mathrm{~K})$. The HNLF in plastic buffer coating was cooled to $77 \mathrm{~K}$ by immersing it into liquid nitrogen. We observed advancement of photons arrival times by about $130 \mathrm{ps,}$ indicated contraction in fiber length when the HNLF was cooled to $77 \mathrm{~K}$. It is also noted that the zero dispersion wavelength of the HNLF was shifted toward shorter wavelengths at $77 \mathrm{~K}$. The polarization analyzers were adjusted in such a way that horizontally polarized signal and idler photons will pass to APD1 and APD2.

The single photon count per pulse for signal and idler is proportional to the number of pump photon $\left(\boldsymbol{N}_{\text {pump }}\right)$, and is given by,

$$
N_{s(i)} \propto S_{1} N_{\text {pump }}+S_{2} N_{\text {pump }}{ }^{2} .
$$

Where $\boldsymbol{S}_{1}$ is the coefficient of the Raman scattering and $\boldsymbol{S}_{2}$ is the coefficient of the four wave mixing process. The measurement of single photon count per pulse, for 
both signal and idler channel as a function of number of pump photon per pulse are depicted in Fig. 4.10 and Fig. 4.11. The dashed lines are the curve fitting using Eq. 4.17. In the data presented in Fig. 4.10 and Fig. 4.11, the intrinsic detector dark count was subtracted from the single count measurement, thus every count registered indicates the detection of FWM photon or SRS photon. The difference in single photon counts per pulse between the signal and idler photon are mainly due to the unequal detection efficiencies for signal (4.99\%) and idler channel (4.87\%) in our experiment setup.

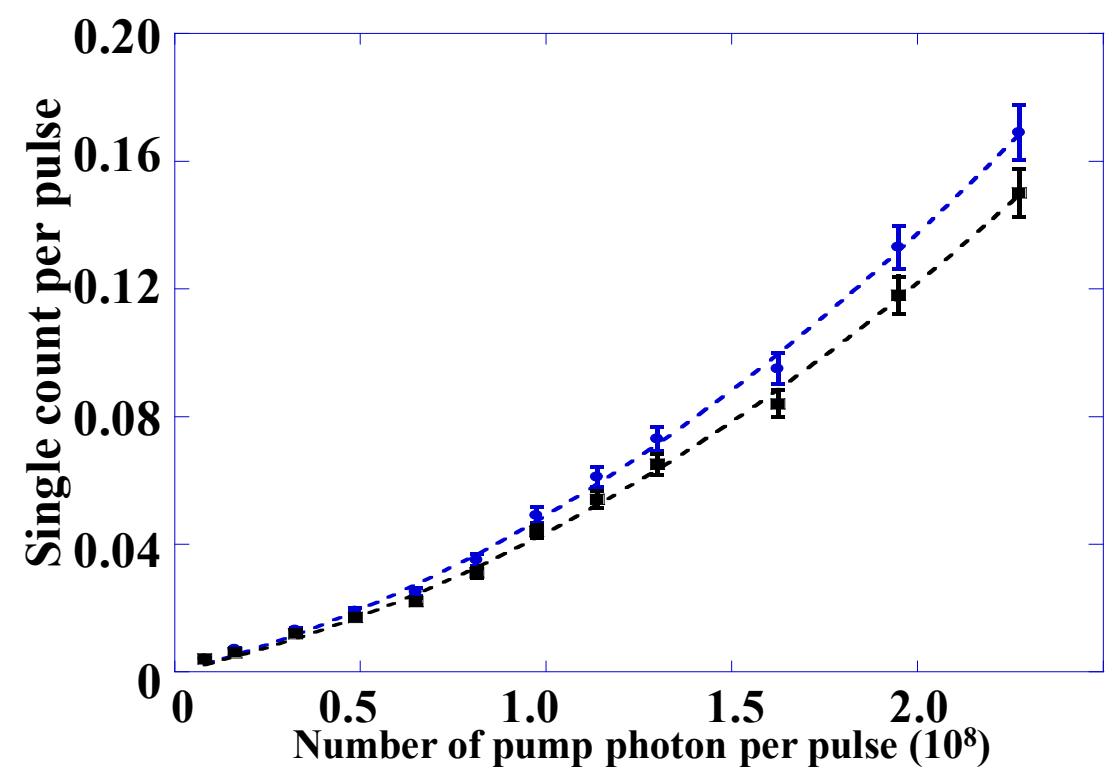

Figure 4.10. A graph showing single photon counts per pulse of Signal (Blue dot) and Idler (Black square) photon as a function of number of pump photon per pulse when the HNLF is in room temperature $(300 \mathrm{~K})$. 


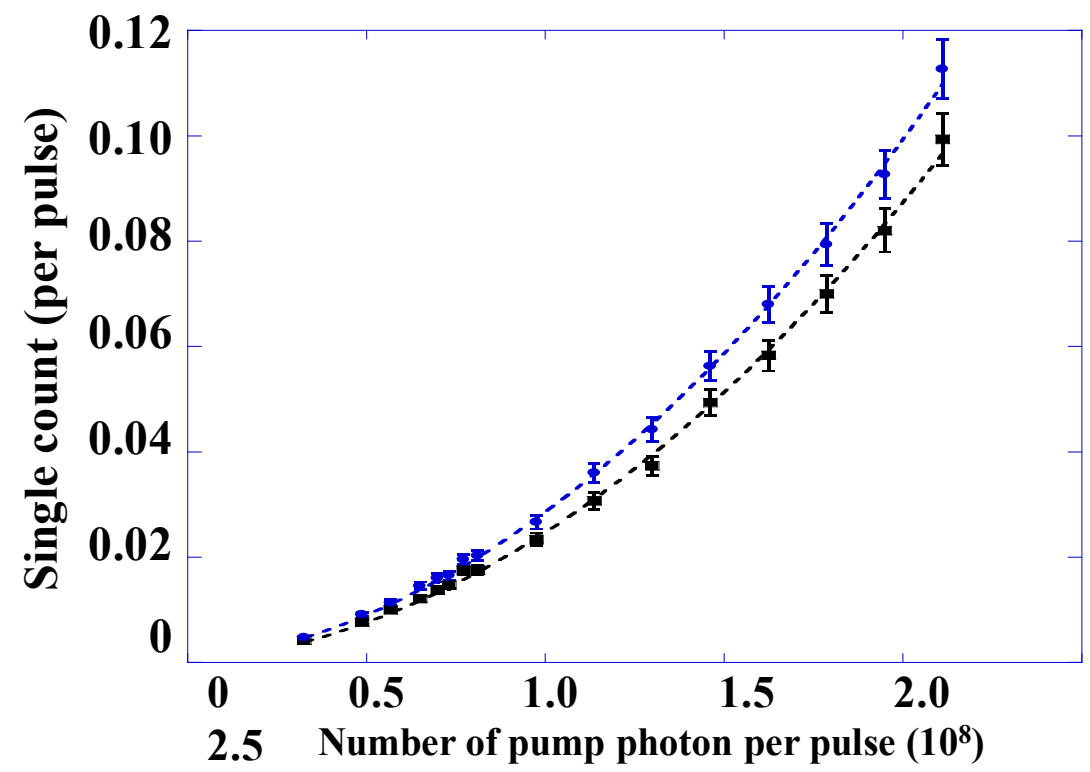

Figure 4.11. A graph showing single photon counts (per pulse) of Signal (Blue dot) and Idler (Black square) photon as a function of number of pump photon per pulse when the HNLF is cooled to $77 \mathrm{~K}$ by immersing in liquid nitrogen.

Comparing the plots in Fig. 4.10 and 4.11 for the HNLF in $300 \mathrm{~K}$ and $77 \mathrm{~K}$, one can see that single photon count for both signal and idler decreased when the HNLF was cooled to $77 \mathrm{~K}$. The decrease in single photon count at $77 \mathrm{~K}$ was mainly due to suppression of Raman photons at $77 \mathrm{~K}$. In addition, the propagation loss of the HNLF was increased by 3 to $4 \%$ when it is cooled to $77 \mathrm{~K}$.

Then, we obtain the values of $\boldsymbol{S}_{\boldsymbol{I}}$ and $\boldsymbol{S}_{2}$ from the curve fittings. When the HNLF was at room temperature the $\boldsymbol{S}_{\boldsymbol{I}}=0.000013$ and $\boldsymbol{S}_{2}=0.000034$ for Signal and $\boldsymbol{S}_{\boldsymbol{I}}=0.000011$ and $\boldsymbol{S}_{2}=0.000030$ for Idler. On average, the ratio of $\boldsymbol{S}_{2}$ to $\boldsymbol{S}_{\boldsymbol{I}}$ was about 2.7 when the HNLF was at room temperature ( $300 \mathrm{~K})$. When the HNLF was cooled to $77 \mathrm{~K}, \boldsymbol{S}_{\boldsymbol{I}}=0.0000012$ and $\boldsymbol{S}_{2}=0.000011$ for signal and $\boldsymbol{S}_{\boldsymbol{I}}=0.0000012$ and $\boldsymbol{S}_{2}=$ 
0.000012 for idler. It was observed that the ratio of $\boldsymbol{S}_{2}$ to $\boldsymbol{S}_{1}$ improved to about 9.6 when the HNLF was cooled to $77 \mathrm{~K}$, which means that most of the detection events resulted from the correlated photons generated from SFWM. The improvement of $\boldsymbol{S}_{2}$ to $S_{1}$ ratio is attributed to the suppression of Raman scattering at $77 \mathrm{~K}$ as discussed in section 4.4.

\subsubsection{Characterization of Correlated Photon Source}

\section{Coincidence Count and Accidental-coincidence Count}

Measurement of total coincidence count and accidental-coincidence count through the histogram plot in Fig. 4.5 is carried out at different pump photon per pulse. The results of coincident count and accidental coincidence count as a function of number of pump photon per pulse with HNLF at $300 \mathrm{~K}$ and $77 \mathrm{~K}$ are shown in Fig. 4.12 and Fig. 4.13, respectively. Total coincidence count and accidental-coincidence count increased with increasing number of pump photon per pulse. It was attributed to fact that the generation of both correlated photon-pairs by SFWM and noise photons by Raman scattering increased with the number of total pump photons. The net coincidence count that represents the detections of correlated photon-pairs generated by SFWM was obtained by subtracting the accidental coincidence count from total coincidence count. 


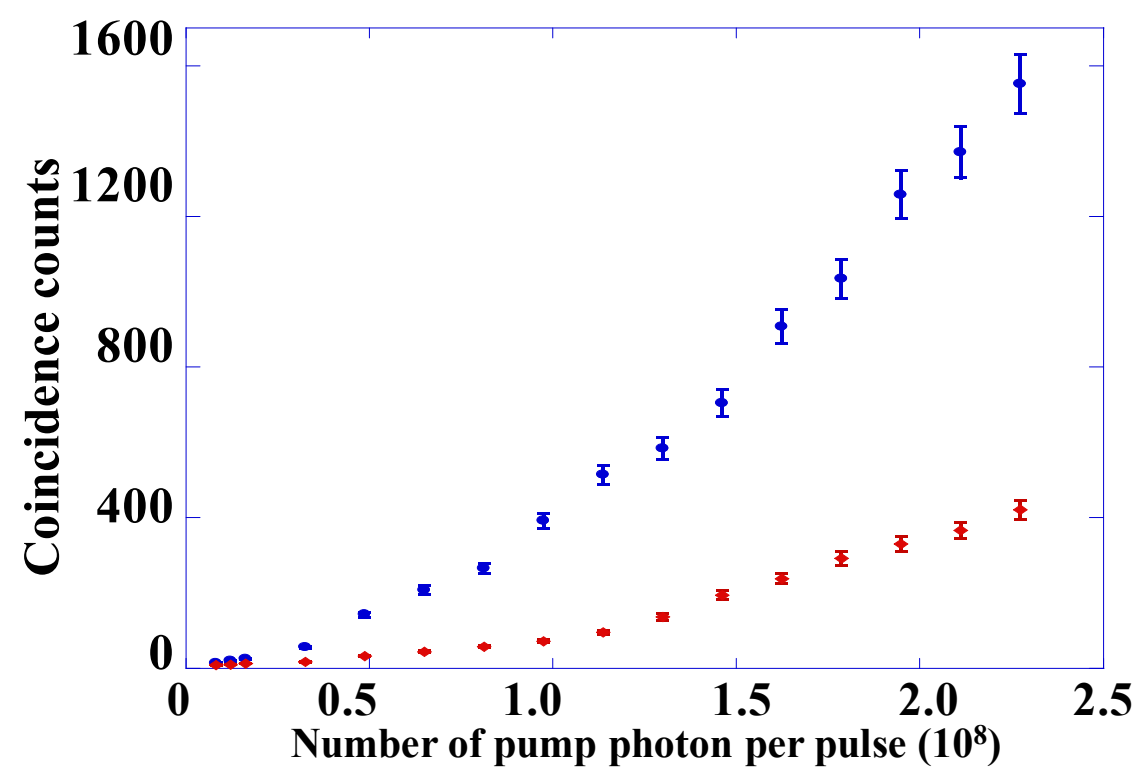

Figure 4.12. A plot of total coincidence counts (Blue dot) and accidental coincidence counts (Red diamond) as a function of number of pump photon per pulse with the HNLF in room temperature $(300 \mathrm{~K})$.

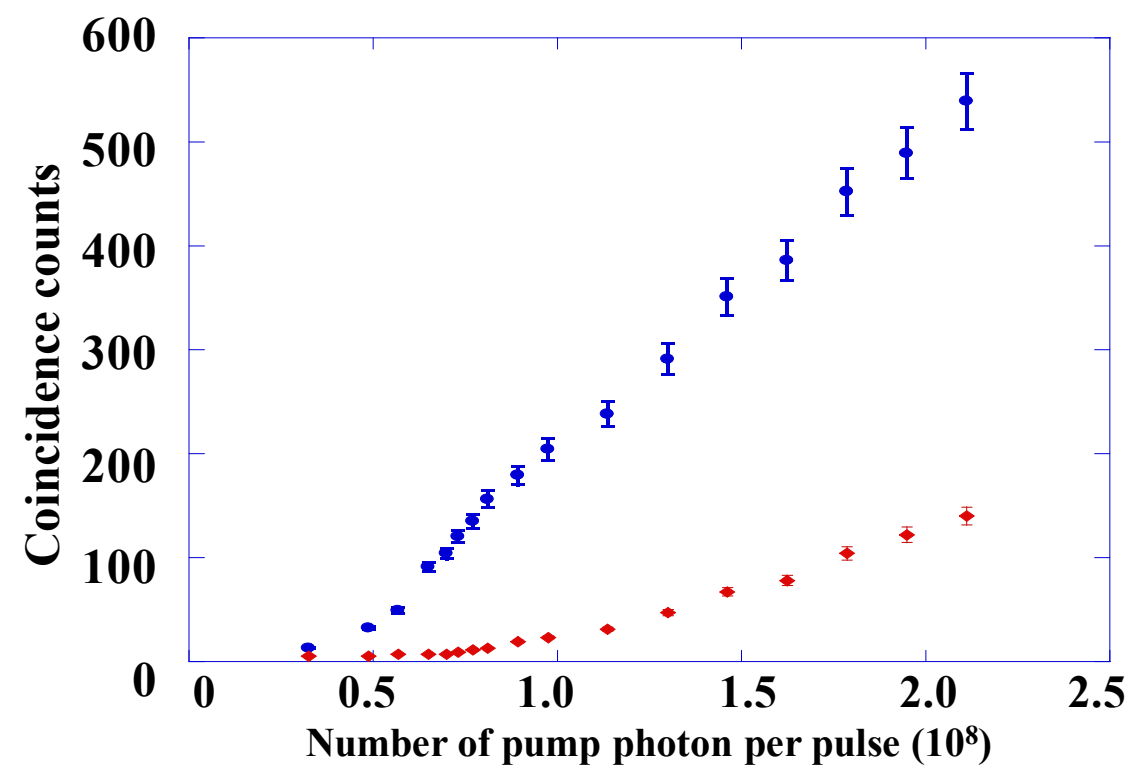

Figure 4.13. A plot of total coincidence counts (Blue dot) and accidental coincidence counts (Red diamond) as a function of number of pump photon per pulse with the HNLF in $77 \mathrm{~K}$. 
Comparing the plots for the HNLF in $300 \mathrm{~K}$ and $77 \mathrm{~K}$, total coincidence count and accidental-coincidence count decreased when the HNLF was cooled to 77 K. Decrease in total coincidence count and accidental-coincidence count were mainly due to the suppression of Raman photon and additional propagation loss of the HNLF when it was cooled to $77 \mathrm{~K}$. Nonlinear dependence of accidental coincident counts was observed at high pump photon number regime. This is contradicting with Raman scattering, which is linearly dependent on pump power. At high pump photon number, multiple photon pairs were generated and caused the nonlinear increase in accidental coincident count.

The quality of the correlated photon source is determined by the purity of the correlated photon-pairs. It is important to determine the optimum ratio of the correlated photon-pairs (by SFWM) to the noise photons (Raman scattering). Hence, we use the data from Fig. 4.12 and Fig. 4.13 to plot out the coincidence count to accidental coincidence count ratio in next section.

\section{Coincidence to Accidental-coincidence Ratio}

Coincidence to accidental-coincidence ratio (CAR) plot was used as the figure of merit to determine purity of a photon-pair generated in a HNLF. Fig.4.14 shows the measurement of CAR for different number of pump photon per pulse at $300 \mathrm{~K}$ and $77 \mathrm{~K}$. High CAR value means most of the recorded coincidence events arised from correlated photon-pairs by SFWM and Raman noise photons which induce accidental-coincidence are relatively limited. 


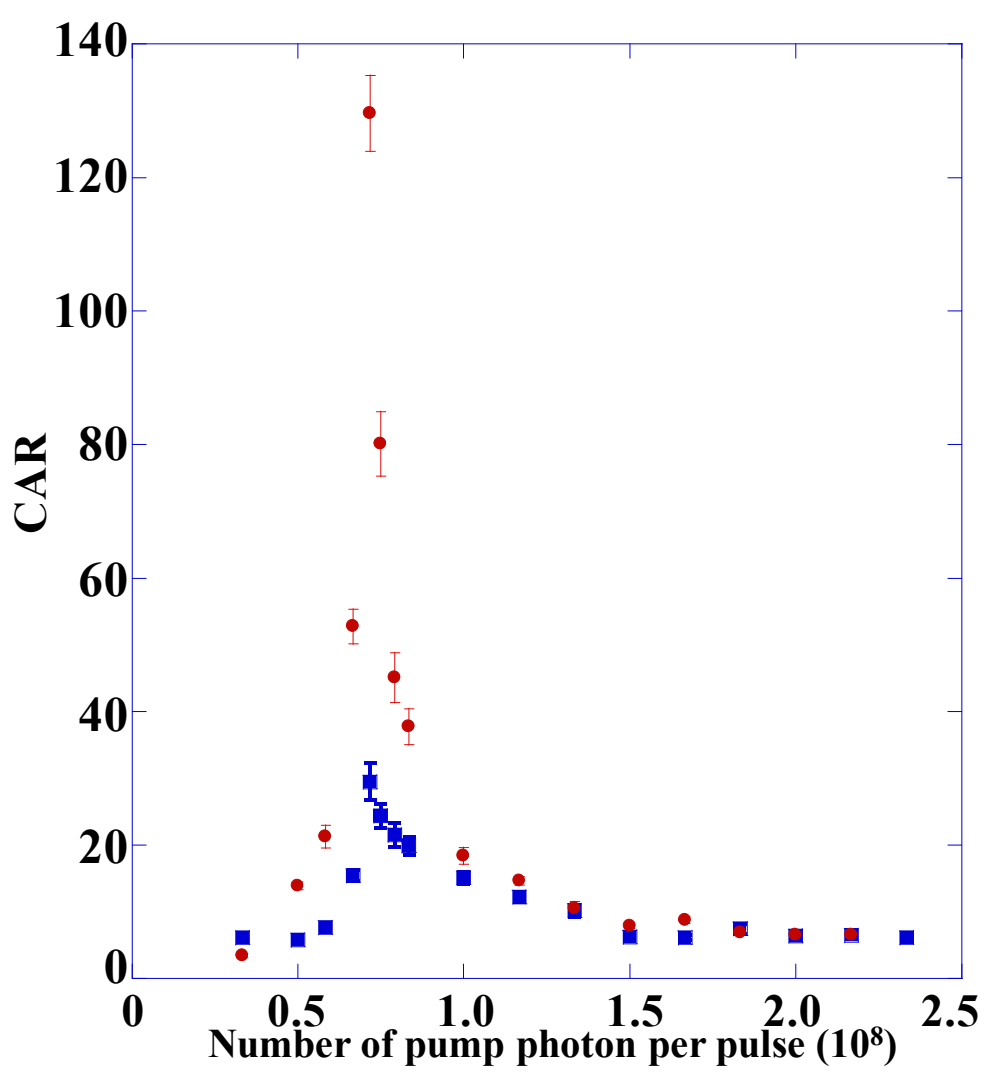

Figure 4.14. Coincidence to accidental coincidence ratio (CAR) versus number of pump photon per pulse with HNLF at $300 \mathrm{~K}$ (Blue dot) and $77 \mathrm{~K}$ (Red triangle).

Cooling the HNLF to $77 \mathrm{~K}$ significantly improved the CAR, indicating higher purity of correlated photon-pair was generated with lower temperature. Optimum CAR values of about 29 and 130 were obtained at $300 \mathrm{~K}$ and $77 \mathrm{~K}$ respectively, both with number of pump photons per pulse at about $7 \times 10^{7}$. This optimum phase condition was achieved with the pump, signal, and idler wavelengths that used in our experiment. The CAR value of 130 at $77 \mathrm{~K}$ was significantly higher than the CAR value of 29 at $300 \mathrm{~K}$.

Using CAR plot, we determined the optimum condition for the generation of high purity correlated photon pair with $10 \mathrm{~m}$ long HNLF. The optimization studies of correlated 
photon- pair will provide the cornerstone for polarization-entanglement experiments in next two sections.

\subsubsection{Characterization of Entangled Photon Source}

\section{Two-photon Interference}

For two photon interference (TPI) experiment, we created the polarization-entangled two photon state

$$
\left|\Psi^{+}\right\rangle=\frac{1}{\sqrt{2}}\left[\left|H_{i} H_{s}\right\rangle+\left|V_{i} V_{s}\right\rangle\right]
$$

as described in experimental setup section. The optimum number of pump photons per pulse was used as determined in last section for both horizontally and vertically polarized pump pulses. In this experiment, we set $\theta_{1}=0^{\circ}$ (and later $\theta_{1}=-45^{\circ}$ ) in signal channel and varied $\theta_{2}$ in idler channel, and recorded the coincidence counts for integration time of $68 \mathrm{~s}$. The results of two-photon interference (TPI) measurements as a function of relative angle $\theta_{2}-\theta_{1}$ with HNLF at $300 \mathrm{~K}$ and $77 \mathrm{~K}$ are shown in Fig. 4.15 and Fig. 4.16 respectively.

The fidelity of the two-photon polarization-entangled state was examined by measuring the visibility of two-photon interference fringe of signal and idler photons. We defined the visibility as,

$$
\mathcal{V}_{e n t}=\frac{\mathrm{CC}_{(\max )}-\mathrm{CC}_{(\min )}}{\mathrm{CC}_{(\max )}+\mathrm{CC}_{(\min )}}
$$

where $\mathrm{CC}_{(\max )}$ is the maximum coincidence and $\mathrm{CC}_{(\max )}$ is the minimum coincidence. When the HNLF was at $300 \mathrm{~K}$, two-photon interference fringe with the visibility $>92 \%$ was observed. The visibility of TPI $>98 \%$ was obtained when HNLF was cooled to $77 \mathrm{~K}$. 
All these measurements were obtained without subtracting the accidental coincidence counts due to Raman or any background noise photons. Only detector dark counts were subtracted from the coincidence counts. The coincidence detection rate was mainly limited by the slow detection rate of the single photon detectors and coincidence detection system that was used in our experiment.

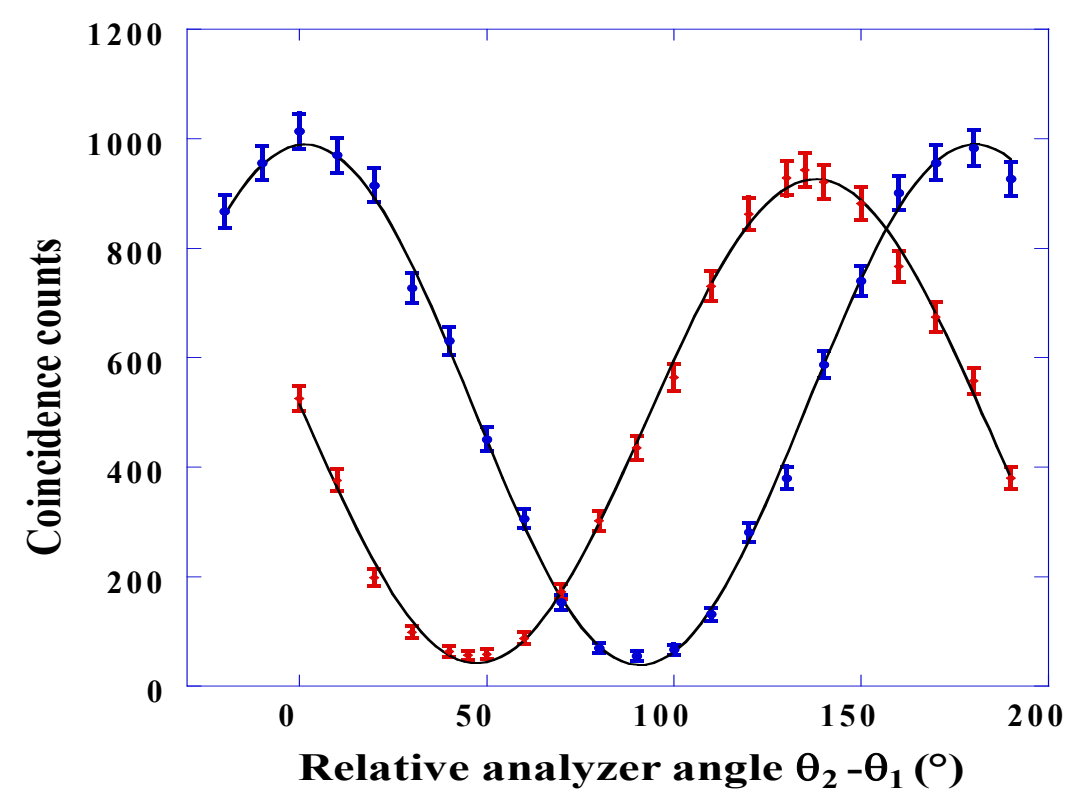

Figure 4.15. Two-photon interference fringes with HNLF at $300 \mathrm{~K}$ with (i) $\theta_{1}=0^{\circ}$ (Blue dot) and (ii) $\theta_{1}=-45^{\circ}$ (Red dot). The solid lines are the theoretical curve fitting. 


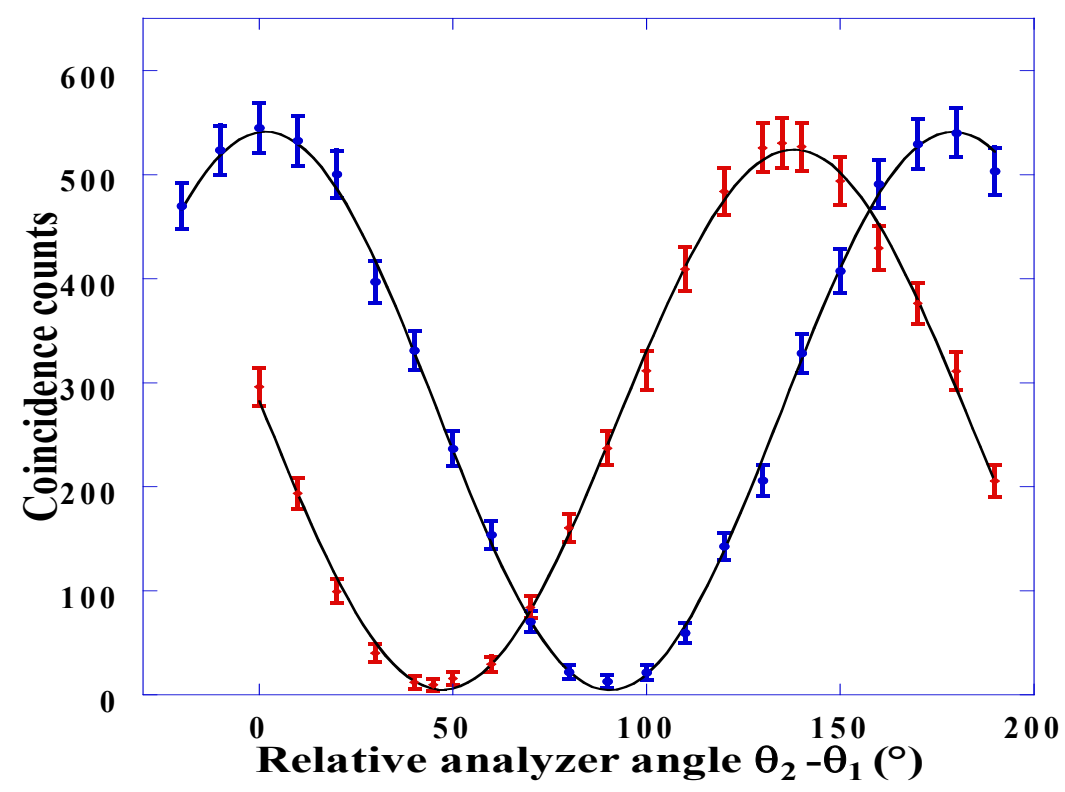

Figure 4.16. Two-photon interference fringes with HNLF at $77 \mathrm{~K}$ (i) $\theta_{1}=0^{\circ}$ (Blue dot) and (ii) $\theta_{1}=-45^{\circ}$ (Red dot). The solid lines are the theoretical curve fitting

\section{Violation of CSHS Bell's inequality}

Bell's inequality violation test was performed to verify the non-locality behavior of the polarization-entangled photon pair generated in the HNLF. In our experiment, we prepared the polarization-entangled state $\frac{1}{\sqrt{2}}\left[\left|H_{i} V_{S}\right\rangle-\left|V_{i} H_{S}\right\rangle\right]$ by inserting a QWP at $0^{\circ}$ after HWP1. An additional of a HWP at $45^{\circ}$ in signal channel rotates the polarization of signal photon from horizontal (vertical) to vertical (horizontal). Then, we measured the $|\mathrm{S}|$ parameter using Clauser, Horne, Shimony, and Holt (CSHS) form of Bell's inequality, $|\mathrm{S}| \leq$ 2 [55]. CSHS form of Bell's inequality provides the upper bound of $|\mathrm{S}|$ parameter measurement of local physical system as 2 . A violation $(|S|>2)$ of this inequality implies that polarization-entangled photon pair generated in the HNLF does not behave locally. 
Coincidence counts of 16 different combination analyzer settings with $\theta_{l}=0^{\circ}, \theta_{l}^{\prime}=-$ $45^{\circ}, \theta_{1}^{\perp}=90^{\circ}, \theta_{1}^{\prime \perp}=45^{\circ}$, and $\theta_{2}=67.5^{\circ}, \theta_{2}^{\prime}=22.5^{\circ}, \theta_{2}^{\perp}=-22.5, \theta_{2}^{\prime \perp}=112.5$ were recorded for polarization-entangled state $\left|H_{i} V_{s}\right\rangle-\left|V_{i} H_{s}\right\rangle$ with $\mathrm{HNLF}$ at both $300 \mathrm{~K}$ and $77 \mathrm{~K}$. The measurement of Bell's inequality violation with HNLF at $300 \mathrm{~K}$ and $77 \mathrm{~K}$ are shown in Table 4.1. At $300 \mathrm{~K}$ we obtained $|\mathrm{S}|=2.267 \pm 0.054$, which violates Bell's inequality by close to 5 standard deviations. When the HNLF was cooled to $77 \mathrm{~K},|\mathrm{~S}|=2.788 \pm 0.064$, the violation of Bell's inequality by more than 12 standard deviations was observed. Larger violation of Bell's inequality was observed at $77 \mathrm{~K}$ as the coincidences were mostly contributed from the non-locally correlated photon pair and not other noise photon. The standard deviation was derived from the Poisson statistic error of the single photon detection. Violation of Bell's inequality attests that the polarization-entangled two-photon states generated in our experiment cannot be described by local hidden variable theory.

Table 4.1 Violation of Bell's inequality for entangled state $\left|\boldsymbol{H}_{i} \boldsymbol{V}_{\boldsymbol{s}}\right\rangle-\left|\boldsymbol{V}_{\boldsymbol{i}} \boldsymbol{H}_{\boldsymbol{s}}\right\rangle$

\begin{tabular}{ccc}
\hline \hline Temperature $(\mathrm{K})$ & $|\mathrm{S}|$ & Violation \\
\hline 300 & $2.267 \pm 0.054$ & $4.95 \sigma$ \\
\hline 77 & $2.788 \pm 0.064$ & $12.31 \sigma$ \\
\hline
\end{tabular}

\subsection{Discussions}

We demonstrated the generation of correlated and polarization-entangled photon pair at telecom wavelength via spontaneous four-waves mixing using a $10 \mathrm{~m}$ long highly nonlinear fiber in a counter propagating scheme. 
We observed coincidence to accidental coincidence ratio (CAR) of $29 \pm 3$ at room temperature $(300 \mathrm{~K})$ and as high as $130 \pm 5$ when the fiber is cooled to liquidnitrogen temperature $(77 \mathrm{~K})$. The improvement factor obtained in experiment is comparable to the theoretical value that we calculated based on the temperature dependence of Raman photon in section 4.4. The photon-pair production rates at these optimum CAR values are about $0.03(300 \mathrm{~K})$ and $0.02(77 \mathrm{~K})$ per pulse. They were higher than production rate obtained in $300 \mathrm{~m}$ long dispersion shifted fiber [120]. The trend observed for CAR measurements at both temperatures are similar to those obtained by different type of entangled photons sources [ $[\underline{98}, \underline{121}, \underline{135}]$. Low CAR values at low pump photon number were due to non optimum phase matching condition. At high pump photon number, multi-photon pairs effect that aroused from generation of more than a photon-pair increased the accidental-coincidence count thus led to low CAR values.

For the two photon interference experiment, we obtained two-photon interference fringe with the visibility $>92 \%$ in HNLF at $300 \mathrm{~K}$, while the visibility of TPI $>98 \%$ was observed when HNLF was cooled to $77 \mathrm{~K}$. The lower visibility at $300 \mathrm{~K}$ is likely due to the contamination of Raman photons, which give rise to accidental coincidence counts. On the other hand, higher visibility at $77 \mathrm{~K}$ was primarily limited by the poor detection efficiencies of the detectors and the remaining background noise photons. The normalized coincidence count that we obtained in the two-photon interference experiment is equivalent to expectation value of two-photon detection for polarization-entangled states $\left|\Psi^{+}\right\rangle$. 
Consider that the $\mathrm{x}$-direction as horizontal component and $\mathrm{y}$-direction as vertical component, the polarization-entangled two photon state can be written as,

$$
\left|\Psi^{+}\right\rangle=\frac{1}{\sqrt{2}}\left[\left|1_{s, x}, 0_{s, y}, 1_{i, x}, 0_{i, y}\right\rangle+\left|0_{s, x}, 1_{s, y}, 0_{i, x}, 1_{i, y}\right\rangle\right]
$$

where $\left|1_{s, x}\right\rangle$ denotes the state in which the signal photon is linearly polarized in $\mathrm{x}$-direction (horizontal) and $\left|1_{i, y}\right\rangle$ denotes the state in which the idler photon is linearly polarized in $y$ direction (vertical), etc. Given that the projection angle of polarization analyzers for signal and idler photon are $\theta_{1}$ and $\theta_{2}$ with respect to x-direction. After the polarization analyzers, the annihilation operators in $\mathrm{x}$ and $\mathrm{y}$ directions for signal and idler photon are given as [136],

$$
\begin{aligned}
& \hat{\mathrm{a}}_{1}=\hat{\mathrm{a}}_{s, x} \cos \theta_{1} \hat{x}+\hat{\mathrm{a}}_{s, y} \sin \theta_{1} \hat{y}, \\
& \hat{\mathrm{a}}_{2}=\hat{\mathrm{a}}_{i, x} \cos \theta_{2} \hat{x}+\hat{\mathrm{a}}_{i, y} \sin \theta_{2} \hat{y} .
\end{aligned}
$$

From Eqs. 4.20, 4.21 and 4.22, the probability signal and idler photon to be detected by of APD1 and APD2 are,

$$
\begin{aligned}
& \mathcal{P}_{1}\left(\theta_{1}\right)=\alpha_{1}\left\langle\Psi^{+}\left|\hat{\mathrm{a}}_{1}^{\dagger} \hat{\mathrm{a}}_{1}\right| \Psi^{+}\right\rangle=\frac{1}{2} \alpha_{1}, \\
& \mathcal{P}_{2}\left(\theta_{2}\right)=\alpha_{2}\left\langle\Psi^{+}\left|\hat{\mathrm{a}}_{2}^{\dagger} \hat{\mathrm{a}}_{2}\right| \Psi^{+}\right\rangle=\frac{1}{2} \alpha_{2} .
\end{aligned}
$$

Where, $\alpha_{1}$ and $\alpha_{2}$ are quantum efficiencies of APD1 and APD2. The joint probability that both signal and idler photon are detected by APD1 and APD2 with the projection angle of polarization analyzers at $\theta_{1}$ and $\theta_{2}$ are,

$$
\begin{aligned}
\mathcal{P}_{12}\left(\theta_{1}, \theta_{2}\right) & =\alpha_{1} \alpha_{2}\left\langle\Psi^{+}\left|\hat{\mathrm{a}}_{1}^{\dagger} \hat{\mathrm{a}}_{2}^{\dagger} \hat{\mathrm{a}}_{2} \hat{\mathrm{a}}_{1}\right| \Psi^{+}\right\rangle \\
& =\frac{1}{2} \alpha_{1} \alpha_{2} \cos ^{2}\left(\theta_{2}-\theta_{1}\right) \\
& \propto \cos ^{2}\left(\theta_{2}-\theta_{1}\right) .
\end{aligned}
$$


Then, the joint probability of two-photon detection for polarization-entangled is dependent on the relative projection angle of two polarization analyzers at signal and idler channel. It can be predicted that maximum probability of the two-photon detection can be obtained at $\left\{\left(\theta_{2}-\theta_{1}\right)=0\right.$ and $\left\{\left(\theta_{2}-\theta_{1}\right)=180\right\}$. Minimum probability of two-photon detection can be obtained at $\left\{\left(\theta_{2}-\theta_{1}\right)=90\right\}$. The two photon interference fringes obtained in the Fig. 4.15 and Fig. 4.16 matches well with the theoretical prediction.

We verified the non-locality of the polarization entangled photon pairs by observing the violation of Bell's inequality by $>12$ standard deviations at $77 \mathrm{~K}$ and $\approx 5$ standard deviations at $300 \mathrm{~K}$, respectively. The $|\mathrm{S}|$ parameter measured in our experiment is given by

$$
|S|=\left|E\left(\theta_{1}, \theta_{2}\right)-E\left(\theta_{1}, \theta_{2}^{\prime}\right)\right|+\left|E\left(\theta_{1}^{\prime}, \theta_{2}\right)+E\left(\theta_{1}^{\prime}, \theta_{2}^{\prime}\right)\right| \leq 2,
$$

$E\left(\theta_{1}, \theta_{2}\right)$ is expressed as

$$
E\left(\theta_{1}, \theta_{2}\right)=\frac{R\left(\theta_{1}, \theta_{2}\right)+R\left(\theta_{1}^{\perp}, \theta_{2}^{\perp}\right)-R\left(\theta_{1}, \theta_{2}^{\perp}\right)-R\left(\theta_{1}^{\perp}, \theta_{2}\right)}{R\left(\theta_{1}, \theta_{2}\right)+R\left(\theta_{1}^{\perp}, \theta_{2}^{\perp}\right)+R\left(\theta_{1}, \theta_{2}^{\perp}\right)+R\left(\theta_{1}^{\perp}, \theta_{2}\right)}
$$

where $\theta_{1,2}^{\perp}=\theta_{1,2}+90^{\circ}$ and $R\left(\theta_{1}, \theta_{2}\right)$ is the coincidence count that we measure in experiment when the analyzers projection angle set to $\theta_{1}, \theta_{2}$. 



\section{Chapter 5}

\section{Photon-pairs Propagate Through Loss and Scattering Media ${ }^{4}$}

\subsection{Overview}

In this chapter, we investigate the quantum correlation and interference of fiber based photon-pair at telecom wavelengths with one photon experiencing standard loss or multiple scattering in a random medium.

We will begin with a short introduction on motivation of our work in this chapter. Then we propose a model on the evolution of annihilation operator with one photon of the photon pair goes through multiple scattering in random medium, where the annihilation operators for signal photon propagates in normal channel $\hat{a}_{1}$ and idler photon scattered through random media $\left(\hat{\mathrm{a}}_{2^{\prime}}\right)$ are described in detail. Also, we going to introduce the operators associated with the coherent $\left(\hat{\mathrm{a}}_{\mathrm{o}}\right)$ and incoherent $(\widehat{\mathrm{c}})$ part of the scattering process in random medium.

\footnotetext{
${ }^{4}$ The material contained in this chapter has been submitted to Optics Letters.
} 
In the following section, the joint probability of two-photon detection $\mathcal{P}_{12}=$ $\left\langle\psi\left|\hat{a}_{1}^{\dagger} \hat{a}_{2}^{\dagger}, \hat{a}_{2}, \hat{a}_{1}\right| \psi\right\rangle$ for both polarized polarization-correlated/-entangled photon pair will be explained in detail. We will disclose how the visibility of polarizationentangled photon pair $\left(\mathcal{V}_{\text {ent }}\right)$ and predicted visibility of polarization-correlated photon pair $\left(\mathcal{V}_{\text {cor }}\right)$ are associated with the transmission amplitude $\mathcal{T}$ and depolarization amplitude $\mathcal{L}$ of the scattered photon in a random medium. $\left(\mathcal{V}_{\text {cor }}\right)$ is the predicted visibility if the polarization-correlated photon pair is used to generate polarization-entangled state.

Then, we describe the preparation of multiple scattering random media that were used in our experiment. The multiple scattering samples in a quartz cuvette are prepared by dispersing uniform polystyrene microspheres in oil suspension. In our experiment, oil suspension medium is used due to the high absorption of water in photon-pair's wavelengths around $1.55 \mu \mathrm{m}$. Details of samples preparation by mixing the polystyrene microspheres in oil are described and physical properties of the all random media are tabulated in this section.

Experiment setup for generation and detection of polarization-correlated/entangled photon-pair is the same as described in section 4.6. Therefore, in this section we focus on the description of experimental setup in sending one photon of the photon pair through a normal channel and the other photon experiencing standard loss or multiple scattering in a random medium. We measure joint probability of twophoton detection that discussed in Section 5.4 by the means of coincidence detection. 
In the coming section of experiment results, we first present results on the effect of standard loss on quantum correlation and interference of photon-pair. We obtain $\mathcal{V}_{\text {ent }}$ and $\mathcal{V}_{\text {cor }}$ as a function of standard losses $(1 \mathrm{~dB}, 3 \mathrm{~dB}$ and $5 \mathrm{~dB})$. We found that $\mathcal{V}_{\text {ent }}$ and $\mathcal{V}_{\text {cor }}$ are decreasing in line with attenuation proves that standard loss in transmission channel. It is also observed that both polarization correlated and entangled photon pair are equally sensitive to the standard losses. For investigation on the scattering media, $\mathcal{V}_{\text {ent }}$ and $\mathcal{V}_{\text {cor }}$ as a function of mean free-path $\left(\ell_{1,2,3,4}\right)$ of $0.019 \mathrm{~m}, 0.010 \mathrm{~m}, 0.004 \mathrm{~m}$, and $0.003 \mathrm{~m}$ are presented. We show that $\mathcal{V}_{\text {ent }}$ and $\mathcal{V}_{\text {cor }}$ are decreasing with shorter scattering mean free path of the random media. In addition, our results prove that quantum correlation of polarization entangled photon pair is better preserved than polarization-correlated photon-pair as one photon of the photon-pair experiences random scattering process in the random medium. Then, we study the role of Raman photon in depolarization of the idler photon in scattering process, where our results evident that Raman noise photon in photon-pair source before entering a random medium will enhance the depolarization effect.

\subsection{Introduction}

Since its introduction by Bennett and Brassad in 1984 [12], quantum key distribution has attracted great interest among researchers. The prospect of secured communication based on laws of quantum mechanics spurs on its eccentric progress over the years. Correlated or entangled photon pair have been proposed and 
extensively investigated as the information carriers for quantum key distribution [137, $\underline{138] .}$

Despite the success of demonstrating entanglement based quantum key distribution over $100 \mathrm{~km}$ of fiber and $144 \mathrm{~km}$ of free space channel the practicality of using correlated or entangled photon pair for long distance or eventually global quantum key distribution $(\mathrm{QKD})$ remained in doubt $[\underline{30}, \underline{42}]$. For free space channel, question on the feasibility of global scale QKD arises due to major limitations such as atmospheric scattering, turbulence and propagation losses $[\underline{32}, \underline{33}]$. On the other hand, linear propagation and connection loss with current optical fiber technology are limiting the fiber network distance for quantum key distribution. In short, it requires the quantum correlations to be preserved over the disturbances in transmission channel when separating the photon pair over large scale.

Hence, explicit investigation on the propagation of the correlated or entangled photon pair through scattering medium and linear losses is a great interest from the perspective of fundamental quantum physics. It is also equally pivotal for the implementation of various entanglement based QKD. Various computational and theoretical studies have been carried out in order to provide insight on the effects of scattering medium and linear losses on the quantum correlation of photons $[139,140]$. Recent theoretical treatments of quantum light and entanglement in the turbulent atmosphere did not include the depolarization effect of the scattered photon [141]. It is the goal of this work to describe an experimental study on the effect of scattering medium and standard loss on quantum correlation of correlated or entangled photon 
pair. We experimentally investigated the propagation of polarization-correlated and polarization-entangled photon pair at telecom wavelength through a non-absorbing, multiple scattering random medium. In addition, we also measured the deterioration of quantum correlation of photon pair due to the linear optical attenuation in the transmission channel. In particular, our experimental setup was modeled in analogues to the entanglement based QKD demonstrated by other research groups, where one photon from the entangled pair was measured locally and second photon was sent via a transmission channel with loss or disturbances [30, $\underline{43}]$. Fiber based correlated and entangled photon sources at telecom wavelength was chosen due to its well defined spatial mode and excellent transmittance in both atmospheric channel and optical fiber for long distance distribution, storage and processing [142].

\subsection{Evolution of Annihilation Operator}

In the proposed model with one photon (idler) of the photon pair passed through the multiple scattering in random medium. The signal photon of the photon pair was propagating in the normal channel with negligible loss.

When the idler photon scatters through a random medium, annihilation operator $\hat{a}_{i}$ of the idler photon will evolve into coherent and incoherent parts. The coherent part is associated with the operator $\hat{a}_{o}$ and incoherent part is associated with operator $\hat{\mathrm{c}}$ as shown in Fig 5.1. 


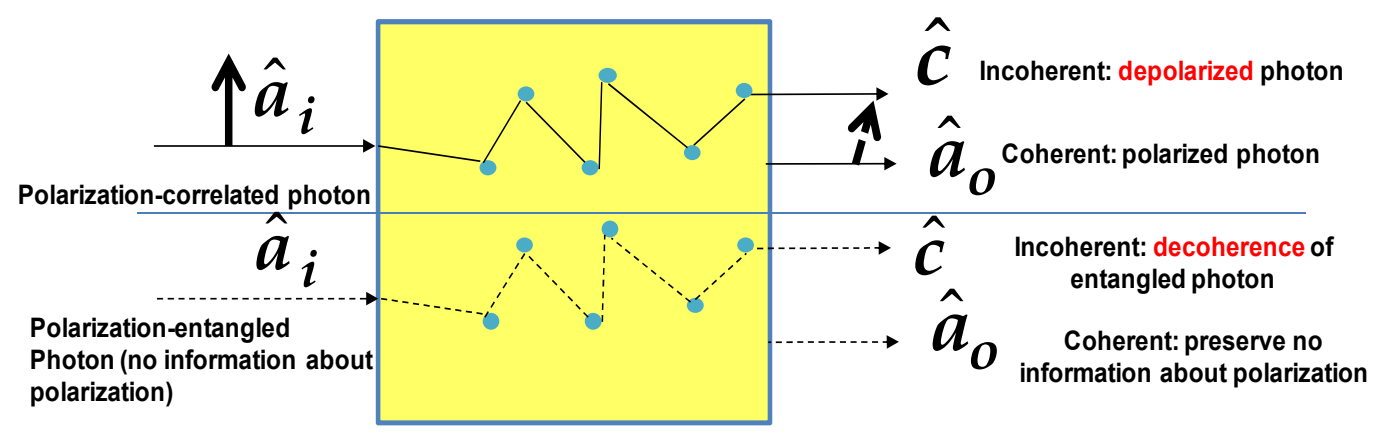

Figure 5.1. A simple model for one photon of polarization-correlated/-entangled photon-pair propagating through a random medium, where annihilation operator $\hat{a}_{i}$ of the idler photon goes through coherent $\left(\hat{a}_{0}\right)$ and incoherent $(\hat{c})$ part of the scattering process in random medium.

The operator $\hat{a}_{\mathrm{o}}$ corresponds to the ballistic photon that undergoes no depolarization. For a polarization-correlated photon, $\hat{a}_{\mathbf{o}}$ is operator for the photon that will preserve the input of horizontally (vertically) polarized light. The polarization state change in the output light can be compensated by using a combination of a quarter-wave plate and a half-wave plate. For a polarizationentangled photon, $\hat{a}_{\mathrm{o}}$ is for the photon that will preserve no information of polarization state of the input photon. While $\hat{\mathrm{c}}$ is noise annihilation operator corresponding to photon that undergoes depolarization. This operator accounts for incoherent part of the incoming photon through multiple scattering events in the random medium. Therefore, the operator $\hat{\mathrm{c}}$ is for the photon that will undergo depolarization in the case of polarization-correlated photon; and for the photon that will experience decoherence in the case of polarization-entangled photon. For coherent component, the operator $\hat{a}_{o}$ is given as 


$$
\hat{\mathrm{a}}_{\mathrm{o}}=\mathcal{T} \hat{\mathrm{a}}_{i}
$$

where $\mathcal{T}$ is the transmission amplitude of the idler photon.

The depolarization amplitude associated for operator $\hat{\mathrm{c}}$ is defined as $\mathcal{L}=\frac{\mathcal{A}}{\ell}$, where $\ell$ is scattering mean free-path of the random medium and $\mathcal{A}$ is depolarization constant in the unit of meter. The depolarization constant $\mathcal{A}$ can be inferred from our experimental data which consists of depolarization photons and Raman noise photons.

After pass through random medium, the output annihilation operator can be decomposed into orthogonal $x-y$ polarization axis, given by

$$
\hat{\mathrm{a}}_{2 \mathrm{o}^{\prime}}=\hat{\mathrm{a}}_{2 x^{\prime}} \hat{x}+\hat{\mathrm{a}}_{2 y^{\prime}} \hat{y}
$$

Where "2" corresponds to the idler photon passed through channel 2. The annihilation operator in $\mathrm{x}$ and $\mathrm{y}$ axis including coherent and incoherent component are given as

$$
\begin{aligned}
& \hat{\mathrm{a}}_{2 x^{\prime}}=\mathcal{T}_{x} \hat{\mathrm{a}}_{i, x}+\mathcal{L}_{x} \hat{\mathrm{c}}, \\
& \hat{\mathrm{a}}_{2 y^{\prime}}=\mathcal{J}_{y} \hat{\mathrm{a}}_{i, y}+\mathcal{L}_{y} \hat{\mathrm{c}} .
\end{aligned}
$$

The operator $\hat{c}$ is annihilation operator for depolarization which can be operated independent of polarization axis. For a homogenous random medium, the depolarization amplitude in the $\mathrm{x}-\mathrm{y}$ polarization axis are equal and given as 


$$
\mathcal{L}_{x}=\mathcal{L}_{y}=\frac{1}{\sqrt{2}} \mathcal{L}
$$

Similarly the transmission amplitude in the $\mathrm{x}-\mathrm{y}$ polarization axis are given by,

$$
\mathcal{T}_{x}=\mathcal{T}_{y}=\frac{1}{\sqrt{2}} \mathcal{T}
$$

Then the output annihilation operator in Eq. 5.2 can be written accordingly

$$
\begin{aligned}
\hat{\mathrm{a}}_{2 \mathrm{o}^{\prime}} & =\left(\mathcal{T}_{x} \hat{\mathrm{a}}_{i, x}+\mathcal{L}_{x} \hat{\mathrm{c}}\right) \hat{x}+\left(\mathcal{T}_{y} \hat{\mathrm{a}}_{i, y}+\mathcal{L}_{y} \hat{\mathrm{c}}\right) \hat{y} \\
& =\frac{1}{\sqrt{2}}\left[\left(\mathcal{T} \hat{\mathrm{a}}_{i, x}+\mathcal{L} \hat{\mathrm{c}}\right) \hat{x}+\left(\mathcal{J} \hat{\mathrm{a}}_{i, y}+\mathcal{L} \hat{\mathrm{c}}\right) \hat{y}\right] .
\end{aligned}
$$

Now, suppose a polarizer is placed after the random medium and inclined at projection angle $\theta_{2}$ to project the scattered photon to the polarization state

$$
\hat{e}=\cos \theta_{2} \hat{x}+\sin \theta_{2} \hat{y}
$$

The photon annihilation operator after the polarizer becomes,

$$
\begin{gathered}
\hat{\mathrm{a}}_{2 \prime}=\hat{\mathrm{a}}_{2 \mathrm{o}^{\prime}} \cdot \hat{e}=\mathcal{T}_{x} \hat{\mathrm{a}}_{i, x} \cos \theta_{2}+\mathcal{T}_{y} \hat{\mathrm{a}}_{i, y} \sin \theta_{2}+\left(\mathcal{L}_{x}+\mathcal{L}_{y}\right) \hat{\mathrm{c}} \\
=\frac{1}{\sqrt{2}}\left[\mathcal{T}\left(\hat{\mathrm{a}}_{i, x} \cos \theta_{2}+\hat{\mathrm{a}}_{i, y} \sin \theta_{2}\right)+(2 \mathcal{L}) \hat{\mathrm{c}}\right]
\end{gathered}
$$

The $\hat{\mathrm{c}}$ is not associated with $\cos \theta_{2}$ and $\sin \theta_{2}$ terms as the depolarization effect is independent of projection angle of the polarizer.

As for the signal photon which is propagating in the normal channel with the assumption of experiencing negligible loss, the photon annihilation operator of the signal photon after a polarizer with the projection angle $\theta_{l}$ is, 


$$
\hat{\mathrm{a}}_{1}=\hat{\mathrm{a}}_{s, x} \cos \theta_{1} \hat{x}+\hat{\mathrm{a}}_{s, y} \sin \theta_{1} \hat{y} .
$$

\subsection{Joint Probability of Two-photon Detection}

Suppose two single photon detectors are placed after the polarizer at both the normal channel and scattering random medium channel. The joint probability two-photon detection can be written as [136],

$$
\mathcal{P}_{12}=\left\langle\psi\left|\hat{\mathrm{a}}_{1}^{\dagger} \hat{\mathrm{a}}_{2}^{\dagger}, \hat{\mathrm{a}}_{2}, \hat{\mathrm{a}}_{1}\right| \psi\right\rangle
$$

where $\hat{\mathrm{a}}_{1}$ and $\hat{\mathrm{a}}_{2}$, are the photon annihilation operators for both channels and $\hat{\mathrm{a}}_{1}^{\dagger}$ along with $\hat{a}_{2 \text {, }}^{\dagger}$ are the photon creation operators.

\subsubsection{Polarization-correlated Photon Pair}

The two-photon state of horizontally polarized polarization-correlated photon pair is given by

$$
\left|\Psi_{\text {cor }}\right\rangle=\left|1_{s, x}, 0_{s, y}, 1_{i, x}, 0_{i, y}\right\rangle
$$

Where $\left|1_{s, x}\right\rangle$ denotes the state in which the signal photon is linearly polarized in $\mathrm{x}$ direction (horizontal) and $\left|\mathbf{1}_{i, y}\right\rangle$ denotes the state in which the idler photon is linearly polarized in y-direction (vertical), etc.

The joint probability of two-photon detection for $\left|\Psi_{\text {cor }}\right\rangle$ can be written as 


$$
\mathcal{P}_{\text {cor }}=\left\langle\Psi_{\text {cor }}\left|\hat{\mathrm{a}}_{1}^{\dagger} \hat{\mathrm{a}}_{2^{\prime}}^{\dagger} \hat{\mathrm{a}}_{2^{\prime}} \hat{\mathrm{a}}_{1}\right| \Psi_{\text {cor }}\right\rangle
$$

Suppose the polarizers in normal channel and scattering random medium channel are oriented at projection angle $\theta_{1}$ and $\theta_{2}$ with respect to horizontal axis. Then the joint probability $\mathrm{P}_{\text {cor }}$ can be obtained in term of $\theta_{1}$ and $\theta_{2}$ as,

$$
\mathcal{P}_{\text {cor }}=\frac{\mathcal{T}^{2}}{2} \cos ^{2} \theta_{2} \cos ^{2} \theta_{1}+2 \mathcal{L}^{2}+2 \mathcal{T} \mathcal{L} \cos \theta_{2} \cos \theta_{2}
$$

The terms involving $\mathcal{L}^{2}$ and $\mathcal{T} \mathcal{L}$ are corresponding to contribution from noise photons in joint probability of two-photon detection.

From experimental point of view, a coincidence count will be recorded when both APDs detected a photon at the same gated time interval. The accidental coincidence count is recorded when both APDs detected a photon at the adjacent gated time interval. By setting the polarizer projection angle $\theta_{1}=0^{\circ}$ and $\theta_{2}=0^{\circ}$, the Eq. 5.14 is reduced to

$$
\mathcal{P}_{\text {cor }}=\frac{\mathcal{T}^{2}}{2}+2 \mathcal{L}^{2}+2 \mathcal{T} \mathcal{L}
$$

The Eq. 5.15 corresponds to the coincidences that recorded by the detection system, which is contributed by a photon-pair and depolarized noise photon. The terms $2 \mathcal{L}^{2}$ and $2 \mathcal{T} \mathcal{L}$ are contributing to accidental coincidence which corresponds to depolarized noise photon. Hence the coincidence $(C C)$ to accidental $(A C)$ ratio $(C A R)$ in given as

$$
C A R=\frac{C C}{A C}=\frac{\mathcal{T}^{2}+4 \mathcal{L}^{2}+4 \mathcal{T} \mathcal{L}}{4 \mathcal{L}^{2}+4 \mathcal{T} \mathcal{L}}
$$


In addition to the depolarization photon, $\mathcal{L}$ contains the contribution from Raman noise photon in fiber based photon-pair source. The contribution of Raman noise photon in $\mathcal{L}$ was included in the CAR measurement. From the Eq. 5.16, we defined the visibility of the two-photon interference as

$$
\mathcal{V}_{c o r}=\frac{C C-A C}{C C+A C}=\frac{T^{2}}{\mathcal{T}^{2}+8 \mathcal{L}^{2}+8 \mathcal{H} \mathcal{L}} .
$$

The accidental coincidence sets the limit for the achievable minimum visibility of the two-photon interference when correlated photons were used to generate entangled state.

\subsubsection{Polarization-entangled Photon pair}

The two-photon state of polarization-entangled photon pair can be written as

$$
\left|\Psi_{\text {ent }}\right\rangle=\frac{1}{\sqrt{2}}\left[\left|1_{s, x}, 0_{s, y}, 1_{i, x}, 0_{i, y}\right\rangle+\left|0_{s, x}, 1_{s, y}, 0_{i, x}, 1_{i, y}\right\rangle\right],
$$

with the similar notation as described in previous section.

The joint probability two-photon detection for $\left|\Psi_{\text {ent }}\right\rangle$ is given as

$$
\mathcal{P}_{\text {ent }}=\left\langle\Psi_{\text {ent }}\left|\hat{a}_{1}^{\dagger} \hat{a}_{2^{\prime}}^{\dagger} \hat{\mathrm{a}}_{2^{\prime}} \hat{\mathrm{a}}_{1}\right| \Psi_{\text {ent }}\right\rangle .
$$

Similarly, $\mathcal{P}_{\text {ent }}$ can be written as a function of $\theta_{1}$ and $\theta_{2}$ as,

$$
\mathcal{P}_{\text {ent }}=\frac{1}{2}\left[\frac{\mathcal{T}^{2}}{2} \cos ^{2}\left(\theta_{2}-\theta_{1}\right)+8 \mathcal{L}^{2}+4 \mathcal{T} \mathcal{L} \cos \left(\theta_{2}-\theta_{1}\right)\right] .
$$


The $\mathcal{P}_{\text {ent }}$ in Eq.5.20 is two-photon interference as a function of relative polarizer projection angle $\left(\theta_{2}-\theta_{1}\right)$. $P_{\text {ent }}$ contains two maximum interference points and one minimum interference point. $\mathcal{P}_{\text {ent }}$ at the first maximum point at $\left\{\left(\theta_{2}-\theta_{1}\right)=\right.$ $\left.0^{\circ}\right\}$ and second maximum point at $\left\{\left(\theta_{2}-\theta_{1}\right)=180^{\circ}\right\}$ are given as

$$
\begin{gathered}
\mathcal{P}_{\text {ent }(\max 1)}=\mathcal{P}_{\text {ent }\left(\theta_{2}-\theta_{1}=0^{\circ}\right)}=\frac{1}{2}\left[\frac{\mathcal{J}^{2}}{2}+8 \mathcal{L}^{2}+4 \mathcal{T} \mathcal{L}\right], \\
\mathcal{P}_{\text {ent }(\max 2)}=\mathcal{P}_{\text {ent }\left(\theta_{2}-\theta_{1}=180^{\circ}\right)}=\frac{1}{2}\left[\frac{\mathcal{T}^{2}}{2}+8 \mathcal{L}^{2}-4 \mathcal{T} \mathcal{L}\right] .
\end{gathered}
$$

The average of these two maximum points is obtained as

$$
\begin{aligned}
\mathcal{P}_{\text {ent }(\max )} & =\frac{\mathcal{P}_{\text {ent }(\max 1)}+\mathcal{P}_{\text {ent }(\max 2)}}{2} \\
& =\frac{1}{2}\left[\frac{\mathcal{T}^{2}}{2}+8 \mathcal{L}^{2}\right]
\end{aligned}
$$

The only minimum interference point is obtained at $\left\{\left(\theta_{2}-\theta_{1}\right)=90^{\circ}\right\}$, with the joint probability given as

$$
\mathcal{P}_{\text {ent }(\min )}=\mathcal{P}_{\text {ent }\left(\theta_{2}-\theta_{1}=90^{\circ}\right)}=4 \mathcal{L}^{2}
$$

Consequently, we can calculate the visibility of the polarization-entangled twophoton state as given by,

$$
\begin{aligned}
\mathcal{V}_{\text {ent }} & =\frac{\mathcal{P}_{\text {ent }(\max )}-\mathcal{P}_{\text {ent }(\min )}}{\mathcal{P}_{\text {ent }(\max )}{ }^{\mathcal{P}_{\text {ent }(\min )}}} \\
& =\frac{\mathcal{T}^{2}}{\mathcal{T}^{2}+32 \mathcal{L}^{2}} .
\end{aligned}
$$




\subsection{Multiple Scattering Random Media}

The multiple scattering random media are prepared by dispersing uniform polystyrene microspheres (Duke Standards) in plant oil suspension and kept in a quartz cuvette with thickness of $10 \mathrm{~mm}$. The oil suspension was chosen instead of aqueous suspension due to the high absorption in water of $1.55 \mu \mathrm{m}[\underline{143}]$. We used the plant oil instead of paraffin oil specific for Infrared (IR) spectroscopy as the density of plant oil $\left(0.92 \mathrm{~g} / \mathrm{cm}^{3}\right)$ is closer to the density of the scatterer $\left(1.05 \mathrm{~g} / \mathrm{cm}^{3}\right)$ [144-146]. Therefore, the scatterers suspended in plant oil will have close to neutral buoyancy and better homogeneity compared to paraffin oil. The refractive index of the oil and polystyrene microspheres are 1.47 and 1.59 respectively. We considered that the scatterers in oil suspension were isotropic and uncorrelated.

The samples prepared in this work are in weak scattering regime, where the scattering mean free path is much larger than the wavelength of the photon [147]. The mean diameters (NIST traceable) of the polystyrene microspheres that were used in this work are $0.5 \mu \mathrm{m}, 0.8 \mu \mathrm{m}, 1.6 \mu \mathrm{m}$ and $5.0 \mu \mathrm{m}$. As the polystyrene microspheres are packaged in aqueous suspension, we dilute the desired amount of polystyrene microspheres in aqueous suspension with ethanol and evaporate all the liquid solution. Then, the remaining polystyrene microspheres were mixed with the oil. Before each measurement was made, the mixture was carefully stirred to ensure the polystyrene microspheres were well dispersed and avoid trapped air bubbles in the sample. The presence of trapped air bubbles will cause large fluctuation in photon 
counting statistic, therefore induces significant uncertainty in the measurements. We used different scatterer diameters with different concentrations for changing the mean free-path while keeping the total loss of $3 \mathrm{~dB}$ (including free space to fiber coupling loss). We explored bi-partite quantum correlation and interference with one of the photon-pair in the random medium and compare the results obtained from the standard loss of $3 \mathrm{~dB}$ (neutral density filter).

In order to achieve $3 \mathrm{~dB}$ loss for each samples, we prepare random media with concentration of $1.22 \times 10^{14} \mathrm{~m}^{-3}, 1.13 \times 10^{14} \mathrm{~m}^{-3}, 0.58 \times 10^{14} \mathrm{~m}^{-3}$, and $0.08 \times 10^{14}$ $\mathrm{m}^{-3}$ for scatterers with diameters $\left(\phi_{1,2,3,4}\right)$ of $0.5 \mu \mathrm{m}, 0.8 \mu \mathrm{m}, 1.6 \mu \mathrm{m}$ and $5.0 \mu \mathrm{m}$ Thess corresponds to the scattering mean free-path $\left(\ell_{1,2,3,4}\right)$ of $0.019 \mathrm{~m}, 0.010 \mathrm{~m}$, $0.004 \mathrm{~m}$, and $0.003 \mathrm{~m}$, respectively. The full-width-half-maximum (FWHM) of the scattering angle for all samples was measured to be around $6^{\circ}-7.4^{\circ}$. It is noted that the random medium with scatterer in smaller diameter tends to scatter light over broader angle than the larger one, and reduced the coupling efficiency from free space and fiber. In our experiment, the scattering polystyrene spheres in oil suspension, were subject to Brownian motion [148]. However, the statistical fluctuation due to the Brownian motion is negligible due to the long integration time of the single photon count and coincidence count. The properties of the random medium samples that prepared in our experiment are listed in Table 5.1. 
Table 5.1 Properties of the random medium samples

\begin{tabular}{|l|c|c|c|c|}
\hline & Sample 1 & Sample 2 & Sample 3 & Sample 4 \\
\hline Scatter diameter $(\mu \mathrm{m})$ & 0.5 & 0.8 & 1.6 & 5 \\
\hline Concentration $\left(/ \mathrm{m}^{3}\right)$ & $1.22 \times 10^{14}$ & $1.13 \times 10^{14}$ & $0.58 \times 10^{14}$ & $0.08 \times 10^{14}$ \\
\hline Mean free path $(\mathrm{m})$ & 0.019 & 0.010 & 0.004 & 0.003 \\
\hline Scattering angle $\left(^{\circ}\right)$ & 7.4 & 7.3 & 6.7 & 6.0 \\
\hline
\end{tabular}

\subsection{Experiment Setup}

The correlated and polarization-entangled photon pair was created via spontaneous four wave mixing by adopting a compact counter propagating scheme (CPS) using $10 \mathrm{~m}$ of highly nonlinear fiber (HNLF) as described in chapter 4.

As shown in Fig. 5.2, the photon-pair emerging from the CPS was separated by cascaded dense wavelength division multiplexing (DWDM) filters with $1 \mathrm{~nm}$ bandwidth at $1560.6 \mathrm{~nm}$ (idler) and $1547.7 \mathrm{~nm}$ (signal), providing the pump pulse suppression of more than $100 \mathrm{~dB}$. The insertion losses of cascaded DWDM for signal and idler bands were measured to be about $0.9 \mathrm{~dB}$ each. The outputs photons from the signal channel were guided through the polarization analyzers consisting of a quarterwave plate, a half-wave plate and a polarizing beam splitter. The collimated idler photon was sent through similar setup with neutral density filter or random medium inserted before the PBS. The combination of a quarter-wave plate and a half-wave 
plate was used to compensate for the birefringence of coherent component of the idler photon.

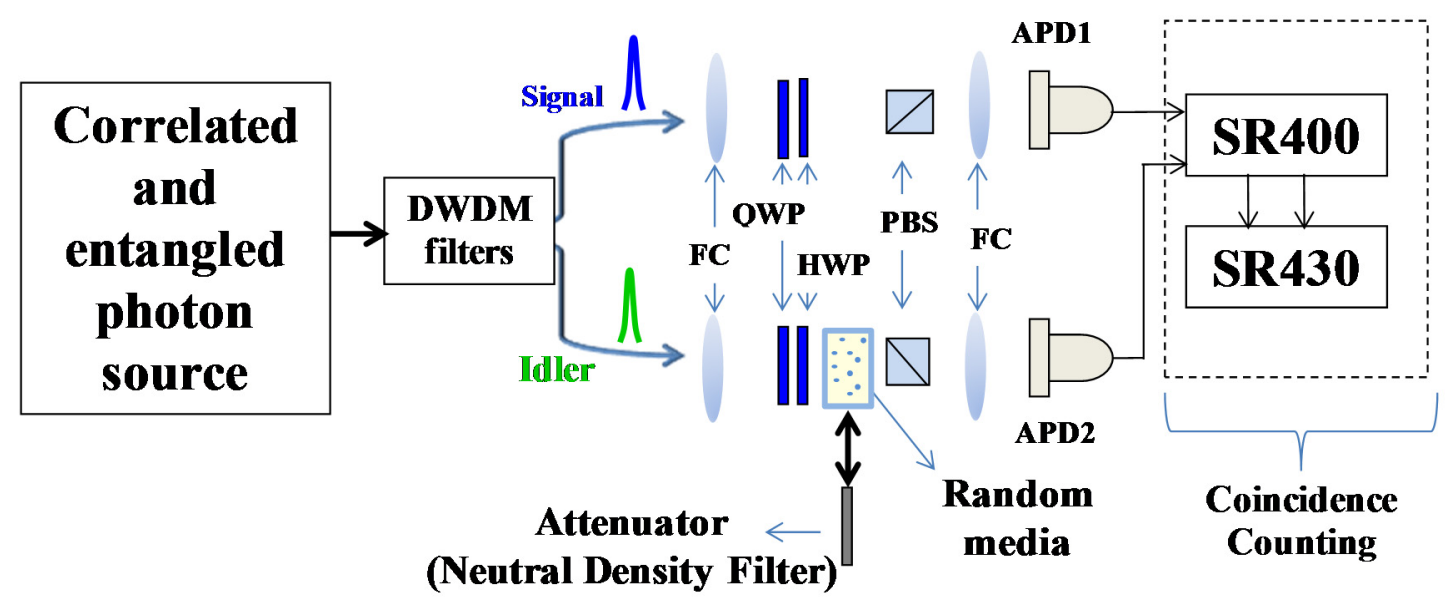

Figure 5.2. Experiment setup for measuring CAR and two-photon interference of the signal photon in a normal channel and the idler photon experiencing multiple scattering events. FC (fiber-to-free space collimators); PBS (polarization beam splitter); HWP and QWP (half- and quarter-wave plates); DWDM (dense wavelength division multiplexer); APD (Avalanche photodiode).

We first prepared and measured the purity of polarizationcorrelated/polarization-entangled photon-pair with attenuation in idler channel. We investigated the effect of standard loss on the photon-pair. In addition, we investigated the propagation of polarization-correlated/polarization-entangled photon pair through a multiple scattering random medium. The neutral density filter in idler channel was replaced with a random medium sample. Further details for the preparation of the polarization-correlated/polarization-entangled have been discussed in section 4.6. 
The scattered photons emerging from the random medium were collected by fiber-to-free space collimators ( $\mathrm{NA}=0.25)$, which were placed closely right after the PBS. Considering the effect of the constant loss on the quantum correlation of the photon pair, we made sure the attenuation (about $3 \mathrm{~dB}$ ) of ballistic beam is almost similar for all scattering samples. The coupling efficiency of the fiber-to-free space collimator was included for attenuation measurement.

Both signal and idler photons were detected by fiber coupled InGaAs/InP avalanche photodiodes operated in gated Geiger mode at room temperature.

\subsection{Results}

\subsubsection{Standard Loss}

For the polarization-correlated photon-pair, we measured the Coincidence to accidental-coincidence ratio (CAR) with the idler photon propagating through the neutral density filter with attenuation of $1 \mathrm{~dB}, 3 \mathrm{~dB}$ and $5 \mathrm{~dB}$. In CAR measurement, the polarization analyzer was oriented so that the co-polarized (horizontally) signal and idler photons will pass through to APD1 and APD2. The error bars of the plots are derived from Poisson statistic error of the single photon detection. The intrinsic dark count of single photon detectors and its fluctuation contributed to the size of the error bar. 
At different attenuations, we measured the CAR as a function of average pump power as shown in Fig. 5.3. The maximum CAR value for attenuation of $1 \mathrm{~dB}$, $3 \mathrm{~dB}$ and $5 \mathrm{~dB}$ at idler channel were equal to 26,23 , and 16 respectively.

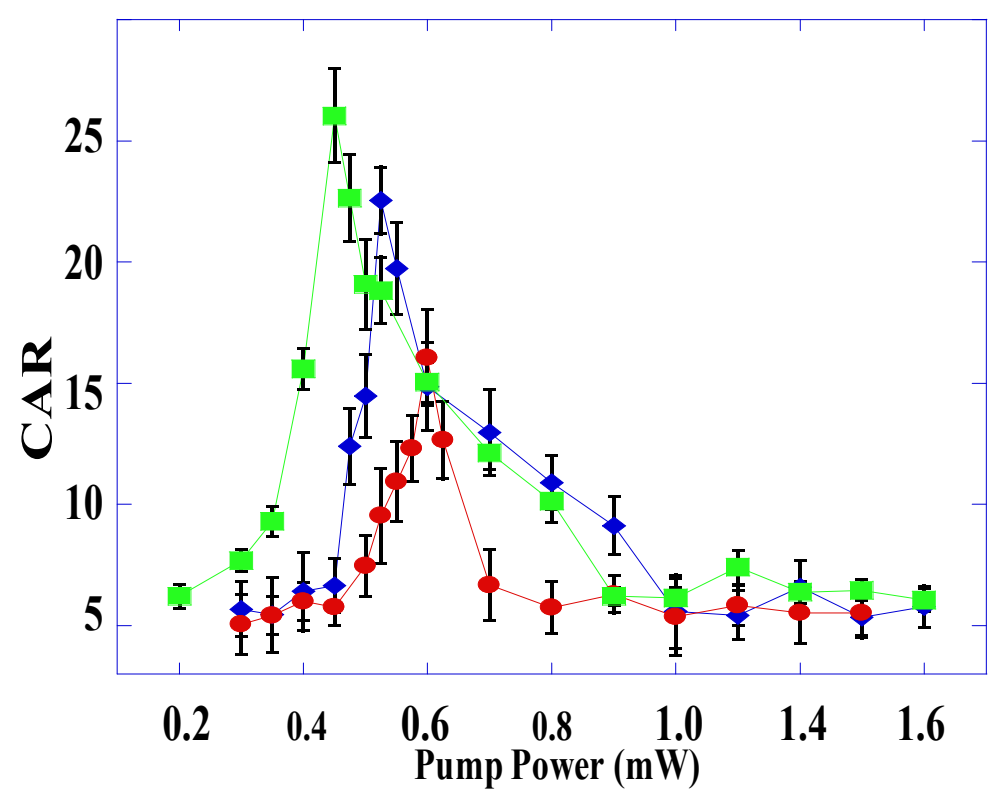

Figure 5.3. The Coincidence to accidental coincidence ratio (CAR) versus pump power with different attenuations. (Green square $=1 \mathrm{~dB})$, (Blue diamond $=3 \mathrm{~dB})$ and $($ Red dot $=5 \mathrm{~dB})$.

At higher standard loss, higher pump power was needed to achieve maximum CAR value. This is mainly because more photon counts were needed to compensate the loss and accumulate significant photon counts above the intrinsic dark count of single photon detectors. The predicted visibility of correlated photon pair for different standard losses can be calculated from CAR measurement and is given as,

$$
\mathcal{V}_{\text {cor }}=\frac{C C-A C}{C C+A C}=\frac{C A R_{\max }-1}{C A R_{\max }+1}
$$

$\mathcal{V}_{c o r}$ is the predicted visibility of two photon interference when the correlated photon pair was used to generate polarization-entangled state. The $\mathcal{V}_{c o r}$ of correlated photon- 
pair for attenuation of $1 \mathrm{~dB}, 3 \mathrm{~dB}$ and $5 \mathrm{~dB}$ in idler channel were calculated to be 92.6\%, 91.4\% and 88.2\% as depicted in Fig. 5.4. The observed decreasing maximum CAR value as a function of attenuation is shown in Fig. 5.4. It proves that standard loss in transmission channel degrades the quality of correlated photon pair.

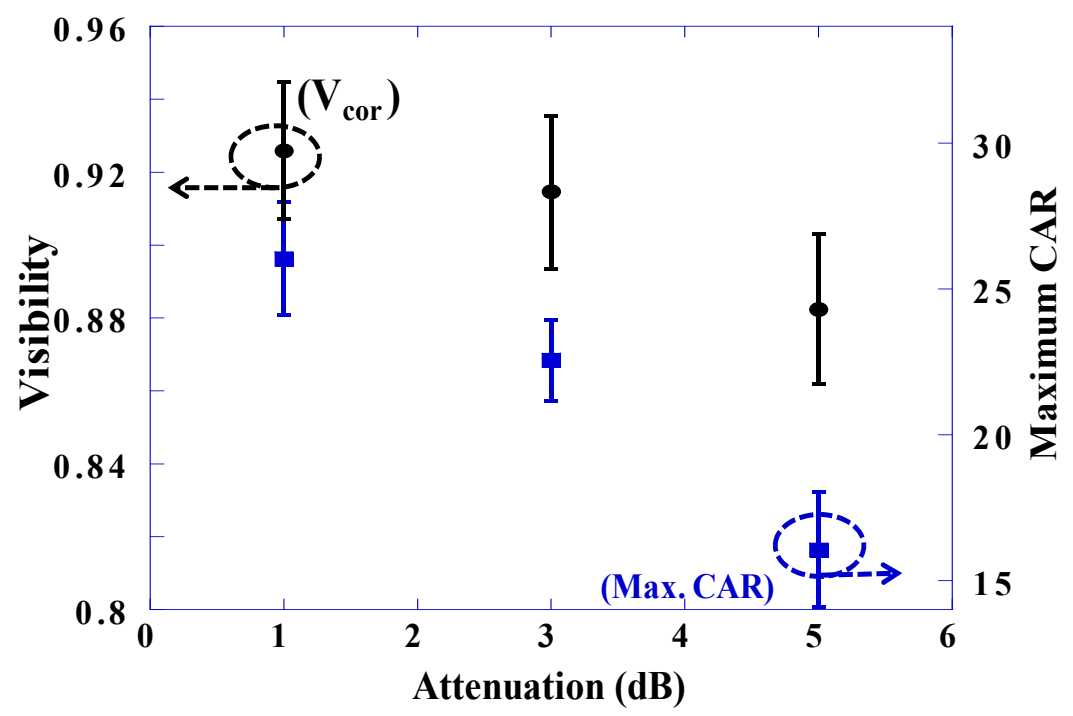

Figure 5.4. The measured CAR estimates visibility (Blue box) and maximum CAR (Solid circle) as a function of standard loss.

We then prepared the polarization entangled state $\left|\Psi_{\text {ent }}\right\rangle=\frac{1}{\sqrt{2}}\left[\left|H_{i} H_{S}\right\rangle+\right.$ $\left.\left|V_{i} V_{s}\right\rangle\right]$ and measured the two-photon interference (TPI) as a function of relative analyzer polarization angle of signal-idler photons. A typical two-photon interference plot as a function of relative analyzer polarization angle is shown in Fig. 5.5. For polarization-entangled photon pair, TPI's visibility is defined by

$$
\mathcal{V}_{\text {ent }}=\frac{C C_{\max }-C C_{\min }}{C C_{\max }+C C_{\min }}
$$

where $C C_{\max }$ is maximum coincidence and $C C_{\min }$ is the minimum coincidence in the TPI plot. 


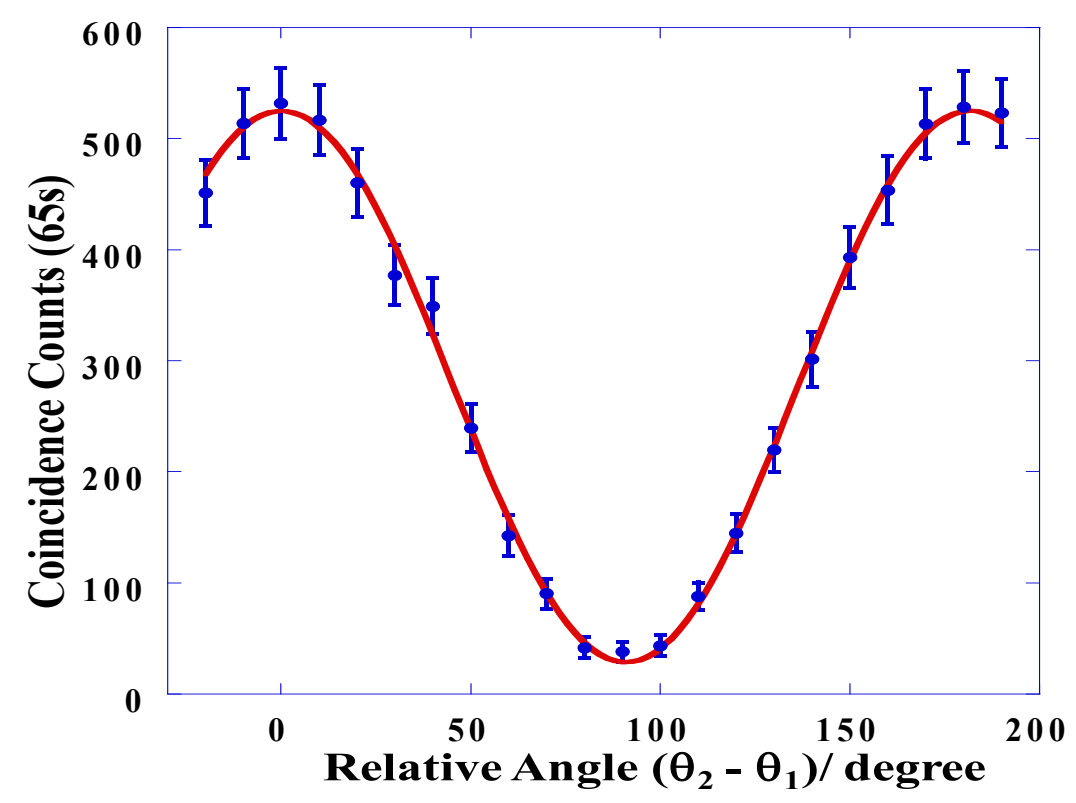

Figure 5.5. Two-photon interference fringes as a function of analyzer relative angle for the standard loss of $5 \mathrm{~dB}$ with HNLF at $300 \mathrm{~K}$. (Blue dot) and (ii) $\theta_{1}=-45^{\circ}$ (Red dot). The solid lines are the theoretical curve fitting.

The measured visibility for polarization entangled photon pair $\mathcal{V}_{\text {ent }}$ for attenuation of $1 \mathrm{~dB}, 3 \mathrm{~dB}$ and $5 \mathrm{~dB}$ were $93.3 \%, 91.8 \%$ and $89.1 \%$, respectively. We found that $\mathcal{V}_{\text {ent }}$ and $\mathcal{V}_{\text {cor }}$ are in good agreement for each attenuation. This implies that quantum correlation and interference for both polarization correlated and entangled photon pair are equally sensitive to the standard losses in transmission channel. 


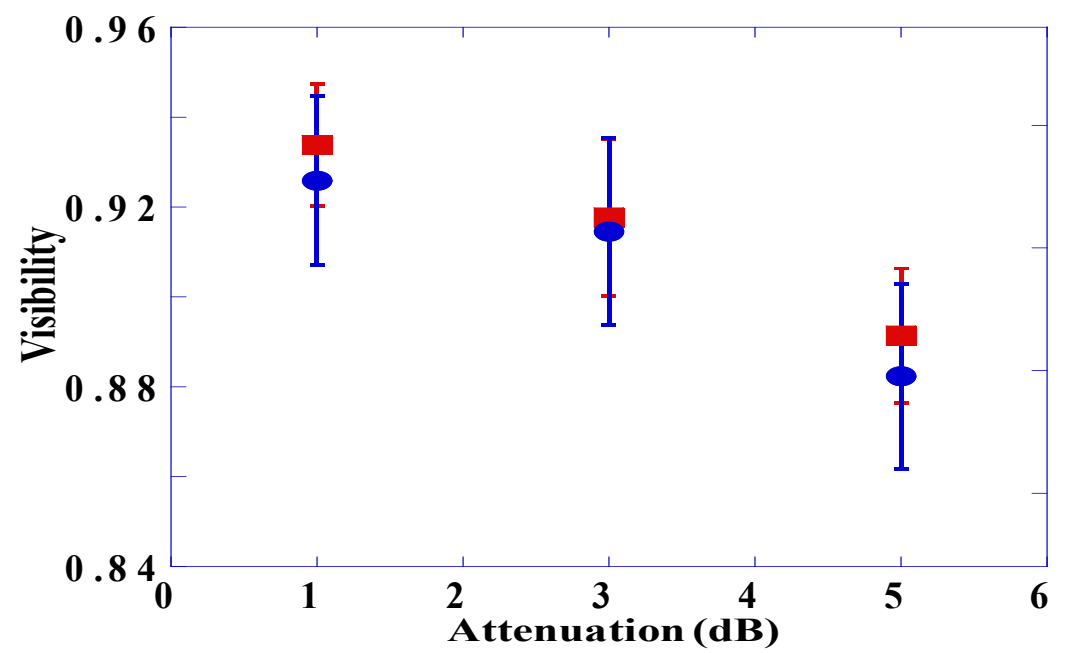

Figure 5.6. The measured two-photon interference visibility $\mathcal{V}_{\text {ent }}$ (Red box) and predicted visibility $\mathcal{V}_{\text {cor }}$ (Solid circle) versus standard losses.

\subsubsection{Multiple Scattering Random Media}

We used the photon-pair generated in the HNLF at room temperature for exploring depolarization effect on the photon-pair. First, we measured CAR value as a function of pump power for different scattering mean free-paths for the horizontal polarization-correlated photon-pair with the idler photon scattering through the samples. Results of CAR measurement is shown in Fig.5.7. We obtained maximum CAR values of 20.3, 19.8, 18.3 and 16.9 for the mean free-path of path $\left(\ell_{1,2,3,4}\right)$ of $0.019 \mathrm{~m}, 0.010 \mathrm{~m}, 0.004 \mathrm{~m}$, and 0.003 respectively. The maximum CAR values decreased as the idler photon propagated through a random medium with shorter scattering mean free-path and experienced more scattering events. When neutral density filter was used at attenuation, we obtained CAR value of about 23 for a standard loss of $3 \mathrm{~dB}$, which was higher than the CAR values obtained with multiple scattering random media. This could be predicted from the Eq. 5.6. The CAR values 
with random media was lower than the CAR values obtained with standard loss (where the $\mathrm{L}=0$ ). We plotted the predicted visibility $\mathcal{V}_{\text {cor }}$ as a function of mean free path obtained from the CAR measurement in Fig. 5.8. In addition to the detection system, the Brownian motion of the random media caused the fluctuation in single photon counts and contributed to the size of error bars.

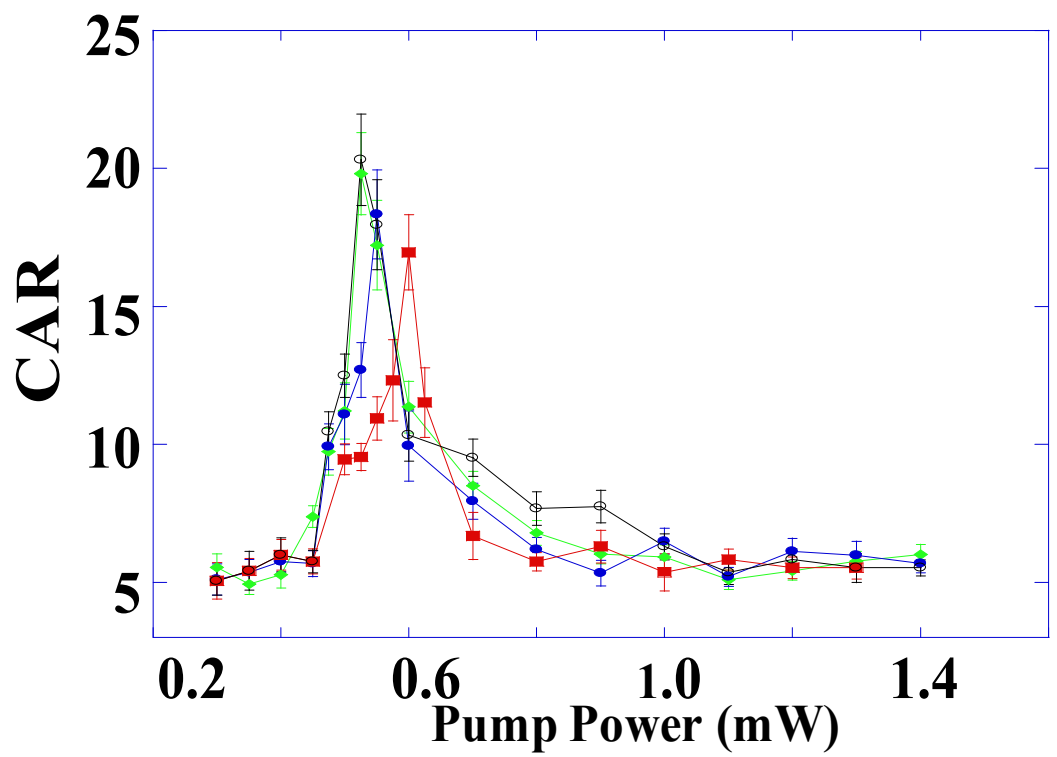

Figure 5.7. The Coincidence to accidental coincidence ratio (CAR) of correlated photon pair versus pump power for different scattering mean free path. (Black circle, $\ell=0.010 \mathrm{~m})($ Green diamond, $\ell=0.010 \mathrm{~m}$ ), (Blue $\operatorname{dot} \ell=0.004 \mathrm{~m})$ and (Red box $\ell$ $=0.004 \mathrm{~m})$. 


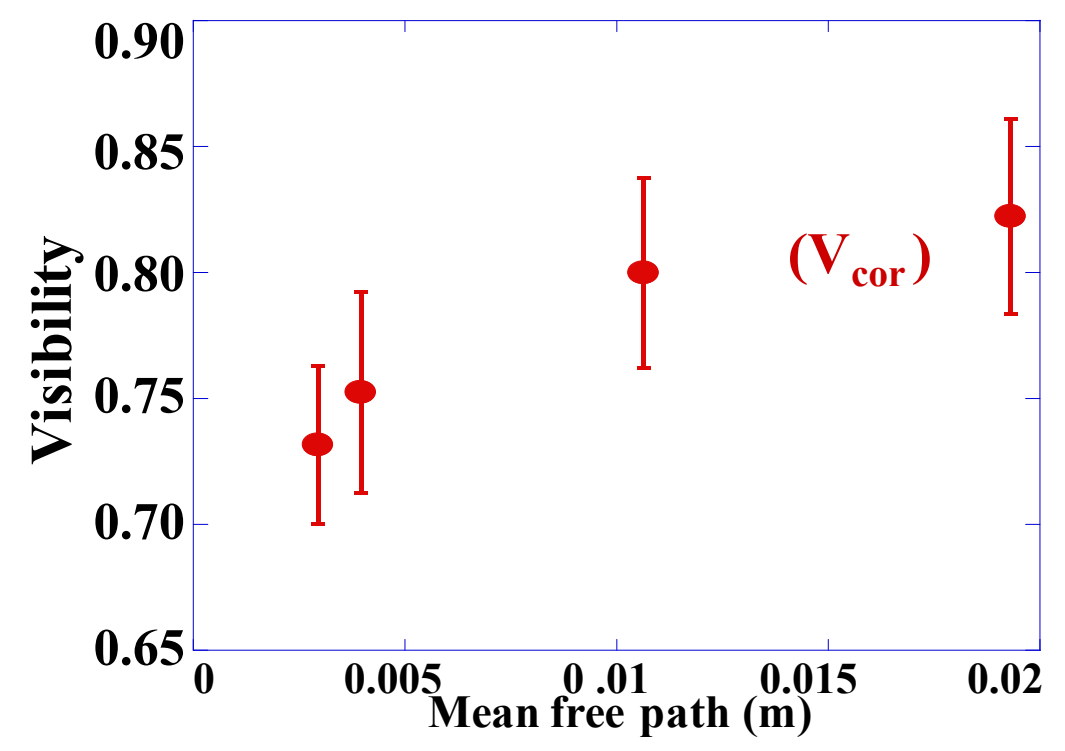

Figure 5.8. The predicted visibility for correlated photon pair, $\mathcal{V}_{\text {cor }}$ as a function of scattering mean free path.

Next, we measured two-photon interference of the polarization-entangled photon pair with the idler photon scattered by a random medium. The two-photon interference plot for the random media of $\ell=0.019 \mathrm{~m}$ is shown in Fig.5.10. We fitted the two-photon interference fringe with the Eq. 5.20 (shown as the dotted line). Two maxima conditions $\left\{\left(\theta_{2}-\theta_{1}\right)=0^{\circ}, 180^{\circ}\right\}$ fall within in the error bars of our experimental data. We then obtained the $\mathcal{V}_{\text {ent }}$, or the average visibility as discussed in Eq. 5.26. We repeated the measurement of two-photon interference for all samples and plot the visibility as a function of scattering mean free-path in Fig.5.10. Similar to the $\mathcal{V}_{\text {cor }}$, it was observed that $\mathcal{V}_{\text {ent }}$ was increasing with the scattering mean free path of the random media. 


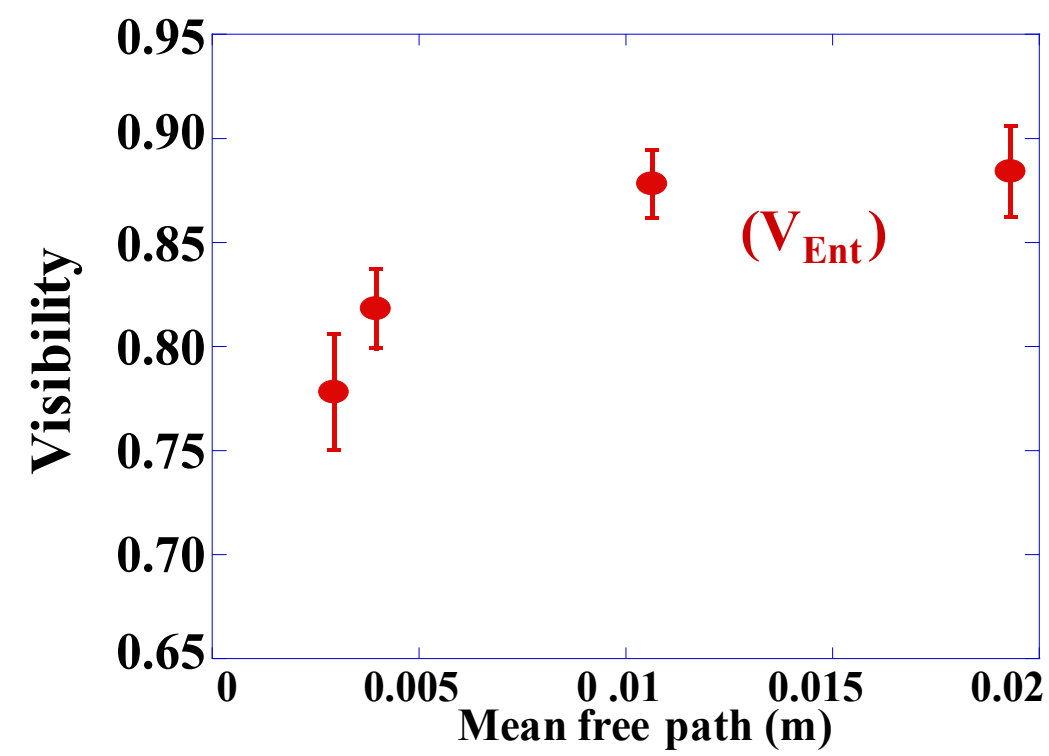

Figure 5.9. The $\mathcal{V}_{\text {ent }}($ Red dot) versus scattering mean free path.

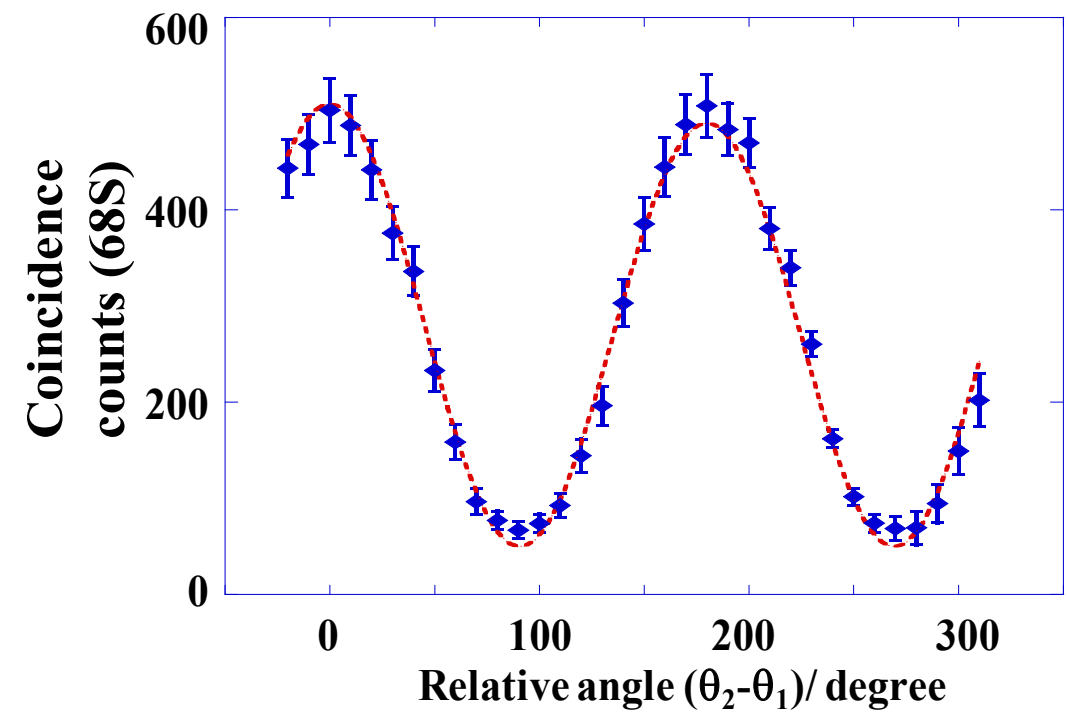

Figure 5.10. Two-photon interference fringes (Blue square) as a function of relative polarization angle, the dotted red line are curve fitting. Coincidence counts was accumulated for 68 seconds. 


\subsubsection{Influence of Raman photons in Fiber Source}

In order to identify and separate the Raman photon from the noise photon induced by depolarization of the idler photon, we reduced the Raman photon by cooling the HNLF to $77 \mathrm{~K}$. To identify and separate the Raman photon, we analyzed CAR measurement for the standard loss of $3 \mathrm{~dB}$ and the scattering medium $(\ell=0.019 \mathrm{~m}, \phi$ $=0.5 \mu \mathrm{m})$ with the HNLF source at $300 \mathrm{~K}$ and $77 \mathrm{~K}$. In this experiment, similar average pump power of $0.5 \mathrm{~mW}$ was used.

For the standard loss of $3 \mathrm{~dB}$ and average pump power of $0.5 \mathrm{~mW}$, the $\mathrm{CAR}_{3 \mathrm{~dB}}$ values were $14.1(300 \mathrm{~K})$ and $56.5(77 \mathrm{~K})$. For the scattering medium $(\ell=$ $0.019 \mathrm{~m}, \phi=0.5 \mu \mathrm{m})$, the $\mathrm{CAR}_{\mathrm{RM}}$ values were $12.8(300 \mathrm{~K})$ and $48.4(77 \mathrm{~K})$ respectively. Comparing the CAR values obtained for the $3 \mathrm{~dB}$ standard loss and scattering medium $(\ell=0.019 \mathrm{~m}, \phi 1=0.5 \mu \mathrm{m})$ with $\mathrm{HNLF}$ at $300 \mathrm{~K}$, the CAR value reduced to,

$$
\begin{aligned}
\left(C A R_{3 d B}\right)_{300 K}-\left(C A R_{R M}\right)_{300 K} & =14.1-12.8 \\
& =1.3 .
\end{aligned}
$$

The reduction in CAR value in scattering medium is mainly due to depolarization noise photon in scattering as amount of Raman photons remain the same for both cases.. Similarly, when the HNLF in $77 \mathrm{~K}$ the CAR reduced to

$$
\left(C A R_{3 d B}\right)_{77 K}-\left(C A R_{R M}\right)_{77 K}=56.5-48.4
$$




$$
=8.1
$$

One can see that the reduction in CAR is about 8.0 with the HNLF at $77 \mathrm{~K}$.

From the results that were obtained at both temperatures, we investigated the contribution of Raman photon and depolarization photon on CAR values in more detail. We denote the contribution of Raman noise photon by substitute "1" and denote the contribution of the depolarization photon as $X_{1}$. The reduction of the CAR value for the scattering medium compared to standard loss can be written as,

$$
\left(C A R_{R M}\right)=\frac{\left(C A R_{3 d B}\right)}{1+X_{1}}
$$

From the CAR value measurement with HNLF at $300 \mathrm{~K}$, the Eq. 5.29 is equal to

$$
\left(C A R_{R M}\right)_{300 K}=\frac{\left(C A R_{3 d B}\right)_{300 K}}{1+X_{1}}=\frac{14.1}{1+X_{1}}=12.8
$$

where we can solve for $x_{1}=0.1$. The contribution of depolarization noise photon was about 10 times smaller than the Raman photon. When the HNLF was cooled to $77 \mathrm{~K}$, the Raman photons were reduced by a factor of $4[\underline{98}, \underline{131}]$. On the other hand, the contribution of depolarization noise photon was expected to remain the same. Substituting the CAR results with HNLF at $77 \mathrm{~K}$ into Eq. 5.29 one obtains

$$
\left(C A R_{R M}\right)_{77 K}=\frac{\left(C A R_{3 d B}\right)_{77 K}}{1+X_{1}^{\prime}}=\frac{56.5}{1+X_{1}^{\prime}}=48.4
$$

where, $X_{1}^{\prime}=0.04$, which indicates contribution of depolarization photon is reduced as well. The presence of Raman noise photon in photon-pair source before entering a 
random medium enhanced the depolarization effect. Comparing $X_{1}^{\prime}$ and $X_{1}$, we can calculate the reduction factor of the depolarization photon at $77 \mathrm{~K}$ as ,

$$
\frac{x_{1}}{x_{1}^{\prime}}=2.4
$$

Our observation proved that the purity of the photon-pair source plays an important role in increasing and decreasing depolarization effect by scattering process.

\subsection{Discussions}

The $\mathcal{V}_{\text {ent }}$ and $\mathcal{V}_{\text {cor }}$ as a function of mean free path are plotted in Fig. 5.11. First, we observed that TPI's visibility $\mathcal{V}_{\text {ent }}$ is better than the visibility $\mathcal{V}_{\text {cor }}$ obtained from the CAR measurement. Also shown in Fig. 5.11 is TPI's visibility of $91.8 \%$ for a standard loss of $3 \mathrm{~dB}$, which is higher than both $\mathcal{V}_{\text {cor }}$ and $\mathcal{V}_{\text {ent }}$. This is in conjunction to the observation in standard loss as shown in Fig. 5.7, where the CAR estimate visibility agrees with the measured TPI's visibility. The fitting curves of the visibility $\mathcal{V}_{\text {cor }}$ and $\mathcal{V}_{\text {ent }}$ are obtained from Eq. 5.17 and Eq. 5.26, respectively. Quantum correlation of polarization entangled photon pair was better preserved than polarization-correlated photon-pair as one of the photon-pair experiences random scattering process in the random medium. From the fitting of Eq. 5.17 and Eq. 5.26, we obtained the average of transmission amplitude $\mathcal{T}=0.77$ i.e. $\mathcal{T}^{2}=0.6$. With the approximation $80 \%$ of photons were coupled into the fiber, we have $0.8 \times 0.6=0.48$, which is close to $3 \mathrm{~dB}$ loss that we claimed for all samples. 


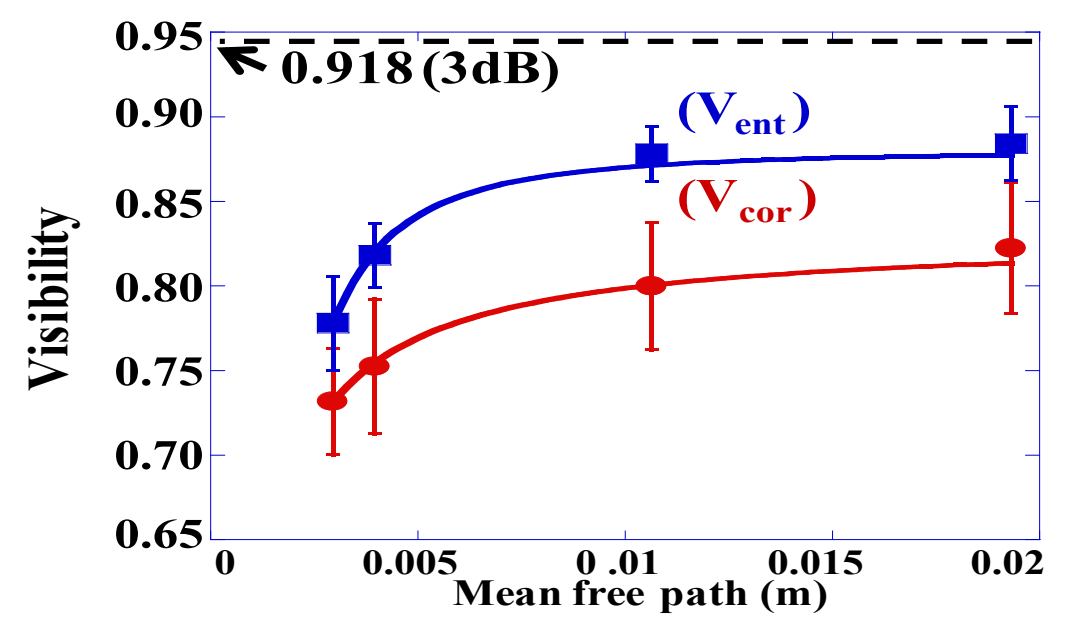

Figure 5.11. The $\mathcal{V}_{\text {ent }}$ (Blue square) and $\mathcal{V}_{\text {cor }}(\operatorname{Red}$ dot) versus mean free path, the solid lines are fitting curves for $\mathcal{V}_{\text {ent }}$ and $\mathcal{V}_{\text {cor }}$. The dashed line is the visibility measured with $3 \mathrm{~dB}$ standard loss.

With the similar average pump power of $0.5 \mathrm{~mW}$ and the HNLF at $300 \mathrm{~K}$, we have had the CARRM values of $11.5\left(\ell_{2}=0.010 \mathrm{~m}\right), 11.1\left(\ell_{3}=0.004 \mathrm{~m}\right)$ and $9.5\left(\ell_{4}\right.$ $=0.003 \mathrm{~m}$ ). Using the standard loss of $3 \mathrm{~dB}$ with the $\mathrm{CAR}_{3 \mathrm{~dB}}=14.1$ and CARRM for $\ell_{2,3,4}$, we calculated $X_{2,3,4}$ for each sample by using equation

$$
\left(C A R_{R M}\right)=\frac{\left(C A R_{3 d B}\right)}{1+X_{i}},(i=1,2,3,4) .
$$

We obtained $x_{2}=0.22, x_{3}=0.28$ and $x_{4}=0.48 . x_{i}$, the ratio of depolarized photon to Raman photon was higher with the sample of shorter mean free path (more scattering events). 
Since all measurements have the contributions from Raman noise photon and depolarization noise photon, we denote the experimentally measured noise amplitude as $\mathcal{L}^{\prime}$,

$$
\begin{gathered}
\mathcal{L} \rightarrow \mathcal{L}^{\prime}, \\
\mathcal{L}^{\prime 2}=\mathcal{R}^{2}+\mathcal{L}_{\mathrm{d}}^{2} .
\end{gathered}
$$

Where $\mathcal{R}$ and $\mathcal{L}_{\mathrm{d}}$ are the strength of noise amplitudes for Raman photon and depolarization photon in the random medium. Since the ratio of depolarization photon to Raman noise photon was obtained in Eq. 5.30. We can express the noise amplitude for depolarization photon as

$$
\mathcal{L}_{\mathrm{d}_{1}}=\sqrt{X_{1}} \mathcal{R}
$$

and use the Eq. 5.35 to substitute $\mathcal{R}$ in Eq. 5.34 to obtain

$$
\mathcal{L}^{\prime 2}=\left(1+\frac{1}{x_{1}}\right) \mathcal{L}_{\mathrm{d}_{1}}^{2}
$$

From the fitting curves in Fig. 5.12, where $\mathcal{L}=\frac{\mathcal{A}^{\prime}}{\ell}$, we obtained the experimentally measured depolarization coefficient $\mathcal{A}^{\prime}=\mathcal{L}_{1,2,3,4}^{\prime} \ell_{1,2,3,4}=0.0003 \mathrm{~m}$. We then extract the depolarization constant associated with the noise operator $\hat{c}$, which is originated from the multiple scattering for the sample with scatter diameter $(\ell=0.019 \mathrm{~m}, \phi=0.5 \mu \mathrm{m})$ given as

$$
\mathcal{A}_{1}=\mathcal{L}_{\mathrm{d}_{1}} \ell_{1}
$$


Substituting $\mathcal{L}_{\mathrm{d}_{1}}$ in Eq. 5.36 and $\ell_{1}=\frac{\mathcal{A}^{\prime}}{\mathcal{L}^{\prime}}$, we get

$$
\begin{aligned}
\mathcal{A}_{1} & =\frac{\mathcal{L}^{\prime}}{\sqrt{1+\frac{1}{x_{1}}}} \cdot \frac{\mathcal{A}^{\prime}}{\mathcal{L}^{\prime}} \\
& =\frac{\mathcal{A}^{\prime}}{\sqrt{1+\frac{1}{x_{1}}}}=9.0 \times 10^{-5} \mathrm{~m}
\end{aligned}
$$

Now with the $\mathcal{A}_{1}=9.0 \times 10^{-5} \mathrm{~m}$, we can also calculate $\mathcal{L}_{\mathrm{d}_{1}}=0.0047$ from Eq. 5.37 .

Similarly, we can obtain $\mathcal{A}_{2}=12.0 \times 10^{-5} \mathrm{~m}, \mathcal{A}_{3}=14.0 \times 10^{-5} \mathrm{~m}$, and $\mathcal{A}_{4}=17.0 \times 10^{-5} \mathrm{~m}$, for the scattering mean free-path $\ell_{2,3,4}$ corresponding to the depolarization amplitude of $\mathcal{L}_{\mathrm{d}_{2}}=0.012, \mathcal{L}_{\mathrm{d}_{3}}=0.035$, and $\mathcal{L}_{\mathrm{d}_{4}}=0.056$, respectively. Our results showed that the idler photon is less depolarized in the medium with larger scattering mean free-path (fewer scattering events).

Table 5.2 Summary of the results for the scattering random media.

\begin{tabular}{|c|c|c|c|c|}
\hline & $\begin{array}{c}\text { Sample 1 } \\
(0.5 \mu \mathrm{m})\end{array}$ & $\begin{array}{c}\text { Sample 2 } \\
(0.8 \mu \mathrm{m})\end{array}$ & $\begin{array}{c}\text { Sample 3 } \\
(1.6 \mu \mathrm{m})\end{array}$ & $\begin{array}{c}\text { Sample 4 } \\
(5.0 \mu \mathrm{m})\end{array}$ \\
\hline$\ell(\mathrm{m})$ & 0.019 & 0.010 & 0.004 & 0.003 \\
\hline $\mathcal{V}_{\text {ent }}(\%)$ & 88.4 & 87.8 & 81.7 & 77.9 \\
\hline $\mathcal{V}_{\text {cor }}(\%)$ & 82.2 & 80.0 & 75.2 & 73.1 \\
\hline Maximum CAR & 20.3 & 19.8 & 18.3 & 16.9 \\
\hline $\mathcal{X}_{i}$ & 0.1 & 0.22 & 0.28 & 0.48 \\
\hline $\mathcal{A}_{\mathrm{i}}(\mathrm{m})$ & $9 \times 10^{-5}$ & $1.2 \times 10^{-4}$ & $1.4 \times 10^{-4}$ & $1.7 \times 10^{-4}$ \\
\hline $\mathcal{L}_{\mathrm{d}_{i}}$ & 0.0047 & 0.012 & 0.035 & 0.056 \\
\hline
\end{tabular}




\section{Chapter 6}

\section{Summary and Outlook}

In this dissertation, we have explored quantum correlations of single photons, weak coherent states, and polarization-correlated/-entangled photons in macroscopic environments. This included: macroscopic mirrors, spatially separated observers, noisy photons sources and propagation medium with loss or disturbances.

In chapter 2, we proposed a measurement scheme for observing quantum correlations and entanglement. The experiment measured spatial properties of two macroscopic mirrors using single photons spatial compass states. Two spatial versions of compass states were generated by single photons in a simple interferometer. The single photons were in single Gaussian mode. Wave-particle duality characteristic of the Wigner function was used to characterize spatial compass state in phase space. The chessboard pattern of spatial compass states determined the sensitivity for measuring the displacement and tilt of the mirrors. The proposed imaging system could measure displacement and tilt correlations of two mirrors under real experimental condition. A single photon detector and a squarer were needed to measure the interference of two spatial compass states, and then obtain the propensity $\mathcal{P}_{b}$. One of the compass states operates as detected state and another one 
as filtering state. Variances in position and momentum of the proposed imaging system were calculated. The EPR entanglement regions were visualized in propensity plot. In addition, we formulated the discrete-like properties of the propensity $\mathcal{P}_{b}\left(d_{x}, d_{p}\right)$, where the correlation spots were identified by a pair of discrete number $(m, n)$. The discrete correlation spots in $\mathcal{P}_{b}(m, n)$ can be used to explore environmental perturbed quantum jumps of the EPR correlations in phase space.

Our results showed that variances in position and momentum are much smaller than standard quantum limit when using a Gaussian TEM00 beam [이]. The potential application of the proposed imaging system could be quantum-enhanced metrology for macroscopic objects, such as the test mass for graviton detection. In addition, the proposed imaging system can be used to observe macroscopic entanglement. We can cool one mirror and use it as a reference for the other mirror that is coupled to ambient environment. Then propensity $\mathcal{P}_{b}\left(d_{x}, d_{p}\right)$ measurement can be used to determine whether the mirrors correlation satisfies the EPR criterion for entanglement. Since the propensity $\mathcal{P}_{b}$ can be discretized and formulated in finitedimensional Hilbert space, correlation spots are potentially useful for demonstrating discrete phase-space quantum computing and information processing.

In chapter 3, we investigated intrinsic quantum correlations of weak coherent states. We demonstrated a proof of principle experiment in utilizing intrinsic quantum correlations of weak coherent states for quantum communication. In this work, we employed a weak local oscillator field to extract intrinsic correlations of 148 
weak coherent states between two parties using a balanced homodyne measurement. We implemented four types of bipartite correlation functions between two distant observers separated by $10 \mathrm{~km}$ optical fiber. The bipartite correlations between two observers were obtained by the product of interference signals measured by both observers. Our results revealed that information of the interference signal will be protected by the large quantum phase fluctuation. It is associated with low mean photon number fluctuation of weak coherent state. For practical quantum key distribution, we demonstrated bits correlations measurement of each bipartite correlation at detectors $\mathrm{A}$ and $\mathrm{B}$. The lock-in amplifier was used to measure quadrature phase of weak coherent state. Then, positive (negative) value of measured quadrature signal was encoded as keys/bits ' 1 ' ('0'), respectively. Every bit measurement can be the raw quantum key shared by both observers.

The realization of intrinsic quantum correlation of weak coherent state can be a stepping stone toward linear-optics quantum computing with weak coherent states. The proposed scheme can be used as a supplement to the existence decoy-state Bennett-Brassard 1984 protocol and differential phase-shift quantum key distribution (DPS-QKD) protocol. The interference signal of weak coherent states and local oscillator was concealed by quantum phase fluctuations. This could add another physical layer of security to these protocols. However, intrinsic correlation of weak coherent states does not exhibit nonlocality as compared to entangled-photon source. Therefore, classical amplification of optical signal using current available technology such as optical amplifier can easily extends the range of quantum key transmission 
[149]. Another important feature of our scheme is that only linear optics was required to establish the correlations between two observers. Hence, it is possible to implement the proposed scheme with integrated photonics circuit [150].

In chapter 4 , we explored quantum correlation and entanglement of photonpairs that exhibit quantum nonlocality. Generation of correlated and polarizationentangled photon pair at telecom wavelength using highly nonlinear fiber (HNLF) was demonstrated. We used counter propagating scheme to generate correlated and entangled photon pair in this work. We obtained optimum coincidence to accidentalcoincidence ratio (CAR) with $7 \times 10^{7}$ photons per pump pulse. We observed CAR of $29 \pm 3$ at $300 \mathrm{~K}$ and as high as $130 \pm 5$ at $77 \mathrm{~K}$. For characterization of polarizationentangled photon source, we prepared the polarization-entangled two photon state $\left|\Psi^{+}\right\rangle=\frac{1}{\sqrt{2}}\left(\left|H_{i} H_{s}\right\rangle+\left|V_{i} V_{s}\right\rangle\right)$ and measured two-photon interference (TPI) visibility. When the HNLF was at $300 \mathrm{~K}(77 \mathrm{~K})$, TPI visibility $>92 \%(>98 \%)$ was observed. Photon-pair production rate about factor 3(2) higher than using a $300 \mathrm{~m}$ dispersionshifted fiber was observed. Excellent visibility and high photon pair production rate are two crucial factors for the application of quantum key distribution. Later on, we proved the non-local behavior of polarization-entangled photon pair by violating Clauser-Horne-Shimony-Holt (CHSH) Bell's inequality. At $300 \mathrm{~K}$, Bell's inequality was violated by close to 5 standard deviations; while violation of Bell's inequality by more than 12 standard deviations was observed when the HNLF was cooled to $77 \mathrm{~K}$. 
Direct generation of entangled photon-pairs in HNLF has pointed to the great potential of global scale entanglement based quantum communication. This is due to its inherent compatibility with existing fiber-optics technologies for long-distance transmission, storage, and processing. Another interesting experiment to perform with HNLF would be the generation of broadband polarization-correlated and entangled photon-pair at telecom wavelengths. Our preliminary study showed that the $10 \mathrm{~m}$ long HNLF has the potential as an ultra broadband entangled photons source. The experimental setup to generate broadband entangled photons is similar to the setup in Fig. 4.8. However, multiple-pairs of cascaded DWDM filters are needed to fully utilize all the photon-pair at different wavelengths. So far, studies on telecom wavelengths entangled photon-pair sources are limited to narrowband operation. Broadband source of telecom wavelengths entangled photon-pairs for wavelength division multiplexing entanglement distribution will be a breakthrough in realizing multi-user quantum network. Short HNLF can cover up to $200 \mathrm{~nm}$ in wavelength, which is better than current available entangled photon source [133]. One of the limitation to achieve better performance is Raman scattering in HNLF [130]. We propose to investigate the Raman gain of HNLF for small detuning on both Stokes and anti-Stokes side of pump wavelength by using photon counting technique. The experiment can be carried out by using the CPS scheme that is shown in Fig. 4.8. The cascaded DWDM filters of the signal and idler photon being replaced with cascaded tunable optical filters. Both co-polarized and cross-polarized Raman gain at small detuning will be measured. Raman gain at different temperatures (300 K and $77 \mathrm{~K}$ ) can be measured to study the temperature dependence of Raman scattering at 
different detuning wavelength. The results from this work will provide information on the intrinsic photon noise of the HNLF based entangled photon source.

In chapter 5, we investigated the quantum correlation and interference of fiber based photon-pair (Signal and Idler) at telecom wavelengths. One photon of a photon pair experienced standard loss or multiple scattering in a random medium. We proposed a semi-empirical model, where the depolarization amplitude $\mathcal{L}$ was included in the annihilation operator for idler photon that scattered through random medium. We derived the joint probability of two-photon detection $\mathcal{P}_{12}=$ $\left\langle: \hat{a}_{1}^{\dagger} \hat{a}_{2}^{\dagger}, \hat{a}_{2^{\prime}} \hat{a}_{1}:\right\rangle$ for both polarization-correlated/-entangled two-photon state. We discussed on how the visibilities, $\mathcal{V}_{\text {ent }}$ and $\mathcal{V}_{\text {cor }}$ were associated with transmission amplitude $\mathcal{T}$ and depolarization amplitude $\mathcal{L}$ of scattered photon in random medium. In our experiment, we measured joint probability of two-photon by the means of coincidence detection. We found that $\mathcal{V}_{\text {ent }}$ and $\mathcal{V}_{\text {cor }}$ were decreasing as a function of attenuation; this proved that standard loss in transmission channel was degrading quantum correlation of the photon pair. As loss is almost inevitable, the development of quantum repeater in telecommunication wavelength is likely to hold the key for long distance quantum communication [151]. Furthermore, we observed that $\mathcal{V}_{\text {ent }}$ and $\mathcal{V}_{\text {cor }}$ were decreasing with shorter scattering mean free path of the random media. Our results also proved that quantum correlation of polarization entangled photon pair is better preserved than polarization-correlated photon-pair. Therefore, entangled photon pair will be a better candidate for free space long distance quantum key distribution compared to correlated photon-pairs. Our results also showed that 
Raman photon noise will contribute to the depolarization effect in scattering process, thus increase the accidental coincidence count. Hence, the purity of two-photon state is crucial for entanglement based QKD such as Eckert 91 protocol. 


\section{Reference list:}

[1] K. Stannigel, P. Komar, S. J. M. Habraken, S. D. Bennett, M. D. Lukin, P. Zoller, and P. Rabl, Physical Review Letters 109, 013603 (2012).

[2] M. Saffman, T. G. Walker, and K. Mølmer, Reviews of Modern Physics 82, $2313(2010)$.

[3] M. Ortner, Y. L. Zhou, P. Rabl, and P. Zoller, Quantum Inf Process 10, 793 (2011).

[4] K. Sato et al., Journal of Materials Chemistry 19, 3739 (2009).

[5] D. Nikos, J. G. Dylan, T. Lin, and H. Hartmut, New Journal of Physics 15, 073017 (2013).

[6] I. A. Walmsley and M. G. Raymer, Science 307, 1733 (2005).

[7] A. B. U'Ren, K. Banaszek, and I. A. Walmsley, Quantum Info. Comput. 3, $480(2003)$.

[8] P. W. Shor, (1996).

[9] L. Grover, Pramana - J Phys 56, 333 (2001).

[10] C. H. Bennett, G. Brassard, C. Crépeau, R. Jozsa, A. Peres, and W. K. Wootters, Physical Review Letters 70, 1895 (1993).

[11] D. Bouwmeester, J. W. Pan, K. Mattle, M. Eibl, H. Weinfurter, and A. Zeilinger, Nature 390, 575 (1997).

[12] G. B. C. H. Bennett, in IEEE International Conference on Computers, Systems and Signal Processing (IEEE, Bangalore, India, 1984), pp. 175.

[13] A. K. Ekert, Physical Review Letters 67, 661 (1991).

[14] H.-K. Lo, X. Ma, and K. Chen, Physical Review Letters 94, 230504 (2005).

[15] V. Scarani, H. Bechmann-Pasquinucci, N. J. Cerf, M. Dusek, N. Lutkenhaus, and M. Peev, Reviews of Modern Physics 81, 1301 (2009).

[16] X. M. Jin, C. Z. Peng, Y. J. Deng, M. Barbieri, J. Nunn, and I. A. Walmsley, Sci Rep-Uk 3 (2013).

[17] G. Brida, M. Genovese, and I. R. Berchera, Nat Photonics 4, 227 (2010). 
[18] N. K. Langford, S. Ramelow, R. Prevedel, W. J. Munro, G. J. Milburn, and A. Zeilinger, Nature 478, 360 (2011).

[19] B. Bell, S. Kannan, A. McMillan, A. S. Clark, W. J. Wadsworth, and J. G. Rarity, Physical Review Letters 111 (2013).

[20] V. Giovannetti, S. Lloyd, and L. Maccone, Nat Photonics 5, 222 (2011).

[21] Y. M. Sua, E. Scanlon, T. Beaulieu, V. Bollen, and K. F. Lee, Phys Rev A 83 (2011).

[22] K. A. Lidke, B. Rieger, T. M. Jovin, and R. Heintzmann, Biophys J 88, 346a (2005).

[23] J. B. Clark, Z. Zhou, Q. Glorieux, A. M. Marino, and P. D. Lett, Opt Express 20, 17050 (2012).

[24] R. Schnabel, N. Mavalvala, D. E. McClelland, and P. K. Lam, Nat Commun 1 (2010).

[25] F. Toscano, D. A. R. Dalvit, L. Davidovich, and W. H. Zurek, Phys Rev A 73 (2006).

[26] M. Busshardt and M. Freyberger, Epl-Europhys Lett 96 (2011).

[27] B. Lounis and M. Orrit, Reports on Progress in Physics 68, 1129 (2005).

[28] M. D. Eisaman, J. Fan, A. Migdall, and S. V. Polyakov, Rev Sci Instrum 82 (2011).

[29] K. Inoue, E. Waks, and Y. Yamamoto, Physical Review Letters 89, 037902 (2002).

[30] M. Sasaki et al., Opt Express 19, 10387 (2011).

[31] R. Ursin et al., Nat Phys 3, 481 (2007).

[32] P. W. Milonni, J. H. Carter, C. G. Peterson, and R. J. Hughes, J Opt BQuantum S O 6, S742 (2004).

[33] A. A. Semenov and W. Vogel, Phys Rev A 80 (2009).

[34] W. H. Zurek, Astrophys J 370, 474 (1991).

[35] W. H. Zurek, Reviews of Modern Physics 75, 715 (2003). 
[36] J. R. Friedman, V. Patel, W. Chen, S. K. Tolpygo, and J. E. Lukens, Nature 406, 43 (2000).

[37] V. B. Braginsky, Y. I. Vorontsov, and K. S. Thorne, Science 209, 547 (1980).

[38] C. M. Caves, K. S. Thorne, R. W. P. Drever, V. D. Sandberg, and M. Zimmermann, Reviews of Modern Physics 52, 341 (1980).

[39] D. Kleckner, I. Pikovski, E. Jeffrey, L. Ament, E. Eliel, J. van den Brink, and D. Bouwmeester, New Journal of Physics 10 (2008).

[40] H. Muller-Ebhardt, H. Rehbein, R. Schnabel, K. Danzmann, and Y. B. Chen, Physical Review Letters 100 (2008).

[41] M. C. Teich and B. E. A. Saleh, Phys Today 43, 26 (1990).

[42] X. S. Ma et al., Nature 489, 269 (2012).

[43] X. S. Ma, S. Kropatschek, W. Naylor, T. Scheidl, J. Kofler, T. Herbst, A. Zeilinger, and R. Ursin, Opt Express 20, 23126 (2012).

[44] D. M. Greenberger, M. A. Horne, A. Shimony, and A. Zeilinger, American Journal of Physics 58, 1131 (1990).

[45] J. W. Pan, D. Bouwmeester, M. Daniell, H. Weinfurter, and A. Zeilinger, Nature 403, 515 (2000).

[46] D. Bouwmeester, J.-W. Pan, M. Daniell, H. Weinfurter, and A. Zeilinger, Physical Review Letters 82, 1345 (1999).

[47] M. Murao, D. Jonathan, M. B. Plenio, and V. Vedral, Phys Rev A 59, 156 (1999).

[48] J. G. Rarity and P. R. Tapster, Phys Rev Lett 64, 2495 (1990).

[49] Z. Y. Ou, X. Y. Zou, L. J. Wang, and L. Mandel, Phys Rev Lett 65, 321 (1990).

[50] P. G. Kwiat, E. Waks, A. G. White, I. Appelbaum, and P. H. Eberhard, Phys Rev A 60, R773 (1999).

[51] J. T. Barreiro, N. K. Langford, N. A. Peters, and P. G. Kwiat, Physical Review Letters 95, 260501 (2005).

[52] A. Einstein, B. Podolsky, and N. Rosen, Physical Review 47, 777 (1935).

[53] N. Bohr, Physical Review 48, 696 (1935). 
[54] J. S. Bell, Speakable and unspeakable in quantum mechanics : collected papers on quantum philosophy (Cambridge University Press, Cambridge Cambridgeshire ; New York, 1987).

[55] J. F. Clauser, M. A. Horne, A. Shimony, and R. A. Holt, Physical Review Letters 23, 880 (1969).

[56] A. Aspect, J. Dalibard, and G. Roger, Physical Review Letters 49, 1804 (1982).

[57] A. Aspect, P. Grangier, and G. Roger, Physical Review Letters 47, 460 (1981).

[58] P. G. Kwiat, K. Mattle, H. Weinfurter, A. Zeilinger, A. V. Sergienko, and Y. Shih, Physical Review Letters 75, 4337 (1995).

[59] K. Wodkiewicz, Physical Review Letters 52, 1064 (1984).

[60] W. H. Zurek, Phys Today 44, 36 (1991).

[61] M. Schlosshauer, Reviews of Modern Physics 76, 1267 (2005).

[62] V. Giovannetti, S. Lloyd, and L. Maccone, Physical Review Letters 96, 010401 (2006).

[63] W. H. Zurek, Nature 412, 712 (2001).

[64] D. A. R. Dalvit, R. L. De Matos, and F. Toscano, New Journal of Physics 8 (2006).

[65] A. Nunnenkamp, K. Børkje, and S. M. Girvin, Physical Review Letters 107, 063602 (2011).

[66] S. Gigan et al., Nature 444, 67 (2006).

[67] S. Groblacher, J. B. Hertzberg, M. R. Vanner, G. D. Cole, S. Gigan, K. C. Schwab, and M. Aspelmeyer, Nat Phys 5, 485 (2009).

[68] H. Müller-Ebhardt, H. Rehbein, R. Schnabel, K. Danzmann, and Y. Chen, Physical Review Letters 100, 013601 (2008).

[69] V. Giovannetti, S. Lloyd, and L. Maccone, Science 306, 1330 (2004).

[70] V. B. Braginsky and F. Y. Khalili, Reviews of Modern Physics 68, 1 (1996).

[71] A. A. Clerk, M. H. Devoret, S. M. Girvin, F. Marquardt, and R. J. Schoelkopf, Reviews of Modern Physics 82, 1155 (2010). 
[72] K. Wódkiewicz, Physics Letters A 115, 304 (1986).

[73] R. F. Oconnell and D. F. Walls, Nature 312, 257 (1984).

[74] E. Wigner, Physical Review 40, 749 (1932).

[75] A. Kenfack and K. Zyczkowski, J Opt B-Quantum S O 6, 396 (2004).

[76] K. F. Lee, F. Reil, S. Bali, A. Wax, and J. E. Thomas, Opt. Lett. 24, 1370 (1999).

[77] E. Mukamel, K. Banaszek, I. A. Walmsley, and C. Dorrer, Opt. Lett. 28, 1317 (2003).

[78] B. J. Smith, B. Killett, M. G. Raymer, I. A. Walmsley, and K. Banaszek, Opt. Lett. 30, 3365 (2005).

[79] V. I. Tatarskiı̌, Soviet Physics Uspekhi 26, 311 (1983).

[80] E. F. Galvão, Phys Rev A 71, 042302 (2005).

[81] J. P. Dahl, H. Mack, A. Wolf, and W. P. Schleich, Phys Rev A 74 (2006).

[82] K. Anatole and Ż. Karol, Journal of Optics B: Quantum and Semiclassical Optics 6, 396 (2004).

[83] C. Iaconis and I. A. Walmsley, Opt. Lett. 21, 1783 (1996).

[84] A. Wax, S. Bali, and J. E. Thomas, Phys Rev Lett 85, 66 (2000).

[85] A. Wax, S. Bali, and J. E. Thomas, Opt. Lett. 24, 1188 (1999).

[86] F. Reil and J. E. Thomas, Physical Review Letters 95, 143903 (2005).

[87] V. Bollen, Y. M. Sua, and K. F. Lee, Phys Rev A 81, 11 (2010).

[88] K. Wódkiewicz, Physical Review Letters 52, 1064 (1984).

[89] M. D. Reid, Phys Rev A 40, 913 (1989).

[90] V. Delaubert, N. Treps, M. Lassen, C. C. Harb, C. Fabre, P. K. Lam, and H. A. Bachor, Phys Rev A 74, 053823 (2006).

[91] T. C. Li, S. Kheifets, and M. G. Raizen, Nat Phys 7, 527 (2011).

[92] T. Opatrný, V. Bužek, J. Bajer, and G. Drobný, Phys Rev A 52, 2419 (1995). 
[93] U. Leonhardt, Physical Review Letters 74, 4101 (1995).

[94] K. Wódkiewicz, Physics Letters A 129, 415 (1988).

[95] C. Miquel, J. P. Paz, and M. Saraceno, Phys Rev A 65, 062309 (2002).

[96] C. Miquel, J. P. Paz, M. Saraceno, E. Knill, R. Laflamme, and C. Negrevergne, Nature 418, 59 (2002).

[97] K. S. Gibbons, M. J. Hoffman, and W. K. Wootters, Phys Rev A 70, 062101 (2004).

[98] K. F. Lee, J. Chen, C. Liang, X. Li, P. L. Voss, and P. Kumar, Opt. Lett. 31, 1905 (2006).

[99] J. Brendel, N. Gisin, W. Tittel, and H. Zbinden, Physical Review Letters 82, 2594 (1999).

[100] A. Poppe et al., Opt Express 12, 3865 (2004).

[101] C. Liang, K. F. Lee, M. Medic, P. Kumar, R. H. Hadfield, and S. W. Nam, Opt Express 15, 1322 (2007).

[102] N. Korolkova, G. Leuchs, R. Loudon, T. C. Ralph, and C. Silberhorn, Phys Rev A 65 (2002).

[103] C. Silberhorn, T. C. Ralph, N. Lutkenhaus, and G. Leuchs, Physical Review Letters 89 (2002).

[104] E. Corndorf, G. Barbosa, C. Liang, H. P. Yuen, and P. Kumar, Opt. Lett. 28, 2040 (2003).

[105] F. Grosshans and P. Grangier, Physical Review Letters 88 (2002).

[106] K. Wen, K. Tamaki, and Y. Yamamoto, Physical Review Letters 103, 170503 (2009).

[107] Y. Zhao, B. Qi, X. Ma, H.-K. Lo, and L. Qian, Physical Review Letters 96, 070502 (2006).

[108] G. A. Barbosa, E. Corndorf, P. Kumar, and H. P. Yuen, Physical Review Letters 90 (2003).

[109] K. F. Lee, Opt. Lett. 34, 1099 (2009).

[110] R. Noe et al., Lightwave Technology, Journal of 17, 1602 (1999). 
[111] J. R. Barry and J. M. Kahn, J Lightwave Technol 10, 1939 (1992).

[112] L. G. Kazovsky, J Lightwave Technol 4, 182 (1986).

[113] P. R. Hemmer, A. Muthukrishnan, M. O. Scully, and M. S. Zubairy, Physical Review Letters 96, 163603 (2006).

[114] S. Buckley, K. Rivoire, and J. Vuckovic, Reports on Progress in Physics 75 (2012).

[115] P. Michler, A. Imamoglu, M. D. Mason, P. J. Carson, G. F. Strouse, and S. K. Buratto, Nature 406, 968 (2000).

[116] A. Ugur, S. Kremling, F. Hatami, S. Hofling, L. Worschech, A. Forchel, and W. T. Masselink, Appl Phys Lett 100 (2012).

[117] N. Mizuochi et al., Nat Photonics 6, 299 (2012).

[118] A. Martin, A. Issautier, H. Herrmann, W. Sohler, D. B. Ostrowsky, O. Alibart, and S. Tanzilli, New Journal of Physics 12 (2010).

[119] H. Takesue, H. Fukuda, T. Tsuchizawa, T. Watanabe, K. Yamada, Y. Tokura, and S.-i. Itabashi, Opt Express 16, 5721 (2008).

[120] X. Y. Li, P. L. Voss, J. E. Sharping, and P. Kumar, Physical Review Letters 94 (2005).

[121] C. Xiong et al., Appl Phys Lett 98 (2011).

[122] S. J. Freedman and J. F. Clauser, Physical Review Letters 28, 938 (1972).

[123] P. Grangier, G. Roger, and A. Aspect, Europhys Lett 1, 173 (1986).

[124] M. Scholz, L. Koch, R. Ullmann, and O. Benson, Appl Phys Lett 94 (2009).

[125] J. Fan, M. D. Eisaman, and A. Migdall, Phys Rev A 76, 043836 (2007).

[126] G. P. Agrawal, Nonlinear fiber optics (Academic Press, San Diego, 1995), 2nd edn., Optics and photonics.

[127] K. Hagimoto and A. Mito, Appl Optics 34, 8276 (1995).

[128] G. P. Agrawal, Applications of nonlinear fiber optics (Academic Press, San Diego, 2001), Optics and photonics.

[129] Q. Lin, F. Yaman, and G. P. Agrawal, Phys Rev A 75 (2007). 
[130] X. Li, P. Voss, J. Chen, K. Lee, and P. Kumar, Opt Express 13, 2236 (2005).

[131] H. Takesue and K. Inoue, Opt Express 13, 7832 (2005).

[132] S. A. E. Lewis, S. V. Chernikov, and J. R. Taylor, Opt. Lett. 24, 1823 (1999).

[133] M. Hirano, T. Nakanishi, T. Okuno, and M. Onishi, Ieee J Sel Top Quant 15, 103 (2009).

[134] J. Chen, K. F. Lee, C. Liang, and P. Kumar, Opt. Lett. 31, 2798 (2006).

[135] E. Y. Zhu et al., Physical Review Letters 108, 213902 (2012).

[136] L. Mandel and E. Wolf, Optical coherence and quantum optics (Cambridge University Press, Cambridge ; New York, 1995).

[137] J.-W. Pan, Z.-B. Chen, C.-Y. Lu, H. Weinfurter, A. Zeilinger, and M. Żukowski, Reviews of Modern Physics 84, 777 (2012).

[138] A. L. Migdall, D. Branning, and S. Castelletto, Phys Rev A 66, 053805 (2002).

[139] D. Y. Vasylyev, A. A. Semenov, and W. Vogel, Physical Review Letters 108, 220501 (2012).

[140] A. A. Semenov, F. Töppel, D. Y. Vasylyev, H. V. Gomonay, and W. Vogel, Phys Rev A 85, 013826 (2012).

[141] A. A. Semenov and W. Vogel, Phys Rev A 81, 023835 (2010).

[142] J. Volz, C. Kurtsiefer, and H. Weinfurter, Appl Phys Lett 79, 869 (2001).

[143] R. Guzzi and R. Rizzi, Appl Optics 23, 1853 (1984).

[144] M. S. R. Subrahmanyam, H. S. Vedanayagam, and P. Venkatacharyulu, J Am Oil Chem Soc 71, 901 (1994).

[145] T. L. Pugh and W. Heller, Journal of Colloid Science 12, 173 (1957).

[146] B. Han, T. Su, H. Wu, Z. Gou, X.-H. Xing, H. Jiang, Y. Chen, X. Li, and J. C. Murrell, Appl Microbiol Biotechnol 83, 669 (2009).

[147] D. Bicout, C. Brosseau, A. S. Martinez, and J. M. Schmitt, Physical Review E 49, 1767 (1994).

[148] A. Einstein, R. Fürth, and A. D. Cowper, Investigations on the theory of the Brownian movement (Methuen \& Co. ltd., London,, 1926). 
[149] E. Waks, K. Inoue, C. Santori, D. Fattal, J. Vuckovic, G. S. Solomon, and Y. Yamamoto, 2004), pp. 76.

[150] B. J. Smith, D. Kundys, N. Thomas-Peter, P. G. R. Smith, and I. A. Walmsley, Opt Express 17, 13516 (2009).

[151] K. F. Reim, P. Michelberger, K. C. Lee, J. Nunn, N. K. Langford, and I. A. Walmsley, Physical Review Letters 107 (2011). 


\section{Appendix A}

\section{Fourier Transform}

For two spatially separated TEMoo beam with a distance about $2 a$ between them, the wave function can be written as

$$
\psi(x) \propto \exp \left[-\frac{(x+a)^{2}}{2 \sigma_{a}^{2}}\right]+\exp \left[-\frac{(x-a)^{2}}{2 \sigma_{a}^{2}}\right]
$$

Given that spatial wave function $\psi(x)$ propagates through a lens with focal length $f$, the $\psi(x)$ in spatial domain can be transformed in to spatial frequency domain by,

$$
\psi(p) \propto \int \exp (i p x) \psi(x) d x
$$

Substituting the $\psi(x)$ in Eq. (A.1) into Eq. (A.2), the wave function can be expressed in spatial frequency domain as,

$$
\psi(p) \propto \int \exp (i p x)\left\{\exp \left[-\frac{(x+a)^{2}}{2 \sigma_{a}^{2}}\right]+\exp \left[-\frac{(x-a)^{2}}{2 \sigma_{a}^{2}}\right]\right\} d x
$$

By using $\exp (i p x)=\cos (p x)+i \sin (p x)$ and given that the integration involving the term $i \sin (p x)$ amounts to zero, we can rewrite the above equation as,

$$
\psi(p) \propto \int \cos (p x)\left\{\exp \left[-\frac{(x+a)^{2}}{2 \sigma_{a}^{2}}\right]\right\} d x+\int \cos (p x)\left\{\exp \left[-\frac{(x-a)^{2}}{2 \sigma_{a}^{2}}\right]\right\} d x
$$


By using the variables transformation, $x^{\prime}=x+a$ and $x^{\prime}=x-a$ for the first and second term in Eq. (A.4) respectively, we rewrite the equation as

$$
\begin{aligned}
\psi(p) & \propto \int \cos \left(p x^{\prime}-p a\right)\left\{\exp \left[-\frac{x^{\prime 2}}{2 \sigma_{a}^{2}}\right]\right\} d x^{\prime} \\
& +\int \cos \left(p x^{\prime}-(-p a)\right)\left\{\exp \left[-\frac{x^{\prime 2}}{2 \sigma_{a}^{2}}\right]\right\} d x^{\prime} .
\end{aligned}
$$

By applying the following trigonometry identities

$$
\begin{gathered}
\cos \left(p x^{\prime}-p a\right)=\cos \left(p x^{\prime}\right) \cos (p a)+\sin \left(p x^{\prime}\right) \sin (p a), \\
\cos \left(p x^{\prime}-(-p a)\right)=\cos \left(p x^{\prime}\right) \cos (-p a)+\sin \left(p x^{\prime}\right) \sin (-p a), \\
\cos (-p a)=\cos (p a),
\end{gathered}
$$

and again considering integrations involving the term $\sin \left(p x^{\prime}\right)$ yield to zero, the equation Eq. (A.4) can be expressed as,

$$
\begin{aligned}
\psi(p) \propto & \cos (p a) \int \cos \left(p x^{\prime}\right)\left\{\exp \left[-\frac{x^{\prime 2}}{2 \sigma_{a}^{2}}\right]\right\} d x^{\prime} \\
& +\cos (p a) \int \cos \left(p x^{\prime}\right)\left\{\exp \left[-\frac{x^{\prime 2}}{2 \sigma_{a}^{2}}\right]\right\} d x^{\prime}
\end{aligned}
$$

Finally by integrating over the $x^{\prime}$, we obtain

$$
\psi(p) \propto \exp \left[-\frac{p^{2} \sigma_{a}^{2}}{2}\right] \cos (p a) .
$$

By substituting 


$$
p=\frac{k x}{f}, \quad D_{p}=\frac{k a}{f}, \quad \sigma_{b}^{2}=\frac{f^{2}}{2 k^{2} \sigma_{a}^{2}}, \text { where } k=\frac{2 \pi}{\lambda}
$$

into Eq. (A.10), We can express $\psi(p)$ in spatial coordinate $x$ as,

$$
\psi(x) \propto \exp \left[-\frac{x^{2}}{2 \sigma_{b}^{2}}\right] \cos \left(x D_{p}\right)
$$




\section{Appendix B}

\section{Relation of Compass States and Beat signal}

To enhance the probability of measuring spatial compass states at center of the chessboard of propensity, we use an imaging system $\left(L_{1}, L_{2}\right)$ for collecting the whole spatial compass states $\odot_{1,2}$ into a single-photon detector. Then, we can selectively projecting the position and momentum of the center spot into the detector. The detector measures the convolution of these spatial compass states as a function of the

relative displacement $d_{x}$, and momentum or tilt $\delta p=\frac{k d_{p}}{f}$; where $d_{p}$ is the displacement associated with the tilt of the lenses $M_{1}$ and $M_{2}$ as depicted in Fig. B.1.

The interference signal is directly proportional to the convolution (overlap) of Wigner distribution for spatial compass states $\bigodot_{1,2}$ at the input lens $L_{1}, L_{2}$ of imaging system. The amplitude of $V_{B}$ can be determined by the spatial overlap of the $\odot_{1,2}$ at the detector plane $Z=z_{D}$, which is given as,

$$
V_{B} \propto \int d x^{\prime} \bigodot_{2}\left(x^{\prime}, z_{D}\right) \bigodot_{1}^{*}\left(x^{\prime}, z_{D}\right)
$$

Where $x^{\prime}$ is the transverse position in detector plane. Translating the $M_{2}$ by a distance $d_{x}$, the compass state has shifted accordingly to give,

$$
V_{B} \propto \int d x^{\prime} \odot_{2}^{*}\left(x^{\prime}-d_{x}, z_{D}\right) \bigodot_{1}\left(x^{\prime}, z_{D}\right)
$$




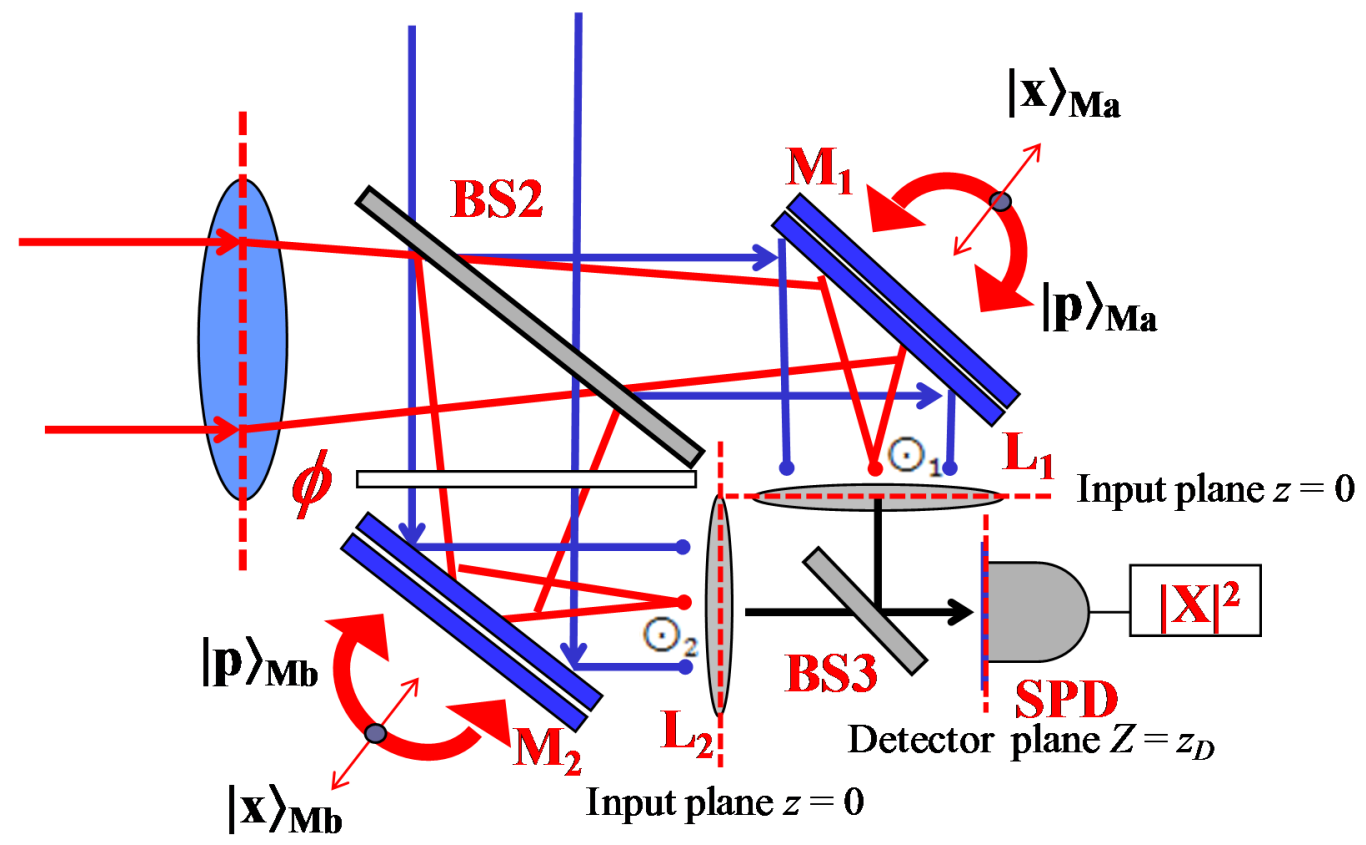

Fig. B.1 The proposed experimental setup for measuring the propensity of two spatial compass states and spatial properties of two mirrors. ( $\mathrm{SMF}=$ single mode fiber, $\mathrm{BS}=$ Beam splitter, $\mathrm{L}=$ lense, $\mathrm{M}=$ mirror, $\mathrm{SPD}=$ single photon detector)

As shown in Fig. B.1, each spatial compass state passes through the lenses $\left(L_{1}, L_{2}\right)$ and picks up the quadrature-phase term $\exp \left[-i \frac{k x^{2}}{2 f}\right]$. From paraxial approximation of the compass states $\bigodot_{1,2}$ at the input plane $Z=0$ after the lenses can be described as,

$$
\begin{gathered}
\bigodot_{1\left(L_{1}\right)}(x, Z=0)=\exp \left[-i \frac{k x^{2}}{2 f}\right] \bigodot_{1}(x, Z=0), \\
\bigodot_{2\left(L_{2}\right)}\left(x-d_{x}, Z=0\right)=\exp \left[-i \frac{k x^{2}}{2 f}\right] \bigodot_{2}\left(x-d_{x}, Z=0\right) .
\end{gathered}
$$

When the lens $L_{1}$ is scanned by a distance $d_{p}$, the spatially varying phase acquired by the $\bigodot_{1\left(L_{1}\right)}$ shifted and expression for $\bigodot_{1\left(L_{1}\right)}$ in $(\mathrm{B} .3)$ is given as, 


$$
\bigodot_{1\left(L_{1}\right)}(x, Z=0)=\exp \left[-i \frac{k}{2 f}\left(x-d_{p}\right)^{2}\right] \bigodot_{1}(x, Z=0) .
$$

After the lenses, each compass state $\odot_{1,2}$ propagates a distance of $f$ to reach the single photon detector. The compass state $\bigodot_{1,2}$ at detector plane $Z=z_{D}$, can be obtained using Fresnel's diffraction integral as,

$$
\begin{aligned}
\odot_{1}\left(x^{\prime}, z_{D}\right) & =\sqrt{\frac{k}{i 2 \pi f}} \int d x \exp \left[i \frac{k}{2 f}\left(x-x^{\prime}\right)^{2}\right] \\
& \times \exp \left[-i \frac{k}{2 f}\left(x-d_{p}\right)^{2}\right] \odot_{1}(x, Z=0), \\
\bigodot_{2}\left(x^{\prime}-d_{x}, z_{D}\right) & =\sqrt{\frac{k}{i 2 \pi f}} \int d x \exp \left[i \frac{k}{2 f}\left(x-x^{\prime}\right)^{2}\right] \\
& \times \exp \left[-i \frac{k}{2 f} x^{2}\right] \odot_{2}\left(x-d_{x}, Z=0\right),
\end{aligned}
$$

As detector plane is coincides with the focal planes of the lenses $\left(L_{1}, L_{2}\right)$, the quadratic phases involving $x^{2}$ cancel in these equations above and simplified as,

$$
\begin{aligned}
\odot_{1}\left(x^{\prime}, z_{D}\right) & =\sqrt{\frac{k}{i 2 \pi f}} \int d x \exp \left[i \frac{k}{2 f}\left(x^{\prime 2}-d_{p}{ }^{2}\right)\right] \\
& \times \exp \left[-i \frac{k}{f} x\left(x^{\prime}-d_{p}\right)\right] \odot_{1}(x, Z=0), \\
\bigodot_{2}\left(x^{\prime}-d_{x}, z_{D}\right) & =\sqrt{\frac{k}{i 2 \pi f}} \int d x \exp \left[i \frac{k}{2 f} x^{\prime 2}\right] \\
& \times \exp \left[-i \frac{k}{f} x x^{\prime}\right] \odot_{2}\left(x-d_{x}, Z=0\right),
\end{aligned}
$$


By substituting the equations above into Eq. (B.2), quadratic phases involving $x^{\prime 2}$ vanish and we obtain the interference amplitude as

$$
\begin{gathered}
V_{B}\left(d_{x}, d_{p}\right)=\frac{k}{2 \pi f} \exp \left(-i \frac{\mathrm{k}}{2 f} d_{p}^{2}\right) \int d x^{\prime} \int d x_{1} \exp \left[-i \frac{k}{f} x x^{\prime}\right] \odot_{2}\left(x_{1}-d_{x}, Z=0\right) \\
\times \int d x_{2} \exp \left[-i \frac{k}{f} x_{2}\left(x^{\prime}-d_{p}\right)\right] \bigodot_{1}\left(x_{2}, Z=0\right)
\end{gathered}
$$

The integrating over $x^{\prime}$ yields a delta function as

$$
\int d x^{\prime} \exp \left[-i \frac{k}{f} x^{\prime}\left(x_{1}-x_{2}\right)\right]=2 \pi \delta\left(x_{1}-x_{2}\right)
$$

$x_{1}$ and $x_{2}$ are dummy variables for integration involving the compass states $\bigodot_{2}$ and $\bigodot_{1}$. Then, we rewrite the Eq.(B.10) as

$$
\begin{aligned}
V_{B}\left(d_{x}, d_{p}\right) & =\frac{k}{f} \exp \left(-i \frac{\mathrm{k}}{2 f} d_{p}^{2}\right) \int d x_{2} \exp \left[-i \frac{k}{f} x_{2} d_{p}\right] \bigodot_{1}\left(x_{2}, Z=0\right) \\
& \times \int d x_{1} \odot_{2}\left(x_{1}-d_{x}, Z=0\right) \delta\left(x_{1}-x_{2}\right) .
\end{aligned}
$$

Now, integrating over $x_{1}$, the Eq.(B.12) becomes,

$$
V_{B}\left(d_{x}, d_{p}\right)=\frac{k}{f} \exp \left(-i \frac{\mathrm{k}}{2 f} d_{p}^{2}\right) \int d x_{2} \exp \left[-i \frac{k}{f} x_{2} d_{p}\right] \bigodot_{2}\left(x_{2}-d_{x}, z=0\right) \odot_{1}\left(x_{2}, Z=0\right)
$$

By changing the variable $x_{2}$ by $x$ and dropping the $Z=0$, the mean square amplitude is given as,

$$
\left|V_{B}\left(d_{x}, d_{p}\right)\right|^{2} \propto\left|\int d x \bigodot_{2}\left(x-d_{x}\right) \bigodot_{1}(x) \exp \left[-i \frac{k d_{p}}{f} x\right]\right|^{2}
$$


We can rewrite the above equation as,

$$
\begin{aligned}
\left|V_{B}\left(d_{x}, d_{p}\right)\right|^{2} & \propto \int d x \bigodot_{2}^{*}\left(x-d_{x}\right) \bigodot_{1}(x) \exp \left[-i \frac{k d_{p}}{f} x\right] \\
& \times \int d x^{\prime} \bigodot_{2}\left(x^{\prime}-d_{x}\right) \bigodot_{1}^{*}\left(x^{\prime}\right) \exp \left[i \frac{k d_{p}}{f} x^{\prime}\right] .
\end{aligned}
$$

By using the variables transformation,

$$
\begin{aligned}
& x=x_{o}+\frac{\eta}{2} \\
& x^{\prime}=x_{o}-\frac{\eta}{2}
\end{aligned}
$$

and since the Jacobian of this transformation is 1 . The Eq.(B.15) can be written in term of these variables as,

$$
\begin{gathered}
\left|V_{B}\left(d_{x}, d_{p}\right)\right|^{2} \propto \int d x_{o} \int d \eta \bigodot_{2}^{*}\left(x_{o}+\frac{\eta}{2}-d_{x}\right) \bigodot_{2}\left(x_{o}-\frac{\eta}{2}-d_{x}\right) \\
\times \bigodot_{1}\left(x_{o}+\frac{\eta}{2}\right) \bigodot_{1}^{*}\left(x_{o}-\frac{\eta}{2}\right) \exp \left[-i \frac{k d_{p}}{f} \eta\right] .
\end{gathered}
$$

From the definition of the Wigner function,

$$
\mathcal{W}(x, p)=\frac{1}{2 \pi} \int_{-\infty}^{\infty} d \varepsilon e^{-i \varepsilon p} \psi^{*}\left(x+\frac{\varepsilon}{2}\right) \psi\left(x-\frac{\varepsilon}{2}\right)
$$

where its inverse transform is given by,

$$
\psi^{*}\left(x+\frac{\varepsilon}{2}\right) \psi\left(x-\frac{\varepsilon}{2}\right)=\int_{-\infty}^{\infty} d p e^{-i \varepsilon p} \mathcal{W}(x, p)
$$

Then, we can write 


$$
\bigodot_{1}\left(x_{o}+\frac{\eta}{2}\right) \bigodot_{1}^{*}\left(x_{o}-\frac{\eta}{2}\right)=\int_{-\infty}^{\infty} d p e^{-i \eta p} \mathcal{W}(x, p)_{\odot_{1}}
$$

and obtain Eq. (B.16) as

$$
\begin{aligned}
\left|V_{B}\left(d_{x}, d_{p}\right)\right|^{2} \propto \int & d x_{o} \int d \eta \odot_{2}^{*}\left(x_{o}+\frac{\eta}{2}-d_{x}\right) \odot_{2}\left(x_{o}-\frac{\eta}{2}-d_{x}\right) \\
& \times \int d \exp \left[-i \frac{k d_{p}}{f} \eta\right] \exp [-i \eta p] \mathcal{W}(x, p)_{\odot_{1}}
\end{aligned}
$$

Again, by the definition of the Wigner function we can write,

$$
\begin{aligned}
\mathcal{W}\left(x-d_{x}, p+\frac{k d_{p}}{f}\right)_{\odot_{2}} & =\int \frac{d \eta}{2 \pi} \exp \left[-i\left(p+\frac{k d_{p}}{f}\right) \eta\right] \\
& \times \odot_{2}^{*}\left(x_{o}+\frac{\eta}{2}-d_{x}\right) \odot_{2}\left(x_{o}-\frac{\eta}{2}-d_{x}\right) .
\end{aligned}
$$

Substituting Eq.(B.20) in Eq.(B.19), the mean square interference signal amplitude can be expressed as

$$
\left|V_{B}\left(d_{x}, d_{p}\right)\right|^{2} \propto \int d x d p \mathcal{W}\left(x-d_{x}, p+\frac{k d_{p}}{f}\right)_{\odot_{2}} \mathcal{W}(x, p)_{\odot_{1}}
$$

Where $\mathcal{W}(x, p)_{\odot_{1}}$ is the Wigner distribution of the compass states $\odot_{1}$ in the input plane of the $L_{1}$ and $\mathcal{W}\left(x-d_{x}, p+\frac{k d_{p}}{f}\right)_{\odot_{2}}$ is the Wigner distribution of the compass states $\odot_{2}$ in the input plane of the $L_{2}$. 


\section{Appendix C}

\section{Wigner distribution of Spatial Compass state}

The spatial compass states $\bigodot_{1}$ and $\odot_{2}$ can be expressed in position coordinates in a identical form as

$$
\begin{aligned}
\odot_{1,2} & \propto \psi_{1,2}^{t}(x)+\psi_{1,2}^{r}(x) \\
& \propto \exp \left[-\frac{(x+a)^{2}}{2 \sigma_{a}^{2}}\right]+\exp \left[-\frac{(x-a)^{2}}{2 \sigma_{a}^{2}}\right]+\exp \left[\frac{x^{2}}{2 \sigma_{b}^{2}}\right] \cos \left(x D_{p}\right) .
\end{aligned}
$$

Where the first and second terms are corresponding to $\psi_{1,2}^{t}(x)$; the third term is corresponding to $\psi_{1,2}^{r}(x)$. According to the definition given in Eq. 3.1, the Wigner function of the spatial compass states $\bigodot_{1}$ is given as

$$
\mathcal{W}(x, p)_{1} \propto \int_{-\infty}^{\infty} d \varepsilon e^{-i \varepsilon p} \bigodot_{1}{ }^{*}\left(x+\frac{\varepsilon}{2}\right) \bigodot_{1}\left(x-\frac{\varepsilon}{2}\right)
$$

By substituting $\bigodot_{1}$ in Eq. (C.1) to Eq. (C.3), we obtain the Wigner function as,

$$
\begin{aligned}
\mathcal{W}(x, p)_{1} \propto & \int_{-\infty}^{\infty} d \varepsilon e^{-i \varepsilon p} \psi_{1}^{* t}\left(x+\frac{\varepsilon}{2}\right) \psi_{1}^{t}\left(x-\frac{\varepsilon}{2}\right) \\
& +\int_{-\infty}^{\infty} d \varepsilon e^{-i \varepsilon p} \psi_{1}^{* r}\left(x+\frac{\varepsilon}{2}\right) \psi_{1}^{r}\left(x-\frac{\varepsilon}{2}\right)
\end{aligned}
$$




$$
\begin{aligned}
& +\int_{-\infty}^{\infty} d \varepsilon e^{-i \varepsilon p} \psi_{1}^{* t}\left(x+\frac{\varepsilon}{2}\right) \psi_{1}^{r}\left(x-\frac{\varepsilon}{2}\right) \\
& +\int_{-\infty}^{\infty} d \varepsilon e^{-i \varepsilon p} \psi_{1}^{* r}\left(x+\frac{\varepsilon}{2}\right) \psi_{1}^{t}\left(x-\frac{\varepsilon}{2}\right) .
\end{aligned}
$$

\section{Chessboard pattern}

The chessboard pattern of the Wigner function is contributed from the components $\psi_{1}^{* t}\left(x+\frac{\varepsilon}{2}\right) \psi_{1}^{t}\left(x-\frac{\varepsilon}{2}\right)$ and $\psi_{1}^{* r}\left(x+\frac{\varepsilon}{2}\right) \psi_{1}^{r}\left(x-\frac{\varepsilon}{2}\right)$ in Eq. (C.4) and Eq.

(C.5). The Wigner function of the first component is given by

$$
\mathcal{W}(x, p)_{t t} \propto \int_{-\infty}^{\infty} \psi_{1}^{t *}\left(x+\frac{\varepsilon}{2}\right) \psi_{1}^{t}\left(x-\frac{\varepsilon}{2}\right) d \varepsilon e^{-i \varepsilon p}
$$

From Eq. (C.2), we can rewrite the above equation as

$$
\begin{aligned}
\mathcal{W}(x, p)_{t t} \propto \int_{-\infty}^{\infty}\left(\exp \left[-\frac{\left(x+a+\frac{\varepsilon}{2}\right)^{2}}{2 \sigma_{a}^{2}}\right]+\exp \left[-\frac{\left(x-a+\frac{\varepsilon}{2}\right)^{2}}{2 \sigma_{a}^{2}}\right]\right) \\
\quad\left(\exp \left[-\frac{\left(x+a+\frac{\varepsilon}{2}\right)^{2}}{2 \sigma_{a}^{2}}\right]+\exp \left[-\frac{\left(x-a+\frac{\varepsilon}{2}\right)^{2}}{2 \sigma_{a}^{2}}\right]\right) d \varepsilon e^{-i \varepsilon p}, \\
\propto \int_{-\infty}^{\infty}\left(\exp \left[-\frac{2(x+a)^{2}+\frac{\varepsilon^{2}}{2}}{2 \sigma_{a}^{2}}\right]+\exp \left[-\frac{2 x^{2}+2\left(a+\frac{\varepsilon}{2}\right)^{2}}{2 \sigma_{a}^{2}}\right]\right. \\
\left.+\exp \left[-\frac{2 x^{2}+2\left(a-\frac{\varepsilon}{2}\right)^{2}}{2 \sigma_{a}^{2}}\right]+\exp \left[-\frac{2(x-a)^{2}+\frac{\varepsilon^{2}}{2}}{2 \sigma_{a}^{2}}\right]\right) d \varepsilon e^{-i \varepsilon p}
\end{aligned}
$$

Integrating the first term in Eq. (C.10), we obtain 


$$
\int_{-\infty}^{\infty} \exp \left[-\frac{2(x+a)^{2}+\frac{\varepsilon^{2}}{2}}{2 \sigma_{a}^{2}}\right] d \varepsilon e^{-i \varepsilon p}=\exp \left[-\frac{2(x+a)^{2}}{2 \sigma_{a}^{2}}\right] \int_{-\infty}^{\infty} \exp \left[-\frac{\frac{\varepsilon^{2}}{2}}{2 \sigma_{a}^{2}}\right] d \varepsilon e^{-i \varepsilon p}
$$

By using $\exp (i \varepsilon p)=\cos (\varepsilon p)+i \sin (\varepsilon p)$ and the integration involving the term $i \sin (\varepsilon p)$ amounts to zero, we can rewrite the above equation as,

$$
\begin{aligned}
\int_{-\infty}^{\infty} \exp \left[-\frac{2(x+a)^{2}+\frac{\varepsilon^{2}}{2}}{2 \sigma_{a}^{2}}\right] d \varepsilon e^{-i \varepsilon p} & =\exp \left[-\frac{2(x+a)^{2}}{2 \sigma_{a}^{2}}\right] \int_{-\infty}^{\infty} \exp \left[-\frac{\varepsilon^{2}}{2 \sigma_{a}^{2}}\right](\cos (\varepsilon p)) d \varepsilon \\
& \propto \exp \left[-\frac{2(x+a)^{2}}{2 \sigma_{a}^{2}}\right] \exp \left[-p^{2} \sigma_{a}^{2}\right]
\end{aligned}
$$

By similar fashion, we can obtain the Wigner function for the fourth term in Eq.

(C.10) as

$$
\int_{-\infty}^{\infty} \exp \left[-\frac{2(x-a)^{2}+\frac{\varepsilon^{2}}{2}}{2 \sigma_{a}^{2}}\right] d \varepsilon e^{-i \varepsilon p} \propto \exp \left[-\frac{2(x-a)^{2}}{2 \sigma_{a}^{2}}\right] \exp \left[-p^{2} \sigma_{a}^{2}\right]
$$

On the other hand, the Wigner function for the second term in Eq. (C.10) is given as

$$
\begin{aligned}
\int_{-\infty}^{\infty} \exp \left[-\frac{2 x^{2}+2\left(a+\frac{\varepsilon}{2}\right)^{2}}{2 \sigma_{a}^{2}}\right] d \varepsilon e^{-i \varepsilon p} & =\exp \left[-\frac{x^{2}}{\sigma_{a}^{2}}\right] \int_{-\infty}^{\infty} \exp \left[-\frac{\left(a-\frac{\varepsilon}{2}\right)^{2}}{\sigma_{a}^{2}}\right] d \varepsilon e^{-i \varepsilon p} \\
& =\exp \left[-\frac{x^{2}}{\sigma_{a}^{2}}\right] \int_{-\infty}^{\infty} \exp \left[-\frac{\left(a-\frac{\varepsilon}{2}\right)^{2}}{\sigma_{a}^{2}}\right](\cos (\varepsilon p)) d \varepsilon
\end{aligned}
$$

By using the variables transformation,

$$
\frac{\varepsilon^{\prime}}{2}=a-\frac{\varepsilon}{2}
$$

The Eq.(C.13) can be written in term of $\varepsilon^{\prime}$ as, 


$$
\propto \exp \left[-\frac{x^{2}}{\sigma_{a}^{2}}\right] \int_{-\infty}^{\infty} \exp \left[-\frac{\left(\varepsilon^{\prime} / 2\right)^{2}}{\sigma_{a}^{2}}\right]\left(\cos \left(p \varepsilon^{\prime}-2 p a\right)\right) d \varepsilon
$$

By applying the following trigonometry identities

$$
\begin{gathered}
\cos \left(p \varepsilon^{\prime}-2 p a\right)=\cos \left(p \varepsilon^{\prime}\right) \cos (-p a)+\sin \left(p \varepsilon^{\prime}\right) \sin (-p a), \\
\cos (-p a)=\cos (p a),
\end{gathered}
$$

and considering integration involving the term $\sin \left(p \varepsilon^{\prime}\right)$ yield to zero, the Eq. (C.14) can be expressed as,

$$
\begin{aligned}
& \propto \exp \left[-\frac{x^{2}}{\sigma_{a}^{2}}\right] \cos (2 p a) \int_{-\infty}^{\infty} \exp \left[-\frac{\left(\varepsilon^{\prime} / 2\right)^{2}}{\sigma_{a}^{2}}\right]\left(\cos p \varepsilon^{\prime}\right) d \varepsilon, \\
& \propto \exp \left[-\frac{x^{2}}{\sigma_{a}^{2}}\right] \exp \left[-p^{2} \sigma_{a}^{2}\right] \cos (2 p a),
\end{aligned}
$$

Similarly, we can obtain the Wigner function for the 3rd term in Eq. (C.10) as

$$
\propto \exp \left[-\frac{x^{2}}{\sigma_{a}^{2}}\right] \exp \left[-p^{2} \sigma_{a}^{2}\right] \cos (2 p a)
$$

Finally the summation of Wigner functions for all four terms in Eq. (C.10) yields,

$$
\begin{aligned}
\mathcal{W}(x, p)_{t t} \propto & \exp \left[-\frac{(x+a)^{2}}{\sigma_{a}^{2}}-p^{2} \sigma_{a}^{2}\right]+\exp \left[-\frac{(x-a)^{2}}{\sigma_{a}^{2}}-p^{2} \sigma_{a}^{2}\right] \\
& +2 \exp \left[-\frac{x^{2}}{\sigma_{a}^{2}}-p^{2} \sigma_{a}^{2}\right] \cos (2 p a) .
\end{aligned}
$$

The Wigner function of the $\psi_{1}^{* r}\left(x+\frac{\varepsilon}{2}\right) \psi_{1}^{r}\left(x-\frac{\varepsilon}{2}\right)$ is given by 


$$
\mathcal{W}(x, p)_{r r} \propto \int_{-\infty}^{\infty} \psi_{1}^{r *}\left(x+\frac{\varepsilon}{2}\right) \psi_{1}^{r}\left(x-\frac{\varepsilon}{2}\right) d \varepsilon e^{-i \varepsilon p}
$$

From Eq. (C.2), we can rewrite the Eq. (C.20) as

$$
\begin{aligned}
\mathcal{W}(x, p)_{r r} \propto \int_{-\infty}^{\infty}\left[\exp \left[\frac{\left(x+\frac{\varepsilon}{2}\right)^{2}}{2 \sigma_{b}^{2}}\right] \cos \left(\left(x+\frac{\varepsilon}{2}\right) D_{p}\right)\right] \\
{\left[\exp \left[\frac{\left(x-\frac{\varepsilon}{2}\right)^{2}}{2 \sigma_{b}^{2}}\right] \cos \left(\left(x-\frac{\varepsilon}{2}\right) D_{p}\right)\right] d \varepsilon e^{-i \varepsilon p}, } \\
\propto \int_{-\infty}^{\infty}\left[\exp \left[\frac{\left(x+\frac{\varepsilon}{2}\right)^{2}}{2 \sigma_{b}^{2}}\right] \exp \left[\frac{\left(x-\frac{\varepsilon}{2}\right)^{2}}{2 \sigma_{b}^{2}}\right]\right] \\
{\left[\cos \left(\left(x+\frac{\varepsilon}{2}\right) D_{p}\right) \cos \left(\left(x-\frac{\varepsilon}{2}\right) D_{p}\right)\right] d \varepsilon e^{-i \varepsilon p} . }
\end{aligned}
$$

By simplifying the exponential terms and applying the trigonometric identity

$$
\begin{aligned}
\cos \left(\left(x+\frac{\varepsilon}{2}\right) D_{p}\right) \cos \left(\left(x-\frac{\varepsilon}{2}\right) D_{p}\right)= & \frac{1}{2}\left\{\cos \left(\left(x+\frac{\varepsilon}{2}\right) D_{p}-\left(x-\frac{\varepsilon}{2}\right) D_{p}\right)\right. \\
& \left.+\cos \left(\left(x+\frac{\varepsilon}{2}\right) D_{p}+\left(x-\frac{\varepsilon}{2}\right) D_{p}\right)\right\}
\end{aligned}
$$

we can rewrite Eq. (C.20) as

$$
\begin{aligned}
& \propto \exp \left[-\frac{x^{2}}{\sigma_{b}^{2}}\right] \cos \left(x D_{p}\right) \int_{-\infty}^{\infty} \exp \left[-\frac{\varepsilon^{2}}{4 \sigma_{b}^{2}}\right] d \varepsilon e^{-i \varepsilon p} \\
& +\exp \left[-\frac{x^{2}}{\sigma_{b}^{2}}\right] \int_{-\infty}^{\infty} \cos \left(\varepsilon D_{p}\right) \exp \left[-\frac{\varepsilon^{2}}{4 \sigma_{b}^{2}}\right] d \varepsilon e^{-i \varepsilon p}
\end{aligned}
$$


By using $e^{-i \varepsilon p}=\cos (\varepsilon p)+i \sin (\varepsilon p)$ and the integration involving the term $i \sin (\varepsilon p)$ amounts to zero, we can rewrite the above equation as,

$$
\begin{aligned}
& \propto \exp \left[-\frac{x^{2}}{\sigma_{b}^{2}}\right] \cos \left(x D_{p}\right) \int_{-\infty}^{\infty} \exp \left[-\frac{\varepsilon^{2}}{4 \sigma_{b}^{2}}\right] \cos (\varepsilon p) d \varepsilon \\
& +\exp \left[-\frac{x^{2}}{\sigma_{b}^{2}}\right] \int_{-\infty}^{\infty} \exp \left[-\frac{\varepsilon^{2}}{4 \sigma_{b}^{2}}\right] \cos \left(\varepsilon D_{p}\right) \cos (\varepsilon p) d \varepsilon
\end{aligned}
$$

Again, we expand the equation above using trigonometric identity to obtain

$$
\begin{aligned}
& \propto \exp \left[-\frac{x^{2}}{\sigma_{b}^{2}}\right] \cos \left(x D_{p}\right) \int_{-\infty}^{\infty} \exp \left[-\frac{\varepsilon^{2}}{4 \sigma_{b}^{2}}\right] \cos (\varepsilon p) d \varepsilon \\
& +\frac{1}{2} \exp \left[-\frac{x^{2}}{\sigma_{b}^{2}}\right] \int_{-\infty}^{\infty} \cos \left(\varepsilon\left(p+D_{p}\right)\right) \exp \left[-\frac{\varepsilon^{2}}{4 \sigma_{b}^{2}}\right] d \varepsilon \\
& +\frac{1}{2} \exp \left[-\frac{x^{2}}{\sigma_{b}^{2}}\right] \int_{-\infty}^{\infty} \cos \left(\varepsilon\left(p-D_{p}\right)\right) \exp \left[-\frac{\varepsilon^{2}}{4 \sigma_{b}^{2}}\right] d \varepsilon
\end{aligned}
$$

Integrating over $\varepsilon$ for all three terms in Eq. (C.25), we can obtain

$$
\begin{aligned}
\mathcal{W}(x, p)_{r r} & \propto \exp \left[-\frac{(x)^{2}}{\sigma_{b}^{2}}-\left(p+D_{p}\right)^{2} \sigma_{b}^{2}\right]+\exp \left[-\frac{(x)^{2}}{\sigma_{b}^{2}}-\left(p-D_{p}\right)^{2} \sigma_{b}^{2}\right] \\
& +2 \exp \left[-\frac{(x)^{2}}{\sigma_{b}^{2}}-p^{2} \sigma_{b}^{2}\right] \cos \left(2 x D_{p}\right)
\end{aligned}
$$

The chessboard pattern in the Wigner function arises from the summation of the interference terms $\exp \left[-\frac{x^{2}}{\sigma_{a}^{2}}-p^{2} \sigma_{a}^{2}\right] \cos (2 p a)$ and $\exp \left[-\frac{(x)^{2}}{\sigma_{b}^{2}}-p^{2} \sigma_{b}^{2}\right] \cos \left(2 x D_{p}\right)$ in Eq. (C.19) and Eq. (C.26). 


\section{Appendix D}

\section{Propensity $\mathcal{P}_{b}\left(d_{x}, d_{p}\right)$}

The propensity is the mean-square interference signal that measured after the squarer, $X^{2}$. Direct measurement of the propensity measures the orthogonality of the compass states $\odot_{1}$ and $\odot_{2}$. By using the imaging system, we can project the center of the chessboard for the maximum beat of $\mathcal{P}_{b}\left(d_{x}, d_{p}\right)$ at around $d_{x} \sim 0$ and $d_{p} \sim 0$. The spatial coordinates of the chessboard pattern in the propensity exactly correspond to the coordinate system of the imaging system.

The interference signal as a function of $d_{x}$ and $d_{p}$ is given by

$$
V_{B}\left(d_{x}, d_{p}\right) \propto \frac{k}{f} \exp \left(i \frac{\mathrm{k} d_{p}^{2}}{2 f}\right) \int_{-\infty}^{\infty} d x^{\prime} \exp \left(-i \frac{\mathrm{k} x^{\prime} d_{p}}{f}\right) \odot_{2}{ }^{*}\left(x^{\prime}-d_{x}\right) \odot_{1}\left(x^{\prime}\right)
$$

Where

$$
\begin{aligned}
\odot_{1}\left(x^{\prime}\right) & \propto \exp \left[-\frac{\left(x^{\prime}+a\right)^{2}}{2 \sigma_{a}^{2}}\right]+\exp \left[-\frac{\left(x^{\prime}-a\right)^{2}}{2 \sigma_{a}^{2}}\right]+\exp \left[-\frac{x^{\prime 2}}{2 \sigma_{b}^{2}}\right] \cos \left(x^{\prime} D_{p}\right) \\
& \propto \odot_{1_{\mathbf{I}}}+\odot_{1_{\text {II }}}+\odot_{1_{\text {III }}} \\
\odot_{2}^{*}\left(x^{\prime}-d_{x}\right) & \propto \exp \left[-\frac{\left(x^{\prime}-d_{x}+a\right)^{2}}{2 \sigma_{a}^{2}}\right]+\exp \left[-\frac{\left(x^{\prime}-d_{x}-a\right)^{2}}{2 \sigma_{a}^{2}}\right]
\end{aligned}
$$




$$
\begin{aligned}
& +\exp \left[-\frac{\left(x^{\prime}-d_{x}\right)^{2}}{2 \sigma_{b}^{2}}\right] \cos \left(\left(x^{\prime}-d_{x}\right) D_{p}\right) \\
\propto & \bigodot_{2}{ }^{*}+\bigodot_{2_{\mathbf{I I}}}{ }^{*}+\bigodot_{2_{\mathbf{I I I}}}{ }^{*},
\end{aligned}
$$

As the interference signal $V_{B}\left(d_{x}, d_{p}\right)$ is directly proportional to the spatial overlapping of the compass states $\bigodot_{1}$ and $\bigodot_{2}{ }^{*}$. The product terms in Eq. (D.1) that will contribute to the interference signal $V_{B}\left(d_{x}, d_{p}\right)$ are $\bigodot_{2_{\mathbf{I}}}{ }^{*} \bigodot_{1_{\mathbf{I}}}, \bigodot_{2_{\text {II }}}{ }^{*} \bigodot_{1_{\text {II }}}$, and $\bigodot_{2 \text { III }}{ }^{*} \bigodot_{1_{\text {III }}}$.

The contribution from $\bigodot_{2_{\mathbf{I}}}{ }^{*} \bigodot_{1_{\mathbf{I}}}$ can be expressed as

$$
V_{B_{\mathbf{I}}}\left(d_{x}, d_{p}\right) \propto \int_{-\infty}^{\infty} d x^{\prime} \exp \left(-i \frac{\mathrm{k} x^{\prime} d_{p}}{f}\right) \exp \left[-\frac{\left(x^{\prime}-d_{x}+a\right)^{2}}{2 \sigma_{a}^{2}}\right] \exp \left[-\frac{\left(x^{\prime}+a\right)^{2}}{2 \sigma_{a}^{2}}\right]
$$

By using the variable transformation,

$$
u=x^{\prime}+a,
$$

we rewrite Eq. (D.4) as

$$
\begin{aligned}
& V_{B_{\mathbf{I}}}\left(d_{x}, d_{p}\right) \propto \exp \left(i \frac{a \mathrm{k} d_{p}}{f}\right) \exp \left[-\frac{d_{x}^{2}}{2 \sigma_{a}^{2}}\right] \int_{-\infty}^{\infty} d u \exp \left[-\frac{1}{\sigma_{a}^{2}}\left(u^{2}+u B\right)\right] \\
& \quad \propto \exp \left(i \frac{a \mathrm{k} d_{p}}{f}\right) \exp \left[-\frac{d_{x}^{2}}{2 \sigma_{a}^{2}}\right] \exp \left[-\frac{B^{2} \sigma_{a}^{2}}{4}\right] \int_{-\infty}^{\infty} d u \exp \left[-\frac{1}{\sigma_{a}^{2}}\left(u+\frac{B}{2}\right)^{2}\right] \\
& \propto \sqrt{\sigma_{a}^{2} \pi} \exp \left(i \frac{a \mathrm{k} d_{p}}{f}\right) \exp \left[-\frac{d_{x}^{2}}{2 \sigma_{a}^{2}}\right] \exp \left[-\frac{B^{2} \sigma_{a}^{2}}{4}\right] .
\end{aligned}
$$


where $B=-d_{x}+i \frac{\mathrm{k} d_{p} \sigma_{a}^{2}}{f}$.

Similarly, the contribution from $\bigodot_{2_{\text {II }}}{ }^{*} \bigodot_{1_{\text {II }}}$ is given as

$$
V_{B_{\mathrm{II}}}\left(d_{x}, d_{p}\right) \propto \int_{-\infty}^{\infty} d x^{\prime} \exp \left(-i \frac{\mathrm{k} x^{\prime} d_{p}}{f}\right) \exp \left[-\frac{\left(x^{\prime}-d_{x}-a\right)^{2}}{2 \sigma_{a}^{2}}\right] \exp \left[-\frac{\left(x^{\prime}-a\right)^{2}}{2 \sigma_{a}^{2}}\right]
$$

By using the variables transformation,

$$
u=x^{\prime}-a,
$$

we rewrite Eq. (D.7) as

$$
V_{B_{\mathrm{II}}}\left(d_{x}, d_{p}\right) \propto \exp \left(-i \frac{a \mathrm{k} d_{p}}{f}\right) \exp \left[-\frac{d_{x}^{2}}{2 \sigma_{a}^{2}}\right] \int_{-\infty}^{\infty} d u \exp \left[-\frac{1}{\sigma_{a}^{2}}\left(u^{2}+u B\right)\right]
$$

(D.8)

$$
\begin{aligned}
& \propto \exp \left(-i \frac{a \mathrm{k} d_{p}}{f}\right) \exp \left[-\frac{d_{x}^{2}}{2 \sigma_{a}^{2}}\right] \exp \left[-\frac{B^{2} \sigma_{a}^{2}}{4}\right] \int_{-\infty}^{\infty} d u \exp \left[-\frac{1}{\sigma_{a}^{2}}\left(u+\frac{B}{2}\right)^{2}\right] \\
& \propto \sqrt{\sigma_{a}^{2} \pi} \exp \left(-i \frac{a \mathrm{k} d_{p}}{f}\right) \exp \left[-\frac{d_{x}^{2}}{2 \sigma_{a}^{2}}\right] \exp \left[-\frac{B^{2} \sigma_{a}^{2}}{4}\right] .
\end{aligned}
$$

where $=-d_{x}+i \frac{\mathrm{k} d_{p} \sigma_{a}^{2}}{f}$

Furthermore the contribution from $\bigodot_{2}{ }_{\text {III }}{ }^{*} \bigodot_{1_{\text {III }}}$ is given as

$$
\begin{gathered}
V_{B_{\mathrm{III}}}\left(d_{x}, d_{p}\right) \propto \int_{-\infty}^{\infty} \exp \left(-i \frac{\mathrm{k} x^{\prime} d_{p}}{f}\right) \exp \left[-\frac{\left(x^{\prime}-d_{x}\right)^{2}}{2 \sigma_{b}^{2}}\right] \exp \left[-\frac{x^{\prime 2}}{2 \sigma_{b}^{2}}\right] \\
\cos \left(\left(x^{\prime}-d_{x}\right) D_{p}\right) \cos \left(x^{\prime} D_{p}\right) d x^{\prime}
\end{gathered}
$$




$$
\begin{aligned}
& \propto \exp \left[-\frac{d_{x}^{2}}{2 \sigma_{b}^{2}}\right] \int_{-\infty}^{\infty} \exp \left[-\frac{\left(x^{\prime 2}+V x^{\prime}\right)}{\sigma_{b}^{2}}\right] \\
& \left.\qquad \cos \left(2 x^{\prime} D_{p}-d_{x} D_{p}\right)+\cos \left(-d_{x} D_{p}\right)\right\} d x^{\prime} .
\end{aligned}
$$

Where $V=-d_{x}+i \frac{\mathrm{k} d_{p} \sigma_{b}^{2}}{f}$. Then, we rearrange Eq. (D.11) as

$$
\begin{aligned}
V_{B_{\mathrm{III}}}\left(d_{x}, d_{p}\right) \propto & \exp \left[-\frac{d_{x}^{2}}{2 \sigma_{b}^{2}}\right] \exp \left[\frac{V^{2}}{4 \sigma_{b}^{2}}\right] \int_{-\infty}^{\infty} \exp \left[-\frac{\left(x^{\prime}+\frac{V}{2}\right)^{2}}{\sigma_{b}^{2}}\right] \cos \left(2 x^{\prime} D_{p}-d_{x} D_{p}\right) d x^{\prime} \\
& +\exp \left[-\frac{d_{x}^{2}}{2 \sigma_{b}^{2}}\right] \exp \left[\frac{V^{2}}{4 \sigma_{b}^{2}}\right] \cos \left(-d_{x} D_{p}\right) \int_{-\infty}^{\infty} \exp \left[-\frac{\left(x^{\prime}+\frac{V}{2}\right)^{2}}{\sigma_{b}^{2}}\right] d x^{\prime}
\end{aligned}
$$

By using the variables transformation,

$$
x^{\prime}=x+\frac{V}{2}
$$

We obtain the first term in Eq. (D.12) as

$$
\propto \exp \left[-\frac{d_{x}^{2}}{2 \sigma_{b}^{2}}\right] \exp \left[\frac{V^{2}}{4 \sigma_{b}^{2}}\right] \int_{-\infty}^{\infty} \exp \left[-\frac{x^{2}}{\sigma_{b}^{2}}\right] \cos \left(2 x D_{p}-\left(V+d_{x}\right) D_{p}\right) d x
$$

By applying the following trigonometry identities

$$
\begin{aligned}
\cos \left(2 x D_{p}-\left(V+d_{x}\right) D_{p}\right)= & \cos \left(2 x D_{p}\right) \cos \left(-\left(V+d_{x}\right) D_{p}\right)+\sin \left(2 x D_{p}\right) \sin \left(-\left(V+d_{x}\right) D_{p}\right) \\
& \cos \left(-\left(V+d_{x}\right) D_{p}\right)=\cos \left(\left(V+d_{x}\right) D_{p}\right)
\end{aligned}
$$

We can rewrite Eq. (D.13) as

$$
\propto \exp \left[-\frac{d_{x}^{2}}{2 \sigma_{b}^{2}}\right] \exp \left[\frac{V^{2}}{4 \sigma_{b}^{2}}\right] \cos \left(\left(V+d_{x}\right) D_{p}\right) \int_{-\infty}^{\infty} \exp \left[-\frac{x^{2}}{\sigma_{b}^{2}}\right] \cos \left(2 x D_{p}\right) d x
$$


By integrating over $\mathrm{x}$ and using $V+d_{x}=i \frac{\mathrm{k} d_{p} \sigma_{b}^{2}}{f}$, we obtain Eq. (D.16) as

$$
\begin{array}{r}
\propto \exp \left[-\frac{d_{x}^{2}}{2 \sigma_{b}^{2}}\right] \exp \left[\frac{V^{2}}{4 \sigma_{b}^{2}}\right] \exp \left[-D_{p}{ }^{2} \sigma_{b}^{2}\right] \cos \left(i \frac{\mathrm{k} d_{p} \sigma_{b}^{2}}{f} D_{p}\right) . \\
\propto \exp \left[-\frac{d_{x}^{2}}{2 \sigma_{b}^{2}}\right] \exp \left[\frac{\left(-d_{x}+i \frac{\mathrm{k} d_{p} \sigma_{b}^{2}}{f}\right)^{2}}{4 \sigma_{b}^{2}}\right] \exp \left[-D_{p}^{2} \sigma_{b}^{2}\right]\left(\exp \left[\frac{\mathrm{k} d_{p} \sigma_{b}^{2}}{f} D_{p}\right]+\exp \left[-\frac{\mathrm{k} d_{p} \sigma_{b}^{2}}{f} D_{p}\right]\right) \cdot(
\end{array}
$$

For the second term in Eq. (D.12), integrating over $x^{\prime}$ we obtain

$$
\propto \sqrt{\sigma_{b}^{2} \pi} \exp \left[-\frac{d_{x}^{2}}{2 \sigma_{b}^{2}}\right] \exp \left[\frac{\left(-d_{x}+i \frac{\mathrm{k} d_{p} \sigma_{b}^{2}}{f}\right)^{2}}{4 \sigma_{b}^{2}}\right] \cos \left(d_{x} D_{p}\right) .
$$

By summation of e Eq. (D.18) and (D.19), $V_{B_{\text {III }}}\left(d_{x}, d_{p}\right)$ is obtained as

$$
\begin{aligned}
& \propto \exp \left[-\frac{d_{x}^{2}}{2 \sigma_{b}^{2}}\right] \exp \left[\frac{V^{2}}{4 \sigma_{b}^{2}}\right] \exp \left[-D_{p}{ }^{2} \sigma_{b}^{2}\right]\left(\exp \left[\frac{\mathrm{k} d_{p} \sigma_{b}^{2}}{f} D_{p}\right]+\exp \left[-\frac{\mathrm{k} d_{p} \sigma_{b}^{2}}{f} D_{p}\right]\right) \\
& +\sqrt{\sigma_{b}^{2} \pi} \exp \left[-\frac{d_{x}^{2}}{2 \sigma_{b}^{2}}\right] \exp \left[\frac{V^{2}}{4 \sigma_{b}^{2}}\right] \cos \left(d_{x} D_{p}\right) .
\end{aligned}
$$

However, the first term in above equation vanishes to zero leaving

$$
V_{B_{\text {III }}}\left(d_{x}, d_{p}\right) \propto \sqrt{\sigma_{b}^{2} \pi} \exp \left[-\frac{d_{x}^{2}}{2 \sigma_{b}^{2}}\right] \exp \left[\frac{V^{2}}{4 \sigma_{b}^{2}}\right] \cos \left(d_{x} D_{p}\right)
$$

Summation of Eq. (D.6), Eq. (D.9) and Eq. (D.21) leads to the interference signal, $V_{B}\left(d_{x}, d_{p}\right)$. Then, we can obtain propensity which is the mean-square interference signal interference signal as

$$
\left|V_{B}\left(d_{x}, d_{p}\right)\right|^{2} \propto\left|V_{B_{\mathrm{I}}}\left(d_{x}, d_{p}\right)+V_{B_{\mathrm{II}}}\left(d_{x}, d_{p}\right)+V_{B_{\mathrm{III}}}\left(d_{x}, d_{p}\right)\right|^{2}
$$




$$
\begin{gathered}
\propto \mid\left\{\exp \left(i \frac{a \mathrm{k} d_{p}}{f}\right)+\exp \left(-i \frac{a \mathrm{k} d_{p}}{f}\right)\right\} \exp \left[-\frac{d_{x}^{2}}{2 \sigma_{a}^{2}}\right] \exp \left[-\frac{B^{2} \sigma_{a}^{2}}{4}\right] \\
+\left.\exp \left[-\frac{d_{x}^{2}}{2 \sigma_{b}^{2}}\right] \exp \left[\frac{V^{2}}{4 \sigma_{b}^{2}}\right] \cos \left(d_{x} D_{p}\right)\right|^{2} .
\end{gathered}
$$

Substituting $B=-d_{x}+i \frac{\mathrm{k} d_{p} \sigma_{a}^{2}}{f}, V=i \frac{\mathrm{k} d_{p} \sigma_{b}^{2}}{f}-d_{x}, \delta_{p}=\frac{\mathrm{k} d_{p}}{f}$ and applying the trigonometry identity $\exp \left(i a \delta_{p}\right)+\exp \left(i a \delta_{p}\right)=\cos \left(a \delta_{p}\right)$, we obtain

$$
\begin{aligned}
\left|V_{B}\left(d_{x}, d_{p}\right)\right|^{2} \propto & \mid \exp \left[\frac{1}{4 \sigma_{b}^{2}}\left(d_{x}^{2}-\delta_{p}^{2} \sigma_{b}^{4}-2 i d_{x} \delta_{p} \sigma_{b}^{2}\right)\right] \cos \left(d_{x} D_{p}\right) \\
& +\left.\exp \left[\frac{1}{4 \sigma_{a}^{2}}\left(d_{x}^{2}-\delta_{p}^{2} \sigma_{a}^{4}-2 i d_{x} \delta_{p} \sigma_{a}^{2}\right)\right] \cos \left(a \delta_{p}\right)\right|^{2} \\
& \sim\left|\cos \left(d_{x} D_{p}\right)+\cos \left(a \delta_{p}\right)\right|^{2}
\end{aligned}
$$




\section{Appendix E}

\section{Variances of Position and Momentum}

The variances of position $(\Delta X)^{2}$ and momentum $(\Delta P)^{2}$ of the EPR correlations can be obtained later through the evaluation of $\mathcal{P}_{b}(X, P)$, which is given by

$$
\begin{aligned}
\mathcal{P}_{b}(X, P) \propto\left|V_{B}(X, P)\right|^{2} \\
\\
\sim\left|\cos \left(X D_{p}\right)+\cos \left(P \delta_{p}\right)\right|^{2} .
\end{aligned}
$$

The variance for position is expressed as

$$
(\Delta X)^{2}=\left\langle X^{2}\right\rangle-\langle X\rangle^{2},
$$

where $\left\langle X^{2}\right\rangle$ is expectation value for $X^{2}$ and $\langle X\rangle$ is expectation value for $X$. Expectation value for $X^{2}$ is given as,

$$
\begin{aligned}
\left\langle X^{2}\right\rangle & =\frac{D_{p}}{2 \pi} \int_{\frac{-\pi}{D p}}^{\frac{\pi}{D p}} X^{2}\left|V_{B}(X)\right|^{2} d X, \\
& =\frac{D_{p}}{2 \pi} \int_{\frac{-\pi}{D p}}^{\frac{\pi}{D p}} X^{2}\left|\cos \left(X D_{p}\right)\right|^{2} d X,
\end{aligned}
$$




$$
\begin{aligned}
& =\frac{D_{p}}{2 \pi}\left\{\frac{1}{2} \int_{\frac{-\pi}{D_{p}}}^{\frac{\pi}{D_{p}}} X^{2} d X+\frac{1}{2} \int_{\frac{-\pi}{D_{p}}}^{\frac{\pi}{D_{p}}} X^{2} \cos \left(2 X D_{p}\right) d X\right\}, \\
& =\frac{D_{p}}{2 \pi}\left\{\frac{\pi^{3}}{3 D_{p}^{3}}+\frac{1}{2} \int_{\frac{-\pi}{D_{p}}}^{\frac{\pi}{D_{p}}} X^{2} \cos \left(2 X D_{p}\right) d X\right\} .
\end{aligned}
$$

Solving the second term using integration by part, leaving

$$
\begin{aligned}
\frac{1}{2} \int_{\frac{-\pi}{D_{p}}}^{\frac{\pi}{D_{p}}} X^{2} \cos \left(2 X D_{p}\right) d X & =\frac{1}{2}\left[\frac{X^{2} \sin 2 X D_{p}}{2 D_{p}}\right]_{\frac{-\pi}{D_{p}}}^{\frac{\pi}{D_{p}}}-\frac{1}{2} \int_{\frac{-\pi}{D_{p}}}^{\frac{\pi}{D_{p}}} \frac{\sin \left(2 X D_{p}\right) 2 X}{2 D_{p}} d X \\
& =0-\frac{1}{2} \int_{\frac{-\pi}{D_{p}}}^{\frac{\pi}{D_{p}}} \frac{\sin \left(2 X D_{p}\right) 2 X}{2 D_{p}} d X
\end{aligned}
$$

Again, solving by integration by parts to obtain

$$
\begin{aligned}
-\frac{1}{2 D_{p}} \int_{\frac{-\pi}{D_{p}}}^{\frac{\pi}{D_{p}}} \sin \left(2 X D_{p}\right) X d X & =-\frac{1}{2 D_{p}}\left\{\left[\frac{-X \cos \left(2 X D_{p}\right)}{2 D_{p}}\right]_{\frac{-\pi}{D_{p}}}^{\frac{\pi}{D_{p}}}+\int_{\frac{-\pi}{D_{p}}}^{\frac{\pi}{D_{p}}}-\frac{\cos \left(2 X D_{p}\right)}{2 D_{p}} d X\right\} \\
& =-\frac{1}{2 D_{p}}\left\{\left[\frac{-X \cos \left(2 X D_{p}\right)}{2 D_{p}}\right]_{\frac{-\pi}{D_{p}}}^{\frac{\pi}{D_{p}}}+\frac{1}{2 D_{p}}\left[\frac{\sin 2 X D_{p}}{2 D_{p}}\right]_{\frac{-\pi}{D_{p}}}^{\frac{\pi}{D_{p}}}\right\} \\
& =\frac{1}{2 D_{p}}\left\{\frac{\pi}{D_{p}{ }^{2}}\right\}
\end{aligned}
$$

Finally, we obtain

$$
\left\langle X^{2}\right\rangle=\frac{1}{2 \pi}\left(\frac{\pi}{2 D_{p}^{2}}+\frac{\pi^{3}}{3 D_{p}^{2}}\right)
$$

Similar, expectation value for $X$ is given as, 


$$
\begin{gathered}
\langle X\rangle=\frac{D_{p}}{2 \pi} \int_{\frac{-D_{p}}{\pi}}^{\frac{D_{p}}{\pi}} X\left|V_{B}(X)\right|^{2} d X=\frac{D_{p}}{2 \pi} \int_{\frac{-\pi}{D_{p}}}^{\frac{-\pi}{D_{p}}} X\left|\cos \left(X D_{p}\right)\right|^{2} d X \\
=\frac{D_{p}}{2 \pi}\left\{\frac{1}{2} \int_{\frac{-\pi}{D_{p}}}^{\frac{\pi}{D_{p}}} X d X+\frac{1}{2} \int_{\frac{-\pi}{D_{p}}}^{\frac{\pi}{D_{p}}} X \cos \left(2 X D_{p}\right) d X\right\} \\
=\frac{D_{p}}{2 \pi}\left\{0+\frac{1}{2} \int_{\frac{-\pi}{D_{p}}}^{\frac{\pi}{D_{p}}} X \cos \left(2 X D_{p}\right) d X\right\}
\end{gathered}
$$

Solving using integration by parts, we obtain

$$
\begin{aligned}
\frac{1}{2} \frac{D_{p}}{2 \pi} \int_{\frac{-\pi}{D_{p}}}^{\frac{\pi}{D_{p}}} X \cos \left(2 X D_{p}\right) d X & =\frac{1}{2} \frac{D_{p}}{2 \pi}\left\{\left[\frac{-X \sin \left(2 X D_{p}\right)}{2 D_{p}}\right]_{\frac{-\pi}{D_{p}}}^{\frac{\pi}{D_{p}}}-\int_{\frac{-\pi}{D_{p}}}^{\frac{\pi}{D_{p}}} \frac{\cos \left(2 X D_{p}\right)}{2 D_{p}} d X\right\} \\
& =\frac{1}{2} \frac{D_{p}}{2 \pi}\left\{o-\frac{1}{2 D_{p}}\left[\frac{-\sin \left(2 X D_{p}\right)}{2 D_{p}}\right]_{\frac{-\pi}{D_{p}}}^{\frac{\pi}{D_{p}}}\right\}=0 .
\end{aligned}
$$

The variance for position as

$$
\begin{aligned}
(\Delta X)^{2} & =\left\langle X^{2}\right\rangle-\langle X\rangle^{2} \\
& =\frac{1}{2}\left(\frac{1}{2 D_{p}^{2}}+\frac{\pi^{2}}{3 D_{p}^{2}}\right) .
\end{aligned}
$$

In a similar fashion, variance for momentum can be calculated as,

$$
\begin{aligned}
(\Delta P)^{2} & =\left\langle P^{2}\right\rangle-\langle P\rangle^{2}, \\
& =\frac{1}{2}\left(\frac{1}{2 a^{2}}+\frac{\pi^{2}}{3 a^{2}}\right) .
\end{aligned}
$$


Appendix F

Properties of Highly Non-linear fiber
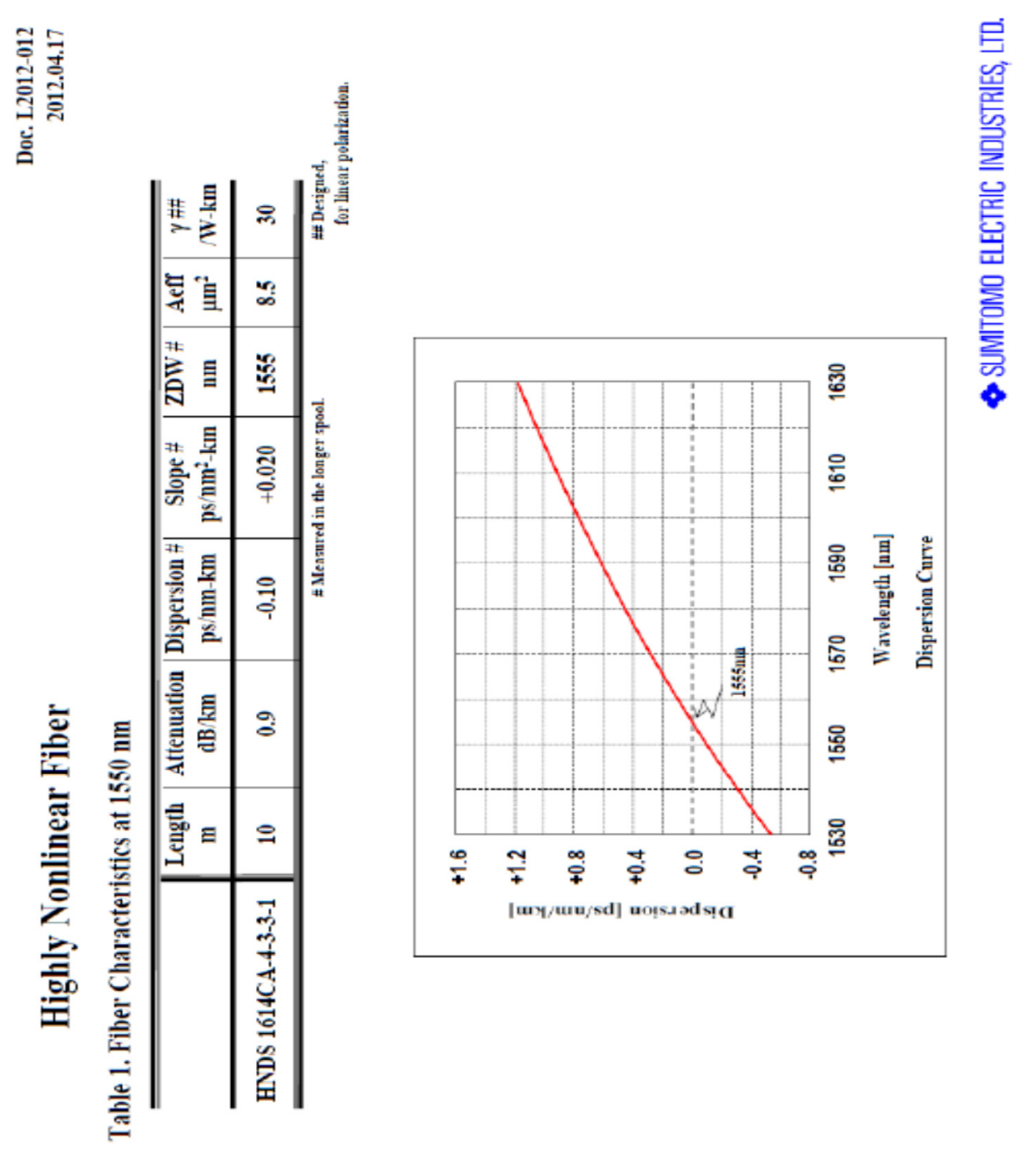


\section{Appendix G}

\section{Copyright Permissions:}

1. Copyright permission from the American Physical Society (APS) to reproduce texts, figures and data published in Chapter 2 and Chapter 3 of this dissertation.

Dinchifgamises

Yong Meng Sua <ysua@mtu.edu>

\section{Re: Request to reproduce article}

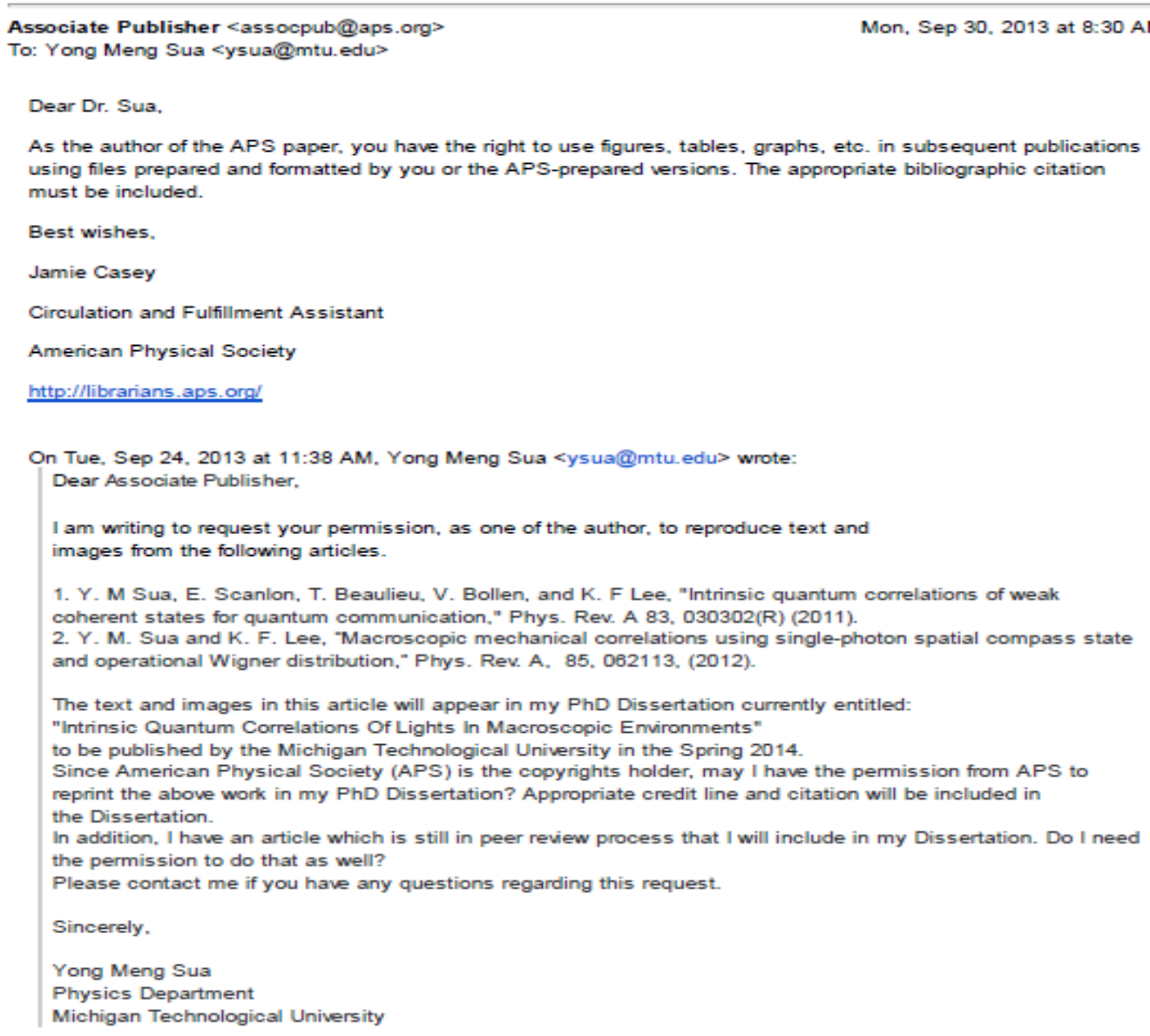


2. Copyright permission from the Intech to reproduce published texts, figures and data in Chapter 3 of this dissertation.

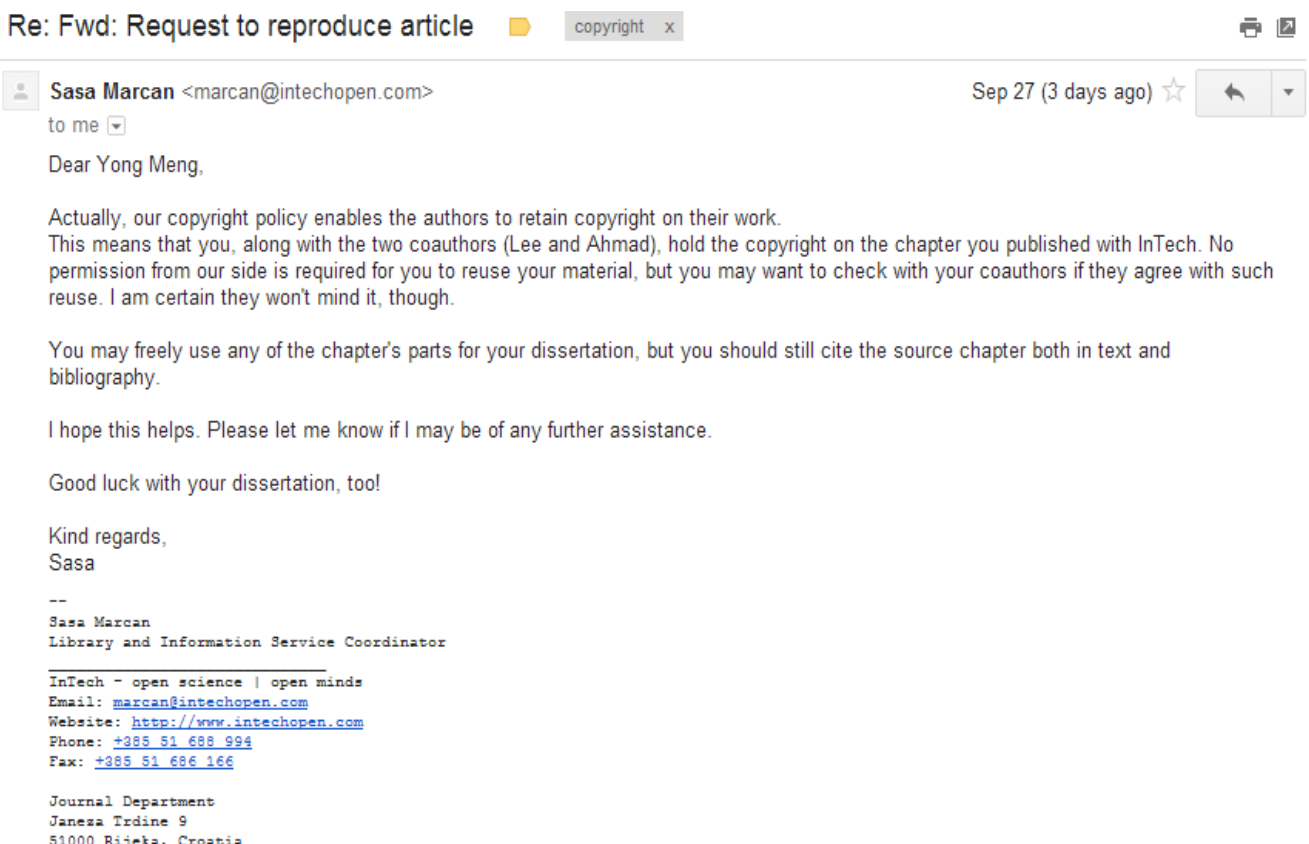

3. Copyright permission from the Optical Society of America (OSA) to reproduce texts, figures and data published in Chapter 4 of this dissertation.

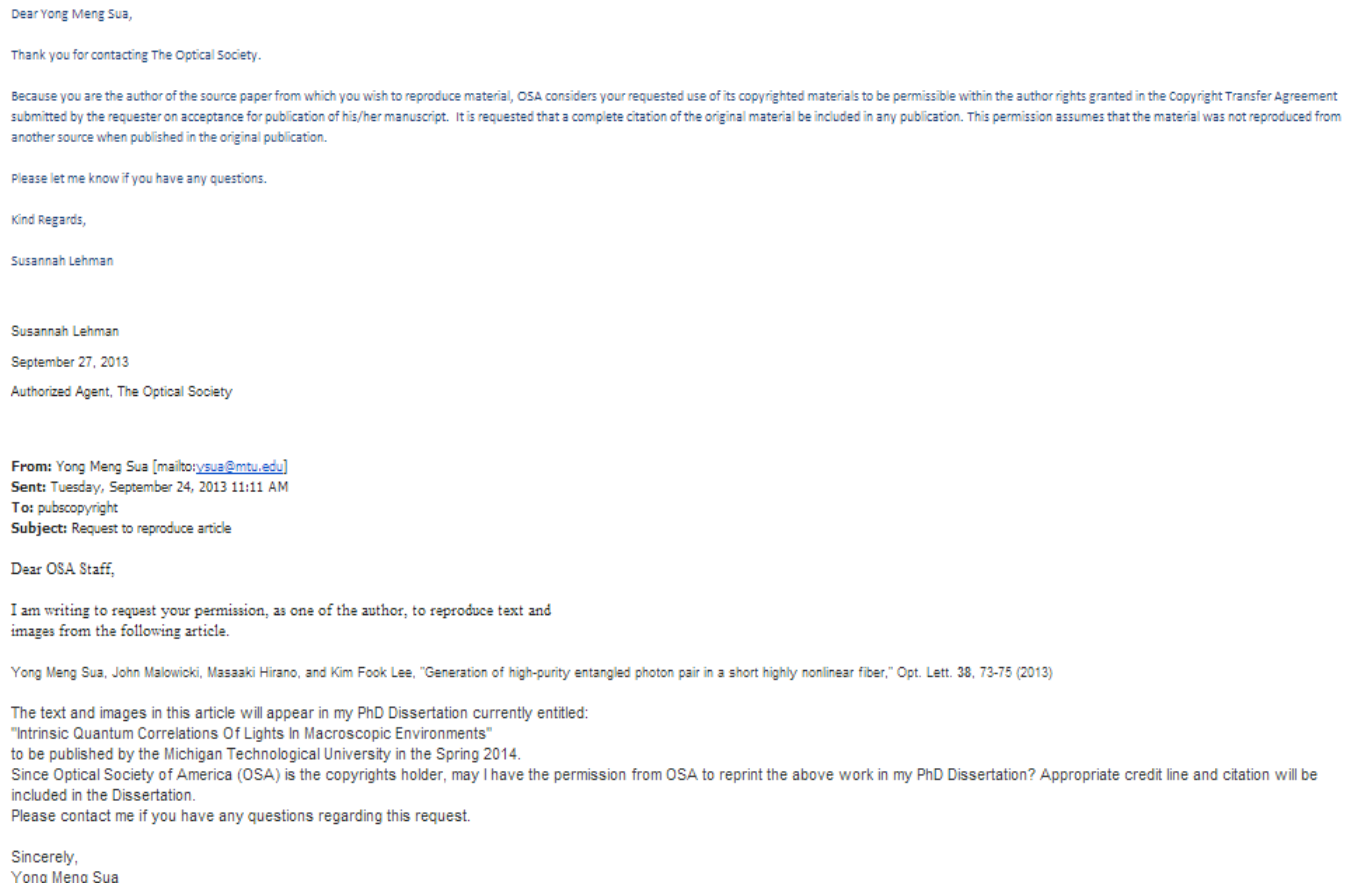


4. Copyright permission from the Optical Society of America (OSA) to reproduce texts, figures and data published in Chapter 5 of this dissertation.

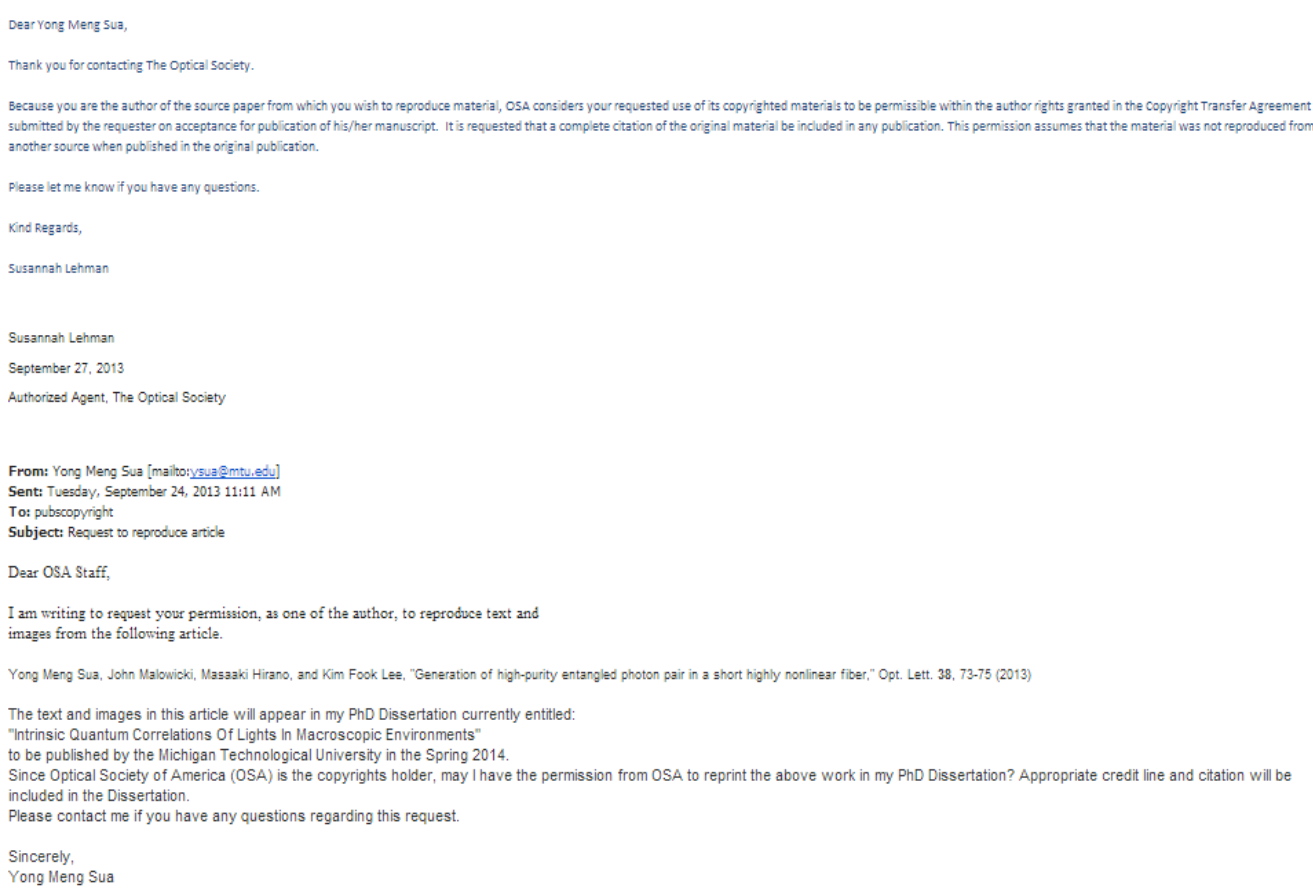

